\title{
WestVirginiaUniversity
}

THE RESEARCH REPOSITORY @ WVU

Graduate Theses, Dissertations, and Problem Reports

2005

\section{Nondestructive evaluation of wooden logs using ground penetrating radar}

\author{
Sachin Agrawal \\ West Virginia University
}

Follow this and additional works at: https://researchrepository.wvu.edu/etd

\section{Recommended Citation}

Agrawal, Sachin, "Nondestructive evaluation of wooden logs using ground penetrating radar" (2005). Graduate Theses, Dissertations, and Problem Reports. 1619.

https://researchrepository.wvu.edu/etd/1619

This Thesis is protected by copyright and/or related rights. It has been brought to you by the The Research Repository @ WVU with permission from the rights-holder(s). You are free to use this Thesis in any way that is permitted by the copyright and related rights legislation that applies to your use. For other uses you must obtain permission from the rights-holder(s) directly, unless additional rights are indicated by a Creative Commons license in the record and/ or on the work itself. This Thesis has been accepted for inclusion in WVU Graduate Theses, Dissertations, and Problem Reports collection by an authorized administrator of The Research Repository @ WVU. For more information, please contact researchrepository@mail.wvu.edu. 


\title{
NONDESTRUCTIVE EVALUATION OF WOODEN LOGS USING GROUND PENETRATING RADAR
}

\author{
Sachin Agrawal
}

\author{
Thesis submitted to the \\ College of Engineering and Mineral Resources \\ at West Virginia University \\ in partial fulfillment of the requirements \\ for the degree of
}
Master of Science
in
Civil Engineering

Udaya B. Halabe, Ph.D., P.E., Chair

Hema J. Siriwardane, Ph.D., P.E.

Bhaskaran Gopalakrishnan, Ph.D., P.E.

Department of Civil and Environmental Engineering

\author{
Morgantown, West Virginia \\ 2005
}

Keywords: Ground penetrating radar, wood, logs, subsurface defects, knots, decays, rots, embedded metals, dielectric constant 


\section{ABSTRACT}

\section{NONDESTRUCTIVE EVALUATION OF WOODEN LOGS USING GROUND PENETRATING RADAR}

\section{Sachin Agrawal}

Presently there are no suitable non-invasive methods for precisely detecting the subsurface defects in logs in real time. Internal defects such as knots, decays, and embedded metals are of greatest concern for lumber production. While defects such as knots and decays (rots) are of major concern related to productivity and yield of high value wood products, embedded metals can damage the saw blade and significantly increase the down time and maintenance costs of saw mills.

Nondestructive scanning of logs using techniques such as Ground Penetrating Radar (GPR) prior to sawing can greatly increase the productivity and yield of high value lumber. Currently GPR has been widely used for civil engineering applications for nondestructive testing of bridges and highways. GPR is also being used for geological and archeological purposes where the imaging needs in many ways are similar to that of logs. However, GPR application in the field of wood industry is very new. GPR has advantages both technically and economically over other non-invasive techniques like Xray, Nuclear Magnetic Resonance (NMR), Stress Wave and Ultrasound. GPR can collect subsurface data much faster compared to many other techniques and provides better penetrating power. For every application the frequency of EM wave plays a very important role.

The main objective of this research is to investigate the use GPR for identifying subsurface defects in logs. Subsurface defects cause major problems to sawmills and veneer mills. Studies have demonstrated a gain of up to $21 \%$ by sawing logs under different orientations and using different sawing patterns (Gupta et al. 1998). This is possible only if the defects can be located accurately with mapping of their spatial extent. Such spatial mapping of subsurface defects can be combined with computer analysis to determine the optimal sawing pattern.

The major defects which are of concern are knots, decays or rots, and embedded metal objects. The current technique using metal detectors cannot pinpoint the exact location of embedded metals in logs and also are not capable of detecting knots and rots. Techniques using stress wave or ultrasound can detect knots and rots to an extent, but are too slow in gathering data, thus making them totally unsuitable for real-time on-line implementation in saw mills.

On the other hand, the GPR technique allows rapid data collection capability. Therefore, this research investigated the capability of GPR in determining the position and type of subsurface defects in logs. The depth resolution of defects using GPR was found to be within $0.04 \mathrm{~m}$ and the position along the length of the log could be predicted within $0.02 \mathrm{~m}$. Based on the results, it was concluded that GPR can detect subsurface defects such as metals, knots, and decays (rots) and is a very promising techniques for future on-line implementation in saw mills. 


\section{ACKNOWLEDGEMENTS}

I am thankful to my AEC Committee Chair and my research advisor, Dr. Udaya B. Halabe, for his valuable guidance and encouragement throughout the period of my Masters of Science in Civil Engineering (M.S.C.E) study and research at WVU. I am sincerely thankful for his patience and time when guiding me. I am thankful for the valuable suggestions provided by Dr. Bhaskaran Gopalakrishnan and Dr. Hema J. Siriwardane, members of my Advisory and Examining Committee.

I extend my thanks to Dr. Joe McNeel, Director of Division of Forestry for all the encouragement provided during my research. I wish to specially thank Mr. Shawn Grushecky, Assistant Director of WVU's Appalachian Hardwood Center, for providing valuable laboratory assistance in sawing the logs used in this research.

I am thankful to Jerry Nestor and Bill Comstock for their help in the laboratory. I am thankful to my colleagues, Mr. Cheng Lok Hing and Mr. Shasanka Shekhar Dutta, for all their help during my stay at WVU. I would also like to thank my roommates for making my stay in Morgantown a very pleasant and memorable one.

I am thankful to my family, who made me realize the importance of advanced studies. Their support and love gave me the motivation to do my best in life.

I would like to acknowledge the funding provided by the U. S. Department of Agriculture for this research under Grant/Contract No. 2004-34158-14390. 


\section{Table of Contents}

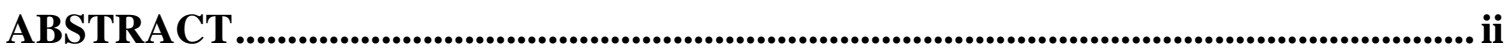

ACKNOWLEDGEMENTS .................................................................................................iii

Table of Contents ................................................................................................................. iv

List of Figures......................................................................................................... vi

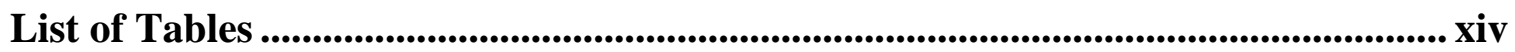

Chapter 1 INTRODUCTION.................................................................................................. 1

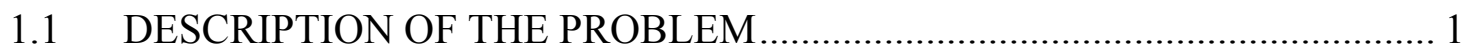

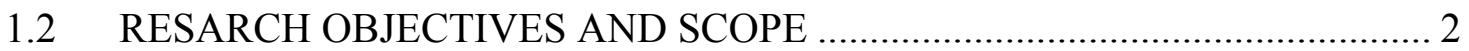

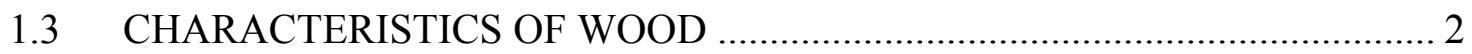

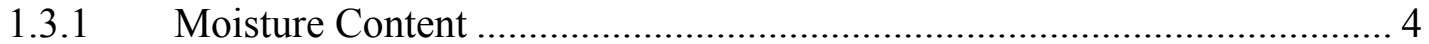

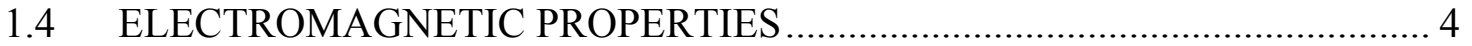

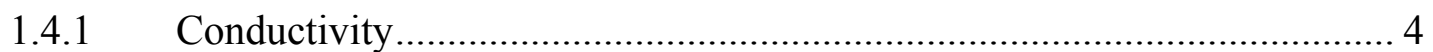

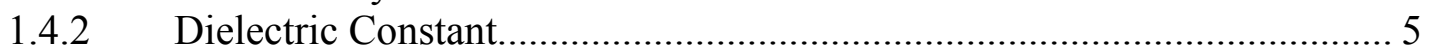

1.4.3 Complex Dielectric Permittivity …………........................................... 5

1.5 NATURAL CHARACTERISTICS AFFECTING MECHANICAL

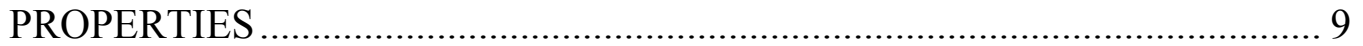

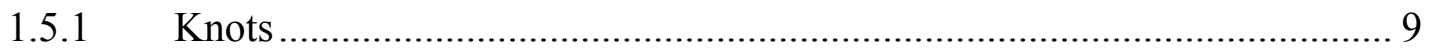

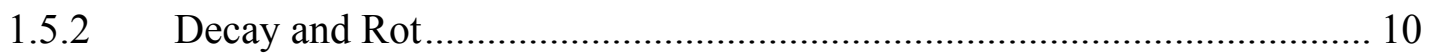

1.5.3 Embedded Metals........................................................................... 11

1.6 ORGANIZATION OF THE THESIS..................................................... 11

Chapter 2 GPR FUNDAMENTALS ................................................................................ 12

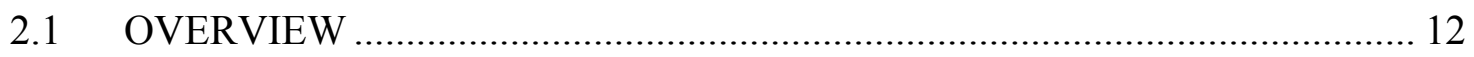

2.2 ELECTROMAGNETIC WAVES ……….............................................. 15

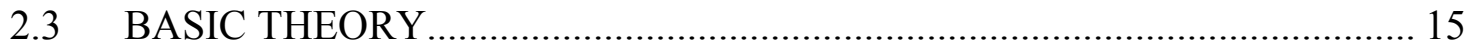

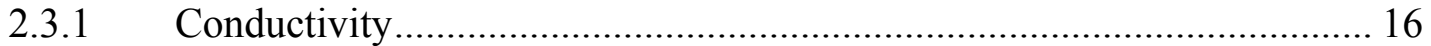

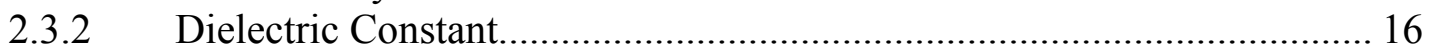

2.4 ATTENUATION OF ELECTROMAGNETIC WAVES..................................... 17

2.5 REFLECTION AND TRANSMISSION OF RADAR WAVES AT AN

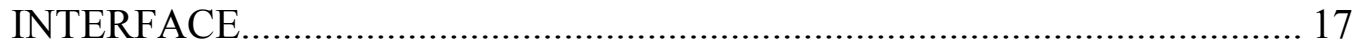

2.6 EFFECTIVE RANGE AND RESOLUTION .............................................. 18

2.7 EFFECT OF DEFECTS ON RECEIVED SIGNALS …………..................... 19

2.8 GPR AS A PREFERED TECHNIQUE ..................................................... 19

2.9 LITERATURE REVIEW ON USE OF GPR FOR WOOD INSPECTION...... 20

Chapter 3 EXPERIMENTAL SETUP ........................................................................... 22

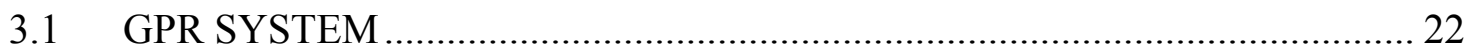

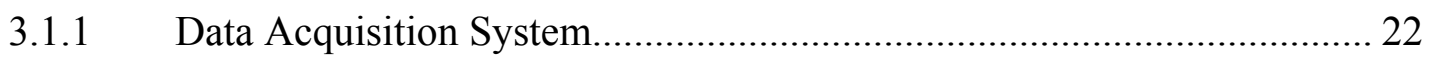

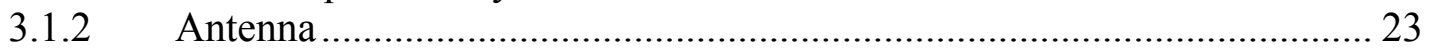


3.1.3 Antenna Deployment Frame ………………...................................... 24

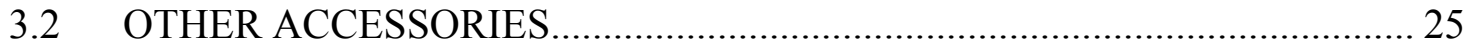

Chapter 4 DATA ACQUISITION AND INTERPRETATION PROCEDURE ..... 30

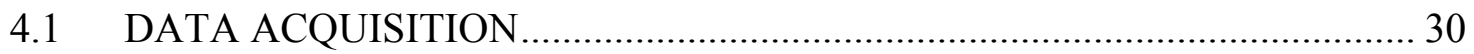

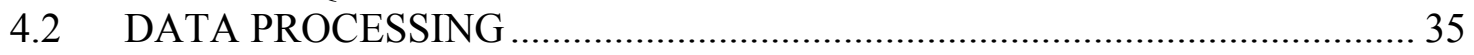

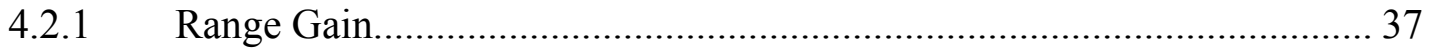

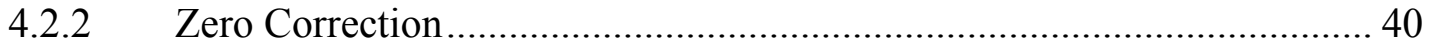

4.3 MEASUREMENT OF DIELECTRIC CONSTANT ..................................... 48

Chapter 5 EXPERIMENTAL RESULTS........................................................................ 50

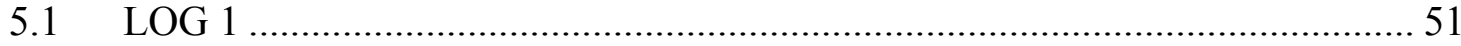

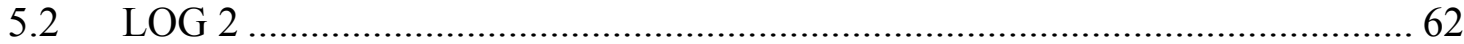

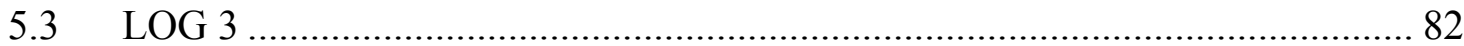

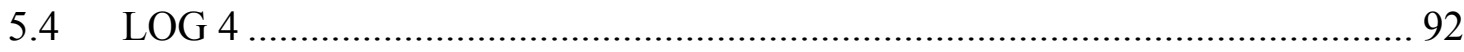

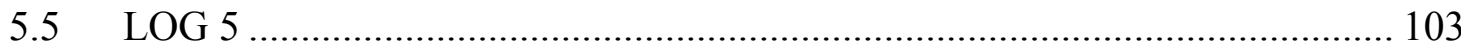

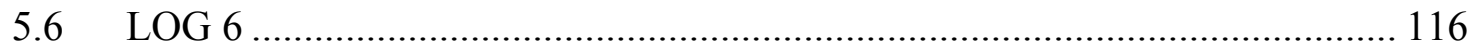

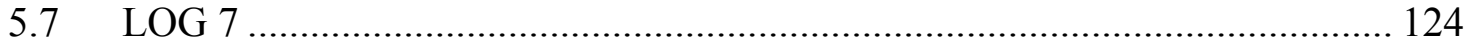

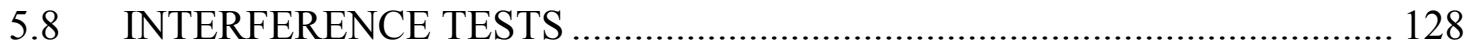

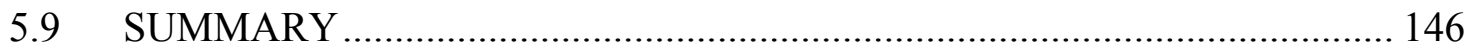

Chapter 6 CONCLUSIONS AND RECOMMENDATIONS...................................... 148

REFERENCES.................................................................................................................. 150 


\section{List of Figures}

Figure 1-1 Cross section of a tree: (a) white oak tree trunk, (b) ponderosa pine log (Miller 1999b) 3

Figure 1-2 Principal axes with respect to grain direction and growth rings (Green et al. 1999)

Figure 1-3 Change in electrical resistance with change in moisture content (Simpson and TenWolde 1999)

Figure 1-4 Dielectric constant as a function of moisture content measured at $2.45 \mathrm{GHz}$ for tangential (T), longitudinal (L) and radial (R) directions for Tamarack (Afzal et al. 2003)

Figure 1-5 Dielectric constant as a function of frequency for tangential (T), longitudinal

(L) and radial (R) direction for Tamarack (Afzal et al. 2003)....

Figure 1-6 Loss tangent as a function of frequency for tangential (T), longitudinal (L) and radial (R) direction for Tamarack (Afzal et al. 2003) .................................... 8

Figure 1-7 Loss tangent as a function of moisture content for tangential (T), longitudinal (L) and radial (R) direction for Tamarack (Afzal et al. 2003). 8

Figure 1-8 Dielectric Constant as a function of moisture content in the transverse direction for different species at frequency $2.38 \mathrm{GHz}$ (Keam Holdem Associates Ltd. 1999)

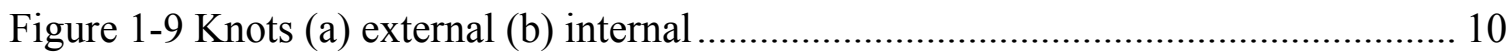

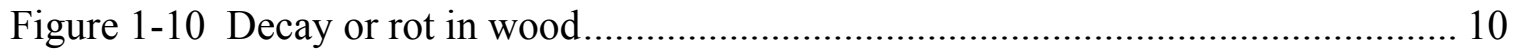

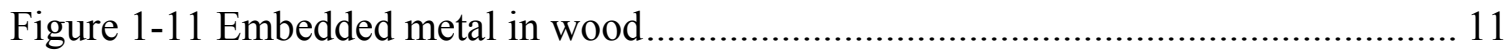

Figure 2-1 Single scan (extracted from sample of scans) with vertical axis showing the strength of the received signal in millivolts $(\mathrm{mV})$ and horizontal axis indicating the two way travel time within the material in nanoseconds (ns).. 13

Figure 2-2 (a) Wiggle plot, (b) grey scale scan, and (c) color scan, of a wooden log, with vertical scale indicating the two-way travel time along the depth of the log in nanoseconds and horizontal scale indicating the travel distance or antenna position along the length of the log in meters....

Figure 3-1 General setup of the GPR data acquisition system ................................... 22

Figure 3-2 Data acquisition system (laptop computer and main frame)........................ 23 


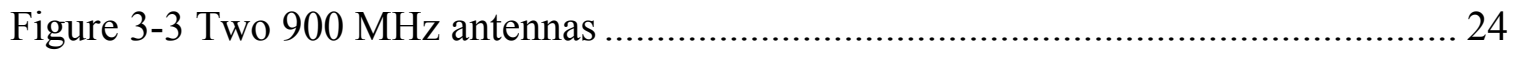

Figure 3-4 Antenna deployment frame .................................................................. 26

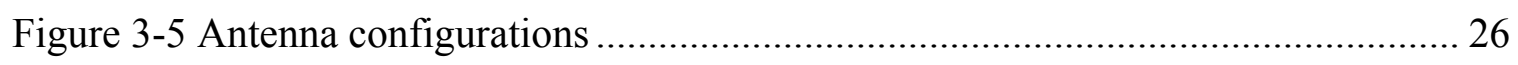

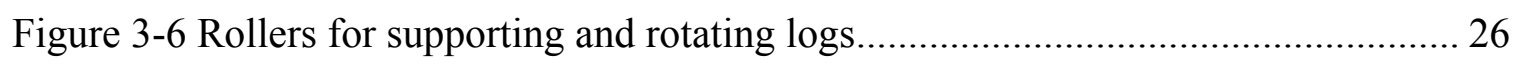

Figure 3-7 (a) Axe, aluminum foils, gloves, measuring tape, and markers, (b) an 18 in saw being used for cutting logs to check for defects ...................................... 27

Figure 3-8 Showing the crane and the control switches for lifting the logs .................... 27

Figure 4-1 Lines marked on $\log 3$ for GPR scanning and sawing purpose ..................... 31

Figure 4-2 Picture showing wooden pieces under the small end of the $\log 4$ to raise the

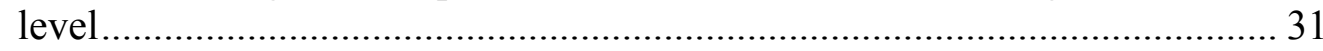

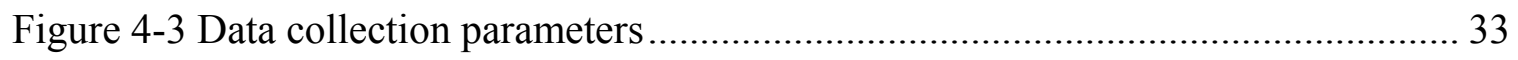

Figure 4-4 Antenna configuration (a) antenna on top (b) antenna on side ....................... 34

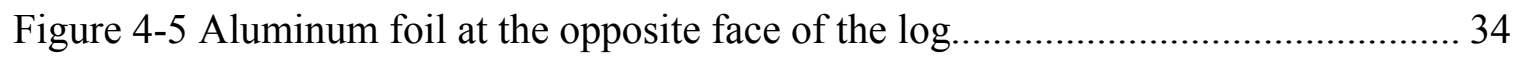

Figure 4-6 Raw GPR data with vertical scale indicating (a) time (ns) (b) depth (m)

(c) sampling points, and the horizontal scale indicating the distance (m) along

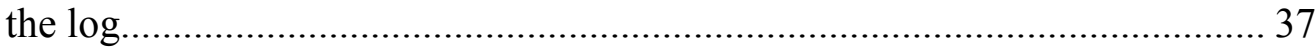

Figure 4-7 Typical radar signal observed using GPR oscilloscope mode ........................ 38

Figure 4-8 Signal comparison for different types of applied gains ................................. 38

Figure 4-9 Typical GPR file of a $\log$ (a) distance vs. time (ns) (b) distance vs. depth (m)

Figure 4-10 GPR data after applying zero correction (a) time (ns) plot (b) depth (m) plot

Figure 4-11 A typical radar signal (viewed in oscilloscope mode) after applying zero correction with the horizontal scale showing (a) two-way travel time (ns) (b) depth (m)

Figure 4-12 FIR filter parameters for Background removal and vertical Low and High

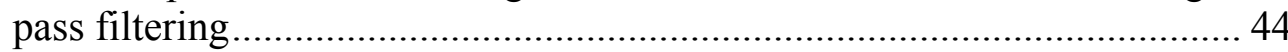

Figure 4-13 GPR data after applying background removal and high pass and low pass filters (a) time (ns) plot (b) depth (m) plot. 46 
Figure 4-14 A typical radar signal (observed in oscilloscope mode) after applying background removal and FIR filtering.................................................. 47

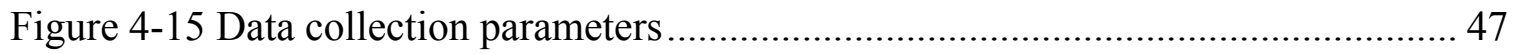

Figure 4-16 GPR signal in RADAN oscilloscope mode used for measuring the dielectric

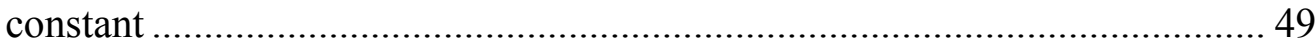

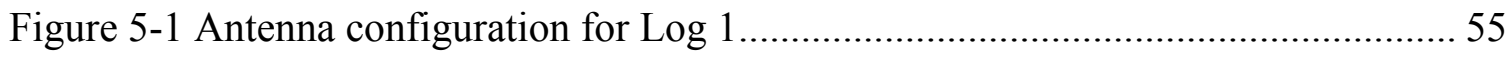

Figure 5-2 (a) Data collection parameters (b) data processing parameters .................... 56

Figure 5-3 Raw GPR data of Log 1, horizontal scale indicating the travel distance along the length of the $\log (\mathrm{m})$ and the vertical scale indicating (a) time (ns) (b) depth (m)

Figure 5-4 Processed GPR data of Log 1, horizontal scale indicating the travel distance along the length of the $\log (\mathrm{m})$ and the vertical scale indicating (a) time (ns) (b) depth (m) 58

Figure 5-5 Processed GPR data of Log 1 indicating the internal features of $\log 1 \ldots \ldots . .59$

Figure 5-6 Cut section profile of Log 1 indicating presence of defects in Log 1 59

Figure 5-7 : Wiggle plot of $\log 1$, horizontal scale indicating travel distance along the length of the $\log (\mathrm{m})$ and vertical scale indicating the depth (m), for (a) raw data, and (b) processed data

Figure 5-8 Comparison of GPR waveforms from sections with and without defects (knots) in Log 1 for (a) raw data (Amplitude vs. Time), (b) processed data (Amplitude vs. Time), and (c) processed data (Amplitude vs. Depth).....

Figure 5-9 Pictures showing (a) Log 2, (b) marks indicating various GPR scanning positions around the $\log$, and (c) antenna position

Figure 5-10 : (a) Data collection parameters (b) data processing parameters

Figure 5-11 Raw GPR data of Log 2 along mark 4, horizontal scale indicating the travel distance along the length of the $\log (\mathrm{m})$ and the vertical scale indicating (a) time (ns) (b) depth (m)

Figure 5-12 Processed GPR data of Log 2 along mark 4, horizontal scale indicating the travel distance along the length of the $\log (\mathrm{m})$ and the vertical scale indicating (a) time (ns) (b) depth (m)

Figure 5-13 Wiggle plot of Log 2 along mark 4, horizontal scale indicating travel distance along the length of the $\log (\mathrm{m})$ and vertical scale indicating the depth (m), for (a) raw data, and (b) processed data 
Figure 5-14 : Raw GPR data of Log 2 along mark 5, horizontal scale indicating the travel distance along the length of the $\log (\mathrm{m})$ and the vertical scale indicating (a) time (ns) (b) depth (m) 73

Figure 5-15 Processed GPR data of Log 2 along mark 5, horizontal scale indicating the travel distance along the length of the $\log (\mathrm{m})$ and the vertical scale indicating (a) time (ns) (b) depth (m)

Figure 5-16 Wiggle plot of Log 2 along mark 5, horizontal scale indicating travel distance along the length of the $\log (\mathrm{m})$ and vertical scale indicating the depth (m), for (a) raw data, and (b) processed data 75

Figure 5-17 Processed GPR data of Log 2 along mark 2, horizontal scale indicating the travel distance along the length of the $\log (\mathrm{m})$ and the vertical scale indicating depth $(\mathrm{m})$ 76

Figure 5-18 Processed GPR data of Log 2 along mark 8, horizontal scale indicating the travel distance along the length of the $\log (\mathrm{m})$ and the vertical scale

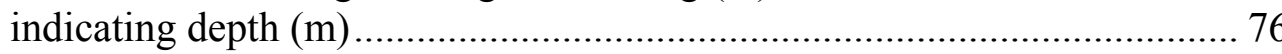

Figure 5-19 Sawing of Log 2 using a portable saw mill ......................................... 77

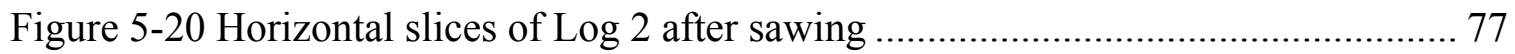

Figure 5-21 Processed data of Figure 5.12 and corresponding defects (knots) ............... 77

Figure 5-22 Processed data of Figure 5.15 and corresponding defects ........................ 78

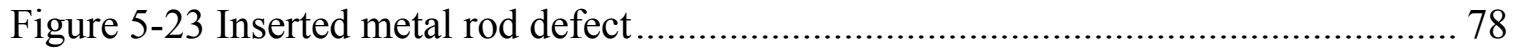

Figure 5-24 Comparison of GPR waveforms along mark 4 from sections with and without defects (knots) in Log 2 for (a) raw data (Amplitude vs. Time), (b) processed data (Amplitude vs. Time), and (c) processed data (Amplitude vs.

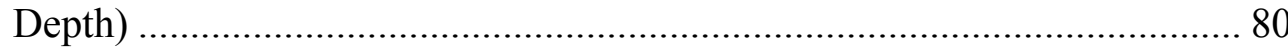

Figure 5-25 Comparison of GPR waveforms along mark 5 from sections with and without defects (knot and metal) in Log 2 for (a) raw data (Amplitude vs. Time), (b) processed data (Amplitude vs. Time), and (c) processed data (Amplitude vs. Depth) .................................................................. 81

Figure 5-26 Pictures showing (a) Log 3 (b) the markings and the decay ....................... 84

Figure 5-27 : Pictures showing (a) metal rod (b) the extent to which the metal could penetrate. 84

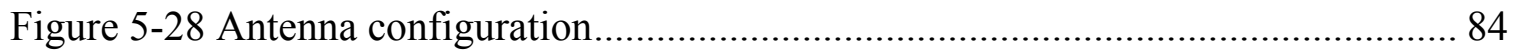

Figure 5-29 (a) Data collection parameters (b) data processing parameters ................... 85 
Figure 5-30 Raw GPR data of $\log 3$, horizontal scale indicating the travel distance along the length of the $\log (\mathrm{m})$ and the vertical scale indicating (a) time (ns) (b) depth (m) 86

Figure 5-31 Processed GPR data of $\log 3$, horizontal scale indicating the travel distance along the length of the $\log (\mathrm{m})$ and the vertical scale indicating (a) time (ns) (b) depth (m) 87

Figure 5-32 Wiggle plot of $\log 3$, horizontal scale indicating travel distance along the length of the $\log (\mathrm{m})$ and vertical scale indicating the depth (m), for (a) raw data, and (b) processed data ........................................................... 88

Figure 5-33 Processed data and corresponding defects in Log 3.............................. 89

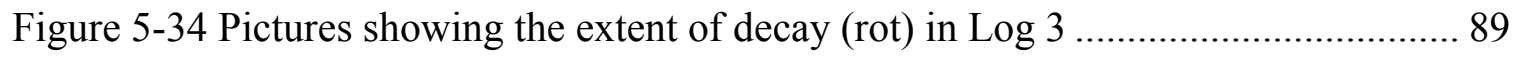

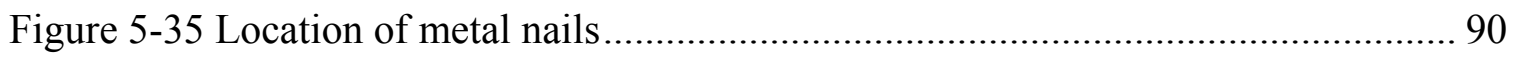

Figure 5-36 Rotten piece and metal nails extracted out from Log 3 ........................... 90

Figure 5-37 Comparison of GPR waveforms from sections with defects (rot and metal) in Log 3 for (a) raw data (Amplitude vs. Time), (b) processed data (Amplitude vs. Time), and (c) processed data (Amplitude vs. Depth) ........................ 92

Figure 5-38 Picture of Log 4 showing the locations where metal rods were inserted...... 95

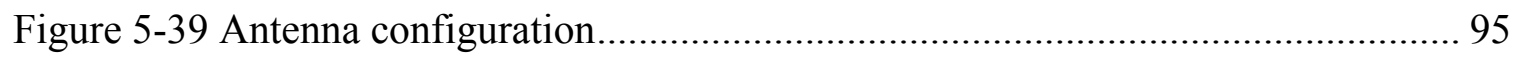

Figure 5-40 (a) Data collection parameters, (b) data processing parameters ................. 96

Figure 5-41 Raw GPR data of Log 4, horizontal scale indicating the travel distance along the length of the $\log (\mathrm{m})$ and the vertical scale indicating (a) time (ns) (b)

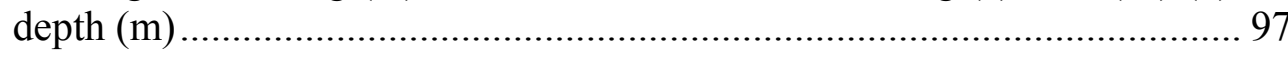

Figure 5-42 Processed GPR data of $\log 4$, horizontal scale indicating the travel distance along the length of the $\log (\mathrm{m})$ and the vertical scale indicating (a) time (ns)

(b) depth (m) 98

Figure 5-43 Wiggle plot of $\log 4$, horizontal scale indicating travel distance along the length of the $\log (\mathrm{m})$ and vertical scale indicating the depth (m), for (a) raw data, and (b) processed data ............................................................... 99

Figure 5-44 Processed GPR data and corresponding locations where metal rods were inserted.... 100

Figure 5-45 Comparison of GPR waveforms from sections with and without defects (metal rods) in Log 4 for (a) raw data (Amplitude vs. Time), (b) processed 
Figure 5-46 Log 5 and the antenna configuration used for collecting GPR data 105

Figure 5-47 Picture showing butt end of Log 5 106

Figure 5-48 (a) Data collection parameters (b) data processing parameters 107

Figure 5-49 Raw GPR data of $\log 5$ (scan \# 5), horizontal scale indicating the travel distance along the length of the $\log (\mathrm{m})$ and the vertical scale indicating (a) time (ns) (b) depth (m)

Figure 5-50 Processed GPR data of $\log 5$ (san \# 5), horizontal scale indicating the travel distance along the length of the $\log (\mathrm{m})$ and the vertical scale indicating (a) time (ns) (b) depth (m)

Figure 5-51 Wiggle plot of Log 5 (scan \# 5), horizontal scale indicating travel distance along the length of the $\log (\mathrm{m})$ and vertical scale indicating the depth (m), for (a) raw data, and (b) processed data. 110

Figure 5-52 Processed data of Log 5 for all six scans, with the predicted internal features marked for mapping subsurface defects

Figure 5-53 Metal defect.

Figure 5-54 Picture showing the problems faced after the saw blade hit the small metal piece and got bent, and subsequent replacement of the blade 112

Figure 5-55 Knots in Log 5.

Figure 5-56 Correlation of processed data and defects after sawing the $\log$.

Figure 5-57 Comparison of GPR waveforms from sections with and without defects (knots and metal) in Log 5 for (a) raw data (Amplitude vs. Time), (b) processed data (Amplitude vs. Time), and (c) processed data (Amplitude vs. Depth) 115

Figure 5-58 Picture of (a) $\log 6$, and (b) rot (cavity) seen clearly at the butt end 118

Figure 5-59 Antenna configuration for $\log 6$ 118

Figure 5-60 (a) Data collection parameters (b) processing parameters 119

Figure 5-61 Raw GPR data of $\log$ 6, horizontal scale indicating the travel distance along the length of the $\log (\mathrm{m})$ and the vertical scale indicating (a) time (ns) (b) depth (m) 120 
Figure 5-62 Processed GPR data of $\log 6$, horizontal scale indicating the travel distance along the length of the $\log (\mathrm{m})$ and the vertical scale indicating (a) time (ns) (b) depth (m) 121

Figure 5-63 Wiggle plot of $\log 6$, horizontal scale indicating travel distance along the length of the $\log (\mathrm{m})$ and vertical scale indicating the depth (m), for (a) raw data, and (b) processed data ................................................................... 122

Figure 5-64 Picture showing cut sections of $\log 4$ 123

Figure 5-65 Processed GPR data of Log 6 and corresponding cut sections showing internal features. 123

Figure 5-66 Picture of Log 7 showing inserted metal. 125

Figure 5-67 Raw GPR data of $\log 7$, vertical scale indicating (a) time (ns) (b) depth (m) 126

Figure 5-68 Processed GPR data of Log 7, vertical scale indicating (a) time (ns) (b) depth (m) 127

Figure 5-69 (a) Top antenna configuration and its (b) data collection parameters 131

Figure 5-70 Raw GPR data for the antenna configuration shown in Figure 5.69, horizontal scale indicating the travel distance along the length of the $\log (\mathrm{m})$ and vertical scale indicating (a) time (ns) (b) depth (m). 132

Figure 5-71 Processed GPR data for the antenna configuration shown in Figure 5.69, horizontal scale indicating the travel distance along the length of the $\log (\mathrm{m})$ and vertical scale indicating (a) time (ns) (b) depth (m).

Figure 5-72 Antenna on the top surface collecting data with side antenna at a distance $0.03 \mathrm{~m}$ from the log surface (b) processed data for the antenna configuration shown in (a).

Figure 5-73 Antenna on the top surface collecting data with side antenna at a distance $0.1 \mathrm{~m}$ from the log surface (b) processed data for the antenna configuration shown in (a).

Figure 5-74 Antenna on the top surface collecting data with side antenna at a distance $0.15 \mathrm{~m}$ from the log surface (b) processed data for the antenna configuration shown in (a) 136

Figure 5-75 Antenna on the top surface collecting data with side antenna completely removed (b) processed data for the antenna configuration shown in (a)... 137

Figure 5-76 Antenna on the top completely removed and single antenna on the side collecting data. 
Figure 5-77 Raw GPR data for the antenna configuration shown in Figure 5-76, horizontal scale indicating the travel distance along the length of the $\log (\mathrm{m})$ and vertical scale indicating time (ns)

Figure 5-78 Processed GPR data for the antenna configuration shown in Figure 5-76, horizontal scale indicating the travel distance along the length of the $\log (\mathrm{m})$ and vertical scale indicating.

Figure 5-79 Antenna configuration for two channel data collection 140

Figure 5-80 Raw GPR data for the antenna configuration shown in Figure 5-79, horizontal scale indicating the travel distance along the length of the $\log (\mathrm{m})$ and vertical scale indicating time (ns)

Figure 5-81 Processed GPR data for the antenna configuration shown in Figure 5-79, horizontal scale indicating the travel distance along the length of the $\log (\mathrm{m})$ and vertical scale indicating

Figure 5-82 Two antenna on the side in T1R1 and T2R2 mode. 142

Figure 5-83 Raw GPR data for the antenna configuration shown in Figure 5-82, horizontal scale indicating the travel distance along the length of the $\log (\mathrm{m})$ and vertical scale indicating time (ns)

Figure 5-84: Processed GPR data for the antenna configuration shown in Figure 5-82, horizontal scale indicating the travel distance along the length of the $\log (\mathrm{m})$ and vertical scale indicating.

Figure 5-85 Two antennas on the side in T1R2 and T2R1 mode

Figure 5-86 Raw GPR data for the antenna configuration shown in Figure 5-85, horizontal scale indicating the travel distance along the length of the $\log (\mathrm{m})$ and vertical scale indicating time (ns)

Figure 5-87 : Processed GPR data of the antenna configuration shown in Figure 5-85, horizontal scale indicating the travel distance along the length of the $\log (\mathrm{m})$ and vertical scale indicating. 145 


\section{List of Tables}

Table 3-1 System Specification (Loulizi 2001, GSSI 2005) ………………………....... 28

Table 3-2 Range of antennas manufactured by GSSI and its applications (GSSI 2005).. 29

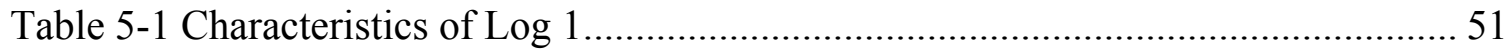

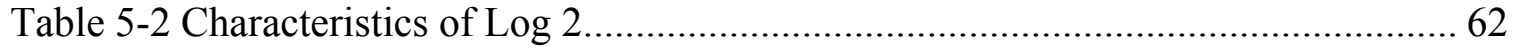

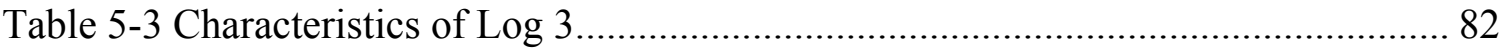

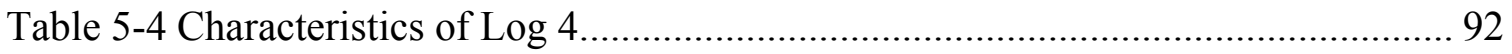

Table 5-5 Comparison between actual and predicted spacing between metals .............. 100

Table 5-6 Comparison between actual and predicted depth for metals ........................... 101

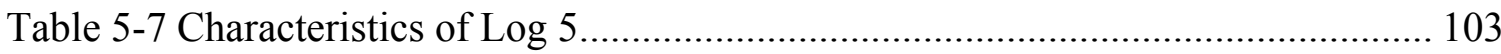

Table 5-8 Comparison between actual and predicted distance and depth for defects .... 106

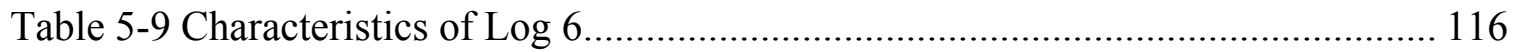

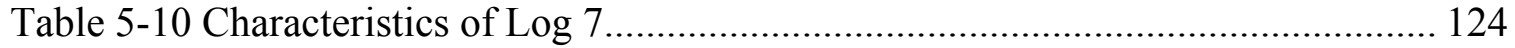

Table 5-11 Summary of detected defects..................................................................... 147

Table 5-12 Summary of metal detectability............................................................... 147 


\section{Chapter 1 \\ INTRODUCTION}

\subsection{DESCRIPTION OF THE PROBLEM}

There has been considerable research in the field of nondestructive testing (NDT) of wood for detecting internal defects, but using elastic waves. The major problem is locating the defects accurately and mapping their spatial extent. Once the defect is accurately located, automated systems could be developed for making the sorting and sawing operation more effective. Precise location of defect combined with computer analysis to determine optimal sawing pattern can increase productivity by $10 \%$ for hardwood and 5 to $8 \%$ for softwood (Forest and Wildlife Research Center, World Wide Web 2005). A study by Gupta et al. (1998) has demonstrated a gain of up to $21 \%$ by sawing logs under different orientations and using different sawing patterns.

The major problem faced by wood processors is the presence of internal defects in logs which are not visible to the naked eyes through surface inspection. The defects can be in the form of knots, decays or rots, and/or presence of embedded metals. If these defects can be located ahead of time, before the log is sawn, significant increase in productivity can be achieved by optimizing the sawing process through active control of the saw blade's orientation. This process can also prevent damage to the saw blade due to embedded metals, thus avoiding downtime and repair costs.

The current elastic wave based technologies such as like ultrasound and stress wave used for detecting metals and defects in wood have the disadvantage that they are time consuming and lack the desired accuracy. Also, scanning technologies like Computed Tomography, X-ray, Ultrasonic and Nuclear Magnetic Resonance have several disadvantages in terms of technical problems and cost involved. The metal detectors currently employed by saw mills can not precisely determine the location of embedded metals, and are useless for defect-detection. Thus, a large number of logs end up being discarded by saw mills, or result in low value wood products since they include defects. 
In order to address the above problem, it is important that a proper scanning technology be used to assess the internal condition of logs before they are sawn. For a factory type setting it is important to implement an on-line scanning technology that can rapidly collect large amount of data and process data accurately in real time.

\subsection{RESARCH OBJECTIVES AND SCOPE}

Ground Penetrating Radar (GPR) can acquire data from logs much more rapidly compared to other NDT methods such as ultrasonics and stress waves techniques. However, the data interpretation is still subjective and needs human intervention. Moreover, the characteristics of GPR signals from wooden logs are not well understood. This research proposes to address the above issues. The objectives of this research are:

- investigate the use of GPR for nondestructive assessment of wooden logs and detect major subsurface defects such as metals, knots, and decays that adversely affect the lumber productivity

- study the characteristics of GPR scans corresponding to the various defect types

- correlate GPR data with actual condition inside the logs observed after sawing the $\operatorname{logs}$

This study evaluates the ability of GPR to detect subsurface defects. The scope includes designing a GPR scanning setup, testing seven logs using GPR and correlating observed anomalies in GPR data with actual information of subsurface defects obtained after sawing the logs. If GPR can detect the defects accurately, then computer algorithms can be written for determining optimal sawing patterns of logs. This would not only increase productivity by increasing the yield of high quality lumber but also reduce the maintenance cost of saw mills by eliminating downtime.

\subsection{CHARACTERISTICS OF WOOD}

Logs can be classified into hardwood and softwood. Generally softwood is used in construction of forms, scaffolding, flooring, poles and piles, and many other building and bridge components. Hardwood is used primarily for architectural, furniture and decorative purposes (Miller 1999a). There are several species of trees in both hardwood and softwood categories, and each species has different characteristics. 
Wood can be characterized as a non-homogeneous, anisotropic material with high degree of variability. The reason for this variability can be explained by taking into account the cross-section of the tree shown in Figure 1-1 (a). The various features shown are (A) outer bark (dry dead tissue), (B) inner bark (living tissue), (C) cambium, (D) sapwood, (E) heartwood, (F) pith, and (G) wood rays (Miller 1999b). The growth ring is clearly shown with a sharp contrast between earlywood and latewood in Figure 1-1 (b).

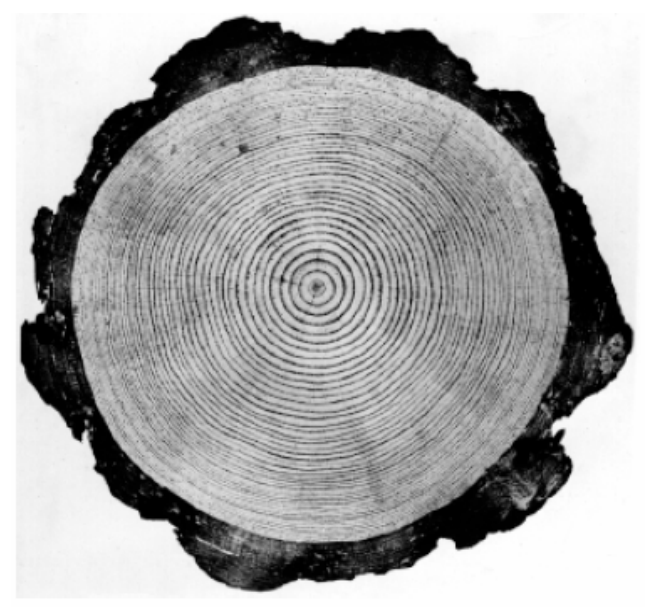

(a)

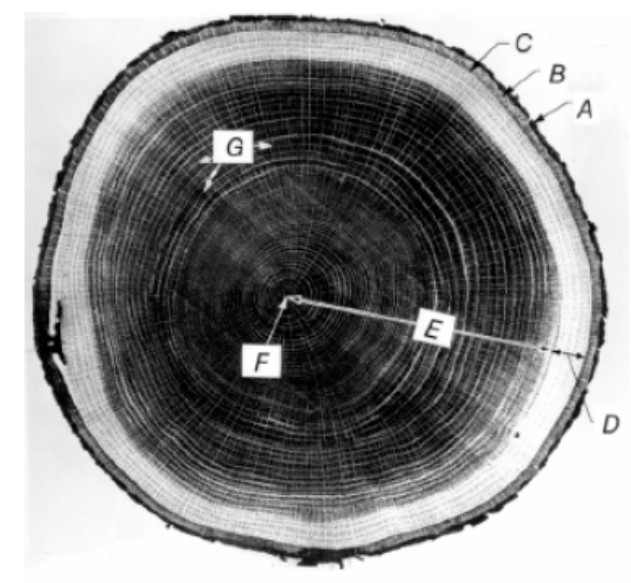

(b)

Figure 1-1 Cross section of a tree: (a) white oak tree trunk, (b) ponderosa pine log (Miller 1999b)

The orthotropic nature of wood makes its electrical and elastic properties unique in three mutually perpendicular axes (Figure 1-2). The axis parallel to fiber is called the longitudinal axis, radial axis is normal to the growth rings and tangential axis is perpendicular to grain but tangential to the growth of the ring (Green et al. 1999). The fibrous nature of wood influences how it is going to be used. 


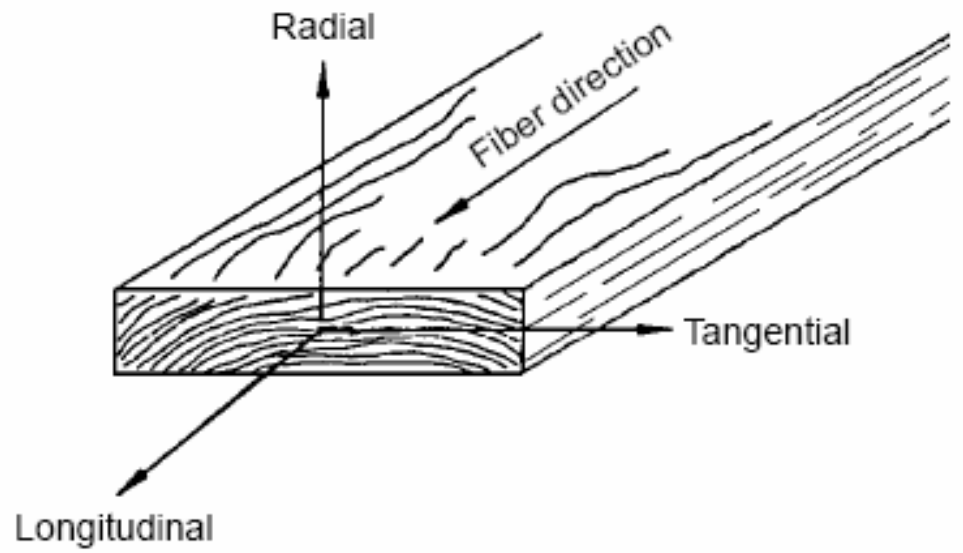

Figure 1-2 Principal axes with respect to grain direction and growth rings (Green et al. 1999)

\subsubsection{Moisture Content}

Moisture content is defined as the weight ratio of water to that of oven dry wood. Strength of wood is a function of moisture content. In a log, moisture content can vary from less then $10 \%$ to more than $200 \%$. The electromagnetic properties of wood (dielectric constant and conductivity) are a function of moisture content. These properties also differ from species to species and also vary within a given species from tree to tree. The effects of moisture content on the electromagnetic properties of wood are discussed in Section 1.4.

\subsection{ELECTROMAGNETIC PROPERTIES}

Two major electromagnetic (EM) properties which are of concern for this research are conductivity and dielectric constant, which are components of the complex dielectric permittivity. They are important because the velocity and attenuation of EM waves (produced by GPR equipment) depend on this factor.

\subsubsection{Conductivity}

This property of wood affects the transverse electrical field of EM signal, which is recorded by the GPR data acquisition system. Electrical conductivity of wood increases with moisture content. Change in electrical resistance with moisture content is shown in Figure 1-1. The shaded area indicates variability between different species. Conductivity (which is inversely proportional to electrical resistance) is greater along the grain than 
across the grain (Simpson and TenWolde 1999). Electrical conductivity is substantially increased in case of wood treated with preservative or fire-retardant or in presence of other electrolytic substances like salt.

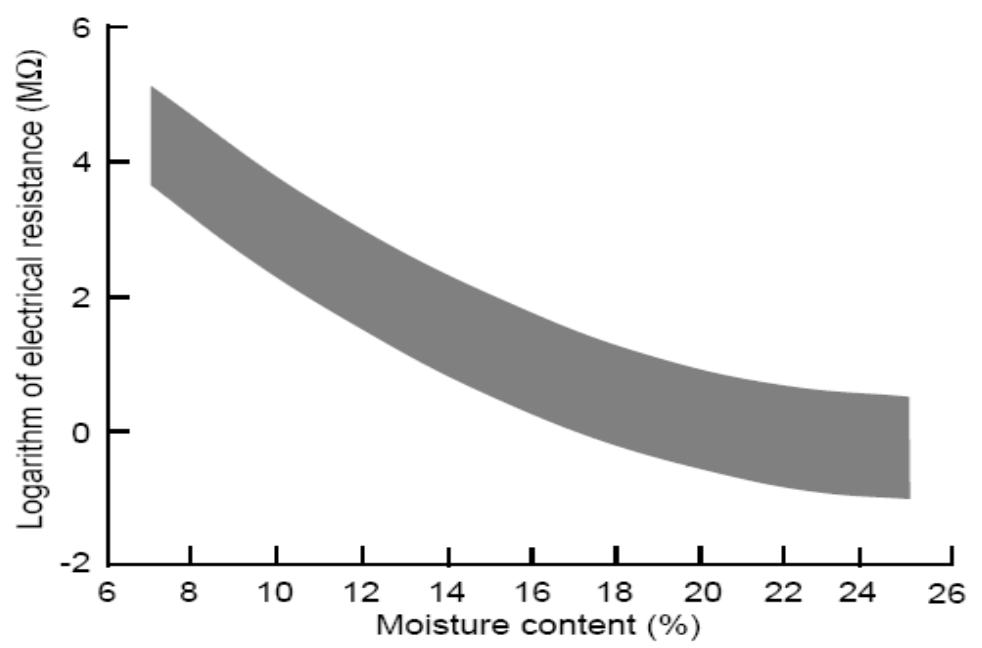

Figure 1-3 Change in electrical resistance with change in moisture content (Simpson and TenWolde 1999)

\subsubsection{Dielectric Constant}

Dielectric constant of a material is defined as the relative permittivity of a material. It is the ability of the material to carry alternating current as compared to that of vacuum. For dry wood the dielectric constant ranges from 2 to 5 , but increases sharply with moisture content and decreases slowly but steadily with increase in frequency of the applied electric field. Dielectric constant of wood also varies with orientation of wood (Simpson and TenWolde 1999). Dielectric constant as a function of moisture content and fiber orientation measured at $2.45 \mathrm{GHz}$ frequency for Tamarack is shown in Figure 1-4. The variation of dielectric constant with increase in frequency for Tamarack is shown in Figure 1-5. It is important to note that the variation with frequency and grain orientation is different for different species of wood. For example, some species may show highest dielectric constant in the longitudinal direction while others may show the lowest value in this direction (Afzal et al. 2003).

\subsubsection{Complex Dielectric Permittivity}

Complex dielectric permittivity of wood is described by two quantities: dielectric constant, $\varepsilon^{\prime}$ and loss factor, $\varepsilon^{\prime \prime}$. Loss factor measures the amount of electrical energy 
converted to thermal energy, and is related to conductivity and wave frequency. The relative complex dielectric permittivity, $\varepsilon$, is given by the following equation:

$$
\varepsilon=\varepsilon^{\prime}+i \varepsilon^{\prime \prime}=\varepsilon^{\prime}+i\left(\frac{\sigma}{\omega \varepsilon_{0}}\right)
$$

where,

$$
\begin{aligned}
& \sigma=\text { conductivity } \\
& \begin{array}{r}
\begin{array}{c}
\omega= \\
\text { angular frequency (radians } / \mathrm{sec})
\end{array} \\
=2 \pi * \text { cyclic frequency }(\mathrm{Hz})
\end{array} \\
& \begin{array}{r}
\varepsilon_{0}=\text { Dielectric permittivity of vacuum } \\
=8.854 \times 10^{-12} \text { farad } / \mathrm{m}
\end{array}
\end{aligned}
$$

The loss tangent is given by the following equation:

$$
\tan \delta=\frac{\varepsilon^{\prime \prime}}{\varepsilon^{\prime}}=\left(\frac{\sigma}{\omega \varepsilon_{0} \varepsilon^{\prime}}\right)
$$

The variation of loss tangent as a function of frequency and moisture content are shown in Figure 1-6 and Figure 1-7. Fiber Saturation Point (FSP) is the maximum cell wall moisture content the log can achieve. The FSP value is around 30\% (Simpson and TenWolde 1999). Dielectric constant of wood increases with moisture content whereas, loss factor increases up to the FSP and then gradually decreases with moisture content. The effect of moisture content on dielectric constant of different species at $2.38 \mathrm{GHz}$ is shown in Figure 1-8. Other factors that influence dielectric permittivity are: tree species, bulk density and temperature. 


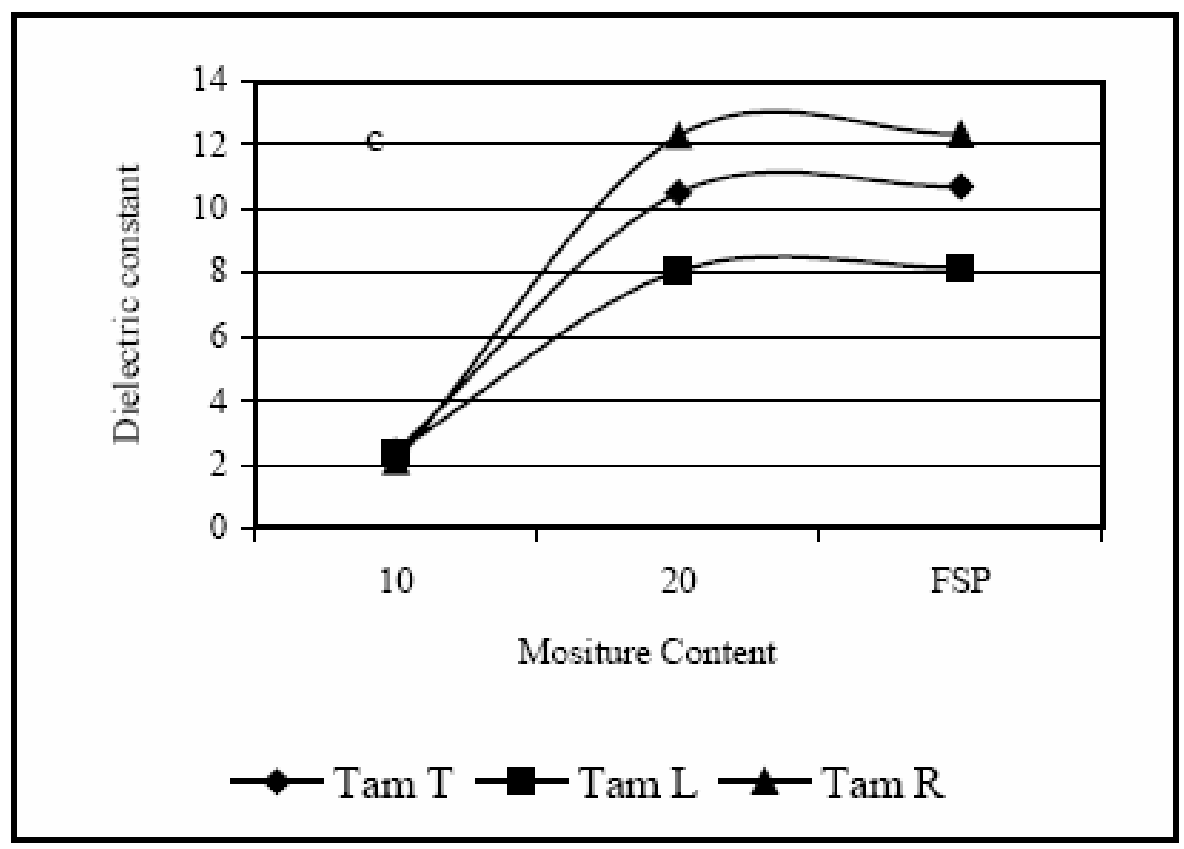

Figure 1-4 Dielectric constant as a function of moisture content measured at $2.45 \mathrm{GHz}$ for tangential (T), longitudinal (L) and radial (R) directions for Tamarack (Afzal et al. 2003)

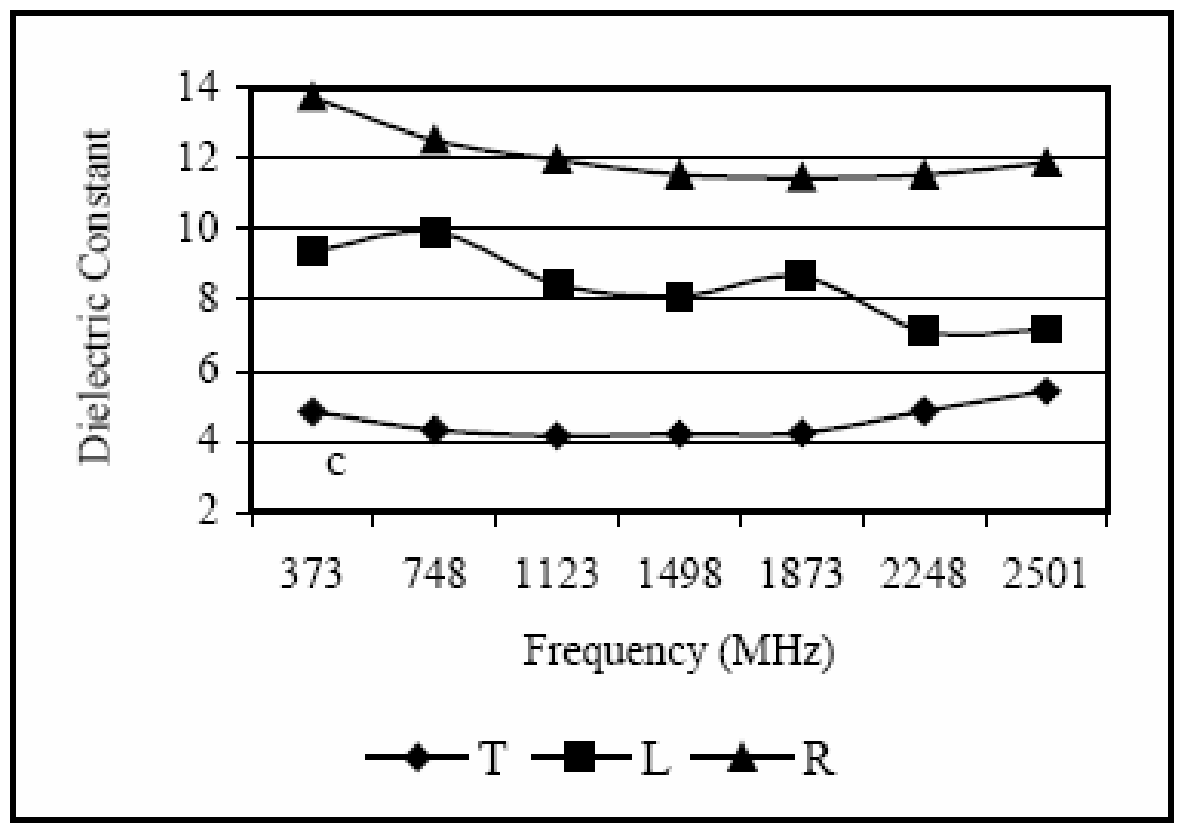

Figure 1-5 Dielectric constant as a function of frequency for tangential (T), longitudinal (L) and radial (R) direction for Tamarack (Afzal et al. 2003) 


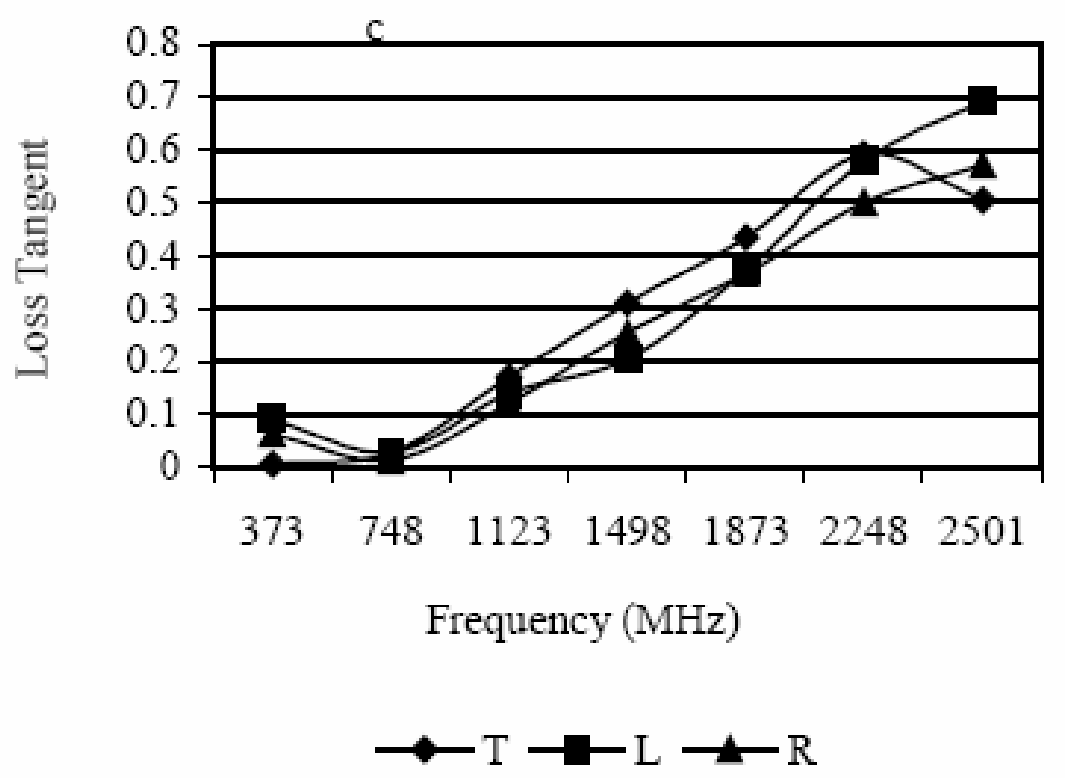

Figure 1-6 Loss tangent as a function of frequency for tangential (T), longitudinal (L) and radial (R) direction for Tamarack (Afzal et al. 2003)

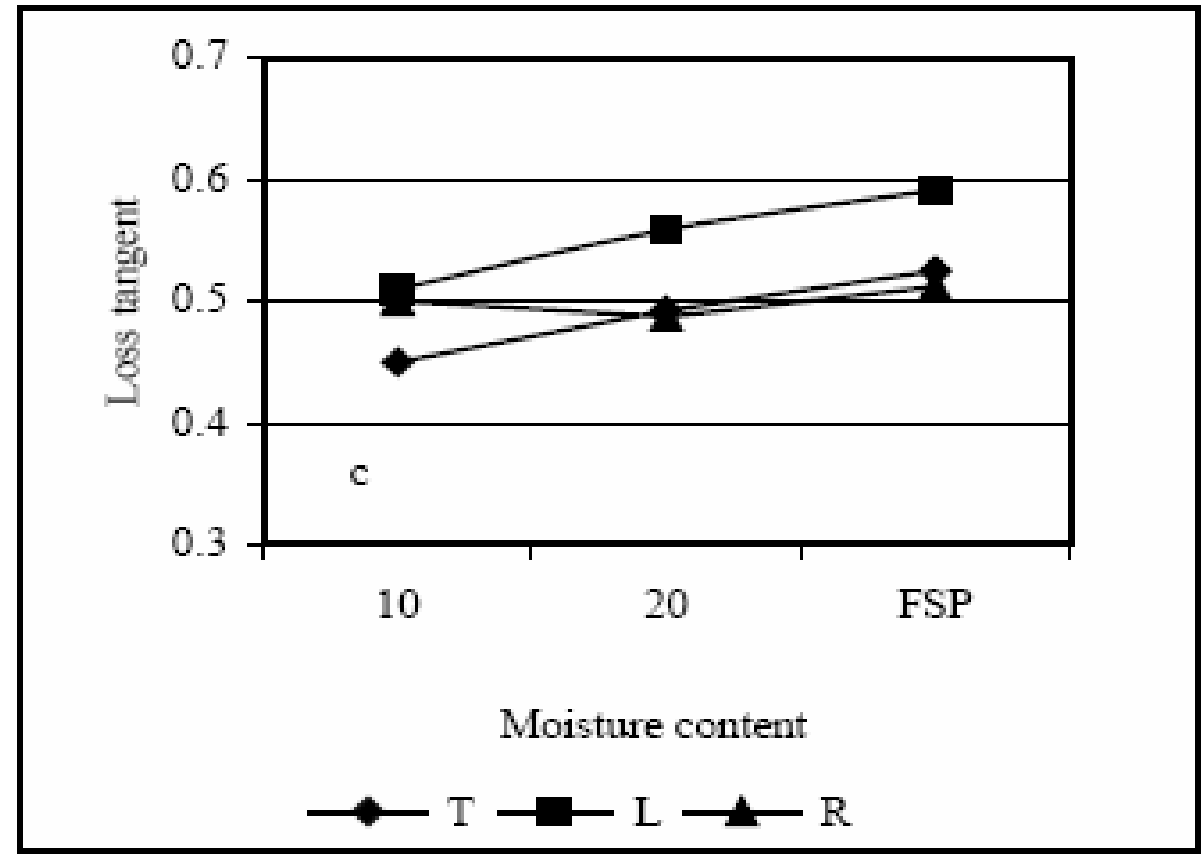

Figure 1-7 Loss tangent as a function of moisture content for tangential (T), longitudinal (L) and radial (R) direction for Tamarack (Afzal et al. 2003) 


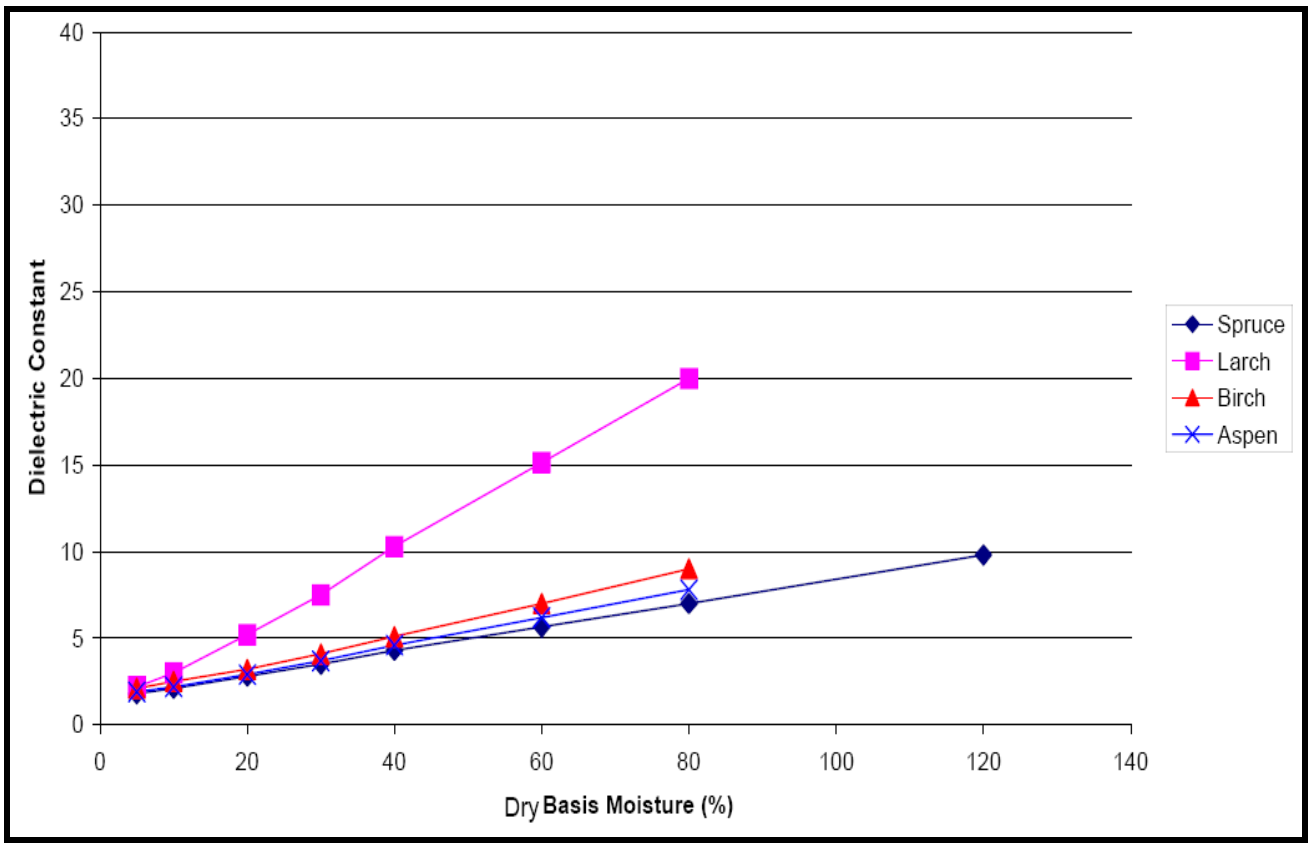

Figure 1-8 Dielectric Constant as a function of moisture content in the transverse direction for different species at frequency $2.38 \mathrm{GHz}$ (Keam Holdem Associates Ltd. 1999)

\subsection{NATURAL CHARACTERISTICS AFFECTING MECHANICAL PROPERTIES}

The natural growth characteristics of wood affect its properties. Natural defects such as knots and decays affect the mechanical properties and value of timber products.

\subsubsection{Knots}

Knots are generally the portion of a branch that remain in the tree after the branch dies and falls off or is manually cut. They are considered as a major defect in lumber, adversely affecting their use in construction and other kinds of applications. The fibers around the knot are distorted, which in turn lower the strength of wood. The reduction in strength is directly proportional to the size of a knot. Knots can be classified as sound and unsound. An unsound knot is generally rotten. Location of knots is the most important piece of information required for grading of lumber. The accurate location of knots is very important for modifying the sawing patterns to increase the lumber value. The knot on the surface indicates a knot in the underlying wood but this does not provide information regarding amount of fiber distortion or size of the knot inside (Green et al. 1999). The external and the internal knots are shown in Figure 1-9. 


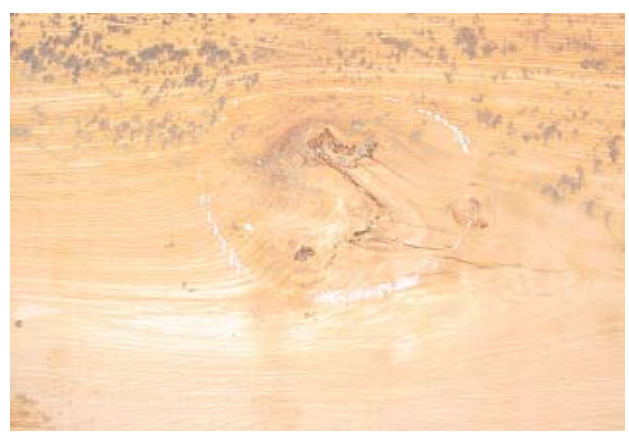

(a)

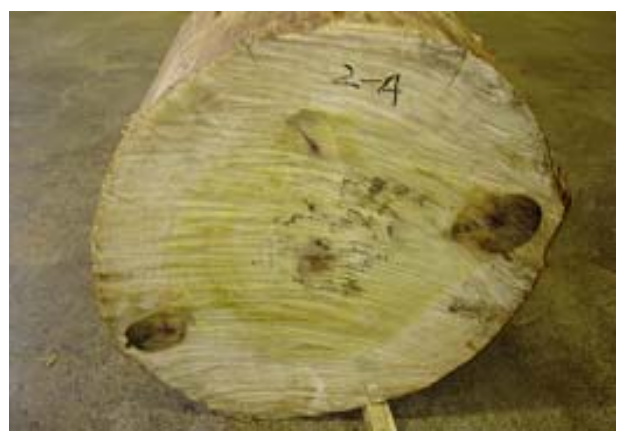

(b)

Figure 1-9 Knots (a) external (b) internal

\subsubsection{Decay and Rot}

Decays or rots affect the quality and grade of wood. Decays are generally located in the interior part where the moisture content is higher (Figure 1-10). Wood decay is primarily due to fungi, insects, bacteria, and marine bores. The fungi survive on organic materials provided by cell structure of wood. Temperature and moisture content play a very important role in the development of decay. The decay is slower at temperature below $10^{\circ} \mathrm{C}$ and above $35^{\circ} \mathrm{C}$. Also, the rate of decay is higher at moisture content above the fiber saturation point (average 30\%). Wood does not decay if kept dry.
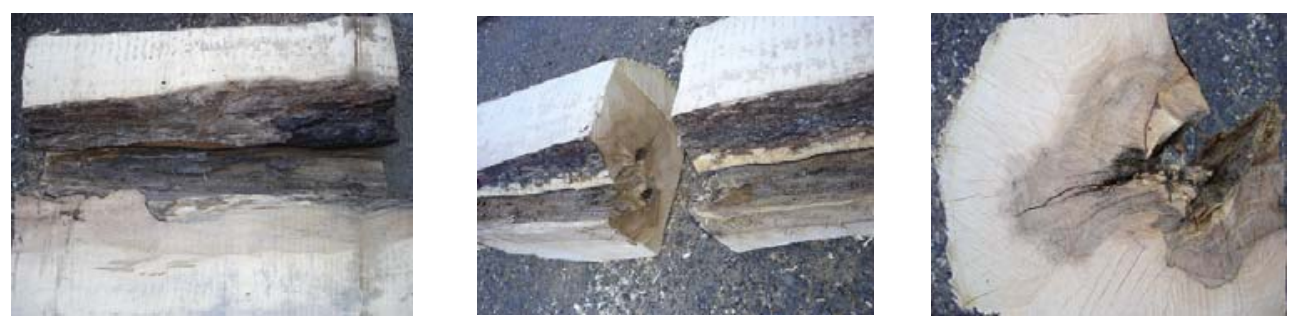

Figure 1-10 Decay or rot in wood

Discoloration of wood generally indicates early stages of fungal attack. Early stages of decay are very difficult to detect as compared to late stages which can be easily recognized. The decay can be classified into three different types: soft-rots, white-rots, and brown-rots. Soft-rots takes place during the initial stages of wood decay. They cause discoloration and stains and are very difficult to recognize. White-rots and brown-rots are major decay and can be detected more easily. The strength of wood is adversely affected 
by decay. Decay initially affects the toughness followed by reduction in strength. Lumber-grading is also affected by the presence of decay. Decay can be prevented by drying the wood and keeping the moisture content below $20 \%$ (Green et al. 1999).

\subsubsection{Embedded Metals}

Metals embedded by humans during the early stage of growth of the tree are one of the major problems faced by saw mills. Figure 1-11(a) shows an inserted metal rod. Figure 1-11(b) shows an embedded nail around which the tree has grown. Such nails constitute a major type of embedded metals. Embedded metals can damage the saw blade during sawing which results in significant downtime and increased maintenance cost. The presently available testing techniques such as metal detectors can detect the presence of metals but not the exact location of metals.

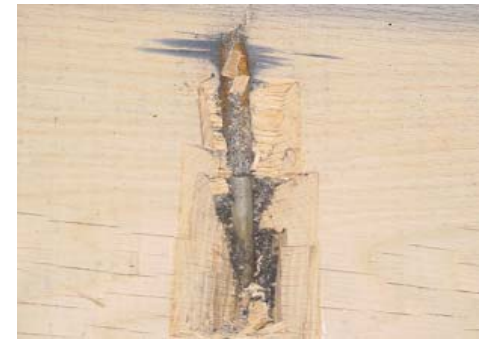

(a)

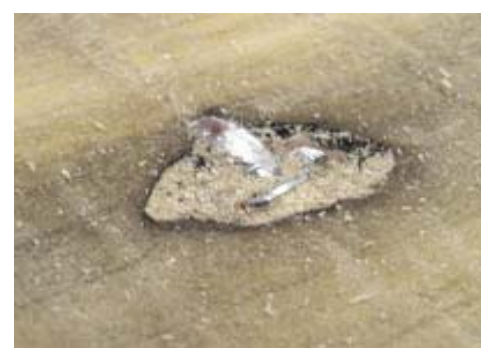

(b)

Figure 1-11 Embedded metal in wood

\subsection{ORGANIZATION OF THE THESIS}

This thesis investigates the use of Ground Penetrating Radar (GPR) for detection and characterization of subsurface defects in wooden logs. The research objectives and scope of this study were discussed earlier in this chapter. Fundamentals of GPR for assessment of logs are presented in Chapter 2. The GPR instrumentation setup for scanning logs is described in Chapter 3. Chapter 4 gives details of the procedure for basic data collection and data processing for logs. Chapter 5 describes the details of all the GPR experiments on logs and the results. The defects detected in this study include knots, rots, and embedded metals. The conclusions and recommendations are presented in Chapter 6. 


\section{Chapter 2 \\ GPR FUNDAMENTALS}

\subsection{OVERVIEW}

Ground Penetrating Radar (GPR) is a non-invasive scanning technique that can be used for mapping subsurface defects in concrete bridges, pavements, and wooden components. This thesis investigates the use of GPR for detection of sub-surface defects in logs. This chapter gives a brief overview of the basic fundamentals of GPR, the propagation of electromagnetic (EM) waves in logs, and the associated equations used for calculating the relative dielectric constant of logs. Also, this chapter briefly discuses the feasibility of using GPR for scanning logs and its advantages over other nondestructive techniques.

GPR emits pulses of radio-wave (EM wave) from the transmitter of the antenna into the structure or material under investigation. These pulses are reflected from subsurface interfaces or boundaries between materials with different dielectric constants (e.g, interface between good wood and defect) and received by the receiver of the antenna. The receiver may be the receiver of the same or a different antenna. These received pulses are converted into voltage signals and recorded as series of waveforms showing the amplitudes and arrival times of the echoes reflected from the interfaces. These waveforms are called radar-waveforms and are recorded as voltage vs. time as shown in Figure 2-1 (single waveform) and Figure 2-2(a) (series of waveforms). The display containing a series of recorded waveforms as the radar antenna is moved along the surface is often called a wiggle plot or waterfall plot.

Recent radar applications use graphic display of the scanned data. The received signals in the form of voltages are converted into grey scale or color plots with different values of voltage assigned different shades. This kind of display allows easy data analysis since the defects appear in the form of identifiable anomalies or discontinuities with different shades of grey scale or colors compared to surrounding defect-free areas. Example of such scans are given in Figure 2-2(b) and 2-2(c). The grey scale scans were used in this research since they were often easier to interpret compared to the color scans. 
Presently, GPR has been used largely for civil, geotechnical, and archaeological purposes (Bevan 1991, Maser and Roddis 1990, Halabe et al. 1997). GPR has been used for mapping roadways, structures and substructures. However, its use in the field of forestry has been limited. Its application to the field of wood technology is very new. To interpret the GPR data it is important to understand the fundamentals of EM signals and factors affecting transmitted and received signals.

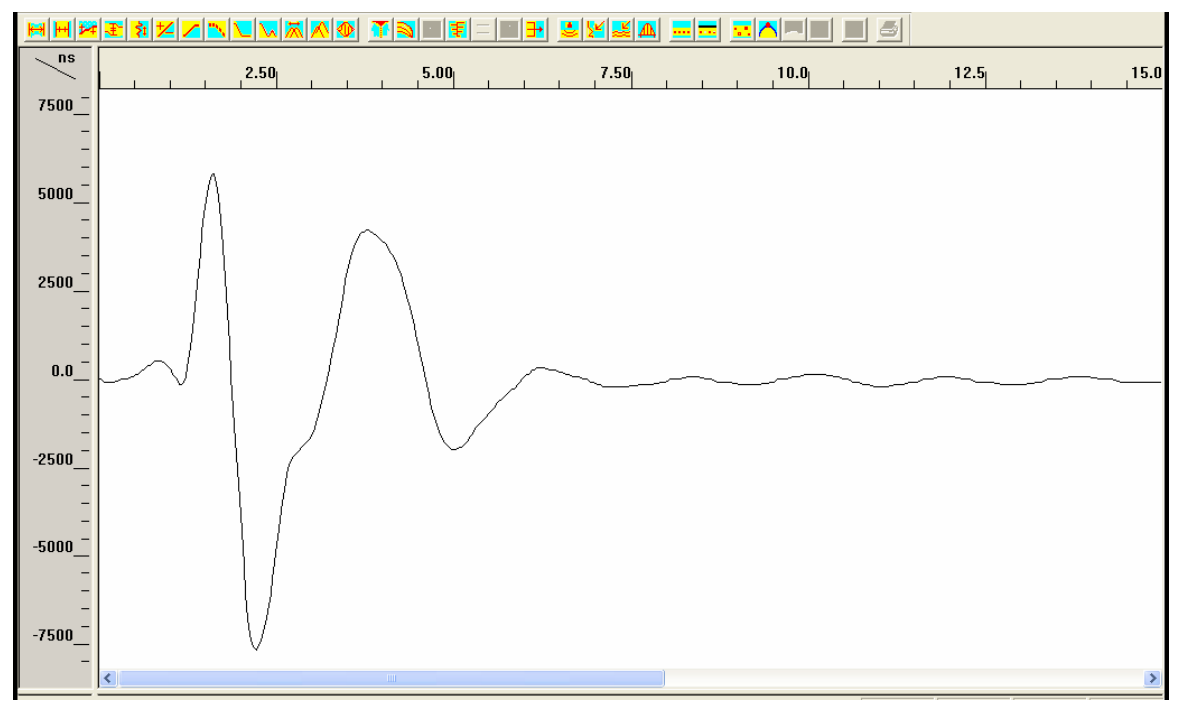

Figure 2-1 Single scan (extracted from sample of scans) with vertical axis showing the strength of the received signal in millivolts $(\mathrm{mV})$ and horizontal axis indicating the two way travel time within the material in nanoseconds (ns)

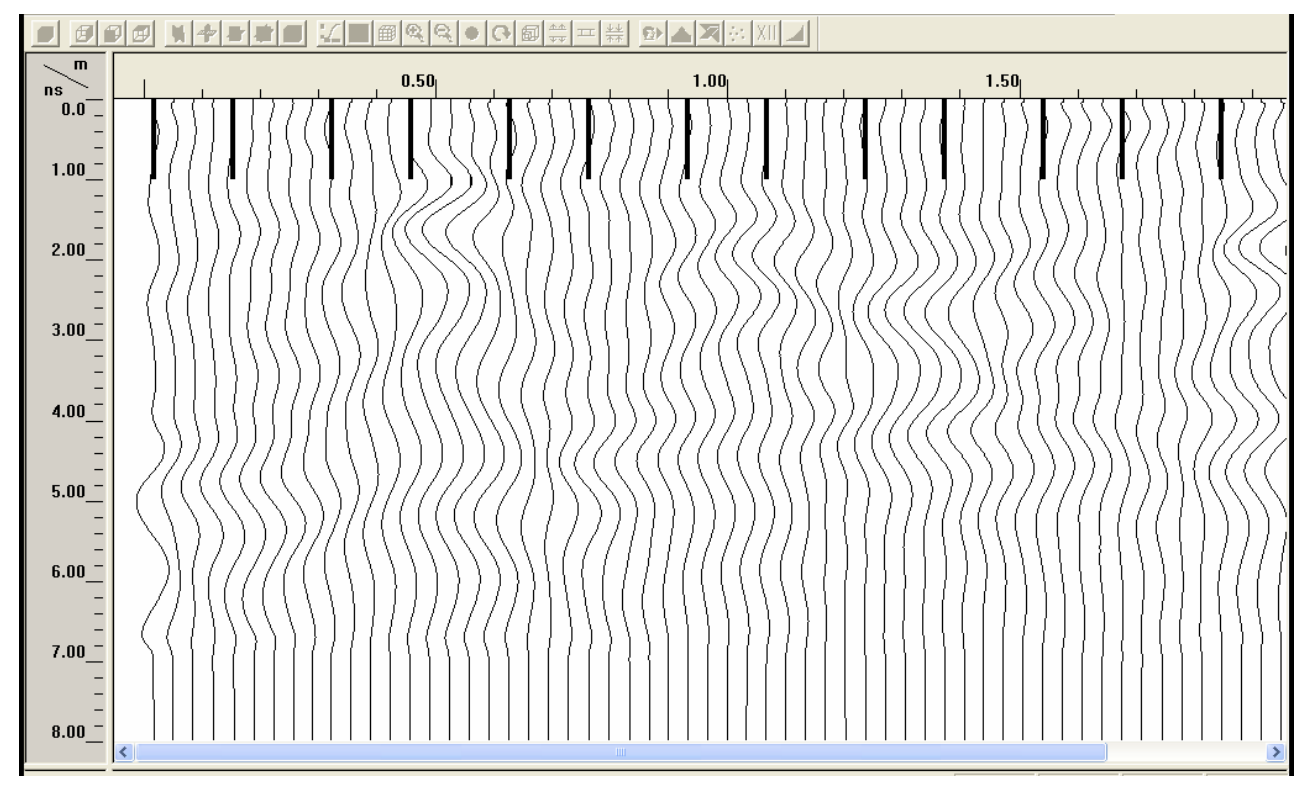

(a) 


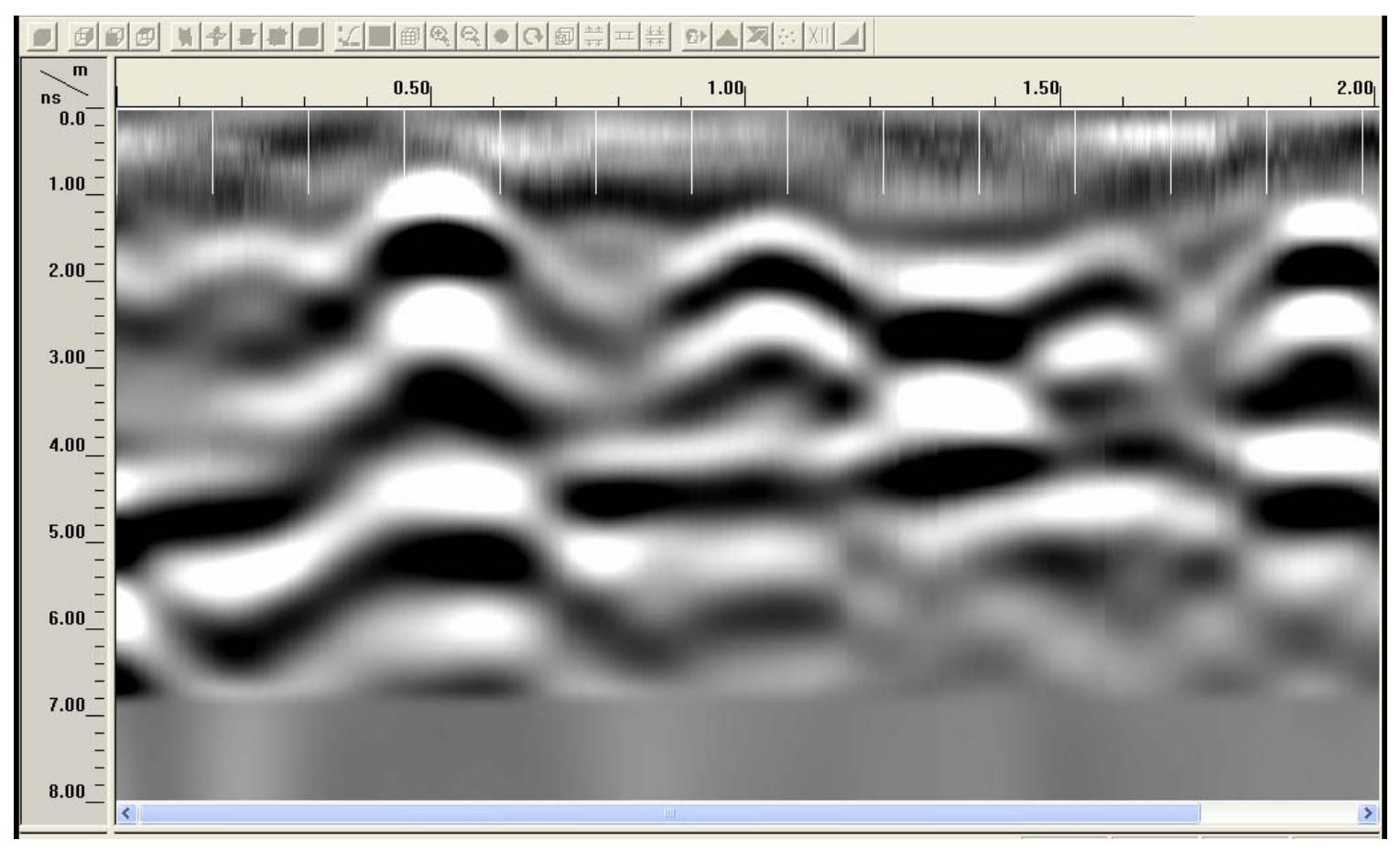

(b)

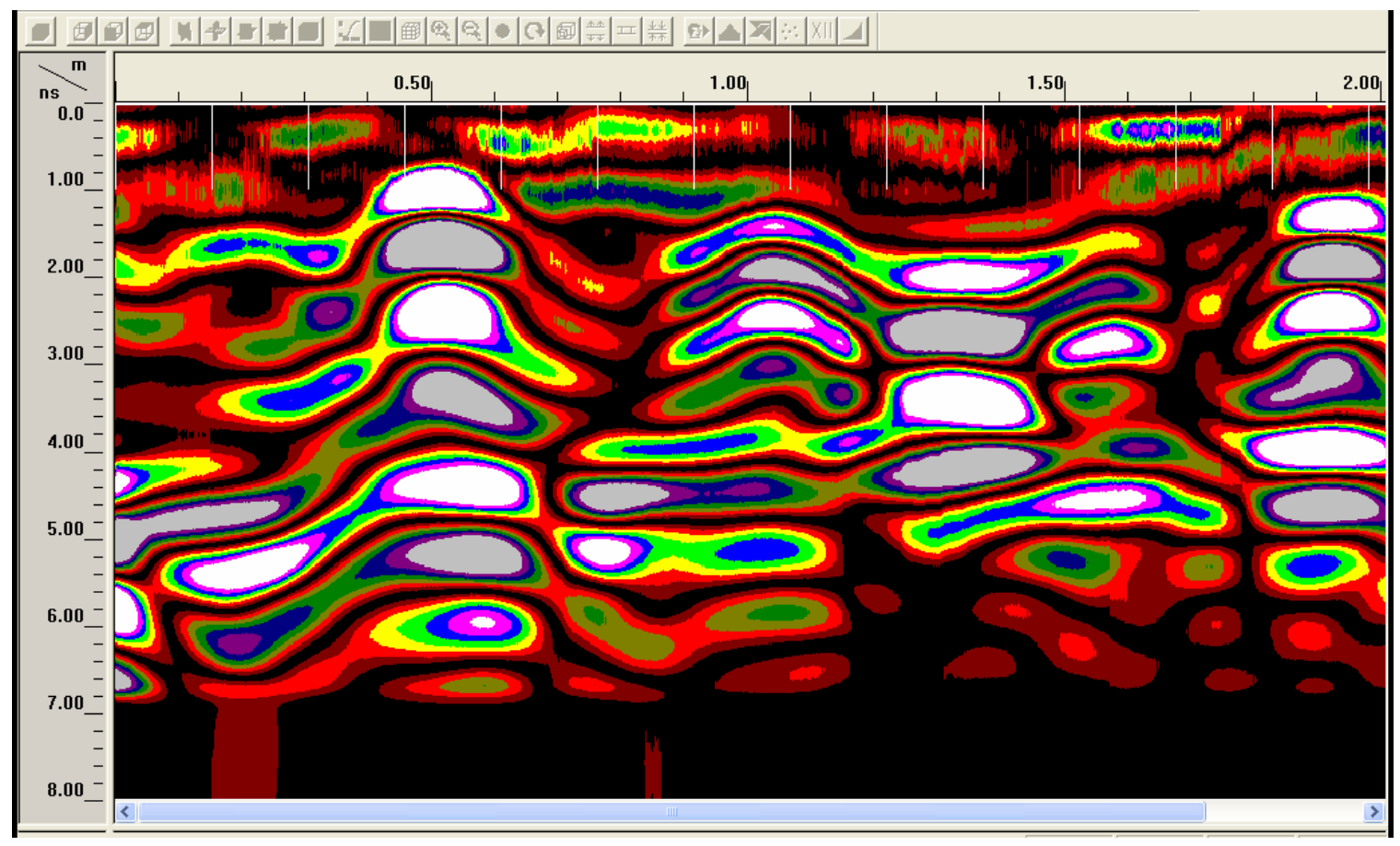

(c)

Figure 2-2 (a) Wiggle plot, (b) grey scale scan, and (c) color scan, of a wooden log, with vertical scale indicating the two-way travel time along the depth of the log in nanoseconds and horizontal scale indicating the travel distance or antenna position along the length of the log in meters 


\subsection{ELECTROMAGNETIC WAVES}

RADAR (Radio Detection and Ranging) is an application which utilizes the scattering of EM waves from an object for its detection and possible identification. Material properties like permeability, permittivity, and conductivity affect the propagation and reflection of EM waves. When an EM wave is sent into a structure, part of the wave is scattered or reflected from the interfaces between two dissimilar media (e.g., good wood and knot). The reflected waves are received and converted into interpretable waveforms. The amount of scattering is determined by surface shape and roughness and its material properties compared to the surrounding material, that is, the contrast in electromagnetic properties. For example, a metal sheet ideally reflects all the waves whereas a knot or decay would reflect part of the wave energy.

As the propagation of EM waves does not involve any mechanical vibration, they are not directly affected by the mechanical properties of materials such as stiffness, density or Poisson's ratio (Halabe et al. 1995); instead they depend on the electromagnetic properties of the material. The discontinuities in electromagnetic properties are considered as abnormalities or defects in the material. These defects appear in the form of signal anomalies in GPR scans. The intensity and shape of the observed anomaly can be attributed to some kind of defect within the log, such as knot, decay (rot), metal, or interfaces between wood of different densities.

\subsection{BASIC THEORY}

It is important to understand the electrical properties of wood before any kind of evaluation is done using EM waves. The velocity of the EM wave in logs depends on its complex dielectric parameters, which in turn are influenced by various other factors such as moisture content, presence of impurities, species, temperature, fiber orientation and frequency. The two main properties which influence the EM signals are conductivity and dielectric constant, which are components of the complex dielectric permittivity of the material as explained in Chapter 1. These properties are affected by moisture content and frequency as discussed in Chapter 1. 


\subsubsection{Conductivity}

Conductivity is the ability to carry current. Conductivity of wood affects the transverse electric field of EM wave (Annan 2001). It has a significant effect on the attenuation of the signal. Conductivity of dry wood is negligible but increases with increase in moisture content. Attenuation of EM signal increases with an increase in conductivity; and hence increasing moisture content would increase the wave attenuation, that is, the amount of power dissipated in the wood. Conductivity also increases with increase in impurities which may be in the form of chemicals added to prevent wood decay or act as fire retardants. In addition, the value of conductivity depends on the wave frequency, temperature, density, grain direction and species of wood.

\subsubsection{Dielectric Constant}

Dielectric Constant or permittivity of a material is the measure of its ability to resist the formation of electric field. The dielectric behavior of wood is affected by several factors; moisture content, wave frequency, temperature, density, grain direction, and species. The dielectric constant increases significantly with increase in moisture content.

The velocity of an electromagnetic wave in any material depends on the dielectric constant of the material. This wave velocity is governed by Equation 2.1, where $V_{0}$ is the velocity of light or any EM wave in vacuum $\left(=3 \times 10^{8} \mathrm{~m} / \mathrm{s}\right), \varepsilon^{\prime}$ is the dielectric constant of the material and $V$ is the velocity of EM wave in the material.

$$
V=\frac{V_{0}}{\sqrt{\varepsilon^{\prime}}}
$$

The GPR signal is recorded in the form of amplitude $(\mathrm{mV})$ versus time (ns). The time parameter is dependent on the wave velocity. Relative dielectric constant of the material can be calculated from Equation 2.1 if the velocity of EM wave in the material can be measured with respect to that of vacuum. The velocity of EM wave in the material can be obtained from Equation 2.2, where $d$ is the distance traveled by the EM wave in time $t$.

$$
V=\frac{d}{t / 2}
$$


The travel distance $(d)$ in this case is the diameter of the log measured at the point of velocity measurement. The two-way wave travel time $(t)$ through the log and back can be obtained from the GPR signal, and the factor of $1 / 2$ is used to obtain the one-way travel time through the $\log$. After computing $V$ from Equation 2.2, the dielectric constant of the $\log$ can be obtained using the Equation 2.1. However, since the moisture content and thus the dielectric constant of the log is varying over its thickness (lower value near the surface and higher value towards the center), the dielectric constant calculated from the above equations is the average dielectric constant over the entire cross-section and not the dielectric constant at a specific point.

\subsection{ATTENUATION OF ELECTROMAGNETIC WAVES}

The electromagnetic signal loses its energy as it passes through the medium. It is very difficult to quantify the amount of energy lost, until and unless a specific model is developed for the material. The factor which largely influences the wave attenuation is the material conductivity. Also, wood is a highly heterogeneous and anisotropic material which further increases the wave attenuation. The wave attenuation and conductivity in a material increases significantly with the amount of moisture content (Halabe et al. 1993, Halabe et al. 1995). The moisture content in wood may vary from $10 \%$ for dry wood to more then $150 \%$ to $200 \%$ for a wet, moist wood. Also, the higher the frequency of EM waves the higher is the wave attenuation (Halabe et al. 1993).

\subsection{REFLECTION AND TRANSMISSION OF RADAR WAVES AT AN INTERFACE}

It is very important to understand reflection and transmission of radar waves at an interface in order to interpret GPR signals and detect subsurface defects or change in wood characteristics. There are at least two interfaces for logs, one at the top surface (antenna-wood interface) and the other at the bottom surface (wood-air interface). In addition, there could be internal interfaces due to presence of knots, decays, and embedded metals.

The electromagnetic signal can be decomposed into transverse electric field (TE) and transverse magnetic field (TM) and this decomposition is strictly a function of the 
interface geometry (Annan 2001). When the incidence of EM wave is vertical there is no distinction between TE and TM.

The amplitude reflection coefficient, $\mathrm{R}_{1,2}$ is given by Equation 2.3 , where 1 and 2 denote the first and second media at the interface and $\varepsilon_{1}^{\prime}$ and $\varepsilon_{2}^{\prime}$ are the dielectric constants of the two media (Halabe et al. 1993, Halabe et al., 1995).

$$
R_{1,2}=\frac{\sqrt{\varepsilon_{1}^{\prime}}-\sqrt{\varepsilon_{2}^{\prime}}}{\sqrt{\varepsilon_{1}^{\prime}}+\sqrt{\varepsilon_{2}^{\prime}}}
$$

The amplitude transmission coefficient, $\mathrm{T}_{1,2}$ is given by Equation 2.4 as,

$$
T_{1,2}=\frac{2 \sqrt{\varepsilon_{1}^{\prime}}}{\sqrt{\varepsilon_{1}^{\prime}}+\sqrt{\varepsilon_{2}^{\prime}}}
$$

As per Equation 2.3, if the dielectric contrast between the two media is high (e.g., wood and embedded metal), the reflection coefficient is high, which results in a strong reflection or echo signal. On the other hand, defects such as knots would result in lower amplitude reflections. Also, as per Equation 2.3, the reflection from antenna-wood interface (low to high dielectric medium) is negative while that at wood-air interface (bottom of the $\log$ ) is positive. This means, the bottom reflection (and the reflection from the defects) are of opposite sign $\left(180^{\circ}\right.$ phase shift $)$ compared to the top surface reflection.

\subsection{EFFECTIVE RANGE AND RESOLUTION}

The range of the system depends on the frequency of the EM wave from the transmitter. For the same GPR system, higher frequency EM signal would result in shallower penetration depth than with an antenna with a lower frequency (Belli 2002). This is because wave attenuation increases significantly with increase in frequency.

Resolution is the measure of how far two abnormalities have to be from each other to be differentiated, and it is a function of the wavelength or the frequency of the transmitted EM wave (Belli 2002). Two defects are very difficult to differentiate if they are not separated by a wavelength, or at least half the wavelength. Wavelength $(\lambda)$ of an EM wave is related to its frequency $(f)$ in accordance with the following equation. 


$$
\lambda=\frac{V}{f}
$$

Here $V$ is the wave velocity in the material obtained using Equation 2.1. For a $900 \mathrm{MHz}$ frequency transmitter, the wavelength in air is $0.333 \mathrm{~m}$. For relatively dry wood with a dielectric constant $\left(\varepsilon^{\prime}\right)$ of 9 , the wavelength would reduce to $0.111 \mathrm{~m}$, whereas for moist wood with $\varepsilon^{\prime}$ of 16 , the wavelength would be $0.083 \mathrm{~m}$. For the latter case, assuming the depth resolution to be half the wavelength, the resolution would be $0.0415 \mathrm{~m}$. With higher frequency, it is possible to lower the wavelength and achieve better resolution, however, the attenuation of the EM wave would increase and penetration depth would decrease. Usually logs have high moisture content, which results in high signal attenuation. The $900 \mathrm{MHz}$ transmitting antenna provides an optimal frequency for penetrating the entire depth (diameter) of the log and provides reasonably good resolution. Higher frequency antennas did not provide the desired penetration depth. This was the reason that $900 \mathrm{MHz}$ frequency antenna was selected for this research.

\subsection{EFFECT OF DEFECTS ON RECEIVED SIGNALS}

The defects can be detected from the reflection of the transmitted EM pulse from the boundary or interface between the defect-free wood and the defective region. The defects or complexities have a different dielectric constant from that of surrounding wood. The amount of signal reflected by the defect and transmitted through the defect depends on the reflection and transmission coefficients given by Equations 2.3 and 2.4. Another means of detecting defect relies on wave attenuation. A signal traveling trough a defect such as decay would attenuate significantly, resulting in lower reflection from the bottom of the log. The clarity with which the defect can be detected depends on the amount of attenuation of the signal. The amount of signal attenuation also depends on the size and orientation of the defect. Generally, embedded metals are very small in size

compared to knot and decay. Though metals are small in size, they have a very high reflection coefficient leading to strong echo signals.

\subsection{GPR AS A PREFERED TECHNIQUE}

Presently there are no scanning technologies to accurately map the defects in logs. In order to address defects in logs, the nondestructive scanning technology must be 
capable of assessing internal condition of logs before they are sawn into lumber. Also, for on-line implementation in a factory setting, the scanning technique must have the capability of collecting and processing data in real-time. Techniques using stress wave or ultrasound can detect knots and rots accurately, but are too slow in gathering data as the sensors have to be in contact with logs for testing (Halabe et al. 1995, Halabe et al. 1996, Schad et al. 1996). Thus, these techniques are totally unsuitable for real-time on-line implementation in saw mills. On the other hand, ground penetrating radar provides higher rate of data collection and faster processing. The present GPR system manufactured by GSSI has the added advantage that data collection and processing can be done simultaneously thus making it the best nondestructive scanning technology for on-line implementation. Other techniques like Nuclear Magnetic Resonance (NMR), X-ray, and ultrasonic tomography techniques (Schad et al. 1996, Ross et al. 1998) have several disadvantages. For example, these techniques are slow in data acquisition or processing or both making them unsuitable for on-line implementation. Also, techniques like transverse vibration techniques and camera vision systems can be used only on processed lumber of smaller thickness and not directly on logs. Also, these techniques lack user friendliness and do not provide direct imaging system whereas, GPR provides a more user friendly processing tools and imaging system. However, significant research needs to be conducted to determine and develop suitable measurement configuration for on-line implementation. Use of such an on-line system could significantly increase the productivity of lumber and also decrease the maintenance cost.

\subsection{LITERATURE REVIEW ON USE OF GPR FOR WOOD INSPECTION}

In spring of 1985, Detection Sciences, Inc. (1994) demonstrated the feasibility of inspecting wooden beams using impulse radar. Few beams in Hasbro Toy Factory in Pawtuckett, Rhode Island were tested using impulse radar. The radar system was capable of detecting internal rot that was invisible to naked eye. A small hand held radar antenna with center frequency $900 \mathrm{MHz}$ was used for this purpose. It was concluded that uniform wood with no defects would result in a relatively uniform travel time. On the other hand, portions of the wood with decay or rot would show an increase in the travel time and hence the transmitted pulse would result in a non-uniform signal output. 
Canpolar, Inc. (1987), conducted some tests for preliminary assessment of Impulse Radar to detect decay in hard wood. A-cubed pulseEKKO I impulse radar system with a center frequency of $700 \mathrm{MHz}$ was used to scan twelve bolts of Aspen with diameter of 0.1 to $0.12 \mathrm{~m}$. The electrical characteristic of defect-free and decayed wood produced different radar response. Decayed sample gave increased ringing (echoes) in the radar signal.

In 1994, Detection Science and Forest Product Laboratory (FPL) explored nondestructive testing of wood using GPR. For this purpose ground coupled antennas with center frequency of $1.2 \mathrm{GHz}$ was used (Detection Sciences, Inc. 1994). Sixteen specimens of $1.22 \mathrm{~m}$ length and $0.3 \mathrm{~m}$ diameter were prepared by FPL and tested in 3 longitudinal planes for investigating defects and interaction of radar with log specimens. It was recognized that uniform, high grade logs can be easily recognized in contrast to logs having more knots and internal defects.

Muller (2002), investigated timber girders from a demolished bridge and girders on existing four span timber bridge using GPR. These girders had circular cross-section with diameter of around $0.35 \mathrm{~m}$ to $0.4 \mathrm{~m}$ and the EM wave range (two-way travel time) for the GPR recorded data was $11 \mathrm{~ns}$. The GPR testing was conducted using ground coupled dipole antenna with central frequency of $1.2 \mathrm{GHz}$ manufactured by Geophysical Survey Systems, Inc. (GSSI). Other NDT techniques like ultrasonic and gamma ray transmissions were also used for investigation. Of the techniques used GPR was found to be the most reliable NDT method for assessing internal defects in wood.

The above literature review indicates that extensive research is still needed in terms of mapping the extent of subsurface defects. Most of these references deal with detection of advanced stages of decay, but for saw mills it is important to detect decays at early stages as well. Also, only one of the available references has dealt with knots and none of them have dealt with detection of embedded metals. In addition, the fresh logs considered in this study pose additional challenge because of their larger diameter $(\sim 0.8$ m) and high moisture content ( $100 \%$ at the center of the log). Testing of such logs require a GPR system capable of adequate penetration without much loss in resolution. The system used in this study is described in the next chapter. 


\section{Chapter 3 \\ EXPERIMENTAL SETUP}

\subsection{GPR SYSTEM}

The GPR system used for this particular research is Subsurface Interface Radar (SIR) System, manufactured by Geophysical Survey System, Inc. (GSSI). The GPR equipment utilized for assessing the condition of logs consists of a data acquisition system (DAQ), two antennas, and a portable antenna deployment frame with an attached survey wheel. The data acquisitions system houses the control unit and storage and display devices. The transmitter and receiver are both incorporated in the same antenna, and both antennas are identical. The general setup of the scanning arrangement is shown in Figure 3-1.

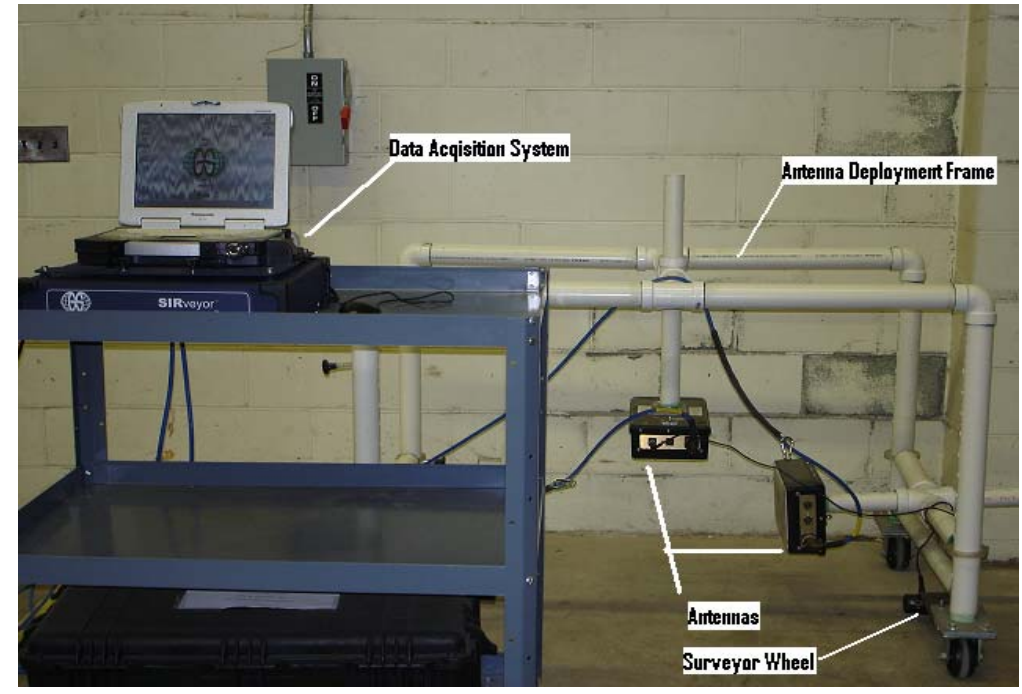

Figure 3-1 General setup of the GPR data acquisition system

\subsubsection{Data Acquisition System}

The data acquisition system, shown in Figure 3-2, consists of rugged, powerful SIR-20 two channel data acquisition unit controlled by a Windows based portable laptop computer with GSSI's Windows-based RADAN post-processing software (GSSI 2005). Table 3.1 gives the specifications of the SIR-20 system used in this research. The data acquisition system controls operations to perform the data acquisition and processing, and display the measured and processed data. While this system had the capability to collect 
data at a rate of $800 \mathrm{scans} / \mathrm{sec}$, new regulations imposed by the U.S. Federal Communications Commission (FCC) have led to system modification with a maximum scanning rate of $153 \mathrm{scans} / \mathrm{sec}$. This is not a problem for scanning logs where a scan rate of 75 to $100 \mathrm{scans} / \mathrm{sec}$ is quite adequate. The GPR system also allows the operator to collect radar data in single line mode or 3D format. However, the 3D format is currently available only for planar objects like bridge decks, and not for objects with circular crosssection such as logs. The SIR-20 data acquisition system has several desirable features: it has two-channel high speed data collection unit with additional channels for external markers, if desired; it has both data acquisition and processing capabilities in the same unit, making the interpretation very fast; and it is GPS compatible (GSSI 2005). Additional display and post-processing features are available in the radar analysis software, RADAN, which is quite user friendly.
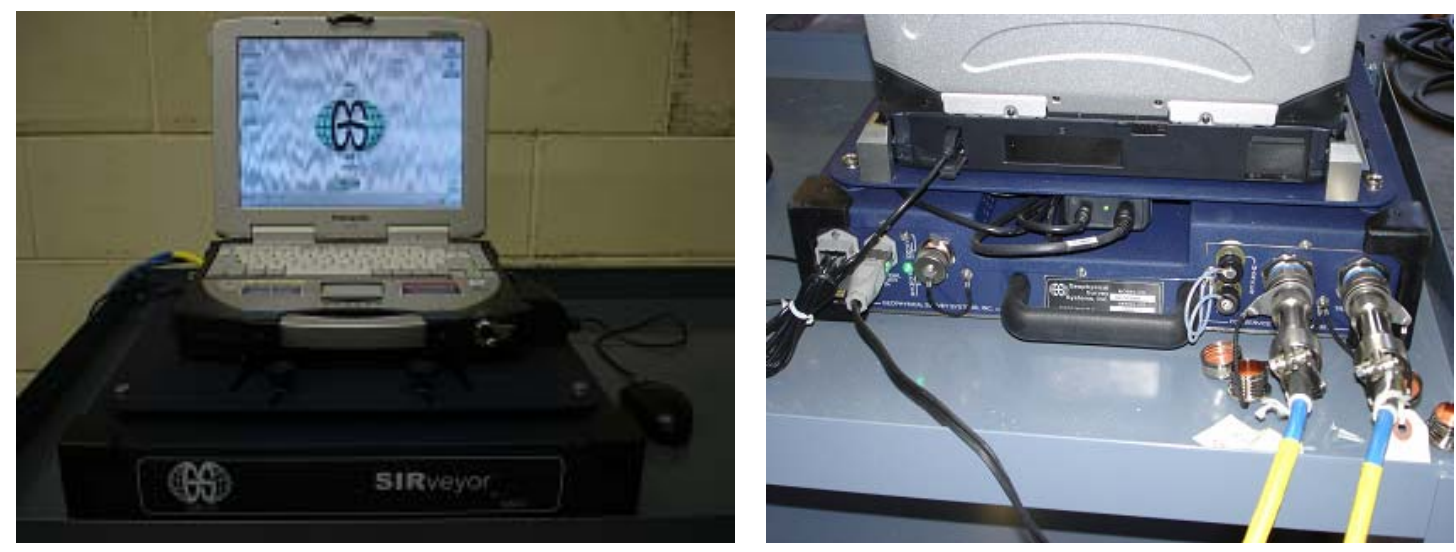

Figure 3-2 Data acquisition system (laptop computer and main frame)

\subsubsection{Antenna}

In general, there are three categories of GPR antennas, ground coupled, air launched, and borehole antennas. GSSI provides a wide range of antennas under these three categories for different applications as shown in Table 3.2. All the antennas are mono-static (both transmitter and receiver on one electronic plug-in in a single antenna housing) but can be also used in a bi-static mode where one antenna transmits the signal and the other one receives it. Ground coupled antennas provide deeper penetration (compared to air launched ones) because they are coupled to the material surface. Moreover, most ground coupled antennas also have lower frequencies $(<1000 \mathrm{MHz})$, 
resulting in larger penetration depths. On the other hand, air launched antennas $(\sim 1$ to 2 $\mathrm{GHz}$ frequency), which are mounted at least 6 inches above ground can only be used for shallow penetration (e.g., 6" to 10" thick concrete bridge deck), but provide higher resolution. Since the ground-coupled antennas have higher penetration and longer scanning ranges, they have slower collection rate than air-coupled antennas (Belli 2002). For this research, two GSSI Model 3101D $900 \mathrm{MHz}$ antennas were used (Figure 3-3). These antennas were chosen because these were ground coupled mono-static antennas, which could provide penetration depths of up to $1 \mathrm{~m}$ even in moist logs and at the same time provide the best possible resolution for this penetration depth.

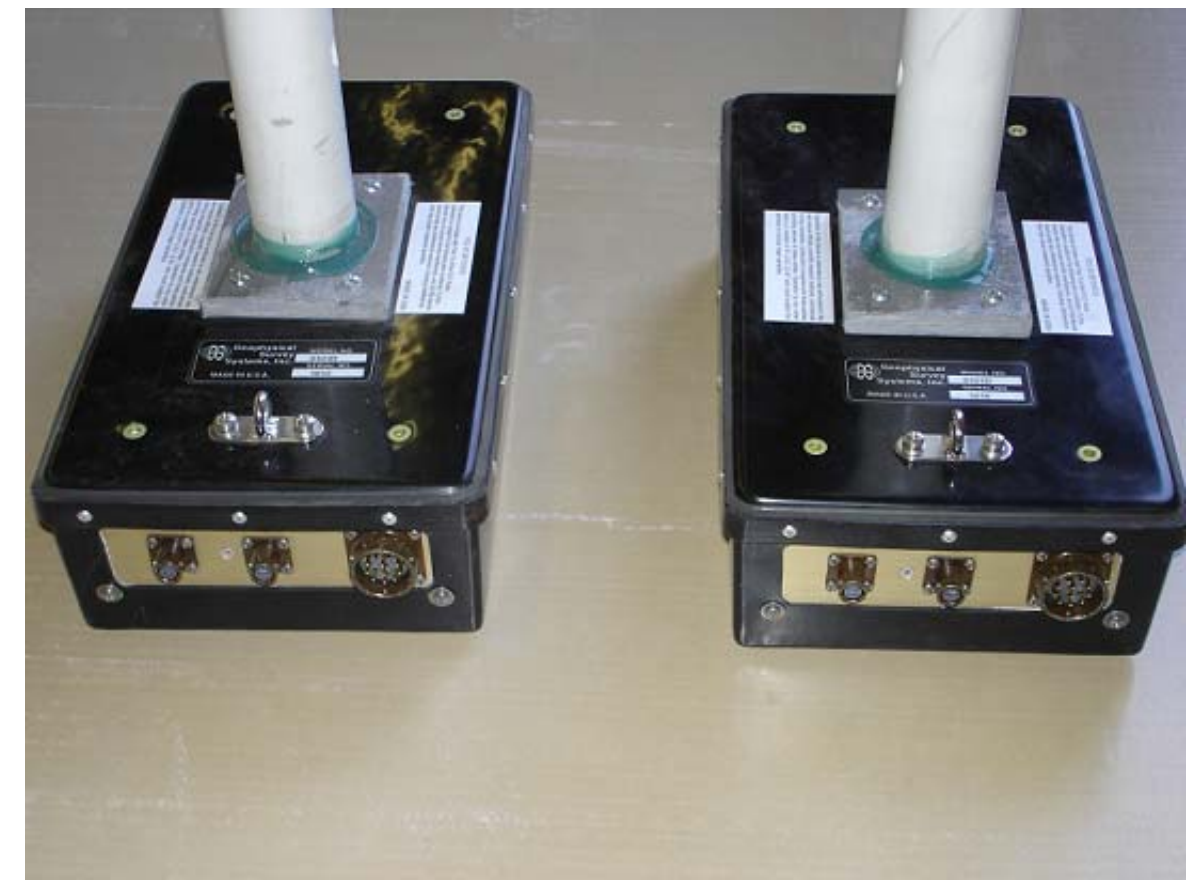

Figure 3-3 Two $900 \mathrm{MHz}$ antennas

\subsubsection{Antenna Deployment Frame}

The antenna deployment frame as shown in Figure 3-4 was specially designed using polyvinyl chloride (PVC) pipes and other fiber reinforced polymer (FRP) materials to avoid the interference of metal with EM wave energy. The only metallic components were the aluminum plates used for mounting the molded plastic wheels. Non-swiveling wheels were used to prevent side sway of the frame during data collection. The top antenna could only be moved in the vertical direction. However, the antennas on the side could be adjusted in both the horizontal and the vertical direction, depending on the log 
diameter. This frame provided versatility for scanning logs of diameters up to $0.7 \mathrm{~m}$. The different configuration in which the antenna(s) could be placed is shown in Figure 3-5.

The survey wheel was fixed to one of the legs at the lower end of the frame as shown in Figures 3-1 and 3-4. The survey wheel includes an optical encoder which transmits distance information to the GPR data acquisition system using a connecting cable. The survey wheel allows data collection only in the forward direction. The other way to collect data is in the free run mode without the survey wheel, but this mode does not provide any distance information. Therefore, all the data was collected in the survey wheel mode. In this mode, data is collected based on the rotation of the survey wheel with the sampling rate set by the user. There would be no data collection if there is no rotation of the survey wheel.

\subsection{OTHER ACCESSORIES}

In order to rotate the logs for scanning, a set of rollers (Figure 3-6) were manufactured. These rollers are adjustable to accommodate small and large diameter logs. A can-hook was used to lift and spin logs on the rollers. An axe (Figure 3-7) was used to trim the knots so the log would spin smoothly and also to ensure that knots did not cause an obstruction for the antenna movement close to the log surface. To simulate embedded metals, $3 / 8$ " $(9.5 \mathrm{~mm})$ and $5 / 8$ " $(16 \mathrm{~mm})$ diameter iron rods with lengths of 3" - 4" (76 - $100 \mathrm{~mm})$ were inserted in some of the logs after drilling holes with an extra long drill bit. An 18" (0.46 m) chain saw (Figure 3-7) was used for cutting some of the long logs before scanning. The chain saw was also used for sawing the logs after GPR scanning in order to observe the internal defects for correlating with the anomalies in the GPR scans. A crane or a lift (Figure 3-8) was used to lift the logs and place them on the rollers. 


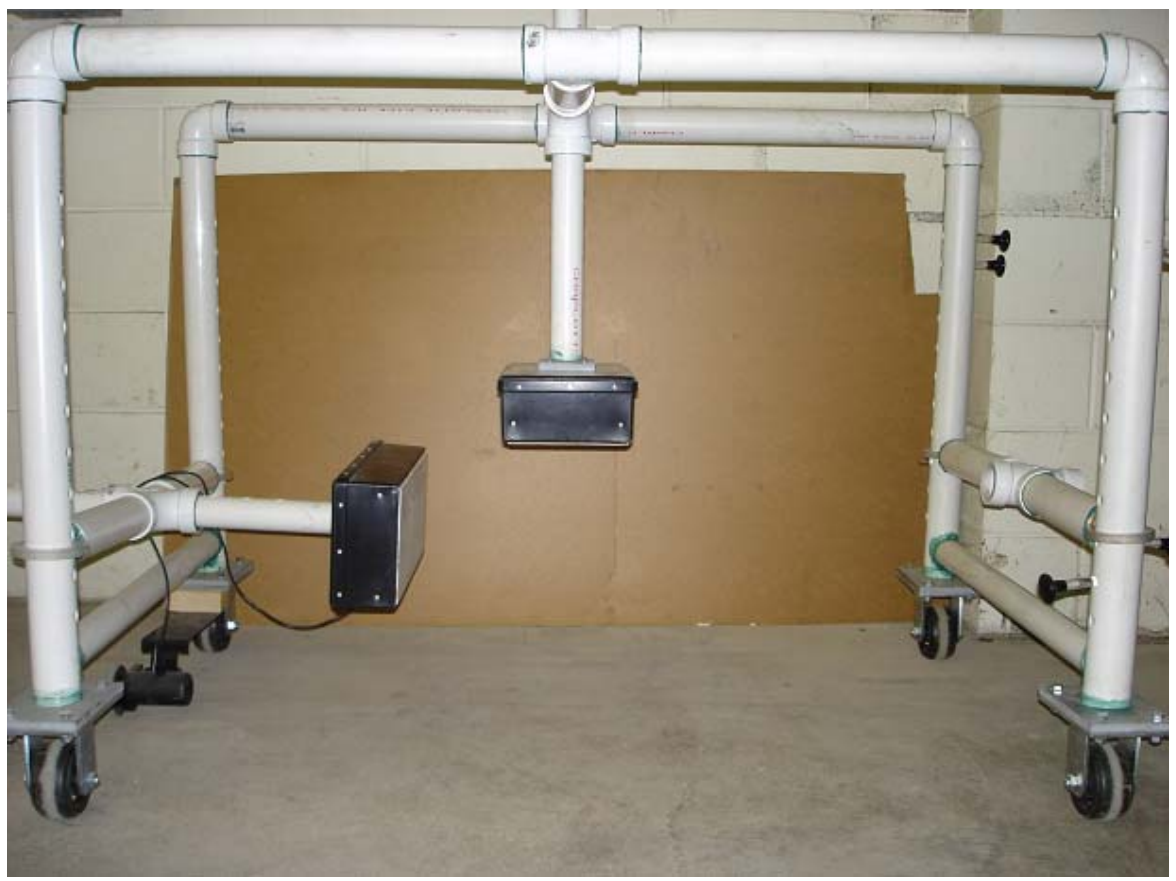

Figure 3-4 Antenna deployment frame
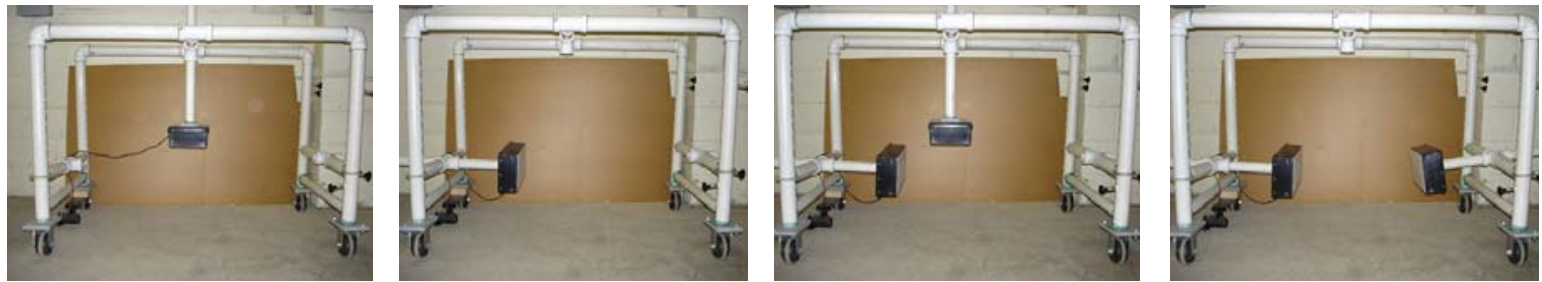

Figure 3-5 Antenna configurations
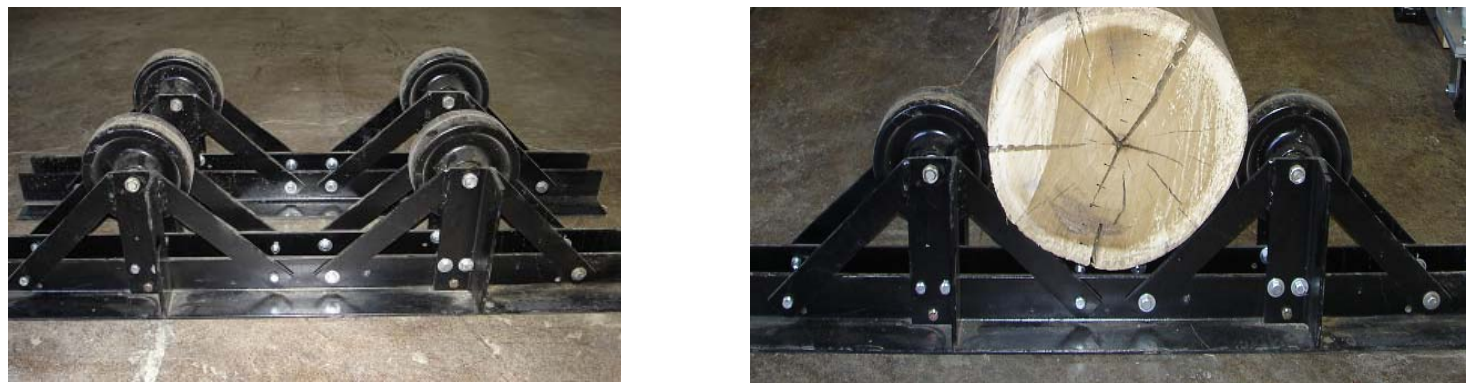

Figure 3-6 Rollers for supporting and rotating logs 


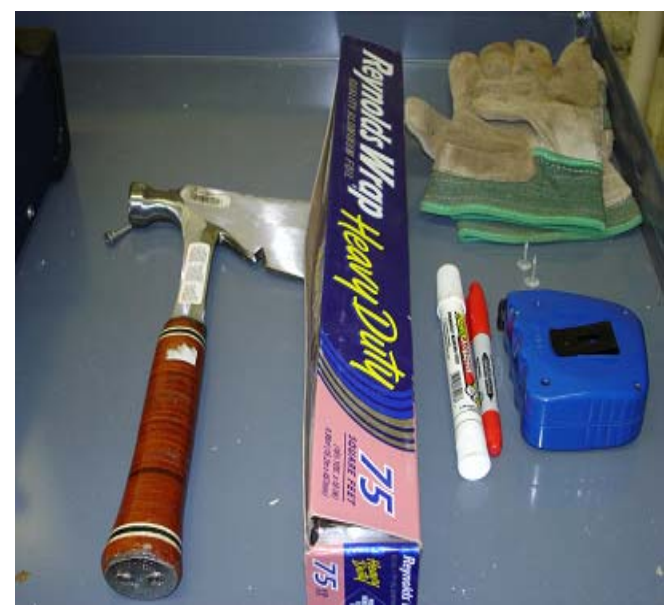

(a)

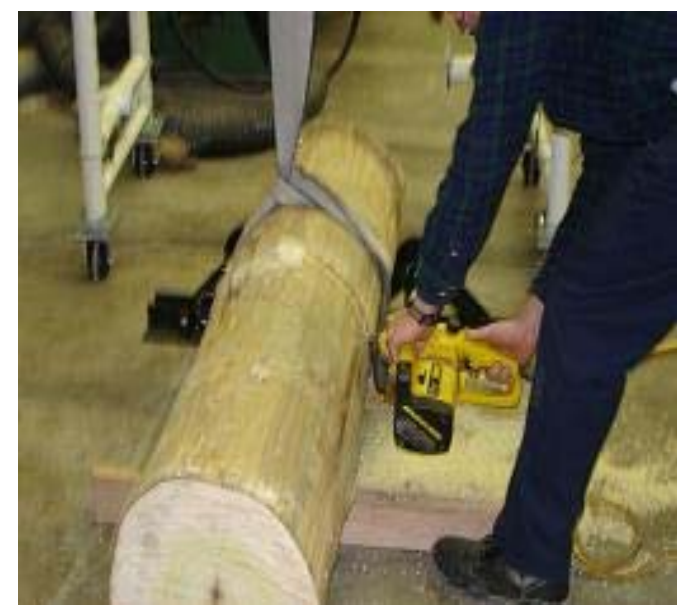

(b)

Figure 3-7 (a) Axe, aluminum foils, gloves, measuring tape, and markers, (b) an 18 in saw being used for cutting logs to check for defects

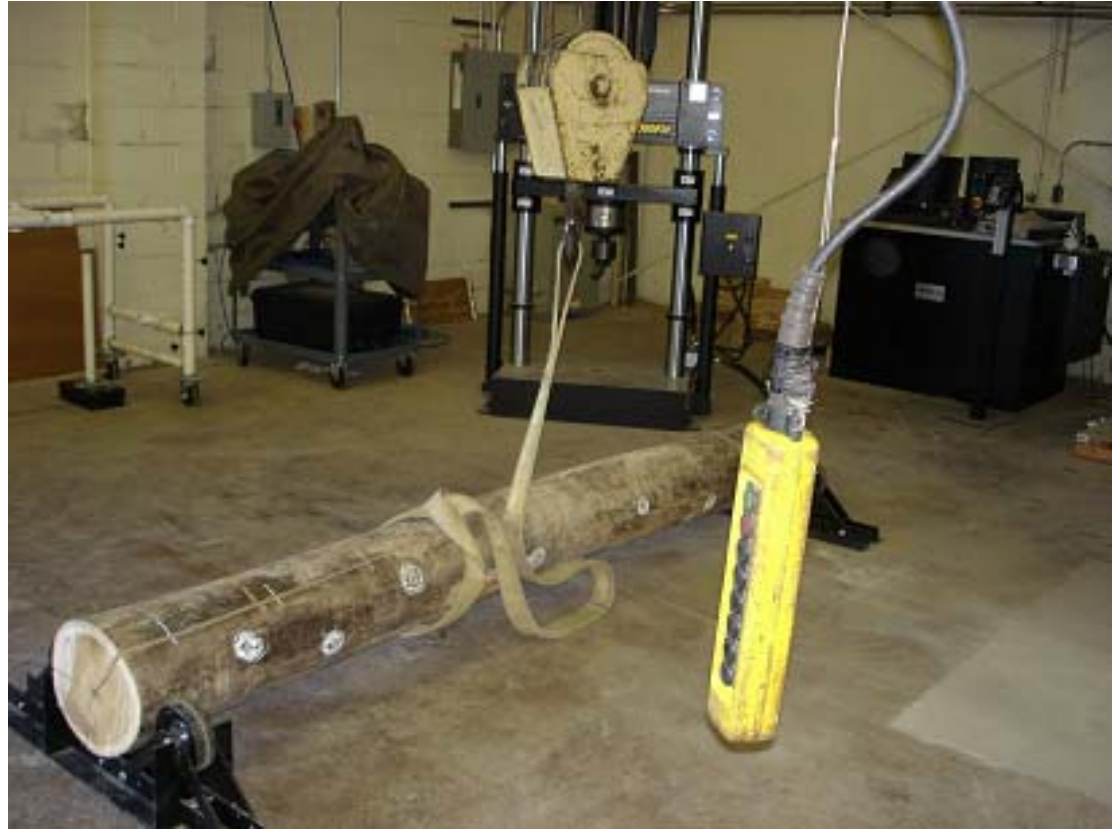

Figure 3-8 Showing the crane and the control switches for lifting the logs 
Table 3-1 System Specification (Loulizi 2001, GSSI 2005)

\begin{tabular}{|c|c|}
\hline \multicolumn{2}{|c|}{ SIR-20 GPR System Parameters } \\
\hline Antenna Compatibility & $\begin{array}{l}\text { Operates with any GSSI model antenna and can handle up to two antenna } \\
\text { simultaneously }\end{array}$ \\
\hline Sampling frequency rate & Up to $100 \mathrm{kHz}$ \\
\hline Output Data Format & 8- or 16-bit, selectable \\
\hline $\begin{array}{l}\text { Number of samples per } \\
\text { second }\end{array}$ & $256,512,1024,2048$, selectable \\
\hline Scan rate & 2 to 800 scans/second, selectable (now reduced due to FCC regulations) \\
\hline Resolution & 5 picoseconds \\
\hline $\begin{array}{l}\text { Range (Programmable } \\
\text { sampling window } \\
\text { increments) }\end{array}$ & $0-8,000$ nanoseconds full scale, user selectable \\
\hline Dynamic range & $>110 \mathrm{~dB}$ \\
\hline Signal to noise ratio & $>100 \mathrm{~dB}$ \\
\hline Time base accuracy & $.02 \%$ \\
\hline $\begin{array}{l}\text { Programmable stacking } \\
\text { range }\end{array}$ & 2 to 32768 scans \\
\hline \multicolumn{2}{|l|}{ Acquisition Software } \\
\hline Antennas & Records data from 1 or 2 channels simultaneously \\
\hline Display modes & $\begin{array}{l}\text { Linescan, Wiggle Plot and Oscilloscope. } \\
\text { In linescan display, } 256 \text { color bins are used to represent the amplitude and } \\
\text { polarity of the signal }\end{array}$ \\
\hline Automatic system setups & $\begin{array}{l}\text { Storage of an unlimited number of system setup files for different road } \\
\text { types, survey conditions, and/or antenna deployment configurations }\end{array}$ \\
\hline Range gain & $\begin{array}{l}\text { Manual adjustment from }-20 \text { to }+100 \mathrm{~dB} \text {. Number of segments in gain } \\
\text { curve is user selectable from } 1 \text { to } 8\end{array}$ \\
\hline Vertical filters & $\begin{array}{l}\text { Individually filter the scans in the time domain. Low and high Pass, Infinite } \\
\text { Impulse Response (IIR), Finite Impulse Response (FIR), Boxcar and } \\
\text { Triangular filter types are available } \\
\text { IIR } \\
\text { Low Pass } 2 \text { poles } \\
\text { High Pass } 2 \text { poles } \\
\text { FIR, Boxcar and Triangle } \\
\text { Low Pass up to } 1 / 2 \text { scan length } \\
\text { High Pass up to } 1 \frac{1}{2} \text { scan length }\end{array}$ \\
\hline Horizontal filters & $\begin{array}{l}\text { IIR } \\
\text { Stacking } 1 \text { to } 16384 \text { scans } \\
\text { Background Removal } 1 \text { to } 16384 \text { scans } \\
\text { Static } \\
\text { Stacking } 2 \text { to } 32768 \text { scans } \\
\text { Background Removal } \\
\end{array}$ \\
\hline \multicolumn{2}{|l|}{ Antenna } \\
\hline $\begin{array}{l}\text { Ground-coupled } \\
\text { Center frequency } \\
\text { Pulse width }\end{array}$ & $\begin{array}{l}\text { Model 3101D } \\
900 \mathrm{MHz} \\
1.1 \mathrm{~ns}\end{array}$ \\
\hline
\end{tabular}


Table 3-2 Range of antennas manufactured by GSSI and its applications (GSSI 2005)

\begin{tabular}{|c|c|c|c|}
\hline Model & Center Frequency & $\begin{array}{c}\text { Depth } \\
\text { of Penetration }\end{array}$ & Typical Applications \\
\hline \multicolumn{4}{|c|}{ Ground-Coupled Antennas } \\
\hline 5100 & $1500 \mathrm{MHz}$ & $.5 \mathrm{~m}$ & Concrete Evaluation \\
\hline 3101D & $900 \mathrm{MHz}$ & $1 \mathrm{~m}$ & $\begin{array}{l}\text { Concrete Evaluation, Void } \\
\text { Detection }\end{array}$ \\
\hline 5103 & $400 \mathrm{MHz}$ & $4 \mathrm{~m}$ & $\begin{array}{l}\text { Utility, Engineering, } \\
\text { Environmental, Void Detection }\end{array}$ \\
\hline 5104 & $270 \mathrm{MHz}$ & $6 \mathrm{~m}$ & Utility, Engineering, Geotechnical \\
\hline 5106 & $200 \mathrm{MHz}$ & $7 \mathrm{~m}$ & $\begin{array}{l}\text { Geotechnical, Engineering, } \\
\text { Environmental }\end{array}$ \\
\hline 3207 & $100 \mathrm{MHz}$ & $20 \mathrm{~m}$ & $\begin{array}{l}\text { Geotechnical, Environmental, } \\
\text { Mining }\end{array}$ \\
\hline $3200 \mathrm{MLF}$ & $16-80 \mathrm{MHz}$ & $25-35 m$ & Geotechnical \\
\hline \multicolumn{4}{|c|}{ Air-Launched Antennas } \\
\hline 4105 Horn & $2.2 \mathrm{GHz}$ & $\begin{array}{l}\text { up to } 0.75 \mathrm{~m} \\
\text { depending on } \\
\text { medium }\end{array}$ & $\begin{array}{l}\text { Pavement Thickness and Road } \\
\text { Condition Assessment }\end{array}$ \\
\hline 4108 Horn & $1000 \mathrm{MHz}$ & $1 \mathrm{~m}$ & $\begin{array}{l}\text { Highway and Bridge Deck } \\
\text { Evaluations }\end{array}$ \\
\hline \multicolumn{4}{|c|}{ Borehole Antennas } \\
\hline $\begin{array}{l}\mathrm{TW}-100 \\
\mathrm{Tx} / \mathrm{Rx}\end{array}$ & $100 \mathrm{MHz}$ & $20 \mathrm{~m}$ & \multirow{3}{*}{$\begin{array}{l}\text { For Geophysical Applications, } \\
\text { Foundation Inspections, and } \\
\text { Evaluation of Subsurface Pipes } \\
\text { and Utilities }\end{array}$} \\
\hline TW-100 Tx & & & \\
\hline $\begin{array}{l}\text { TW-1000 } \\
\text { Tx/Rx }\end{array}$ & $1000 \mathrm{MHz}$ & $1 \mathrm{~m}$ & \\
\hline
\end{tabular}




\section{Chapter 4 \\ DATA ACQUISITION AND INTERPRETATION PROCEDURE}

In order to identify the subsurface defects in a log and evaluate its condition, the ground penetrating radar (GPR) data has to be collected and processed, and the anomalies in the GPR scan have to be identified. The extraction of physical conditions occurs in three steps, which are, data collection, data processing, and display and review data for defect detection. The process of data acquisition and interpretation is explained next.

\subsection{DATA ACQUISITION}

Data was collected using GSSI SIR-20 system. The antenna used in this research was ground coupled dipole antenna with central frequency of $900 \mathrm{MHz}$. The specifications of the SIR-20 system and the antenna have been discussed in the previous chapter. Data collection involved log preparation, selection of data collection parameters, system initialization, and scanning of the log to collect GPR data.

Before starting the experiments, distance between the rollers were adjusted to accommodate the full length of the log to be scanned. Projecting knots were chipped off using an axe to move the antenna closer to the log surface without interference from the projecting knots. Characteristics of the log such as species, length, diameter, and moisture content at different locations (surface to center of the $\log$ ) were noted. A sample GPR scan was taken to calculate the dielectric constant of the log. The log was marked with section numbers for GPR scanning and sawing purpose as shown in Figure 4-1. If one end of the log had a smaller diameter compared to the other end (tapered log), the smaller end had to be lifted up and/or adjusted horizontally using wooden blocks as shown in Figure 4-2. This ensured that the distance of the antenna from the log surface was more or less constant and within 0.5 " to $1 "(12-25 \mathrm{~mm})$.

Upon completion of log preparation, the specimen was scanned by moving the antenna frame in the longitudinal direction (along the length of the log). Additional GPR scans were taken by rotating the log. The number of longitudinal scans per log depended upon the diameter of the log. The input parameters varied from $\log$ to $\log$. The various input parameters are discussed in the following paragraphs. 


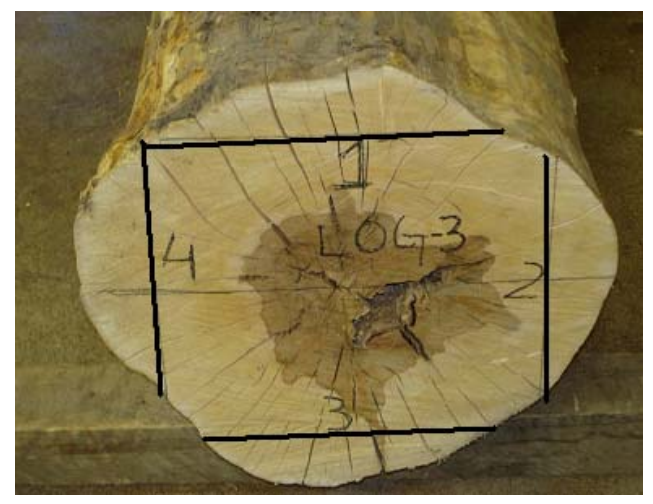

Figure 4-1 Lines marked on Log 3 for GPR scanning and sawing purpose

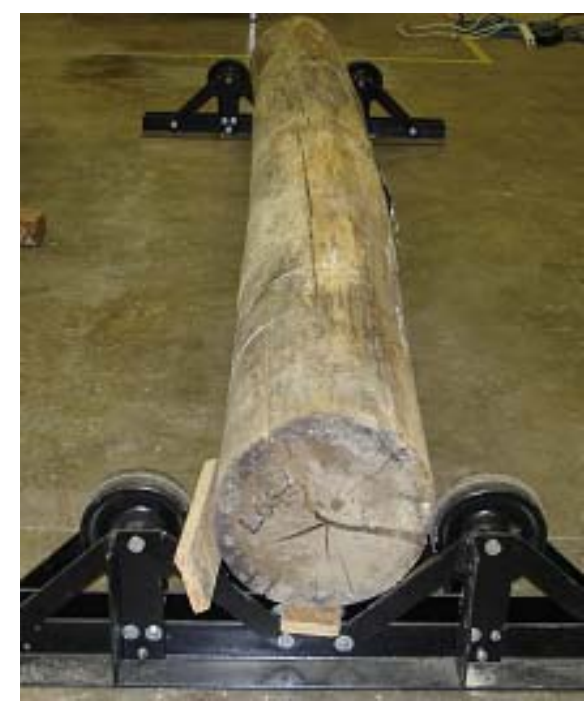

Figure 4-2 Picture showing wooden pieces under the small end of the $\log 4$ to raise the level

The software parameters were maintained the same for all the GPR scans in order to maintain a level of consistency. Linear units were set to meters (SI Unit) and the temporary database marker (showing distance marks) option was selected. After the software parameters were set, a new project was created. Data collection mode was set to "survey wheel" to identify the location of the defects with greater accuracy. When set to survey wheel mode, data is collected based on the rotation of the survey wheel with number of scans per meter set by the individual personnel. Depending on the diameter of the survey wheel and the optical encoder used, a calibration parameter is given as input, which is used by the system to convert wheel rotation to distance traveled. For the Model 
611 survey wheel used in this research, the calibration parameter was set to 609.6 ticks/ft or 2000 ticks/m (GSSI 2002a).

The various data collection settings and parameters are shown in a header file in Figure 4-3. It is very important to first set the antenna configuration name to the correct channel, namely channel 1, channel 2, or multi-channel (using both channels). The data collection parameters including scans/second, scans/meter, and meter/mark (marker setting) were user-specified inputs that respectively affected how many scans of GPR data are collected in a second, how many scans are collected based on distance traveled, and how many visual marks will be placed at a specified distance. Other user defined parameters include time range (ns), samples/scan and bits/sample that affect the sample depth and resolution of the data, and also the signal quality. The other parameters that had to be specified were dielectric constant, antenna transmit rate, and configuration (Comp) of the transmitter and receiver antennas. Typical values for the data collection parameters for this study are shown in Figure 4-3. However, slightly different values of the parameters such as scans/sec and dielectric constant were used for each log as explained later in chapter 5. The Top and Depth parameters shown in Figure 4-3 are processing parameters used to specify the top location and depth of the full GPR scan, if known. These parameters were not used in this study since the computation of the dielectric constant usually results in more accurate depth estimations.

As shown in Figure 4-3, the survey wheel was set to record data at a distancebased rate of 472.441 scans per meter which amounts to 12 scans/inch in the final GPR scan. The quality of this GPR scan is affected by other scanning parameters. The SIR-20 data acquisition system can collect data at rates of up to $153 \mathrm{scans} / \mathrm{second}$. Also, if the antenna transmit rate is set to a high number ( $\sim 75$ to $100 \mathrm{kHz}$ ) the unit provides optimum data quality as a result of more sample-averaging and increased signal to noise ratio. However, it is important to simultaneously reduce the scan rate below the 153 scans/second to increase sample-averaging. Reducing the scans/second also requires reducing the speed at which the data collection frame is moved over the log. Therefore, to optimize the performance of SIR-20 in terms of speed and quality of signal, system generated scans were set to the rate between 70 and 100 scans/second. 


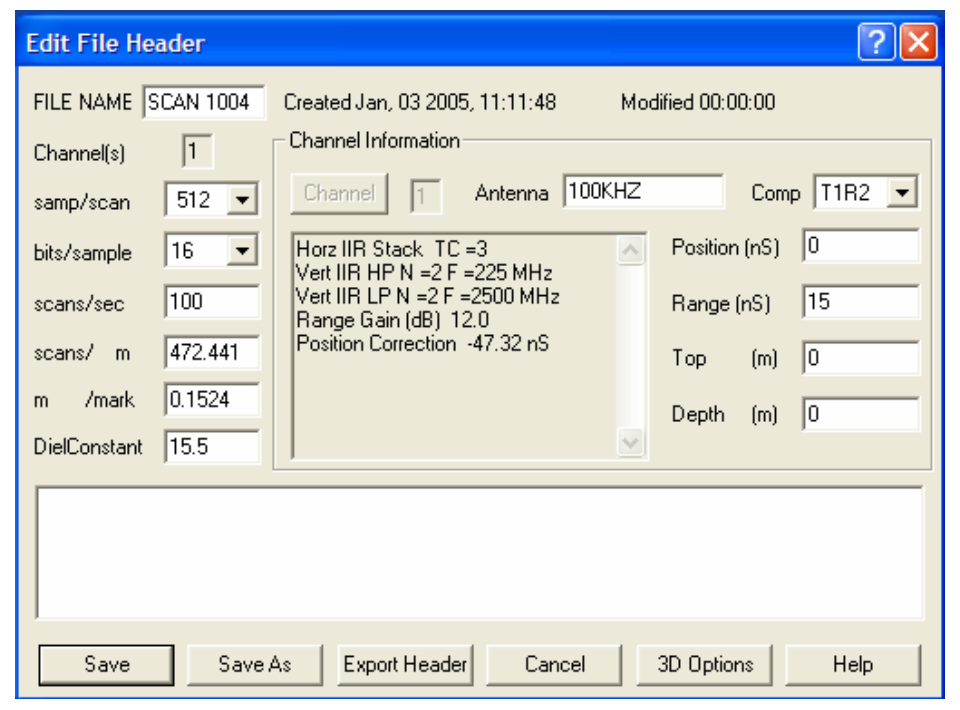

Figure 4-3 Data collection parameters

After the data collection parameters were entered, a "macro" was attached. A macro is a set of saved parameters that the system applies to the entire dataset during data collection. It is a list of preset parameters that tells the mainframe how to collect data. These parameters include: position correction, range gain, and Infinite Impulse Response (IIR) filters (GSSI 2002a, GSSI 2002b). Different frequency antennas have different set of macro parameters specified by the system, and the software provides the user with a set of preloaded macros. The user has to select the appropriate macro corresponding to the antenna frequency used for the experiments.

After attaching the appropriate macro, system initialization was conducted while placing the antenna close to the surface of the $\log (<12-25 \mathrm{~mm}$ distance $)$. Upon the first initialization of the antenna, preliminary scan of the log enables the user to check and manually adjust the range. If the range is less than what is needed to reach the bottom it should be increased. If the range is too high, it should be reduced. It is important to note that a reduced range results in higher resolution, that is, higher data quality. Therefore, the range should was set close to a minimum possible value. During data acquisition, the auto gain was turned off in order to preserve the original signal. Instead, a user-specified constant gain was applied over the entire depth to the whole data for easy detection of defects. Once this gain was set, the system was ready for actual scanning of the log. Figure 4-4(a) shows scanning of a log with antenna on the top and Figure 4-4(b) shows scanning the log from the side. 


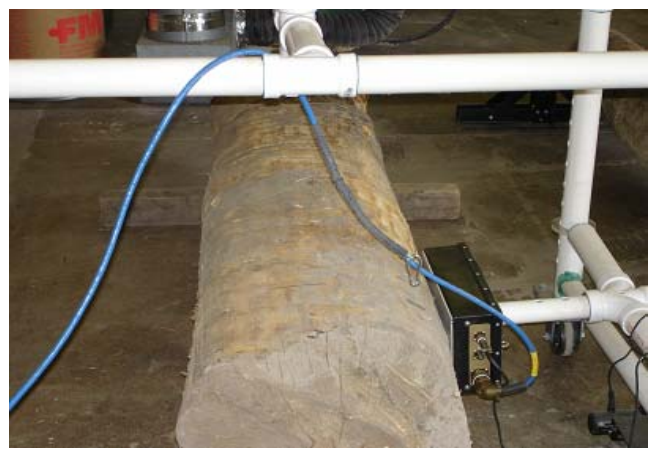

(a)

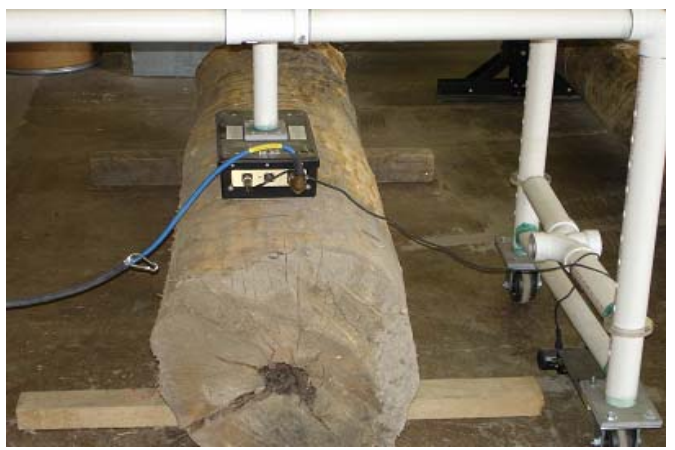

(b)

Figure 4-4 Antenna configuration (a) antenna on top (b) antenna on side

Prior to scanning, the specimen was marked at the point of start of scan with white paint to indicate the starting point. Also, aluminum foil was placed on the opposite face of the $\log$ (Figure 4-5) to clearly identify the bottom of the log, which is necessary to compute an average value for the dielectric constant. In some logs, the bottom was visible in the GPR scan even without the use of aluminum foil, which then became wood-air interface and resulted in reverse polarity echo compared to the top surface echo in the GPR scan.

Each scanning commenced from the butt end (i.e., end with larger diameter) of the log, and the antenna deployment frame was moved along the length of the specimens (i.e., a longitudinal scan). During the entire scanning, the gap between the log and the antenna surface was maintained at less than $25 \mathrm{~mm}$. After one scan was completed, the $\log$ was rotated and the data collection process was repeated with the exact same data collection settings and parameters. The system initialization process had to be repeated at the beginning of each scan. Depending on the diameter of the log, longitudinal GPR scans were conducted for four to ten rotational positions around the log.

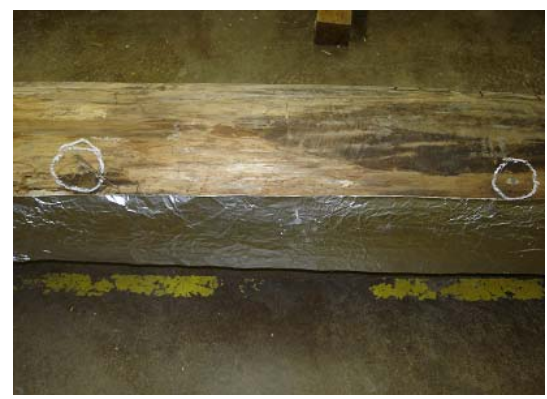

Figure 4-5 Aluminum foil at the opposite face of the log 
The effect of curvature was a concern for the experiments because the antenna is flat and the logs are curved, thus creating a non-uniform air gap or surface coupling over the width of the antenna. Higher curvature (smaller diameter logs) usually resulted in worse signal to noise ratio. By maintaining a consistent air-gap between the antenna and the log during scanning, it is possible to filter out the relatively constant noise due to curvature and enhance the data quality during post-processing.

The collected data sets were viewed using RADAN 5.0 software (GSSI 2002b). Figure 4-6 shows the raw GPR data collected using SIR-20 system. The raw data usually provides very little detail of the log, and post-processing is needed to provide a clear picture of the subsurface defects.

\subsection{DATA PROCESSING}

Post-processing of the acquired data was necessary to enhance the features (signal echos) in radar data scan and reduce the noise. This greatly helps in detecting the subsurface defects and identifying the bottom of the log. In the raw data (Figure 4-6), set of black and white strips near the top of the scan indicates the first reflection from the interface between the antenna and the log surface. Additional signal clutters (localized black and white strips) occur near the anomalies resulting from internal defects, embedded metals, or changes in wood density. Some times the signal clutters are too weak and need some amplification for them to become visible in the processed scan.

In this research, RADAN 5.0 software was used for data processing. Figure 4-6 shows raw GPR data that will be used for explaining the data processing procedure. As shown in this figure, the display options allow the vertical scale to be set to sample, time (ns), or depth, and the horizontal scale can be set to distance (or scans) along the surface of the log. Basic data post-processing involves some fundamental manipulation of raw data to enhance the data for easier data interpretation. The basic steps involved in postprocessing are applying range gain, zero correction, background removal and noise filtering. Range gain and zero correction can be interchanged to be the first processing step. All these steps are explained in detail in the following paragraphs. 


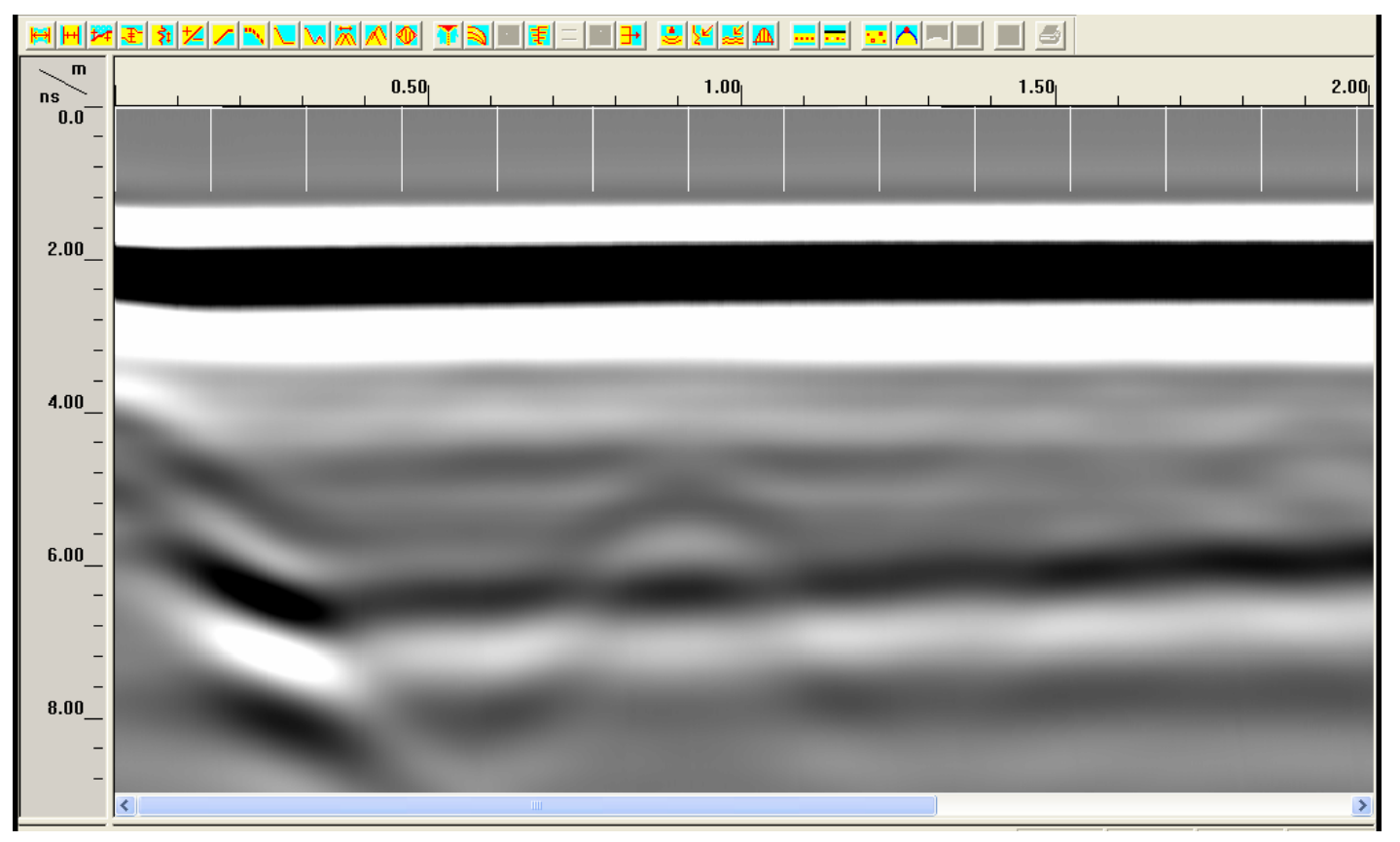

(a)

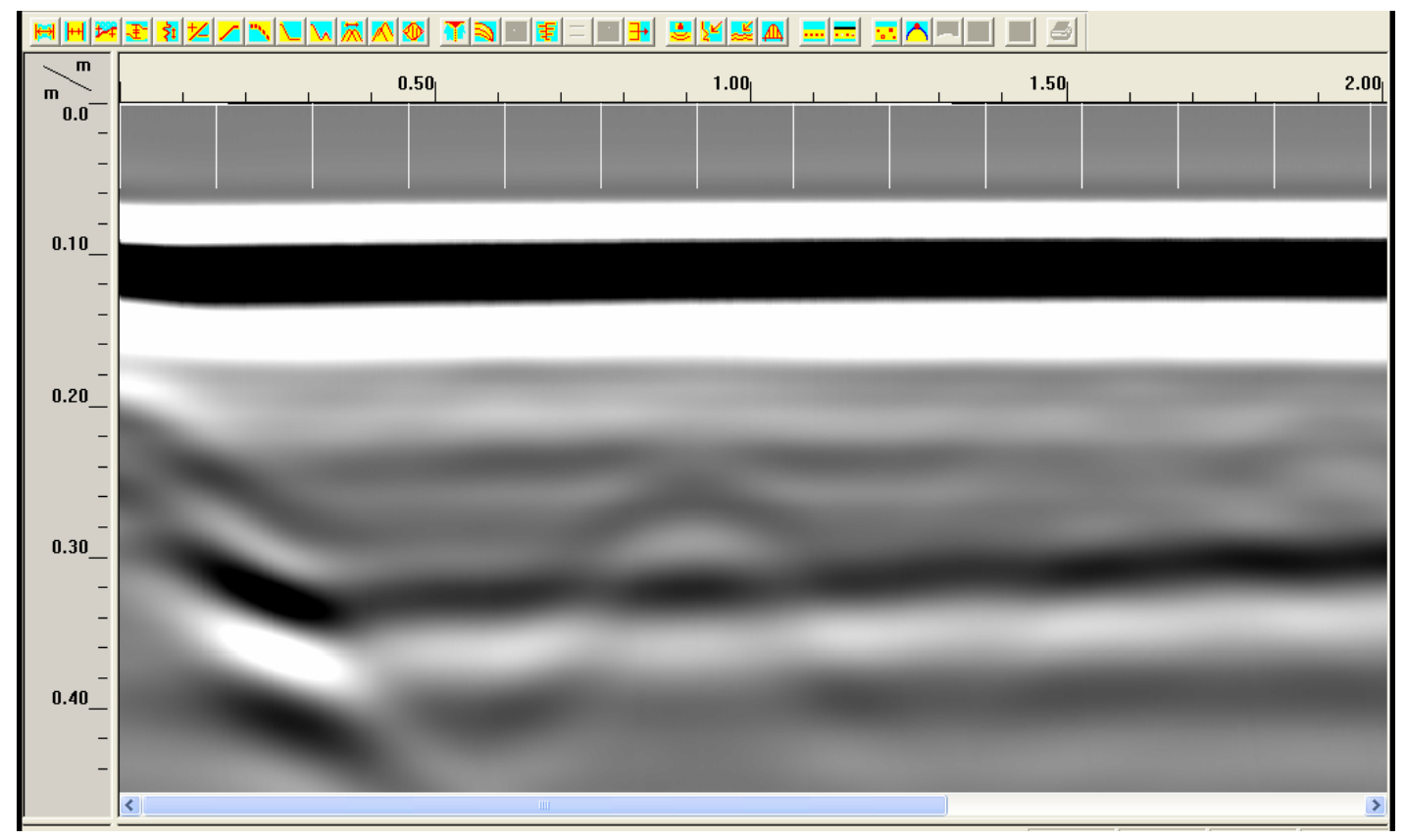

(b) 


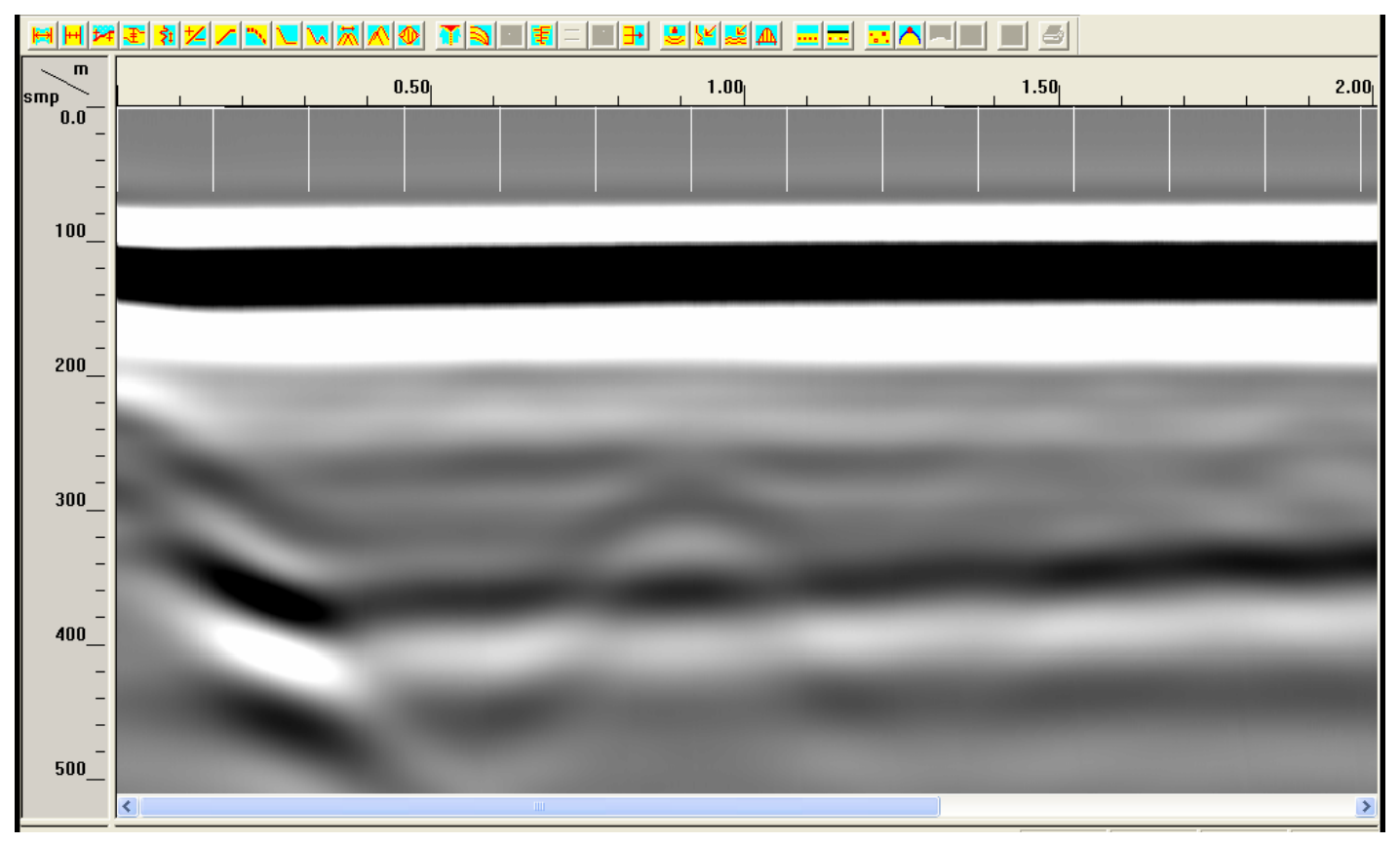

(c)

Figure 4-6 Raw GPR data with vertical scale indicating (a) time (ns) (b) depth (m) (c) sampling points, and the horizontal scale indicating the distance $(\mathrm{m})$ along the log

\subsubsection{Range Gain}

Radar signals are prone to attenuation with increasing depth. Because of attenuation, the information at greater depths is not as clear and as reliable as the data near the surface of the antenna. RADAN software allows increasing time (or depth) dependent gain which compensates for amplitude reduction with depth. One of the major problems is that the variation of attenuation with depth is not uniform. In general, the attenuation of radar signals is low near the surface but very high towards the center of the logs due to high moisture content in the center.

RADAN provides three different types of gains: Automatic Gain, Linear Gain, and Exponential Gain. A typical radar signal (extracted from the scan in Figure 4-6) is shown in Figure 4-7. Linear function applies a linear gain between gain nodes (along the depth) where as exponential function applies an exponential gain curve. For linear and exponential gains, the user can select up to 16 nodes over the depth and define the gain at each nodal point. Linear and exponential gains are used to amplify all the scans in the data set. On the other hand, the automatic gain feature (which applies a constant gain over the entire depth for any given waveform) attempts to balance the gain for each scan 
(GSSI, 2002b). Figure 4-8 shows comparison of the signals after applying different types of gains to the signal shown in Figure 4-7. In this research, a linear gain was applied to the raw GPR data from the logs since it provided significant gain for deeper and more attenuated echoes. Figure 4-9 shows a typical GPR file after applying a linear gain varying from 0 to 5 over the entire depth and a temporary gain of 16 (for display only). The second echo from the bottom of the log and some of the internal features become more obvious after the gain was applied. These echoes are not clearly visible in the raw data file shown in Figure 4-6.

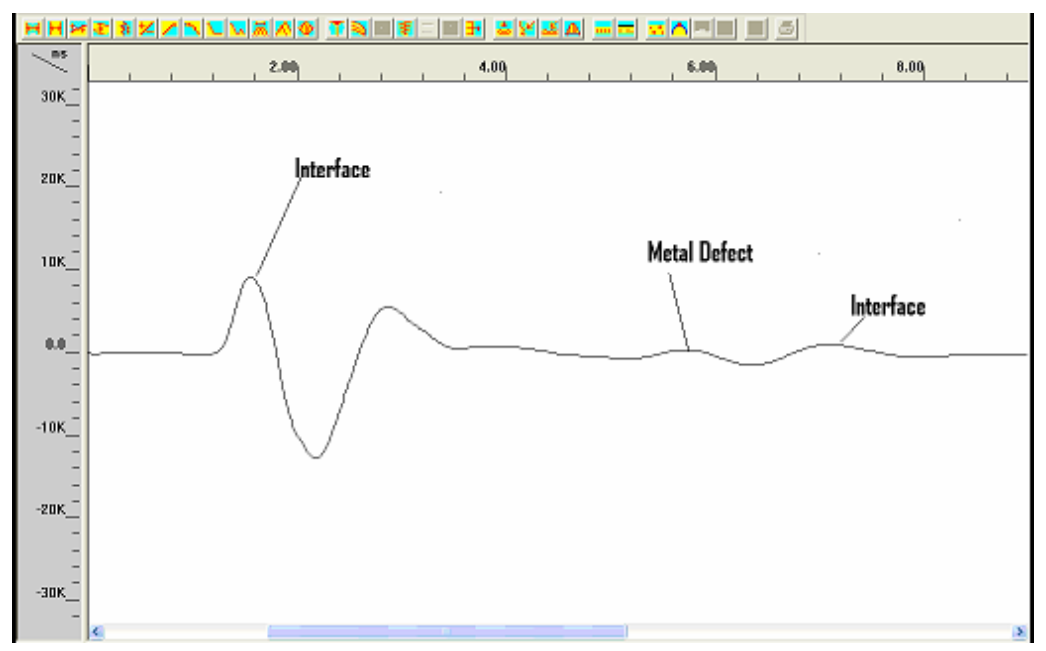

Figure 4-7 Typical radar signal observed using GPR oscilloscope mode

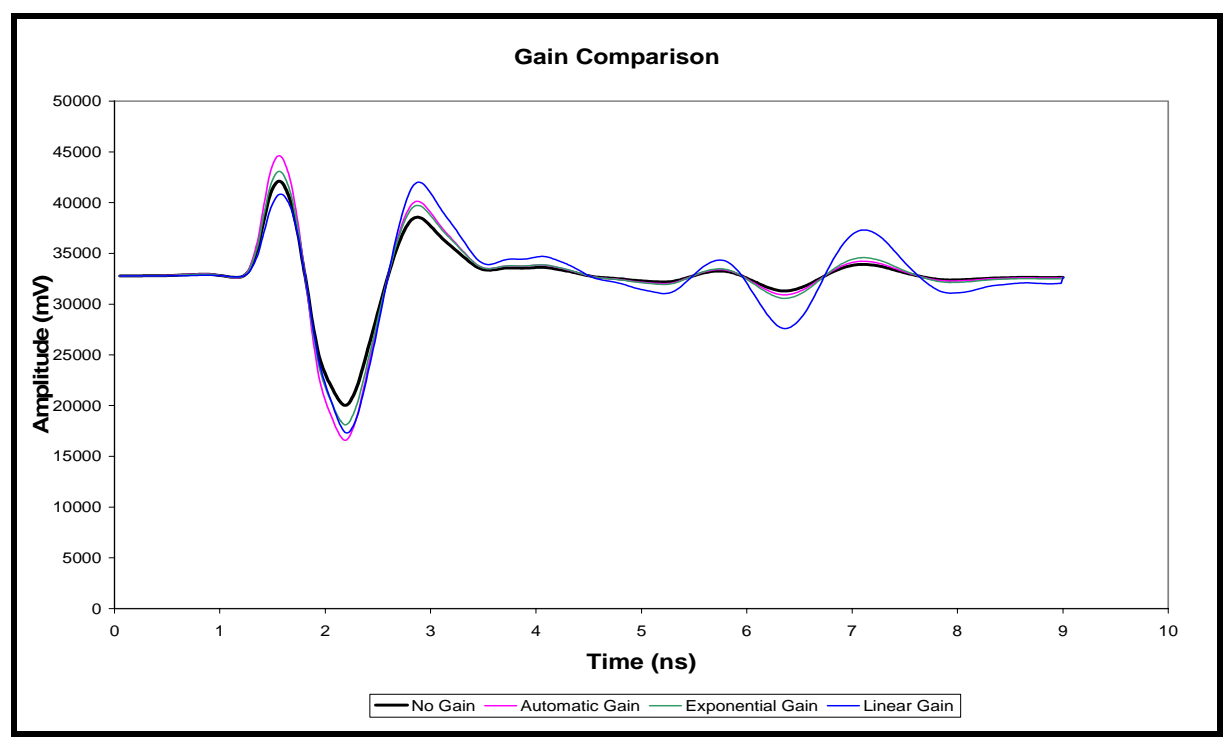

Figure 4-8 Signal comparison for different types of applied gains 


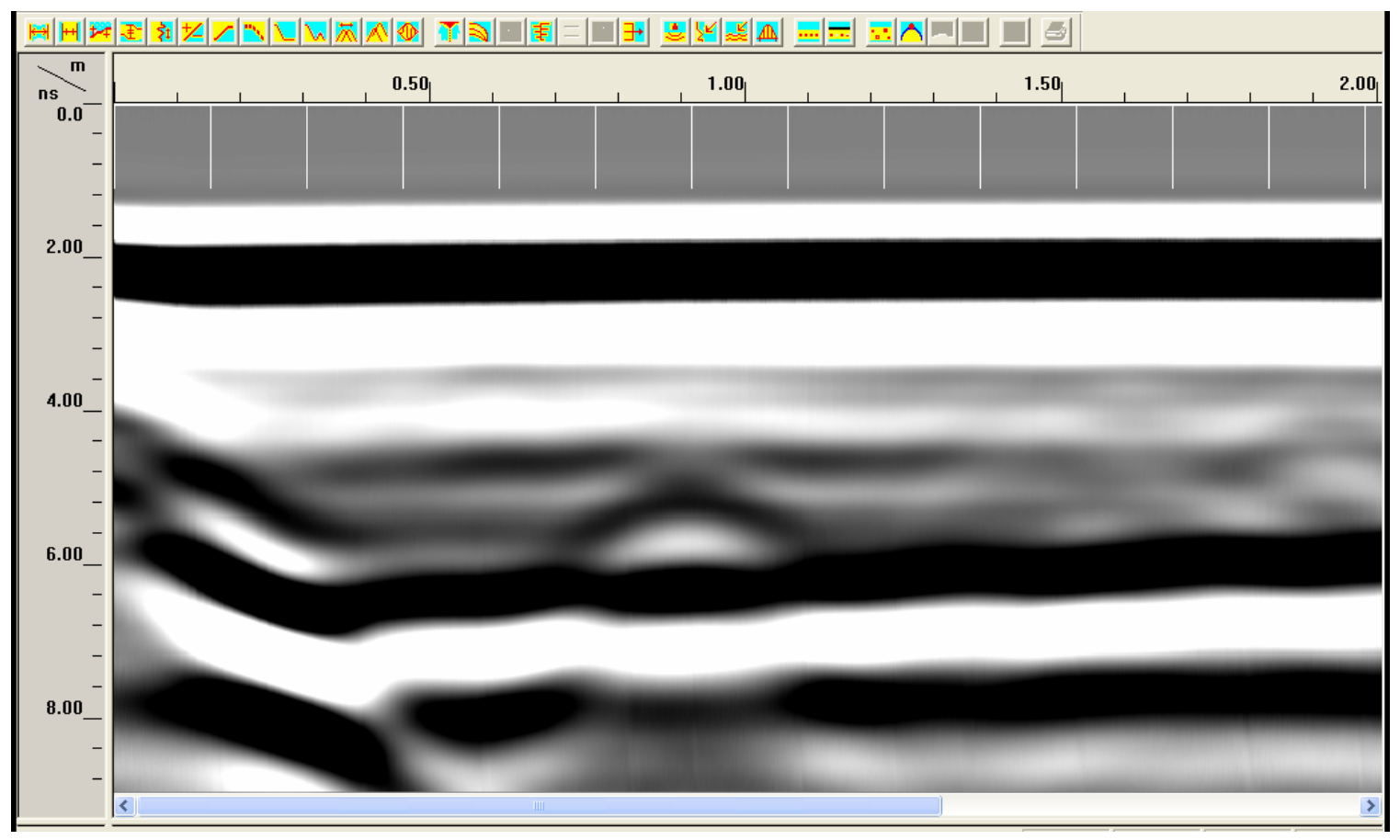

(a)

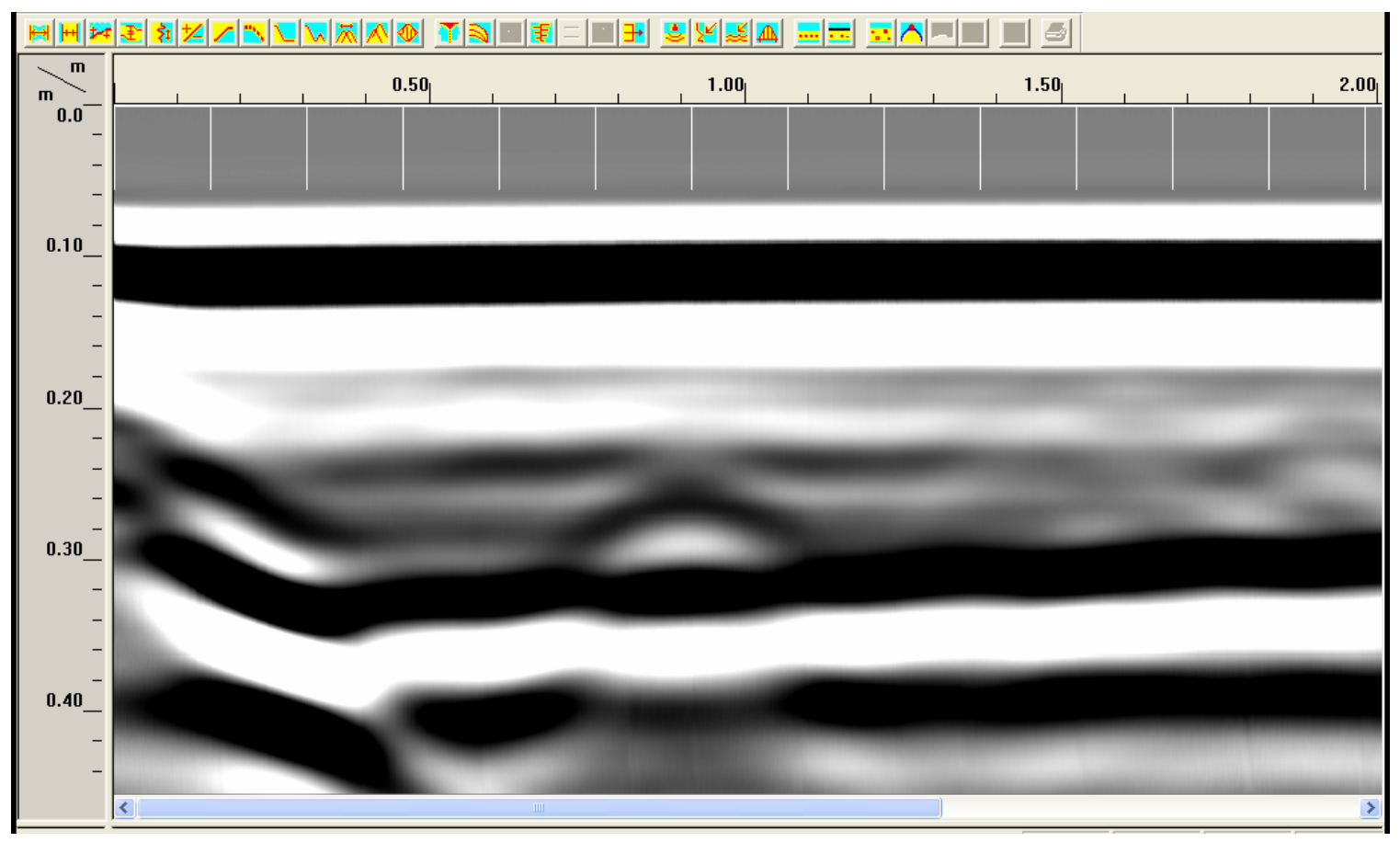

(b)

Figure 4-9 Typical GPR file of a log (a) distance vs. time (ns) (b) distance vs. depth (m) 


\subsubsection{Zero Correction}

Zero correction is a process that is used to vertically adjust the position of the whole GPR scan in the data window so that the depth can be measured with respect to the ground surface. This correction involves shifting the first positive peak of the direct wave from the antenna (i.e., reflection from the antenna-log interface) in such a way that it becomes centered at the top edge of the data window, which then corresponds to the ground surface. After the zero correction is applied, the "Position" parameter in the file header has to be changed to a value of zero. This process allows estimation of the correct depth of any observed feature after the dielectric correction is made. Figures 4-10 shows the GPR data after applying zero correction. The Figures in 4-10 (a) and 4-10(b) show the vertical axis in terms of time (ns) and depth, respectively. While these figures display several GPR waveforms along the length of the log as a grey scale intensity scan as explained earlier in Chapter 2, it is possible to view each individual signal in the oscilloscope mode as shown in Figure 4-11.

To read the two-way travel time or depth from Figure 4-10 or 4-11, it is important to note that the reflection from the bottom of the log (wood-air interface) has opposite phase compared to the reflection from antenna-wood interface (top of the log). The top interface in Figure 4-10(c) appears as white-black-white band, with the center of the first white band taken as the origin for depth measurement. The corresponding point in the bottom interface for reading the two-way travel time (Figure 4-10(a)) or depth (Figures 410(b) and 4-10(c)) is the center of the first black band in the black-white-black band corresponding to the bottom reflection. It should be noted that the characteristic of the bottom reflection is often altered due to the reflection echoes from subsurface defect close to the bottom, and this may influence the presence of white or black bands. In general, reflections from subsurface defects like knots and embedded metals have the same phase as the top reflection as shown in Figure 4-11. It is generally easier to read the actual two-way travel time and depth corresponding to the subsurface defects and bottom of the log from the oscilloscope signals as shown in Figure 4-11(a) and 4-11(b), which show the defect reflection as positive peak and bottom reflection as negative peak. 


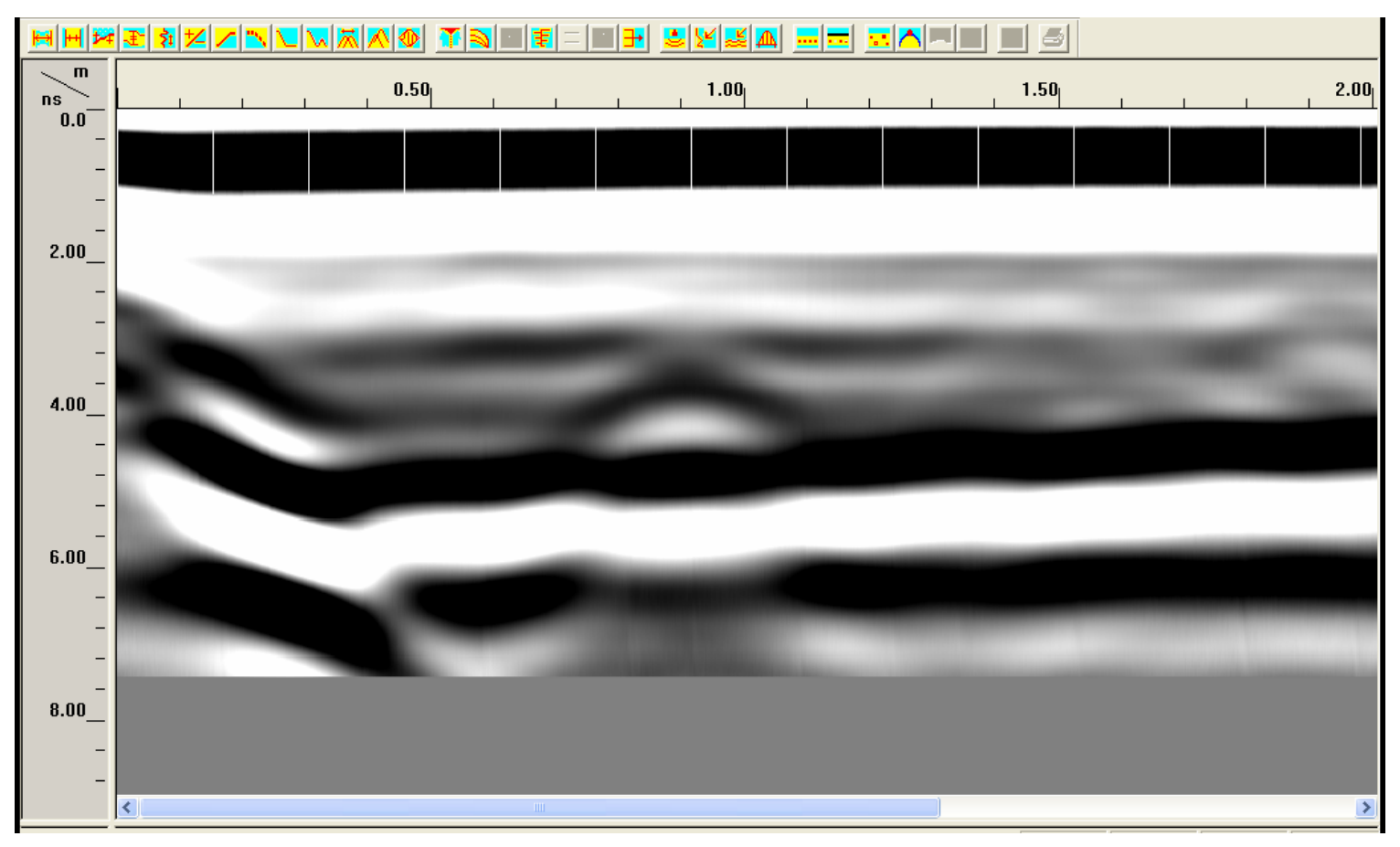

(a)

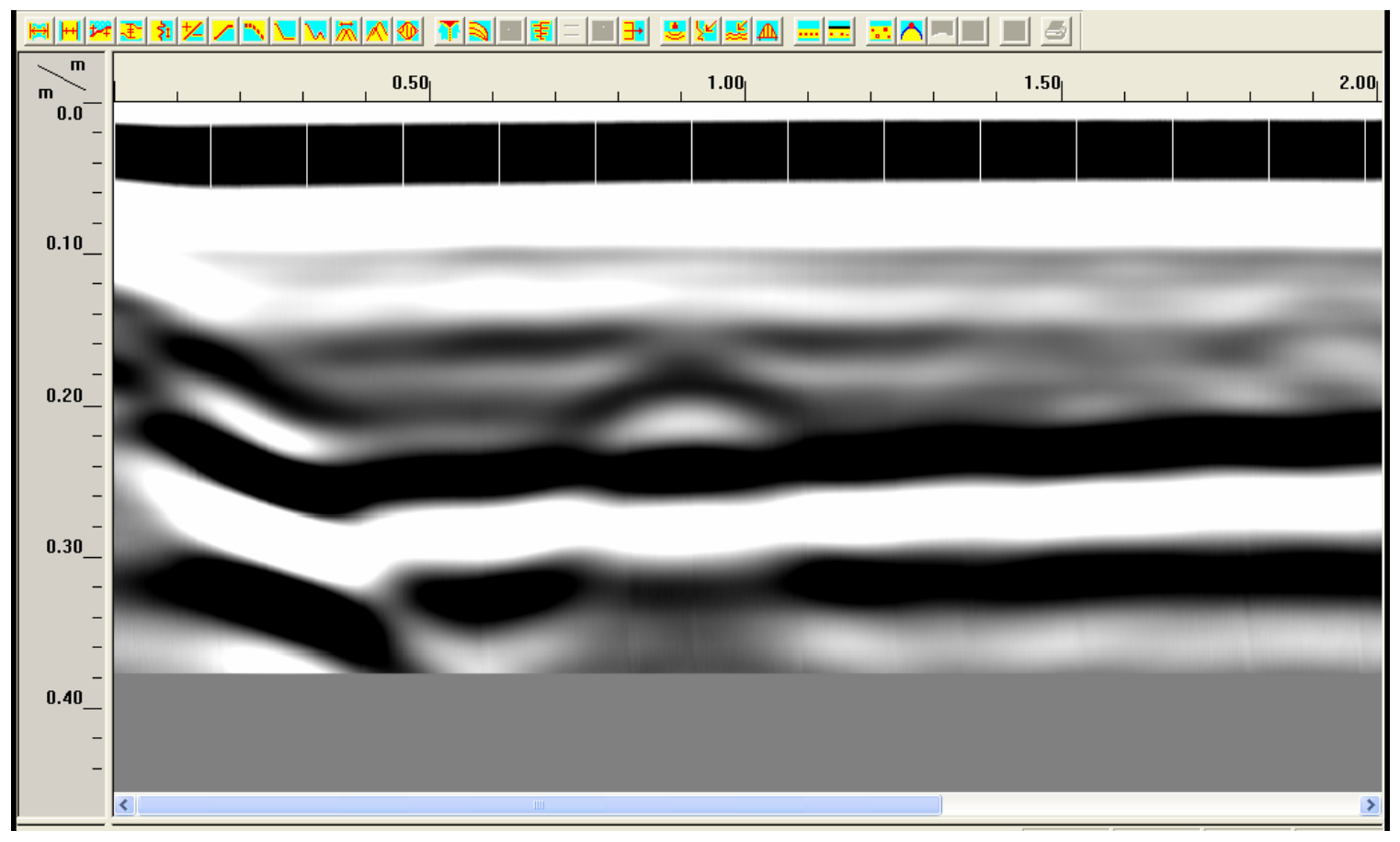

(b) 


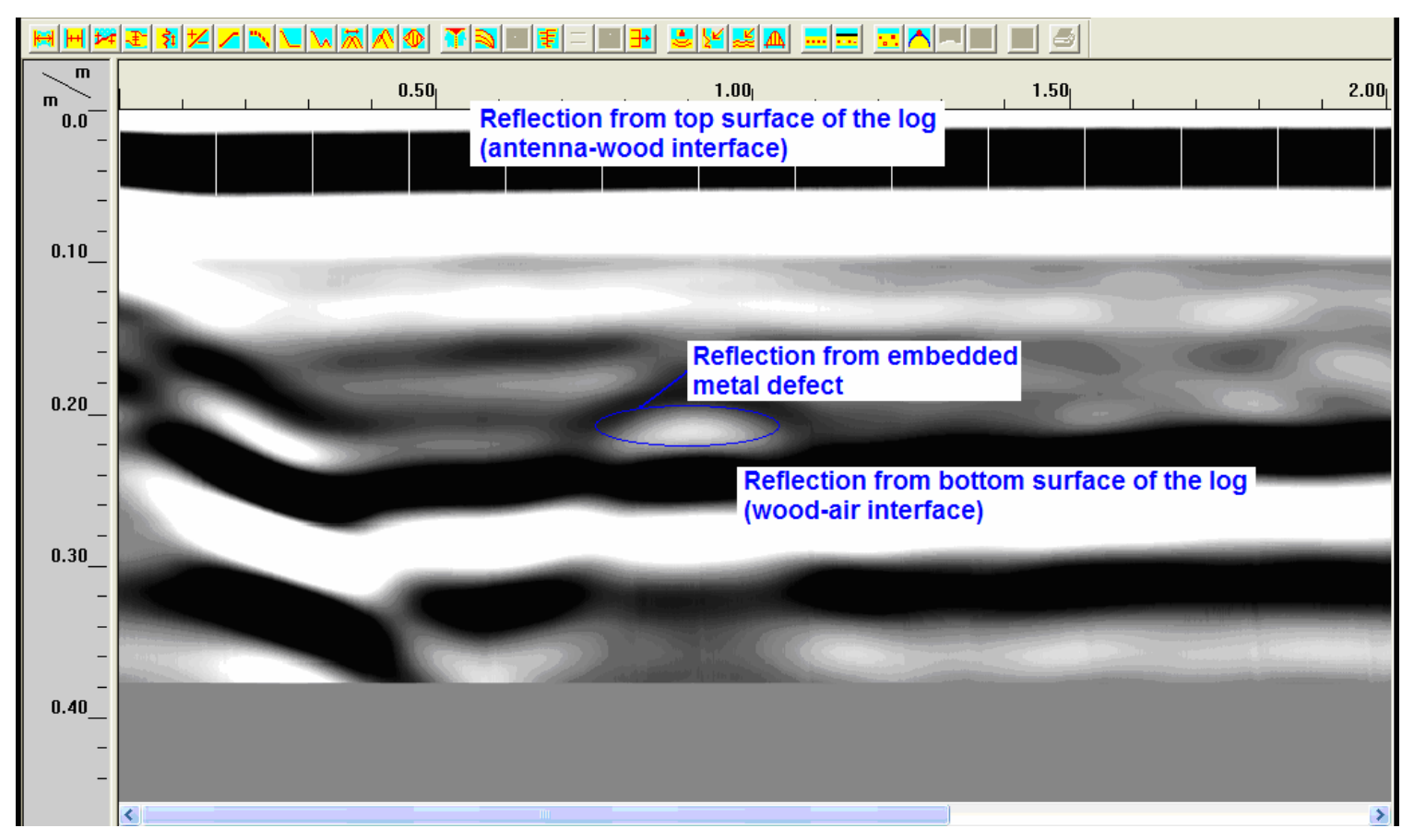

(c)

Figure 4-10 GPR data after applying zero correction (a) time (ns) plot (b) depth (m) plot (c) depth (m) plot showing the various reflections

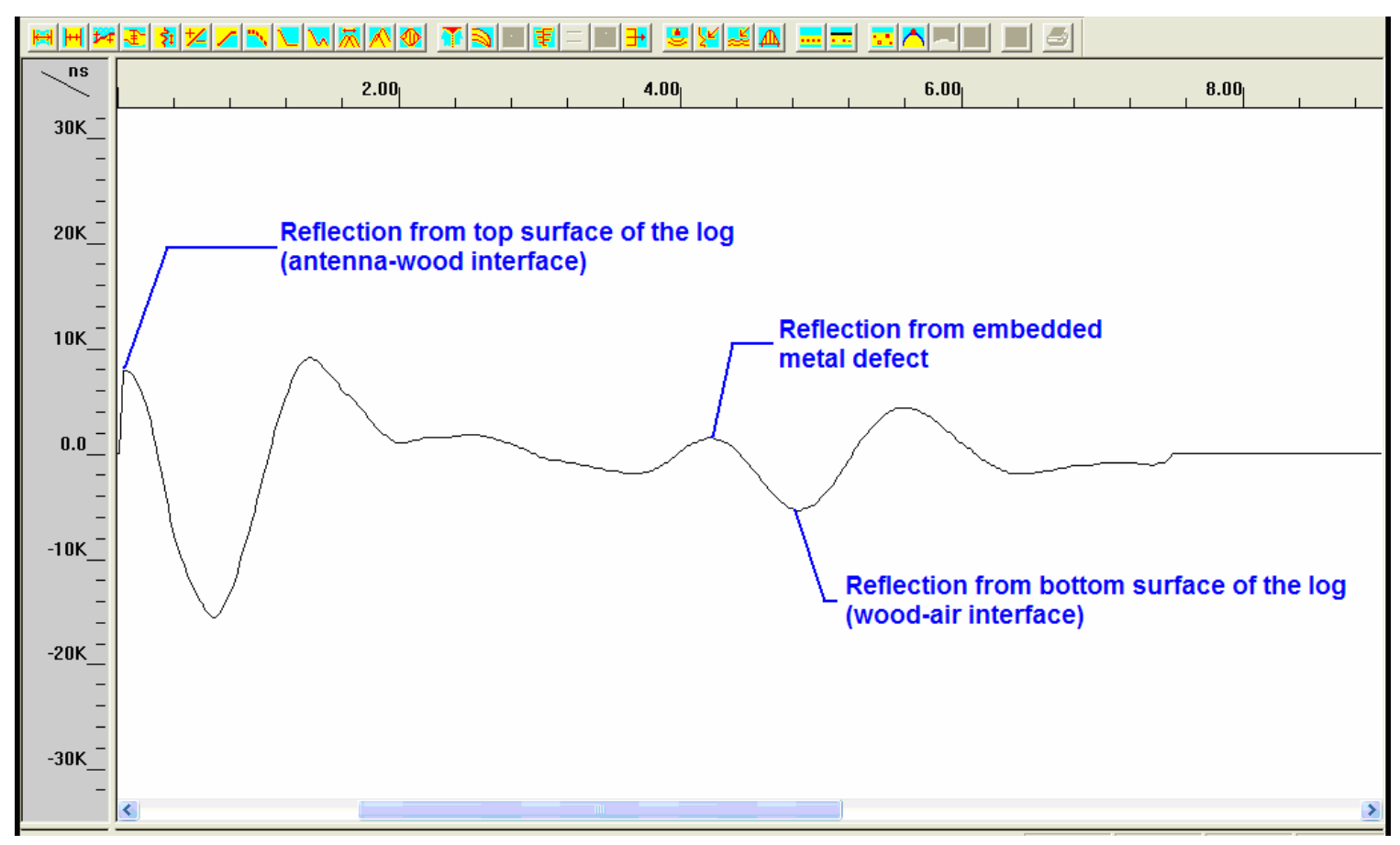

(a) 


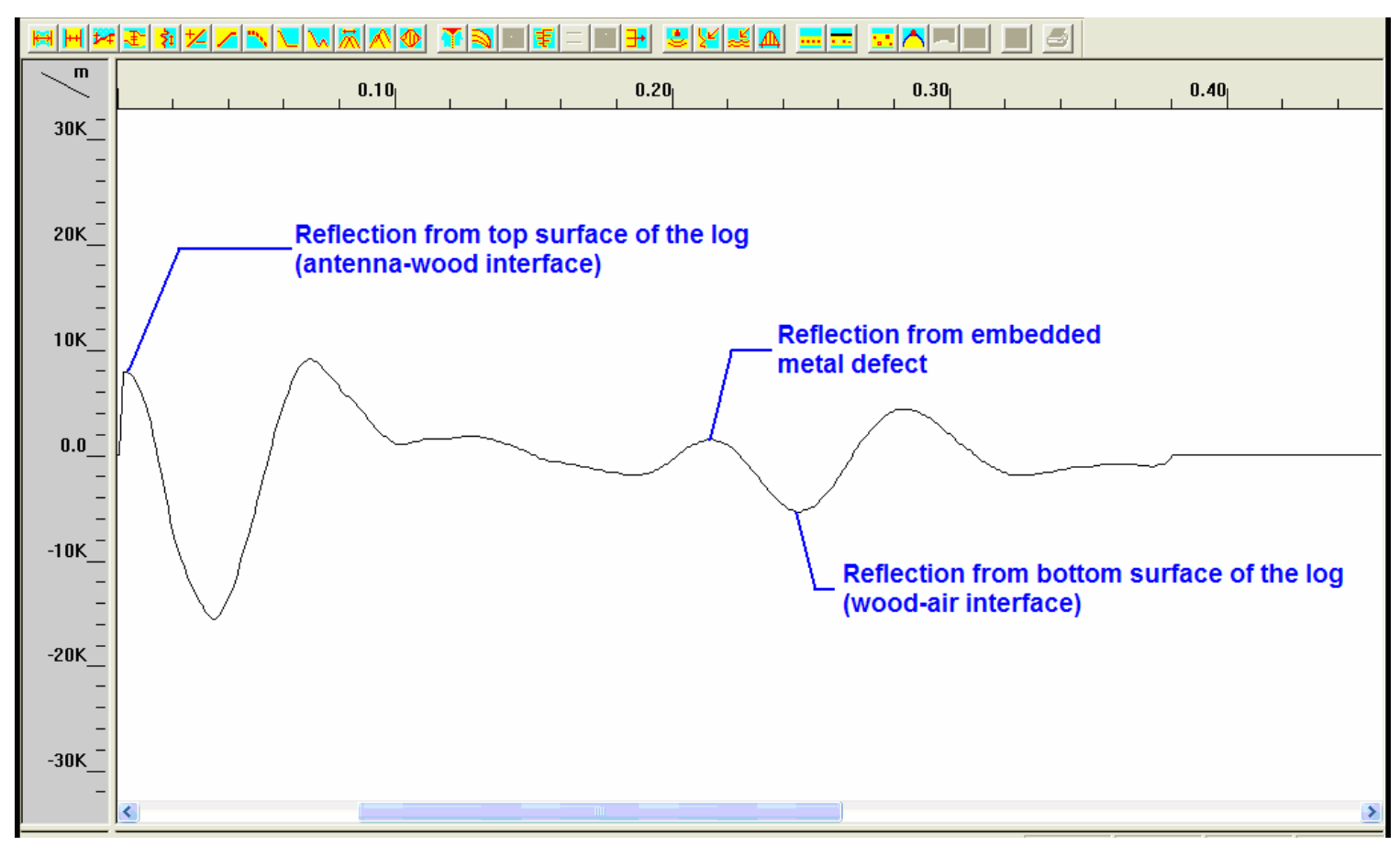

(b)

Figure 4-11 A typical radar signal (viewed in oscilloscope mode) after applying zero correction with the horizontal scale showing (a) two-way travel time (ns) (b) depth (m)

After applying the zero correction the data must be filtered. Filtering removes direct coupling surface reflections, flat-lying ringing system noise and snow like peaks. The flat-lying ringing system noise is characterized by flat-lying horizontal bands, usually with lower frequency than that of the real reflections in the data set. This type of noise is usually most prevalent when the range is set near the maximum limits for antenna. Another type of noise is the high frequency noise which result in "snow-like" noise in the data, which is most prevalent when the range is set near the maximum limits for antenna or when large amount of gain is used.

The noise in the acquired data can obscure real reflection near the surface or at greater depth. These noises can be removed by using background removal (horizontal high pass filtering), vertical high frequency filter, and vertical low frequency filter. These features are all available under the same tool called Finite Impulse Response (FIR) filter. Figure 4-12 shows the values applied for background removal, high frequency filter, and low frequency filter. The figure shows a choice between Boxcar and Triangle filters (both 
are under FIR filter). The Boxcar filter is a rectangular window function while the Triangle filter emphasizes the center of the filter more heavily than the ends of the filter (GSSI 2002b). The Boxcar filter was chosen for this research since it provided a uniform window function. The GPR scan looks considerably clearer after the FIR filter was applied, as shown in Figure 4-13.

Background removal is done by applying Horizontal High Pass (Background Removal) Filter. This process removes the flat-lying or horizontal bands of ringing noise which usually has frequency lower than that of real reflection in the data set. High pass horizontal filters provide the best way of removing these kinds of ringing noise. This filtering also removes the surface reflection (direct coupling) pulse. The background removal number is set to one less than the number of sample points in each waveform $(512-1=511$ points $)$.

The Horizontal filter also provides a Stacking option, which is a Horizontal Low Pass filter designed to remove high frequency "snow like" noise. The system manufacturer suggests using a odd number value, typically 5 (GSSI 2002b). Also, the software allows you to do either the Background Removal or the Stacking process (and not both) in one run. This can only be done in successive filtering runs. This is why the stacking value is set to zero, implying no stacking, in Figure 4-12.

The Vertical High Pass Filter with a cut-off frequency corresponding to about 1/3 the center frequency of the antenna $(1 / 3 \times 900 \mathrm{MHz}=300 \mathrm{MHz})$ is used if the desired horizontal features are of higher frequency content (i.e., low frequency noise is removed).

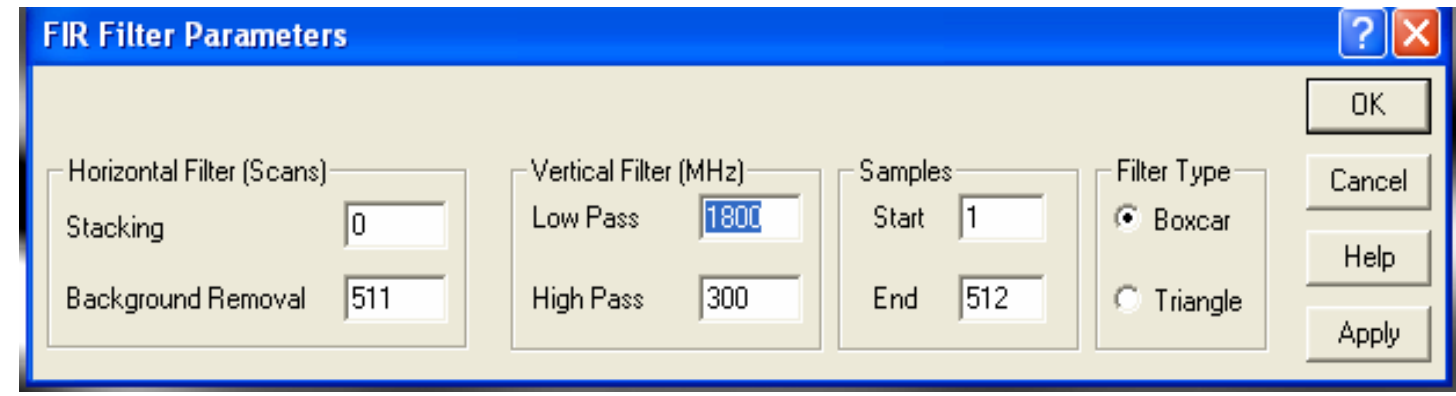

Figure 4-12 FIR filter parameters for Background removal and vertical Low and High pass filtering 
The Vertical Low Pass filter is set to a cut-off frequency that is twice the center frequency of the antenna $(2 \times 900 \mathrm{MHz}=1800 \mathrm{MHz})$. Low pass filter reduces the high frequency "snow like" noise.

Figure 4-13 shows the processed data after filtering, and Figure 4-14 shows a typical processed radar signal in the oscilloscope mode. After processing, the initial header file showing the data collection settings and parameters (Figure 4-3) is now modified into a new header file, which displays the data collection parameters as well as the filter values that were applied. This new header file is shown in Figure 4-15, and it can be seen that the input filter parameters are adjusted slightly by the software.

From Figure 4-14 it can be recognized, that the initial high amplitude peak due to surface coupling was removed and some other smaller peaks became more predominant. It is important to note that filtering also has the disadvantages of adding unwanted signals and also removing desirable features from data. Therefore, one has to carefully study both the unfiltered and filtered data during the analysis process, and adjust the filtering parameters as necessary.

The above basic processing steps (range gain, zero correction, background removal and noise filtering) were applied to all the data sets in this research. RADAN software provides other specialized tools where more complex processing steps could be applied for finer refinement of data. One such tool is Deconvolution of data, which can be done before the FIR filtering process. Deconvolution usually reduces multiple reflections or ringing between interfaces and helps in resolving closely spaced layers. Deconvolution was attempted for GPR data for some of the logs but instead of helping it actually added unwanted features. Therefore, this tool was not used for subsequent research. 


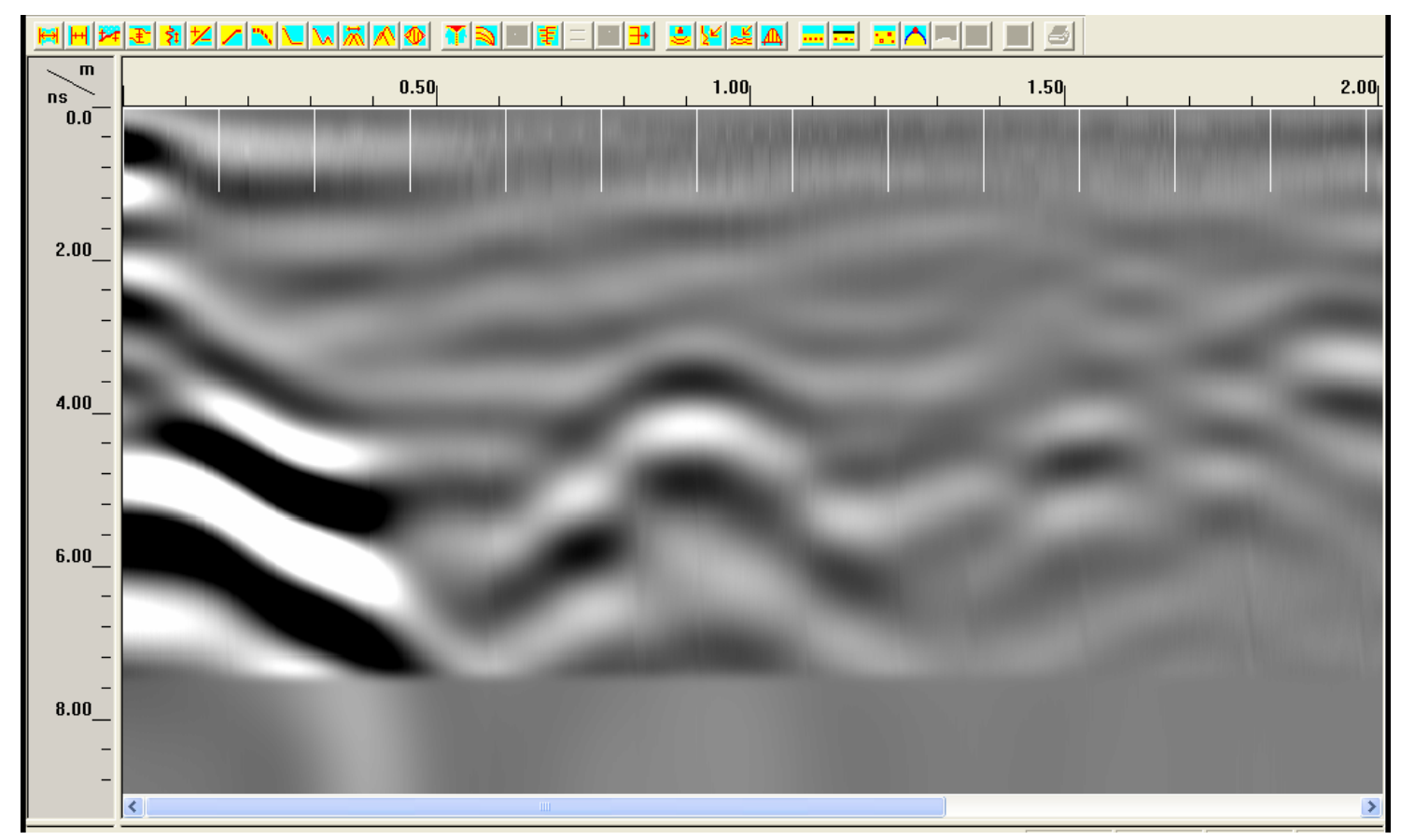

(a)

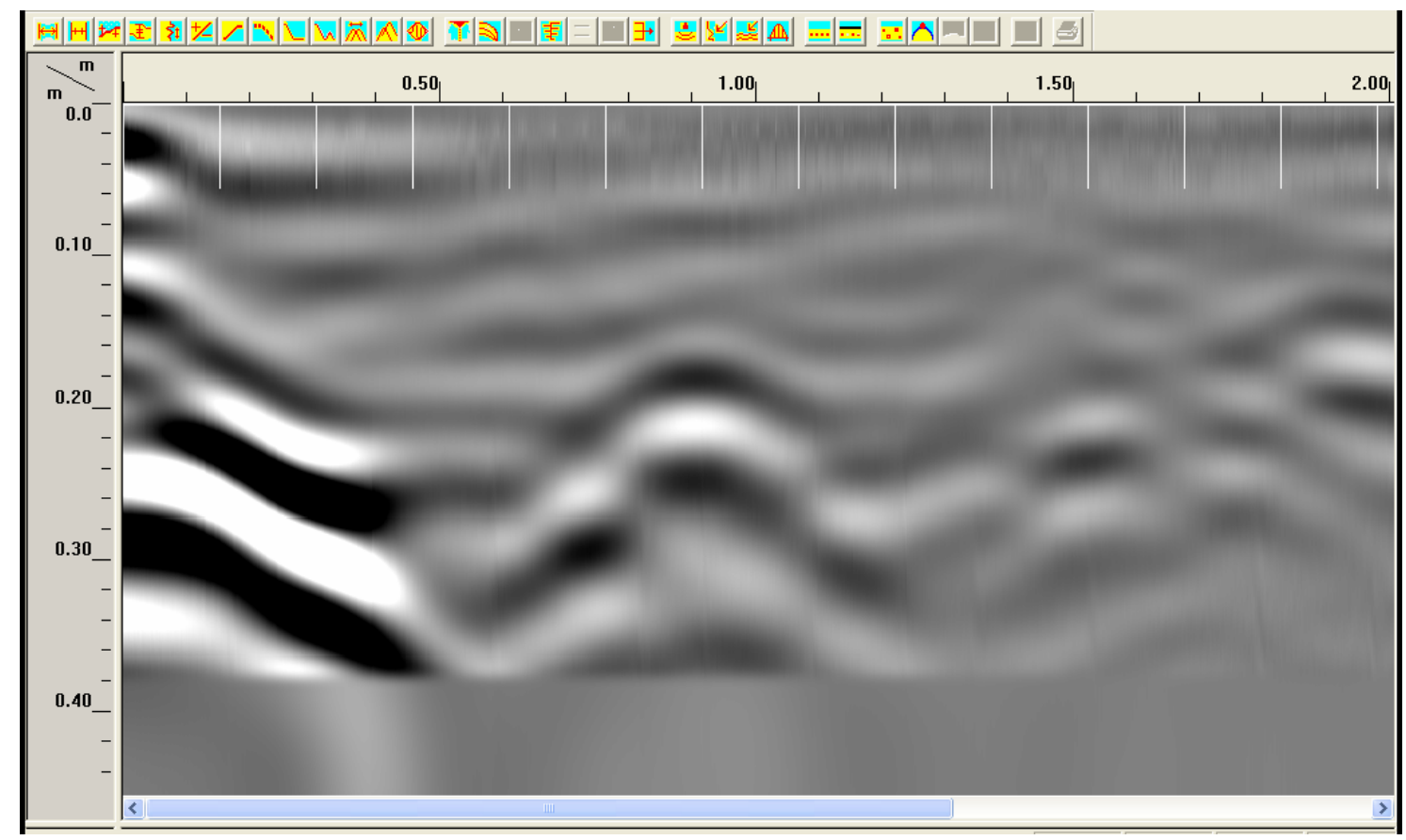

(b)

Figure 4-13 GPR data after applying background removal and high pass and low pass filters (a) time (ns) plot (b) depth (m) plot 


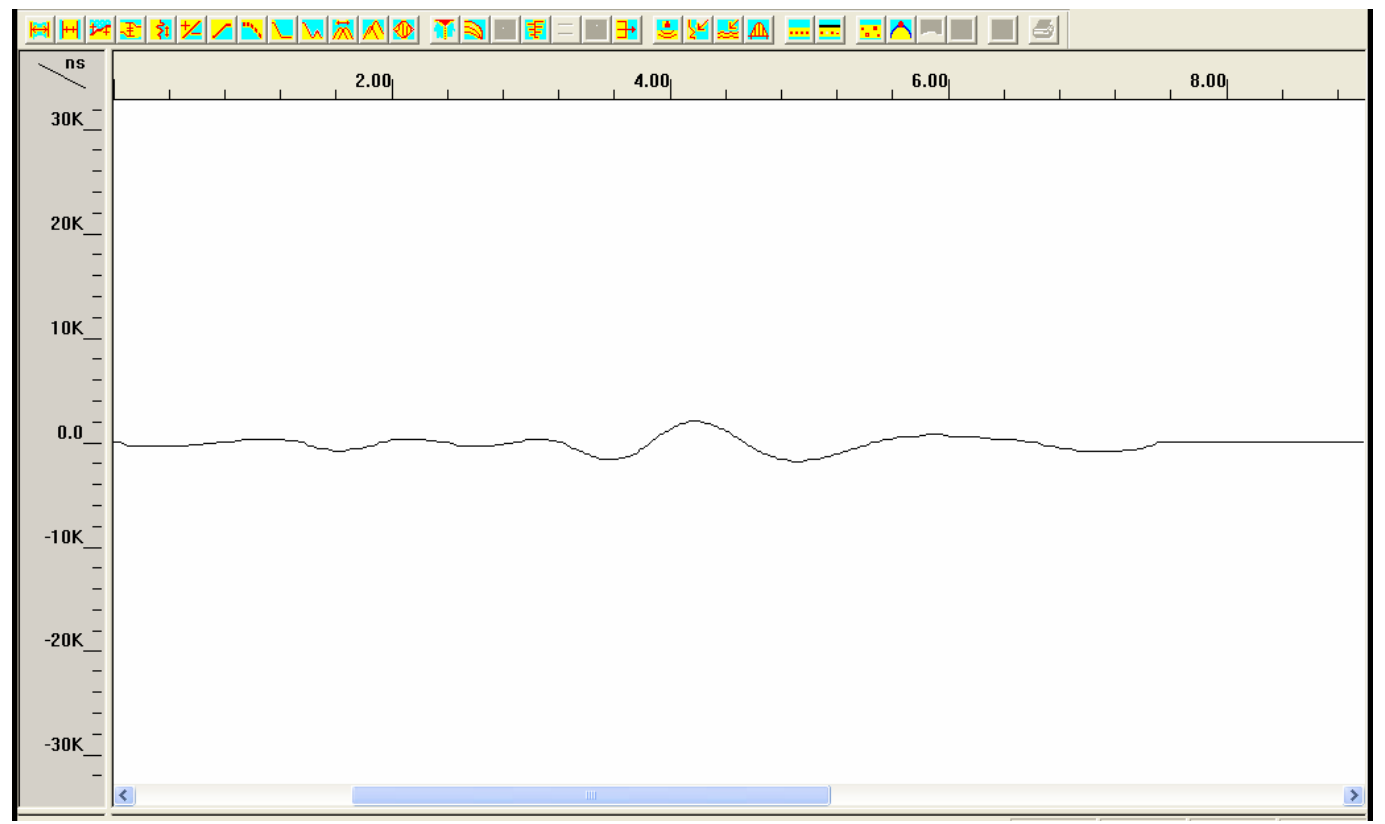

Figure 4-14 A typical radar signal (observed in oscilloscope mode) after applying background removal and FIR filtering

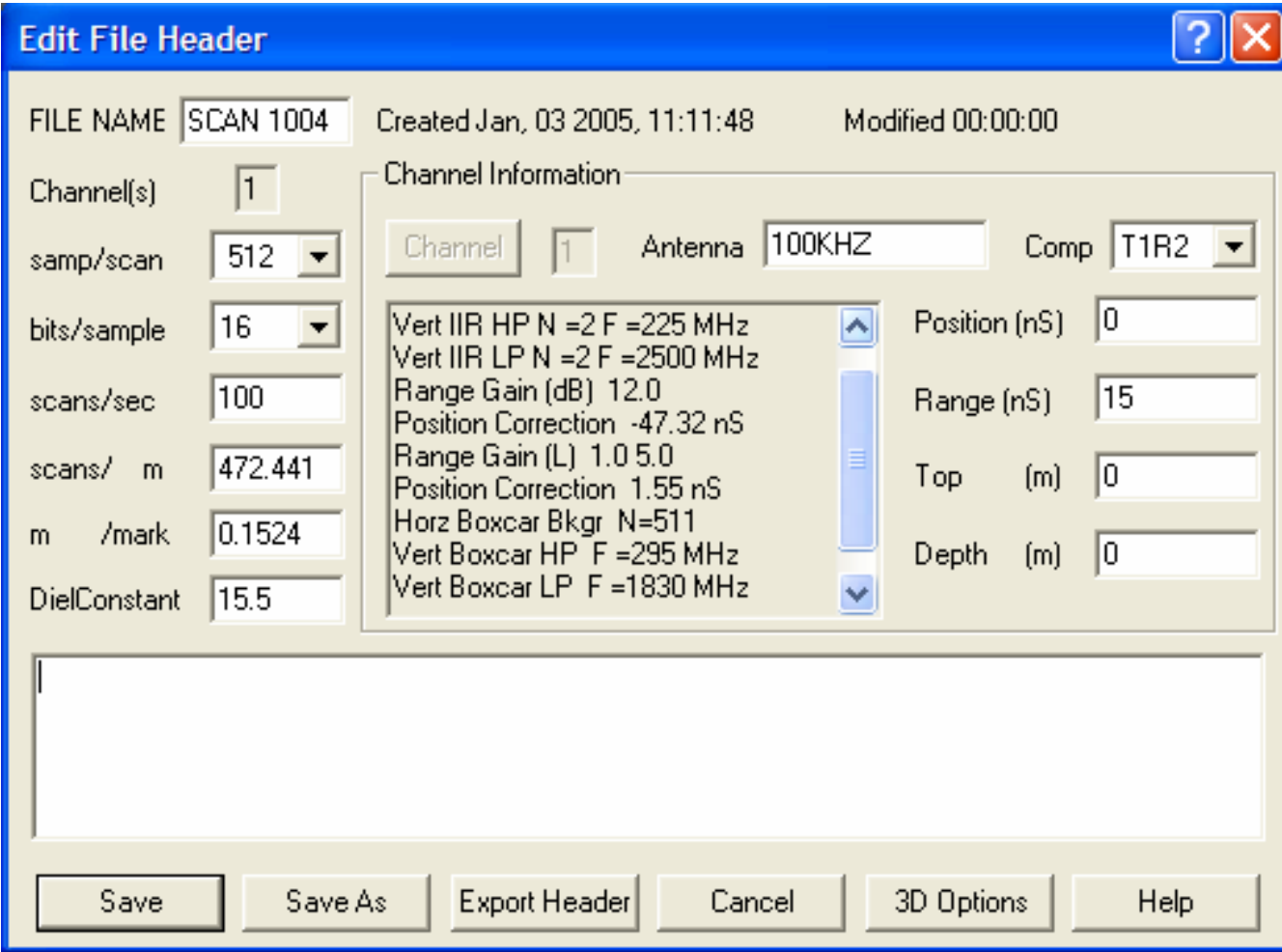

Figure 4-15 Data collection parameters 


\subsection{MEASUREMENT OF DIELECTRIC CONSTANT}

To locate the depth of the internal characteristics (defects and embedded metals), it was necessary to input the correct dielectric constant of the log. The actual dielectric constant value varies with moisture content along the depth of the log with lower values near the log's surface and higher values towards the center. Using the measurement for the diameter of the log and the two-way signal travel time through the log, it is possible to compute the average dielectric constant value for the log using Equation 4.1.

$$
\sqrt{\varepsilon^{\prime}}=\frac{V_{0}}{d /(t / 2)}=\frac{V_{0} t}{2 d}
$$

where,

$$
\begin{aligned}
& \varepsilon^{\prime}=\text { dielectric constant } \\
& V_{0}=\text { velocity of radar signal in vacuum }\left(=3 \times 10^{8} \mathrm{~m} / \mathrm{s}\right) \\
& d=\text { diameter of } \log (\mathrm{m}) \\
& t=\text { two-way travel time from the } \log \text { surface to the bottom of the } \log (\mathrm{ns})
\end{aligned}
$$

Figure 4-16 shows a radar signal viewed in the RADAN oscilloscope mode. This signal was acquired from a log without placing aluminum foil at the bottom. Therefore, the bottom echo (from wood-air interface) has a reverse polarity compared to the top echo (antenna-wood polarity). The distance between the first positive peak and the bottom negative peak gives the two-way travel time $(t)$ through the log. Once the diameter of the $\log (d)$ is measured at the corresponding location, Equation 4.1 can be used to compute the dielectric constant. This value represents the average value of the dielectric constant over the measured cross-section of the log. Using this value, the depth of the subsurface defects could be located, but with a small error (see chapter 5 for details) which is caused because the true dielectric constant value varies with depth within the log. 


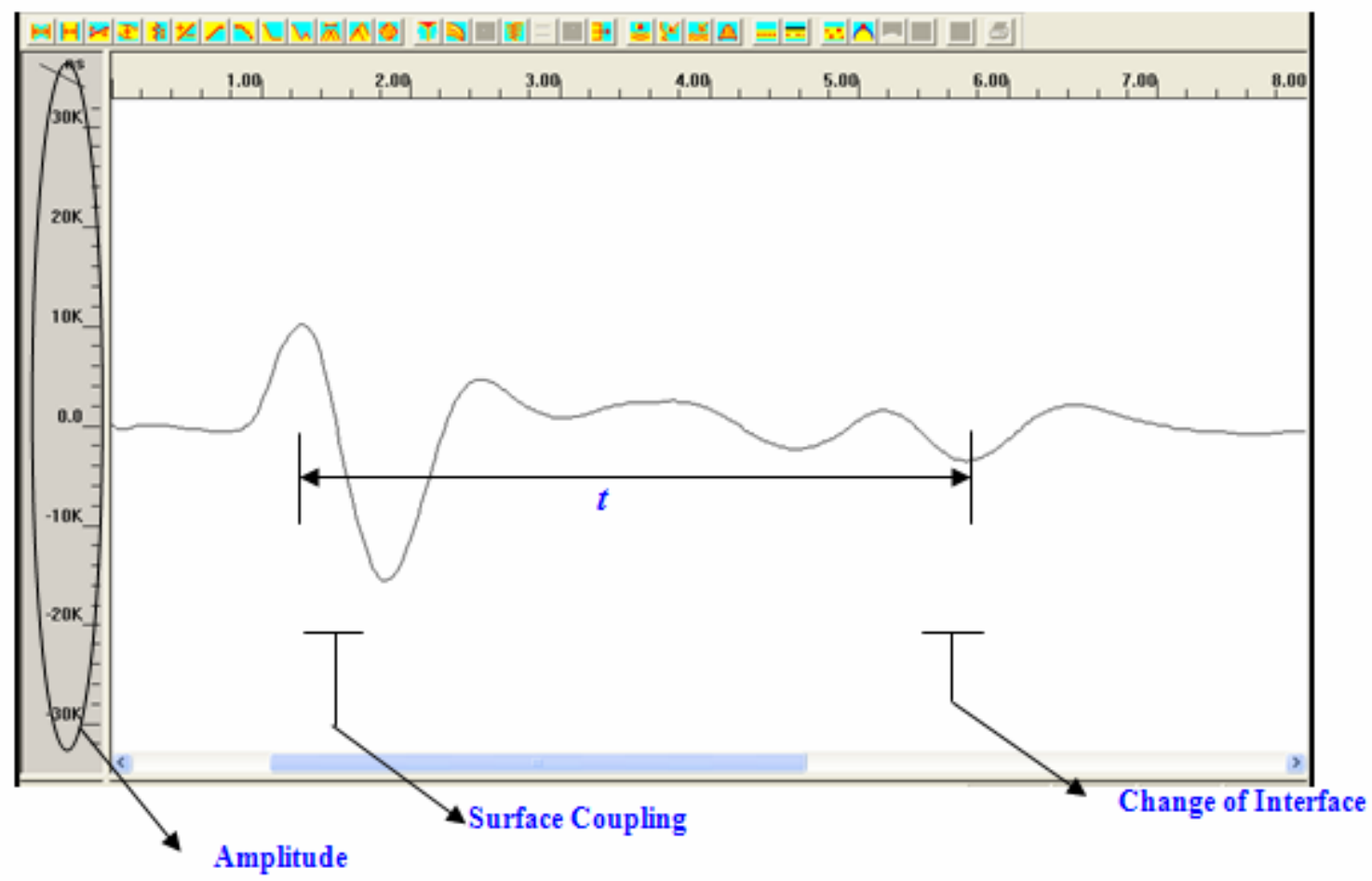

Figure 4-16 GPR signal in RADAN oscilloscope mode used for measuring the dielectric constant 


\section{Chapter 5 \\ EXPERIMENTAL RESULTS}

This Chapter describes the experimental results obtained from GPR scanning of the all the logs using the set up described in Chapter 3. For each log, detailed analysis of the results using the procedure described in Chapter 4 is also presented. A total of seven logs were tested. Additional tests were also conducted to determine interference between antennas if multiple antennas were to be used.

Each log was tested after it was debarked. After each log was scanned using GPR, all the processed scans from the log was printed. Portions of the GPR scans which showed different characteristics (e.g., high amplitude signal clutters) were marked on the printouts as possible defects. Each log was then sawed at the corresponding locations, and the visual observation of defects was correlated with the markings on the GPR scans. This helped establish the ability of GPR to detect different types of defects (embedded metals, knots, decays or rots).

From the logs tested, it was observed that the diameter varied from one end of the $\log$ to the other end. The end with the larger diameter is called "butt end." Also, the length and diameter of all the logs were different.

The moisture content for each $\log$ was measured at 10 points along its thickness (near surface to center of the log) using a digital moisture meter. This measurement was made after sawing the log, since the internal moisture content values are more representative of the entire log and are free from errors due to evaporation of moisture at the ends. The measured moisture content for various logs varied from 10\% to $99 \%$ from surface to center of the log.

The travel speed of the radar signal through each log was calculated from the GPR scan and the dielectric constant was estimated using the procedure described in Section 4.3. The correct dielectric constant was applied to each scan before processing the data. 


\subsection{LOG 1}

Table 5-1 Characteristics of $\log 1$

\begin{tabular}{|c|c|c|c|c|c|c|c|c|}
\hline Log & Species & $\begin{array}{c}\text { Length } \\
\text { no. }\end{array}$ & \multicolumn{2}{|c|}{ Diameter (m) } & \multicolumn{2}{|c|}{ Moisture Content (\%) } & Dielectric \\
& Butt & $\begin{array}{c}\text { Small } \\
\text { end }\end{array}$ & Surface & Max. & Average & Constant \\
\hline 1 & $\begin{array}{c}\text { Yellow } \\
\text { Poplar }\end{array}$ & 2.45 & 0.35 & 0.33 & 16 & 36 & 24 & 12 \\
\hline
\end{tabular}

The characteristics of $\log 1$ are given in Table 5-1. The log scanning set up is shown in Figure 5-1. Two antennas were used simultaneously for collecting data, with each antenna transmitting and receiving its own signal. The log had fairly uniform diameter and smooth surface. The bark from the log was removed before scanning the $\log$. The moisture content values in Table 5-1 report the maximum, minimum, and average of measurements taken at 10 points across the log's thickness. The data collection and processing parameters for $\log 1$ are shown in Figure 5-2. These parameters are explained in detail in Sections 4.1 and 4.2.

Figure 5-3 shows a raw GPR scan with the horizontal axis showing the distance traveled by the GPR antenna along the surface (length) of the log. The vertical axis shows either the two-way travel time in nanoseconds (Figure 5-3a) or depth in meters (Figure 5$3 b)$ in the log. The top scan in each of the figures is from the top antenna and the bottom scan is from the side antenna. These scans represent a collection of signals acquired by the antennas as they were moved along the logs surface. The actual radar signals were converted by the software to grey scale scans (Figure 5-3) where the intensity in the scan is a measure of signal amplitude with white bands for positive signal peaks and black bands for negative signal peaks. The intensity of the band is a measure of the signal amplitude. The top set of white, black and white band indicates the transmit signal (antenna to log coupling). Echoes from internal interfaces show up as bands arriving later in time, which are also of lower intensity due to signal attenuation and reflection/transmission losses within the log. 
It can be seen from Figure 5-3 that the horizontal bands from the interior of the log are curved and undulated indicating changes in travel time or depth of the bands from the surface. Changes in the bands, which correspond to changes in the internal characteristics (subsurface defects) of the log, could be identified at distances of $0.9 \mathrm{~m}$ and $1.65 \mathrm{~m}$ along the surface of the $\log$ as shown in Figure 5-3 (a). The bottom of the $\log$ ( $\log$ to air interface) was visible at 8-9 ns, and the corresponding band is undulated (not perfectly horizontal) due to variation in moisture content, dielectric constant, wave velocity, and internal characteristics (defects) within the log. The bottom reflection tends to bulge out due to the presence of defects in any given cross-section of the log (e.g., at $0.9 \mathrm{~m}$ and $1.65 \mathrm{~m}$ along the length of the $\log$ ).

The average dielectric constant for the log was calculated following the procedure described in Section 4.3. This value was obtained as 12 and was used to convert the vertical time axis in Figure 5-3(a) to vertical depth axis in Figure 5-3(b). The detection of internal features from the raw data is usually difficult. Therefore, the raw data was processed using the procedures described in Section 4.2 in conjunction with the parameters shown in Figure 5-2(b).

After the data was processed, all the internal defects were predicted based on location of the signal clutter (changes in signal bands) in the processed data shown in Figure 5-4. It is important to note that the time and depth axis in this figure have all shifted upwards in comparison to Figure 5-3 because of the zero correction (see Section 4.2.2 for details). The depth axis in Figure 5-4 now corresponds to actual depth from the logs surface. Also, the signal clutters are more pronounced compared to the raw data in the Figure 5-3. The bottom of the $\log$ is visible in Figure 5-4(a), top antenna scan, at around 7 to $8 \mathrm{~ns}$, with the corresponding depth in Figure 5-4(b) being about $0.3 \mathrm{~m}$. There is an echo from the center of the $\log (3.5 \mathrm{~ns}$ or $0.15 \mathrm{~m}$ in the top antenna scan) starting from the butt end extending all the way to the small end. This was due to rot-initiation and high moisture content as indicated in Figure 5-4 (a). Other subsurface defects are also shown in the GPR scans in Figure 5-4 (a), which shows both antennas. It should be noted in Figure 5-4 (a) that while all defects showed up well in the top antenna scan, only some of the defects show up in the bottom antenna scan. The defects which did not show up are either too close to the surface (one of the knots in Defect 2) or too far (Defect 4 - near the 
bottom interface) or possibly outside the angle of the antenna's EM beam (Defect 3) as can be seen from Figure 5-6. This indicates the need for taking multiple scans around the $\log$.

The top scan in Figure 5-4 (b), which shows the depth plot for the top antenna, has been reproduced in Figure 5-5. The log was sawed at positions where processed data indicated changes in signal characteristics, which were attributed to presence of internal defects. The cut circular cross sections are shown in Figure 5-6 where the blue circles in the center indicates rot initiation and the black circles indicates knots. There was a good correlation between the changes observed in the signal characteristics and the actual location of defects observed after the sawing of log. The predicted rot initiation was at about $0.16 \mathrm{~m}$ as seen from Figure 5-5 and the actual depth measured after sawing was 0.15 to $0.16 \mathrm{~m}$. The knot (Defect 1) in Figure 5-6 (a) shows up in the GPR scan (Figure $5-5)$ at $0.26 \mathrm{~m}$ depth whereas the actual defect is at about $0.25 \mathrm{~m}$. It should be noted that GPR detects the top of the defect (wood-defect interface) within the angle of the signal beam. For knots on the side (Defect 2 in Figure 5-6(b)), the distance to the knot is at an angle, and the echoes from the two knots came successively. Reflection from the first knot (Defect 2) arrived at $0.18 \mathrm{~m}$ and merged into the reflection from rot initiation. Reflection from the second knot has merged into the bottom reflection, which makes it difficult to resolve the second knot from the GPR scan. The measured distances of the two knots in Figure 5-6(b) from the top antenna were $0.18 \mathrm{~m}$ and $0.29 \mathrm{~m}$. Also, it can be seen in Figure 5-5 that the bottom reflection has shifted down at locations where knots are encountered, which is because these defect regions have higher moisture content and higher dielectric constant. For Defect 3, the predicted depth from the GPR scan (Figure 55) was $0.22 \mathrm{~m}$ where as the actual depth (Figure 5-6(c)) was $0.23 \mathrm{~m}$. For Defect 4 , the predicted depth from the GPR scan (Figure 5-5) was $0.2 \mathrm{~m}$ where as the actual depth (Figure 5-6(c)) was $0.23 \mathrm{~m}$. The above results show that the resolution of depth prediction is 0.01 to $0.04 \mathrm{~m}$. As mentioned in Section 2.6, the resolution value is equal to approximately half the wavelength, which comes to $0.048 \mathrm{~m}$ for $900 \mathrm{MHz}$ radar signal in a $\log$ with dielectric constant of 12 . Also, the defects in the sawed sections correlated very well with the GPR scans, which indicate that the prediction of defective sections along the length of the $\log$ is very accurate. 
Figure 5-7 (a) and (b) show the wiggle plots (also known as "wiggle traces") of raw and the processed data, respectively. In the wiggle format the GPR data, consisting of successive radar signals, are displayed as actual waveforms instead of grey scale intensity scans. The vertical axis corresponds to depth (or time) and the horizontal scale represents the distance traveled by the antenna along the surface of the log. Each wiggle trace shown in the wiggle plot is an average of 16 individual radar waveforms (GSSI $2002 b$ ). From the wiggle plot the variation of signal amplitude at different points along the length and depth of the data can be observed, and these variations indicate presence of internal characteristics (defects). While wiggle plot represents the same data as grey scale scans, it may sometimes be useful for more accurate estimation of defect depth based on the location of high amplitude peaks and corresponding phase changes. However, the processed wiggle plot (Figure 5-7(b)) did not show the defect locations as clearly as the GPR scans in Figure 5-4 (b) and Figure 5-5, so the wiggle plots were not used any further. This conclusion was found to be true for other logs as well as discussed later.

From the processed GPR scans, the individual waveforms for defective sections (with knots) were extracted and compared with a waveform from a knot-free section. The waveform comparisons are shown in Figures 5-8 (a) for raw data and Figures 5-8(b) and 5-8(c) for processed data. Figures 5-8(a) and (b) show the waveform amplitudes on the vertical axis and the two-way travel time through the depth of the log on the horizontal axis. Figure 5-8(c) shows the waveform amplitudes on the vertical axis and the travel distance through the depth of the log on the horizontal axis. The raw waveforms in Figure 5-8(a) show very little difference between the signals. On the other hand, the processed data in Figures 5-8(b) and 5-8(c) shows several key features. For the knot-free waveform, the first positive peak at $0 \mathrm{~ns}(0 \mathrm{~m})$ and the second positive peak at $1.46 \mathrm{~ns}(0.7 \mathrm{~m})$ represent the surface reflection. The feature beginning at around $3.5 \mathrm{~ns}(0.16 \mathrm{~m})$ corresponds to the rot initiation. The rot initiation reflections also show up in the Defect 1 and 2 waveforms. In addition, Defects 1 and 2 waveforms show additional prominent peaks indicating the presence of knots. Defect 1 is a knot at $0.26 \mathrm{~m}$ depth where as the measured depth was $0.25 \mathrm{~m}$. Defect 2 knots are marked at $0.18 \mathrm{~m}$ and $0.29 \mathrm{~m}$ in Figure 5$8(\mathrm{c})$, which are the exact depths obtained from sawing. This shows that use of actual signals (Figures 5-8 (b) and (c)) instead of GPR scans (Figures 5-4 and 5-5) is more 
accurate in depth prediction. However, extracting and analyzing individual signals is a time consuming process.

In Figures 5-8(b) and 5-8(c), the bottom reflection appears around 7 to $8 \mathrm{~ns}(\sim 0.3$ m) for all three waveforms. It should be noted that the bottom peak has undergone phase reversal since it is a wood-air interface.

Overall there was a very good correlation between the location of defects predicated from GPR data and their actual position in the log observed after sawing.

The GPR scans from the two antennas indicated that some defects which were detected from the first antenna position (top) could not be detected using the second antenna position (side). This indicates the need to use multiple scanning positions around the log and then combine the data into a 3-D image.

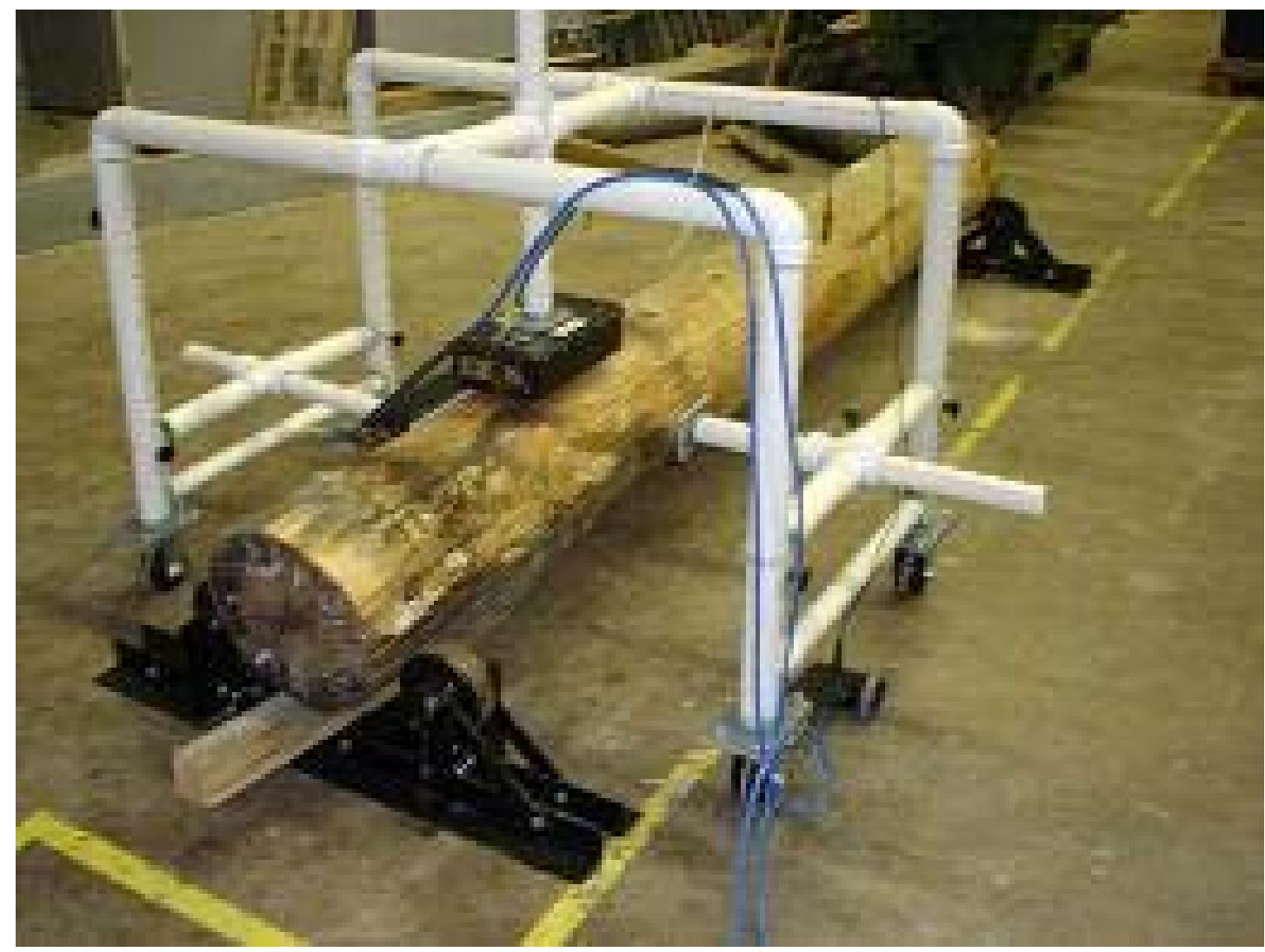

Figure 5-1 Antenna configuration for Log 1 


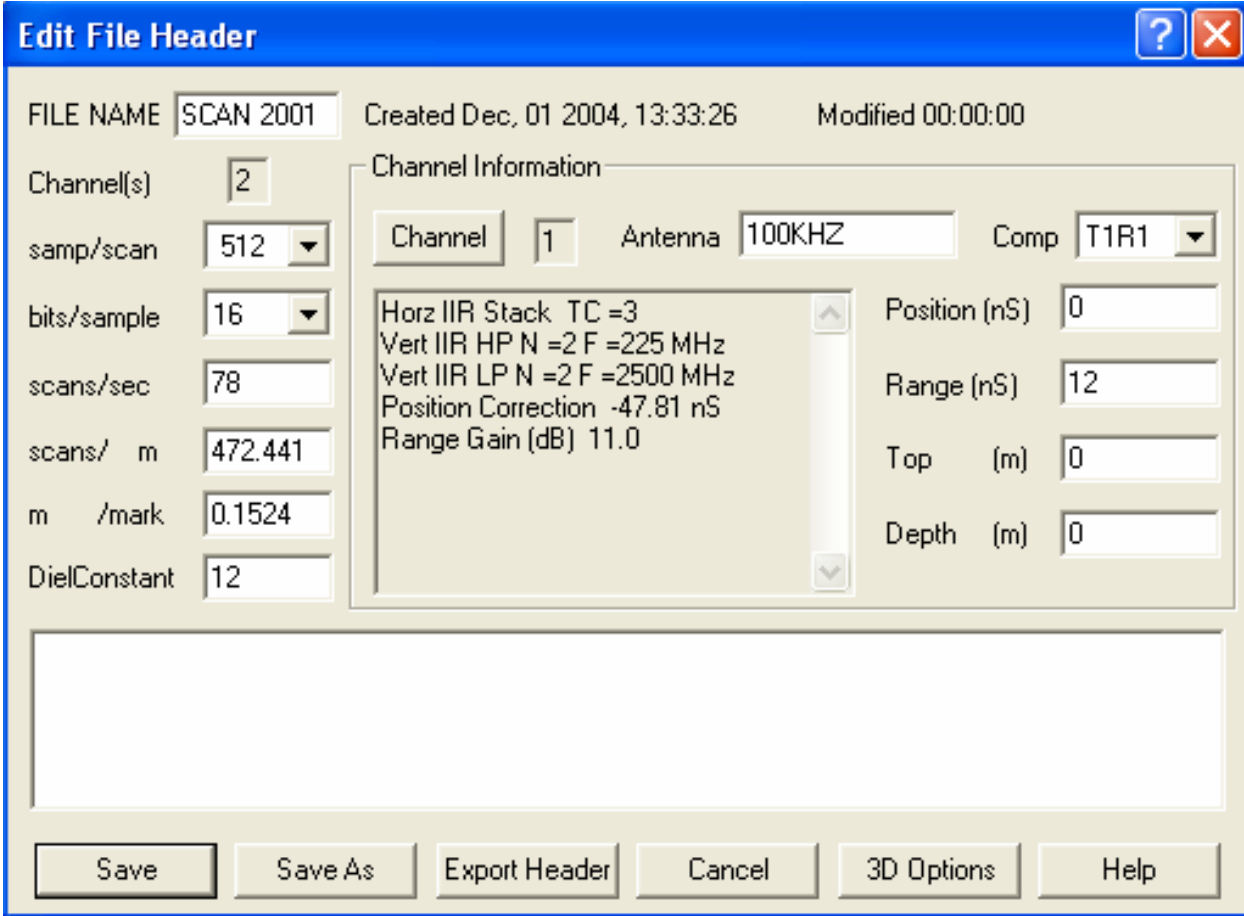

(a)

\section{Edit File Header}

FILE NAME SCAN 2001 Created Dec, 01 2004, 13:33:26 Modified 00:00:00

Channel[s] $\quad \longdiv { 2 }$

samp/scan 512 .

Channel Information

\begin{tabular}{ll}
\cline { 2 - 2 } bits/sample & $16 \quad$ \\
scans/sec & 78 \\
scans/ m & 472.441 \\
$\mathrm{~m} /$ mark & 0.1524 \\
DielConstant & 12
\end{tabular}

Channel $\sqrt{1} \quad$ Antenna $\sqrt{100 \mathrm{KHZ}}$ Comp $\longdiv { T 1 R 2 \quad - }$

Vert IIR LP N $=2 \mathrm{~F}=2500 \mathrm{MH}$

^ Position [nS] 0

Position Correction $-47.81 \mathrm{nS}$

Range Gain [dB] 11.0

Range Gain (L) 0.05 .0

Position Correction $0.96 \mathrm{nS}$

Horz Boxcar Bkgr $N=511$

Vert Boxcar HP $\mathrm{F}=300 \mathrm{MHz}$

Vert Boxcar LP F $=1830 \mathrm{MHz}$

Range (nS) 12

Top [m] 0

Depth (m) 0

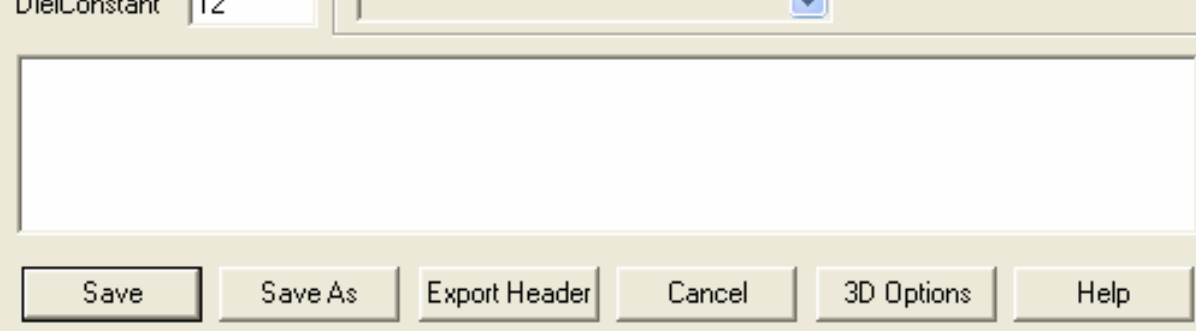

(b)

Figure 5-2 (a) Data collection parameters (b) data processing parameters 


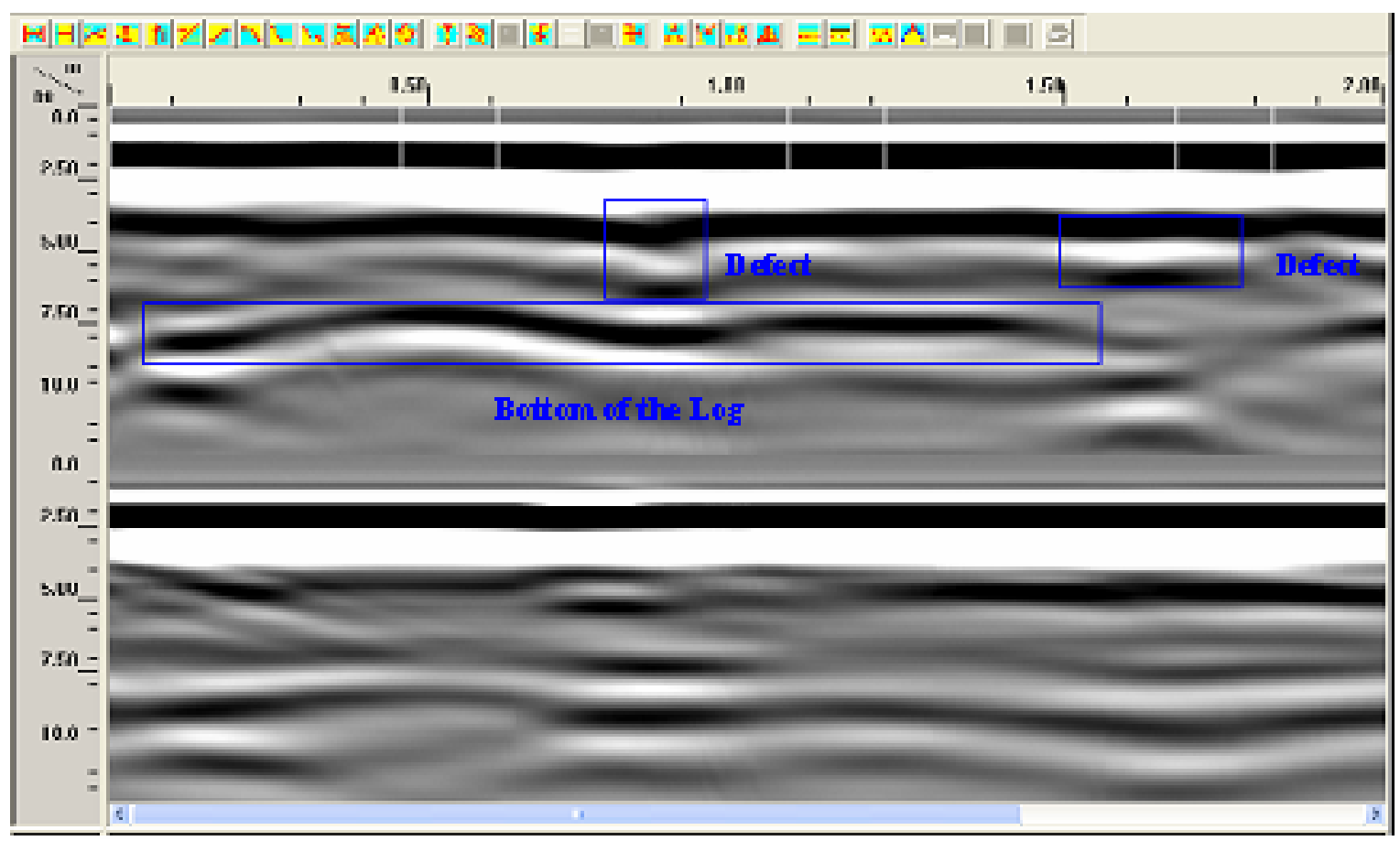

(a)

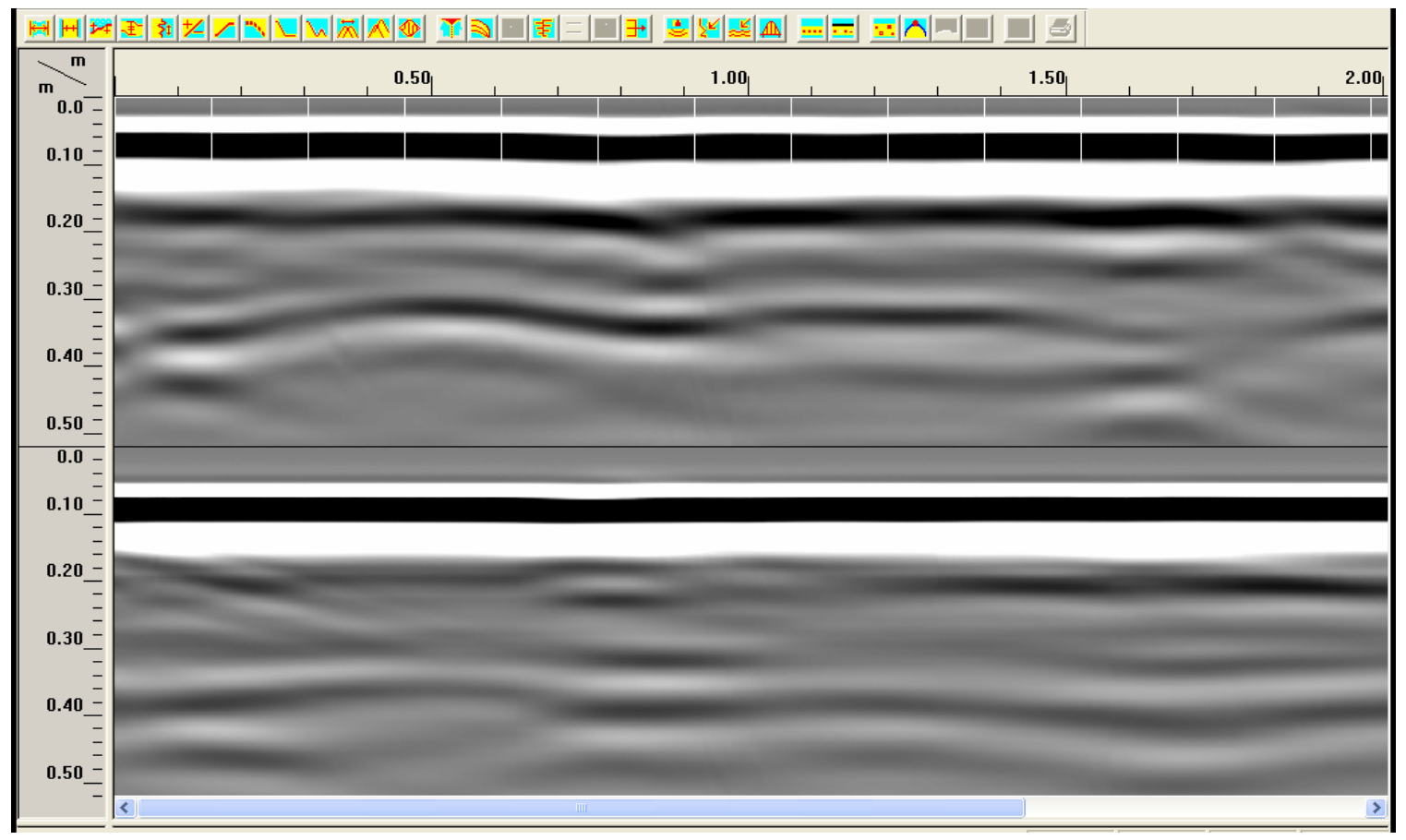

(b)

Figure 5-3 Raw GPR data of $\log$ 1, horizontal scale indicating the travel distance along the length of the $\log (\mathrm{m})$ and the vertical scale indicating (a) time (ns) (b) depth (m) 


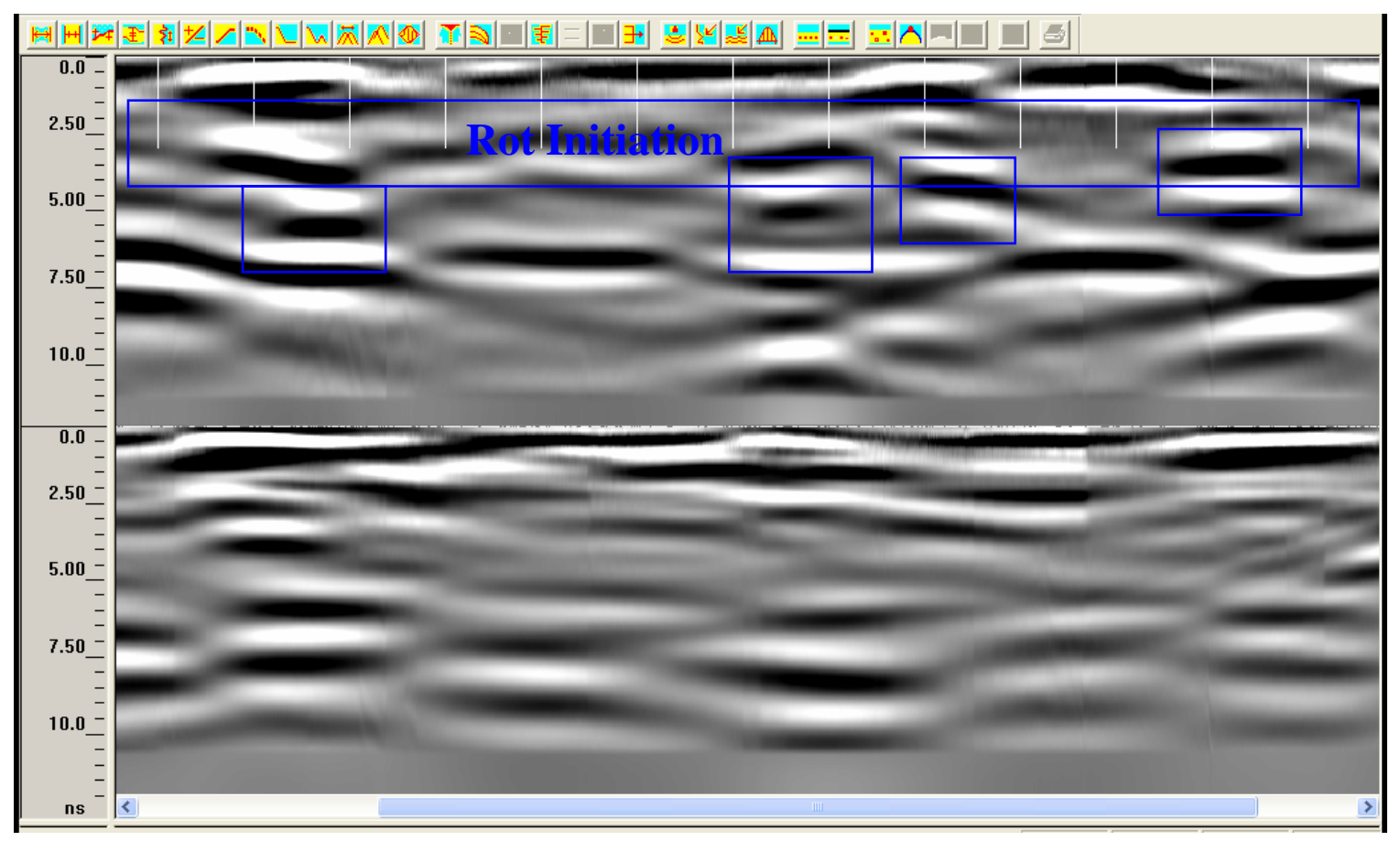

(a)

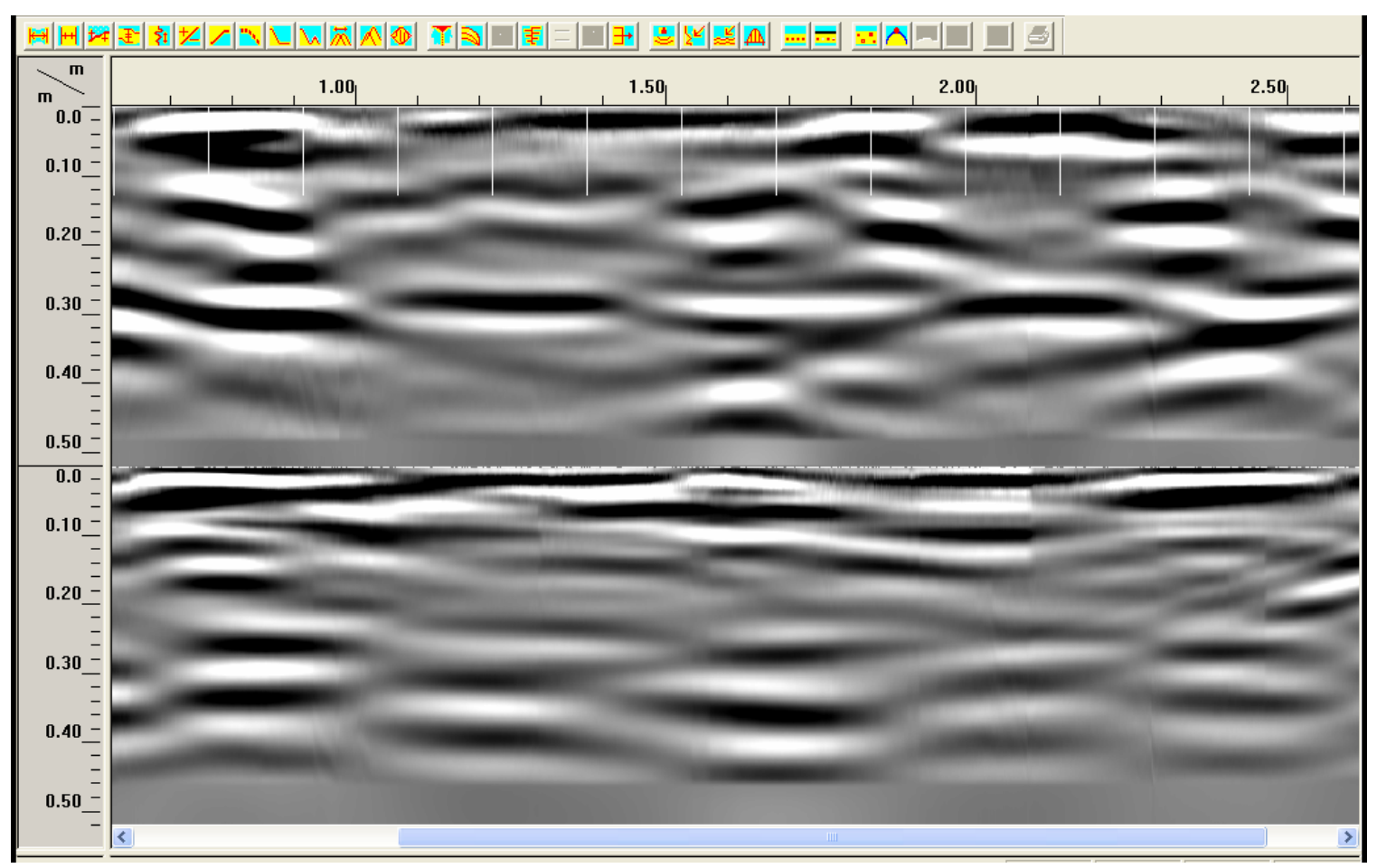

(b)

Figure 5-4 Processed GPR data of Log 1, horizontal scale indicating the travel distance along the length of the $\log (\mathrm{m})$ and the vertical scale indicating (a) time (ns) (b) depth (m) 


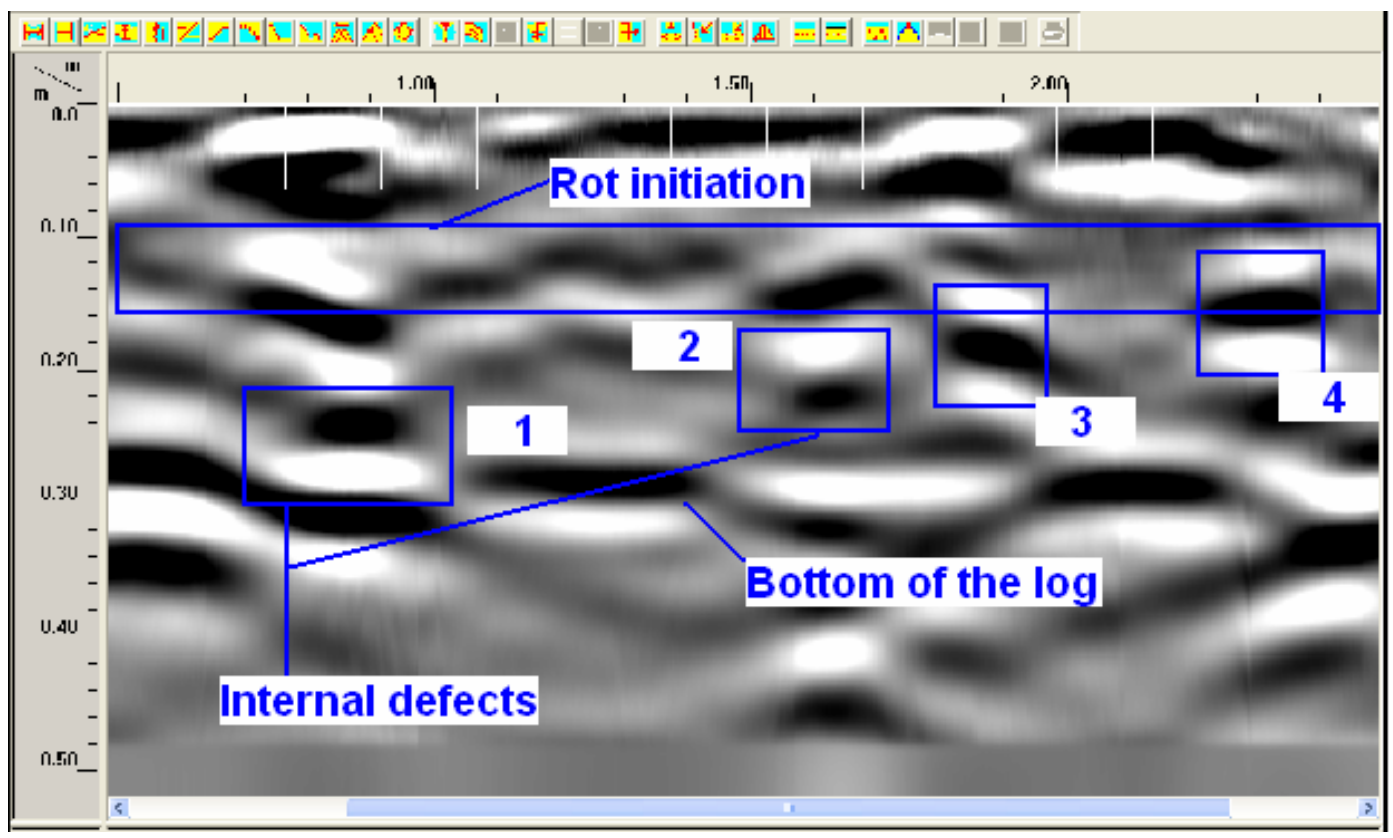

Figure 5-5 Processed GPR data of $\log 1$ indicating the internal features of $\log 1$

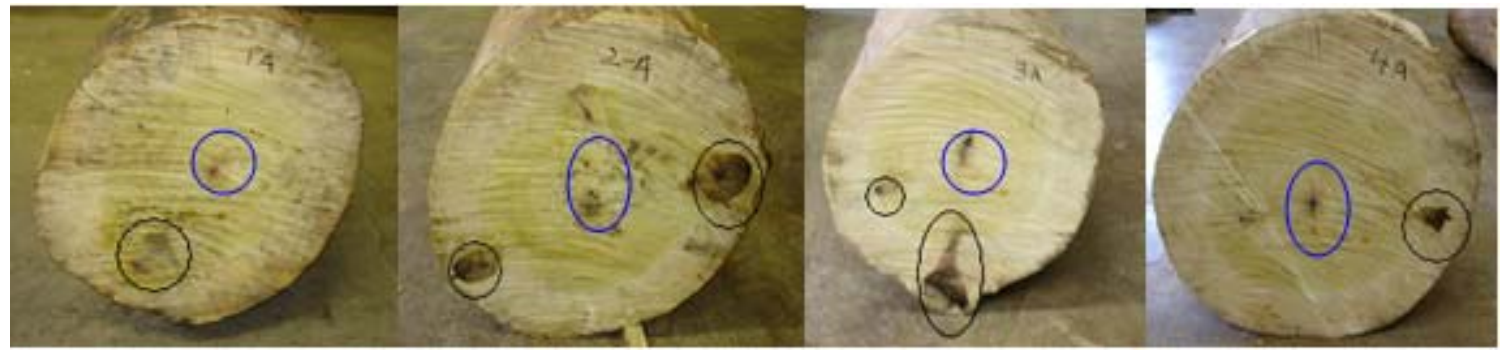
(a) Defect 1
(b) Defect 2
(c) Defect 3
d) Defect 4

(Blue circles indicate rot initiation and Black circles indicate knots)

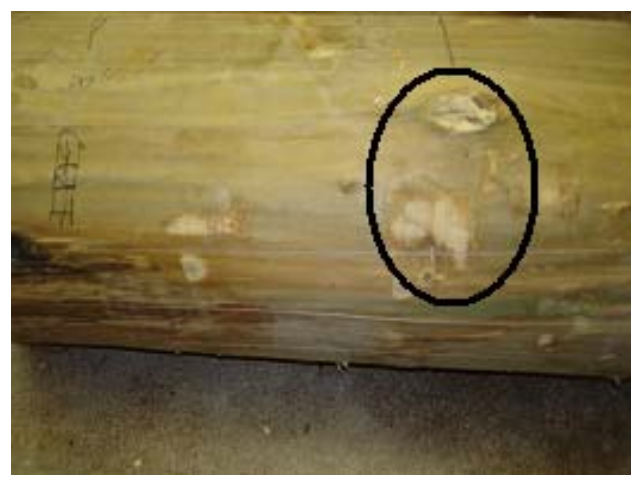

(e) Visible external knot

Figure 5-6 Cut section profile of $\log 1$ indicating presence of defects in $\log 1$ 


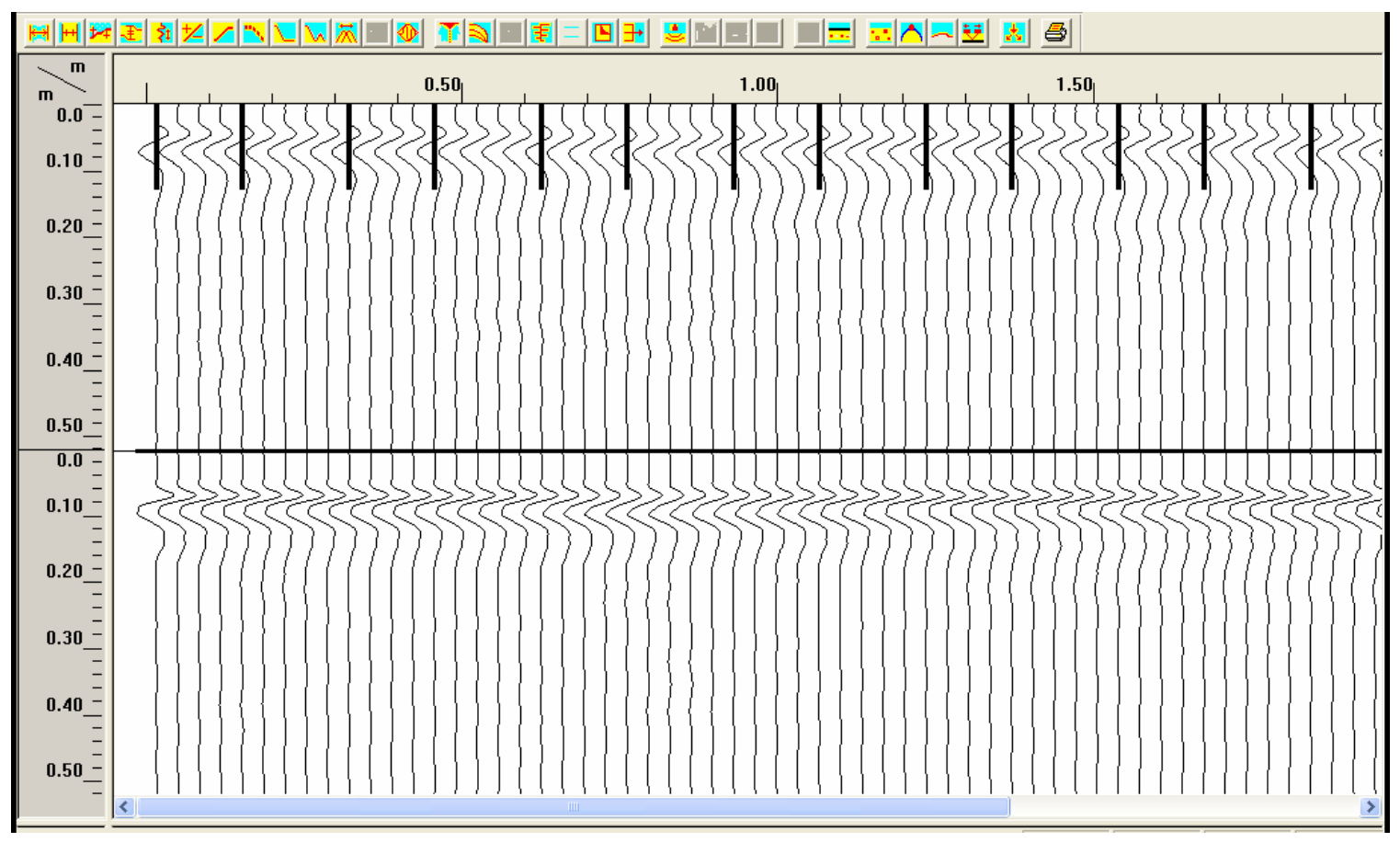

(a)

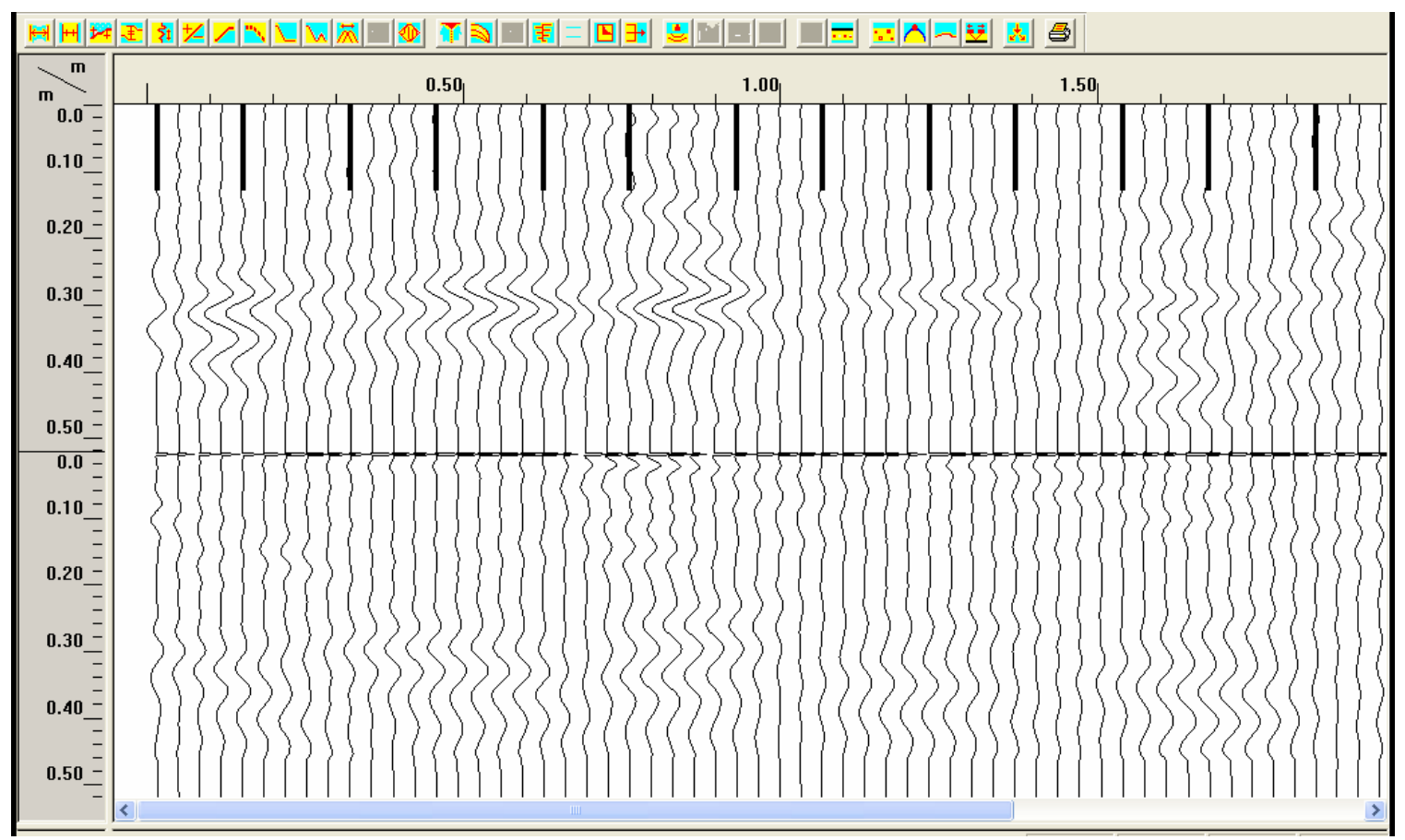

(b)

Figure 5-7 : Wiggle plot of $\log 1$, horizontal scale indicating travel distance along the length of the $\log (\mathrm{m})$ and vertical scale indicating the depth $(\mathrm{m})$, for $(\mathrm{a})$ raw data, and (b) processed data 


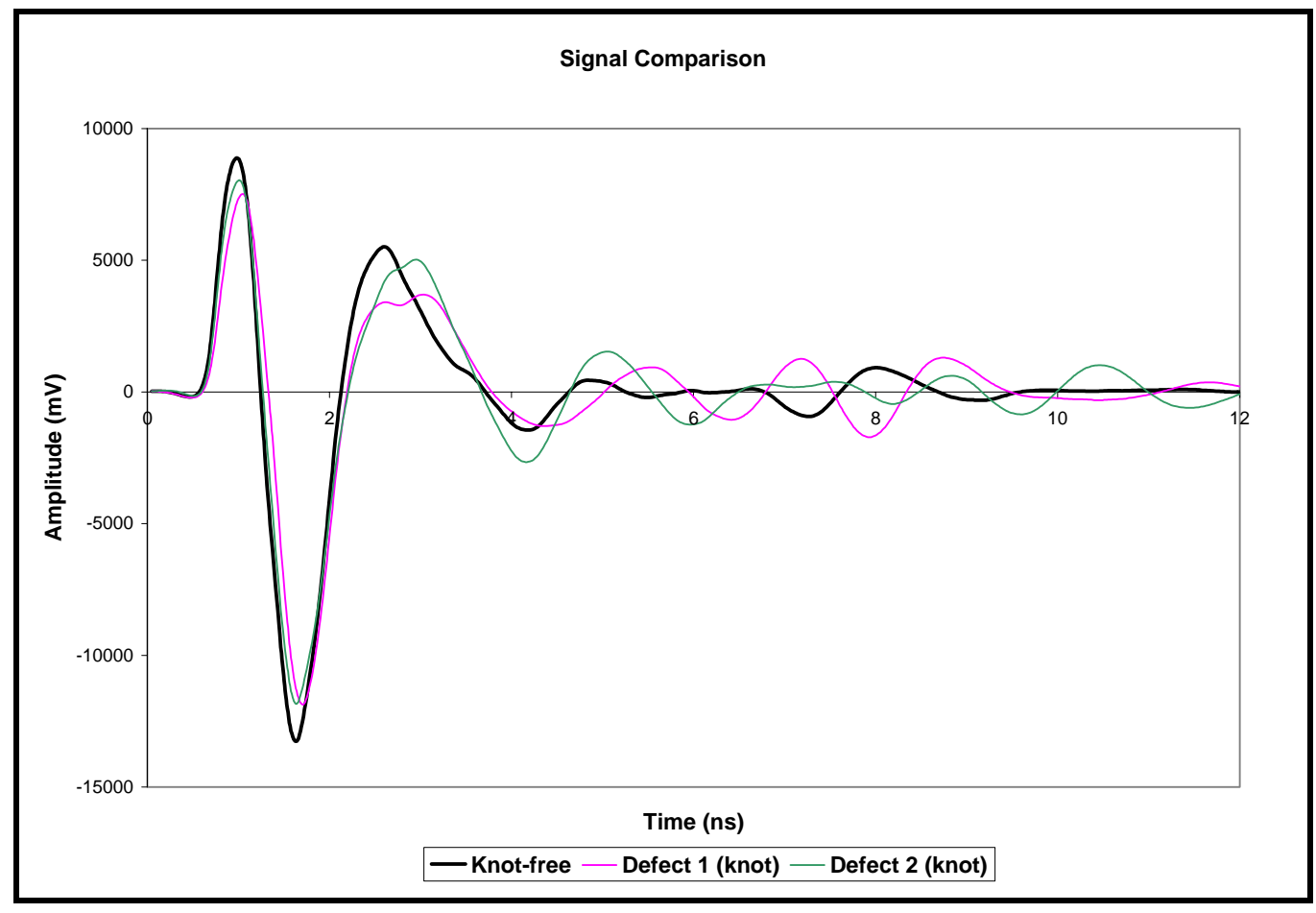

(a)

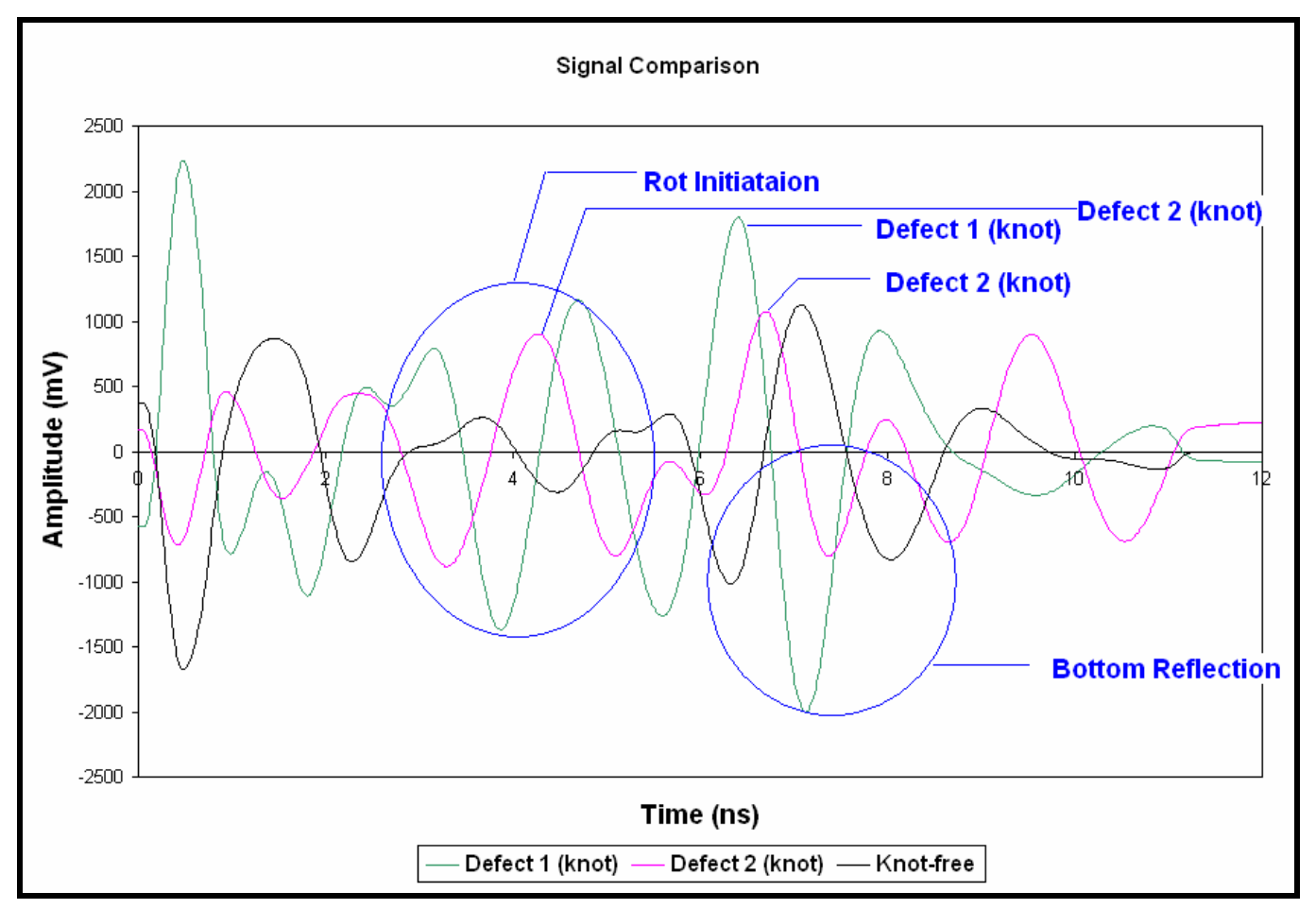

(b) 


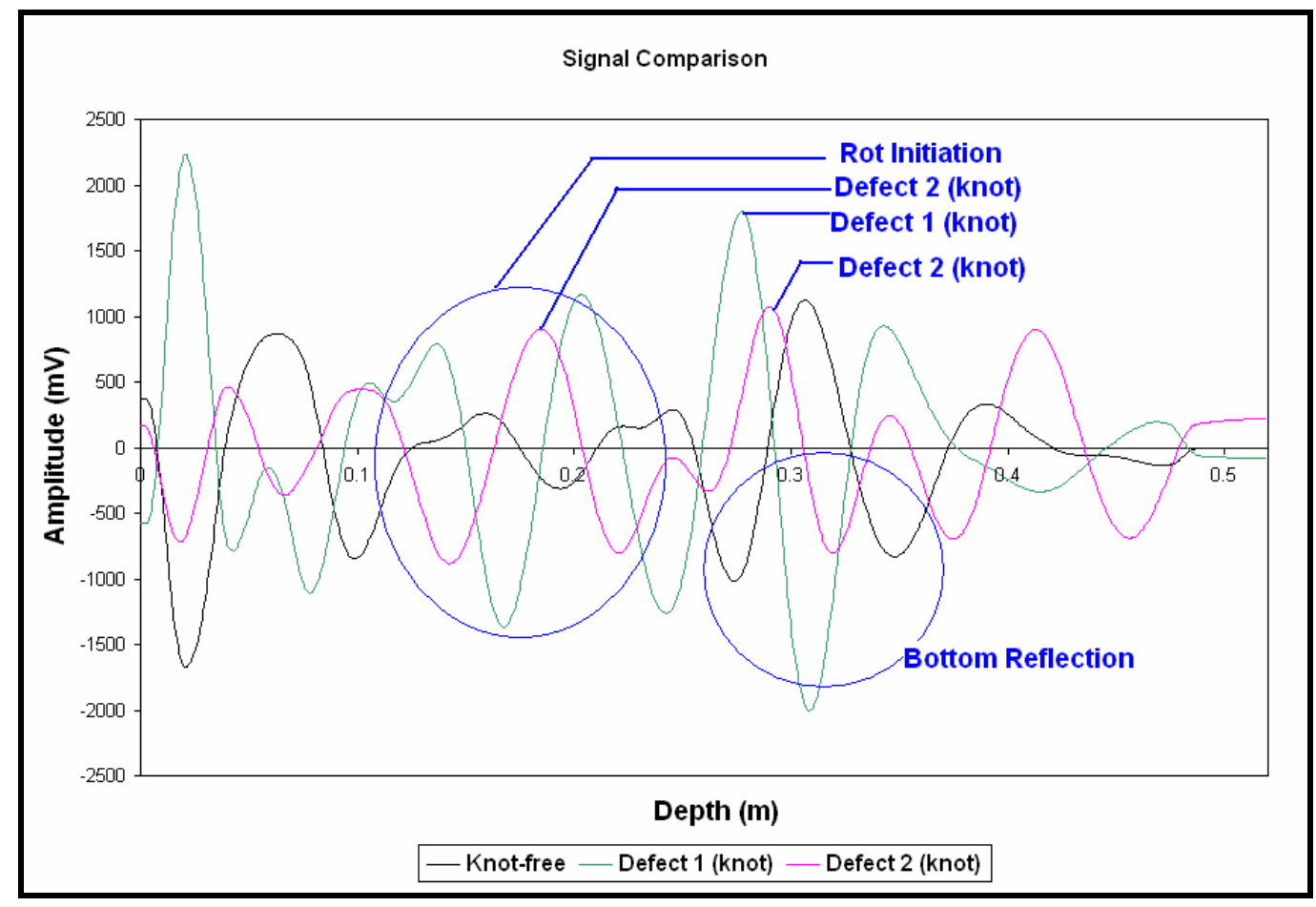

(c)

Figure 5-8 Comparison of GPR waveforms from sections with and without defects (knots) in Log 1 for (a) raw data (Amplitude vs. Time), (b) processed data (Amplitude vs. Time), and (c) processed data (Amplitude vs. Depth)

\subsection{LOG 2}

Table 5-2 Characteristics of $\log 2$

\begin{tabular}{|c|c|c|c|c|c|c|c|c|}
\hline \multirow{2}{*}{$\begin{array}{c}\text { Log } \\
\text { no. }\end{array}$} & Species & $\begin{array}{c}\text { Length } \\
(\mathbf{m})\end{array}$ & $\begin{array}{c}\text { Dutt } \\
\text { end }\end{array}$ & $\begin{array}{c}\text { Small } \\
\text { end }\end{array}$ & Surface & Max. & Average & Dielectric \\
Constant
\end{tabular}

Pictures of $\log 2$ and the GPR scanning configuration are shown in Figure 5-9. The log was quite tapered with the characteristics as given in Table 5-2. A metal rod of 
$0.016 \mathrm{~m} \mathrm{(5/8} \mathrm{in)} \mathrm{diameter} \mathrm{and} 0.1 \mathrm{~m}$ (4 in) length was inserted at a distance of $1.2 \mathrm{~m}$ from the butt end $(0.7 \mathrm{~m}$ from the small end). The position of metal rod at a distance of $1.2 \mathrm{~m}$ from the butt end is shown in Figure 5-9(b). After insertion, the rod protruded $0.05 \mathrm{~m}$ (2 in) on either side of the log's center (Figure 5-9(b)), which was at a depth of about $0.25 \mathrm{~m}$ at this location.

Figure 5-9(b) shows numbers which indicate the positions around the log for which the GPR scans were collected. The antenna configuration used for collecting the data is shown in Figure 5-9 (c). A single antenna was used for collecting the data and total of 8 GPR scans were conducted by rotating the log and bringing each marked position next to the antenna. Each scan was conducted by moving the set up along the length of the log from the butt end of the log to the small end for a given rotational position of the log with respect to the antenna. Figure 5-10 shows the data collection and processing parameters used for all the scans. These parameters were explained in Sections 4.1 and 4.2. Scans along marks 4 and 5 were selected for demonstrating the results of from $\log 2$. Other scans did not provide clear internal characteristics of defects as compared to scan along mark 4 and 5 , as discussed later.

The process of data analysis for this $\log$ was similar to that for $\log 1$. The raw GPR data is shown in Figure 5-11 for the scan along mark 4. The top set of white-blackwhite band (high amplitude signal) is due to surface coupling (antenna to log coupling). Due to high signal attenuation and reflection/transmission losses, the detection of internal features from the raw data was difficult. Therefore, the raw data was processed using the parameters shown in Figure 5-10(b), and the processed scans are shown in Figure 5-12 for the GPR scan along mark 4.

The internal defects were predicted based on location of signal clutters in the processed GPR scan as shown in Figure 5-12. These signal clutters became visible only after the raw scan was processed. It can be seen from the processed data that the time and depth axis have shifted upwards in comparison to Figure 5-11 because of zero correction. Figure 5-12 now shows the correct depth. From the processed GPR scans (Figure 5-12), internal defects could be predicted at two locations. These defects were marked as Defect 1 and Defect 2. The location of defect could also be seen in the wiggle plots (Figure 5- 
13), but the defects are more clear in Figure 5-12. The clutter in the initial region (before Defect 1) and later region (after Defect 2) in Figures 5-12 and 5-13(b) are due to edge effects in the $\log$ and should not be misconstrued as defects. Such edge effects are observed if the scan starts (or ends) very close to the edge of the log. Defect 1 was predicted to be located at a distance of $0.35 \mathrm{~m}$ from the starting point of the scan (i.e., horizontal position from the butt end of the $\log$ ) and at a depth of $0.27 \mathrm{~m}$ from the surface of the log along the mark 4. Defect 2 was predicted to be located at a distance of $1.2 \mathrm{~m}$ from the point of start of scan and a depth of $0.2 \mathrm{~m}$. The horizontal position here refers to the center of the defect. The vertical position is the depth to the top of the defect, which is what is usually predicted from GPR scans. Also, from the processed data (Figure 5-12) it could be predicted that the extent of Defect 2 was greater than that of Defect 1 .

In addition to the above defects, Figure 5-12 also shows lower amplitude signal clutter just before Defect 2. This feature was investigated after sawing the log and will be discussed later.

Figure 5-14 shows the raw data of GPR scan along mark 5. Other then the surface reflection no internal characteristics could be predicted. The processed data of the GPR scan along mark 5 is shown in Figure 5-15. This data showed an additional internal feature (Defect 3), which had a parabolic shape that was due to the inserted metal rod. The parabolic shape generally indicates a strong reflector (in this case a metallic defect) that is of relatively small thickness and oriented perpendicular to the direction of the movement of the antenna. Defect 3 was predicted to be at a distance of 1.2 meters from the butt end and at a depth of $0.29 \mathrm{~m}$ from the surface of the log along the mark 5. As stated earlier, the actual horizontal position and depth for this defect were $1.2 \mathrm{~m}$ and 0.25 $\mathrm{m}$, respectively. Thus, the depth resolution was $0.04 \mathrm{~m}$ in this case.

It should be noted that Figure 5-15 also shows Defect 1 but not Defect 2 that was seen in Figure 5-12. On the other hand, Defect 3 was seen in Figure 5-15 but not Figure 5-12. This indicates the importance of taking multiple scans around the log.

The wiggle plots shown in Figure 5-16 did not provide any additional information and were rather confusing. Therefore, they were not analyzed any further. 
Figures 5-17 and 5-18 show the processed GPR scans along marks 2 and 8, which represent two other scanning positions around the log as shown in Figure 5-9(b). These scans do not provide as much information as in the previous scans (along marks 4 and 5). Defect 1 is the only defect which showed up in these scans.

To validate the above predictions for subsurface defects, the log was sawn using a portable saw mill as follows. The log was first rotated so that mark 4 was at the bottom. Then it was sawed to remove the top portion and make it flat (Figure 5-19(a)). It was then inverted so that mark 4 was at the top (Figure 5-19(b)). Then the top part removed to make it flat. The resulting sawn $\log$ is shown in Figure 5-20. This log was then sliced into several flat horizontal layers (approximately 1 " or $0.025 \mathrm{~m}$ thick).

To validate the predictions, the GPR depth scan along mark 4 (Figure 5-12(b)) was reproduced in Figure 5-21 and compared with a horizontal slice showing the actual defects. It is now obvious that Defects 1 and 2 in the GPR scan represented subsurface knots. Also, Figure 5-21 shows that Defect 2 (knot) is bigger compared to Defect 1 (knot). The actual depth of Defect 1 was measured to be $0.26 \mathrm{~m}$ and was at a distance of $0.4 \mathrm{~m}$ from the butt end, which compared well with the previously mentioned GPR prediction of $0.27 \mathrm{~m}$ and $0.35 \mathrm{~m}$, respectively. Similarly, the measured depth of Defect 2 was $0.22 \mathrm{~m}$ and was at a distance of $1.2 \mathrm{~m}$ from the butt end, which compared very well with GPR's predicted depth of $0.2 \mathrm{~m}$ at $1.2 \mathrm{~m}$ distance. Also, Figure 5-21 revealed that the signal clutter observed between Defects 1 and 2 in the GPR scan (that showed weaker reflection compared to the defects) represented change in grain direction within the log.

As the log was sawn further, Defect 3, which appeared in the GPR scan along mark 5, became obvious. The processed GPR data (Figure 5-15) and the corresponding metal defect (Defect 3) are shown in Figure 5-22. The metal defect inserted in the log is shown in Figures 5-22 and 5-23. The actual depth of Defect 3 was measured to be 0.25 $\mathrm{m}$, while the GPR scan had predicted a depth of $0.28 \mathrm{~m}$. This defect was located at a distance of $1.2 \mathrm{~m}$, which was accurately predicted by GPR as $1.2 \mathrm{~m}$. From the actual measured depths, it was seen that Defect 3 was located $0.03 \mathrm{~m}$ below Defect 2 along scan 4 and 5. In the GPR scan along mark 4, Defect 2 was prominent, and this obscured the reflection from Defect 3, making it invisible. In the mark 5 GPR scan, Defect 2 did not 
produce a prominent reflection, and thus Defect 3 could be seen clearly. Therefore, it is necessary to scan the log from multiple positions around it, and combine all the defect observations to achieve a complete picture of the subsurface defects.

From the processed GPR scans, the individual waveforms for defective sections were extracted and compared with a waveform from a defect-free section. The waveform comparisons for GPR scans along mark 4 and mark 5 are shown in Figure 5-24 and Figure 5-25.

The signal compassion for defective and defective areas corresponding to GPR scan along mark 4 is shown in Figure 5-24. The vertical axis for all the waveform in Figure 5-24 shows the waveform amplitude. The horizontal axis for the raw and the processed waveforms in Figure 5-24(a) and 5-24(b) shows two-way travel time through the depth of the log. In the processed waveform in Figure 5-24(c), the horizontal axis shows the distance through the depth of the log. The raw waveforms in Figure 5-24(a) show very little differences between the signals from defective and defect-free areas. On the other hand, the processed waveforms in Figure 5-24(b) and 5-24(c) showed several key features. The initial high amplitude features between 0 and $2 \mathrm{~ns}(0$ to $0.05 \mathrm{~m})$ represents surface reflection (antenna-log interface). Defect 1 and 2 waveforms show additional prominent peaks indicating the presence and location of these defects. The positive amplitude at about $5.2 \mathrm{~ns}(0.2 \mathrm{~m})$ corresponds to Defect 2 which is a knot that was measured to be at $0.22 \mathrm{~m}$ depth based on sawing. The peak near $7 \mathrm{~ns}(0.27 \mathrm{~m})$ was due to Defect 1 (knot), whose actual measured depth was $0.26 \mathrm{~m}$.

The process of analysis of Figure 5-25 is similar to Figure 5-24 as explained in the previous paragraph. The raw waveforms in Figure 5-25(a) showed very little differences between the signals. On the other hand, the processed waveforms in Figure 525(b) and 5-25(c) showed several key features. The initial high amplitude features between 0 and 2 ns $(0$ to $0.05 \mathrm{~m}$ ) represents surface reflection. It can be seen in Figure 525(b) and 5-25 (c) that the defect-free waveform is flatter (that is, low signal amplitudes) compared to the Defect 1 and Defect 3 waveform, thus indicating no internal features. The Defect 1 and Defect 3 waveforms show additional prominent peaks indicating the 
presence and location of these defects. The peak at $7 \mathrm{~ns}(0.27 \mathrm{~m})$ in Figures 5-25(b) and (c) was due to Defect 1 (knot), whose actual measured depth was $0.26 \mathrm{~m}$.

The positive peak in Figures 5-25(b) and (c) at about $7.8 \mathrm{~ns}(0.28 \mathrm{~m})$ corresponds to Defect 3 (metal rod), whose measured depth in the log was $0.25 \mathrm{~m}$. These figures correspond to GPR scan along mark 5, which showed Defect 1 as well, but not Defect 2, as explained earlier.

The above results showed that the defects in the sawed sections correlated very well with GPR scans with a depth resolution between 0.01 to $0.03 \mathrm{~m}$. Also, the prediction of distance of the defective section from the butt end of the log (that is, distance along the length of the log) was found to be very accurate.

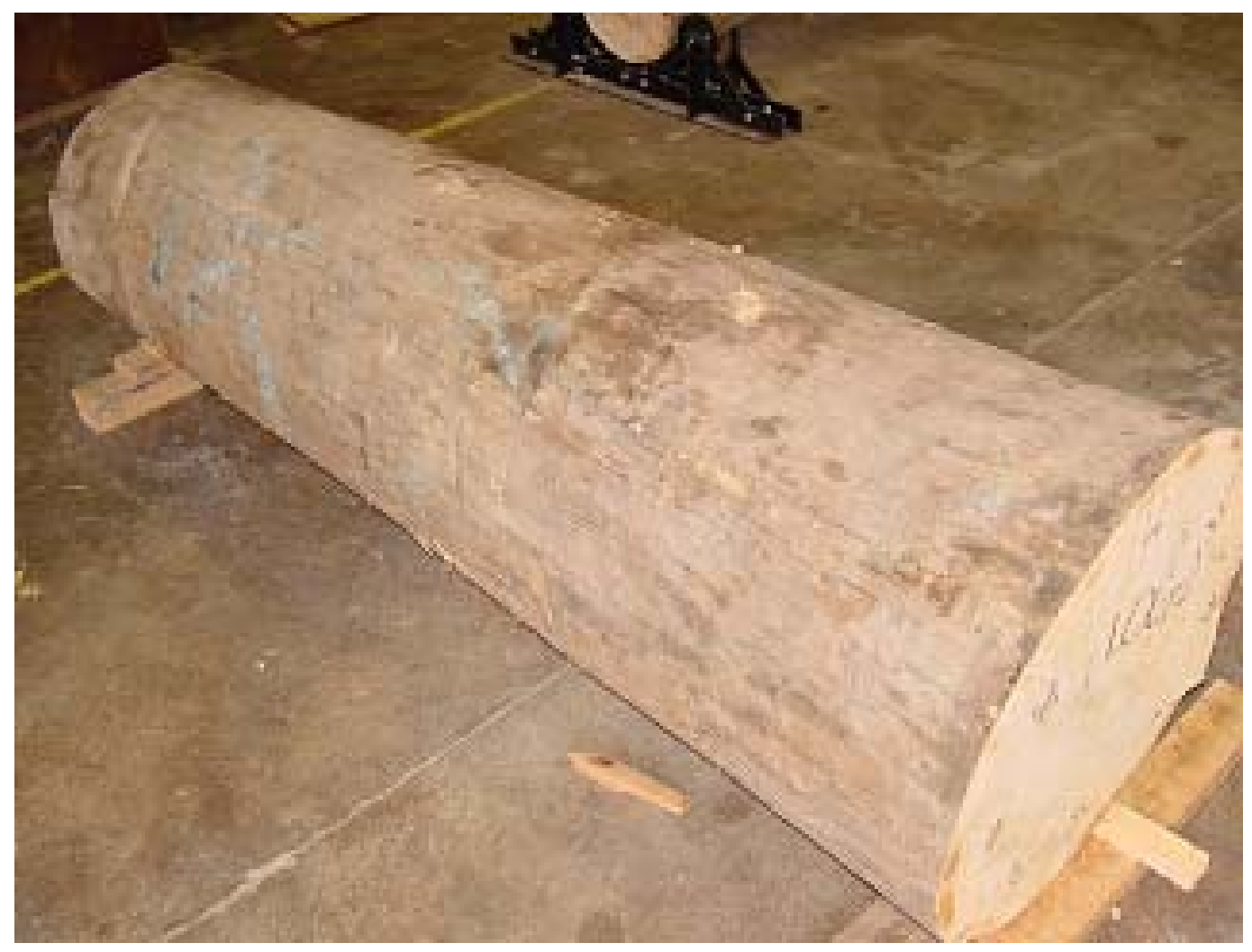

(a) 


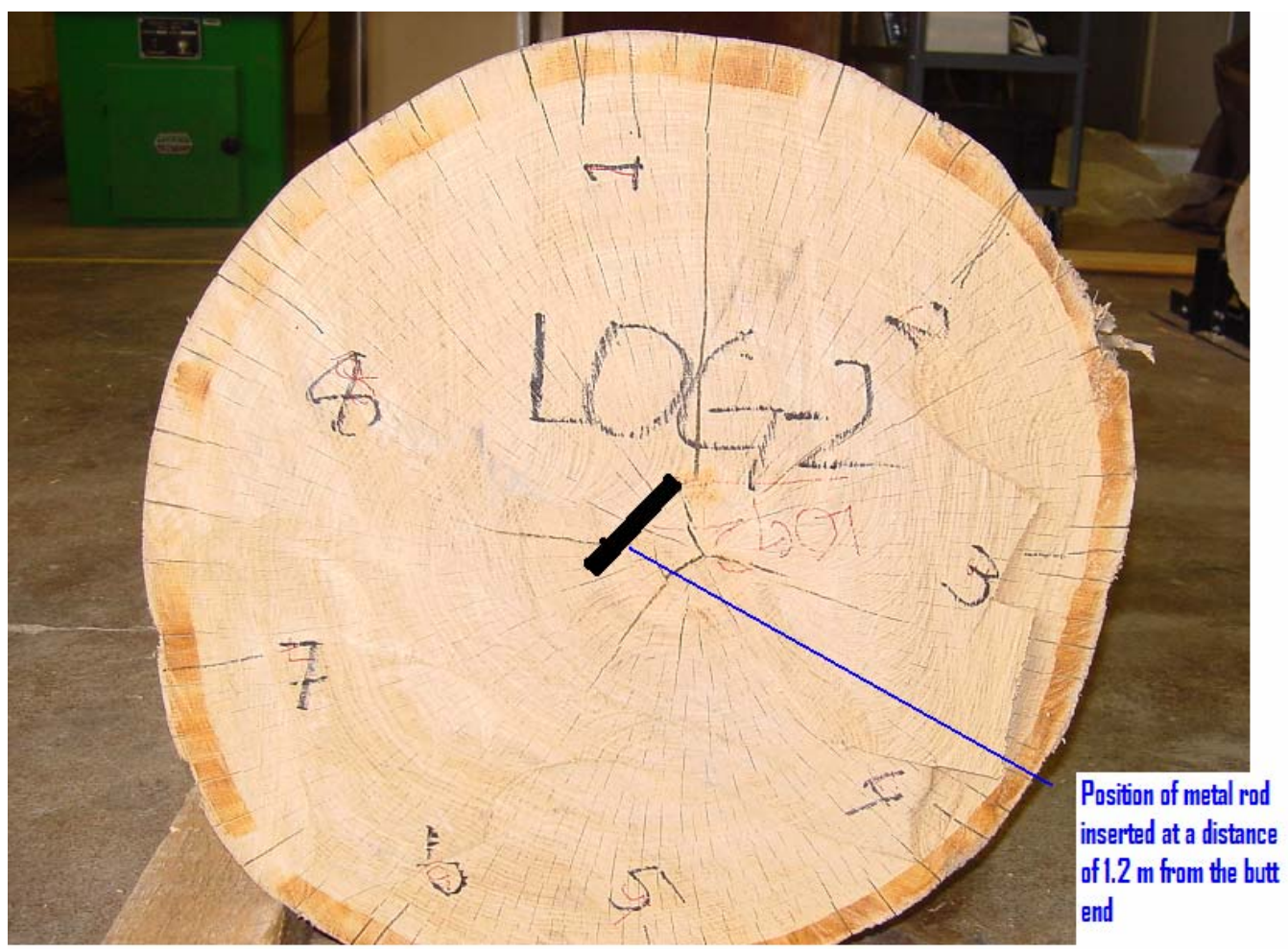

(b)

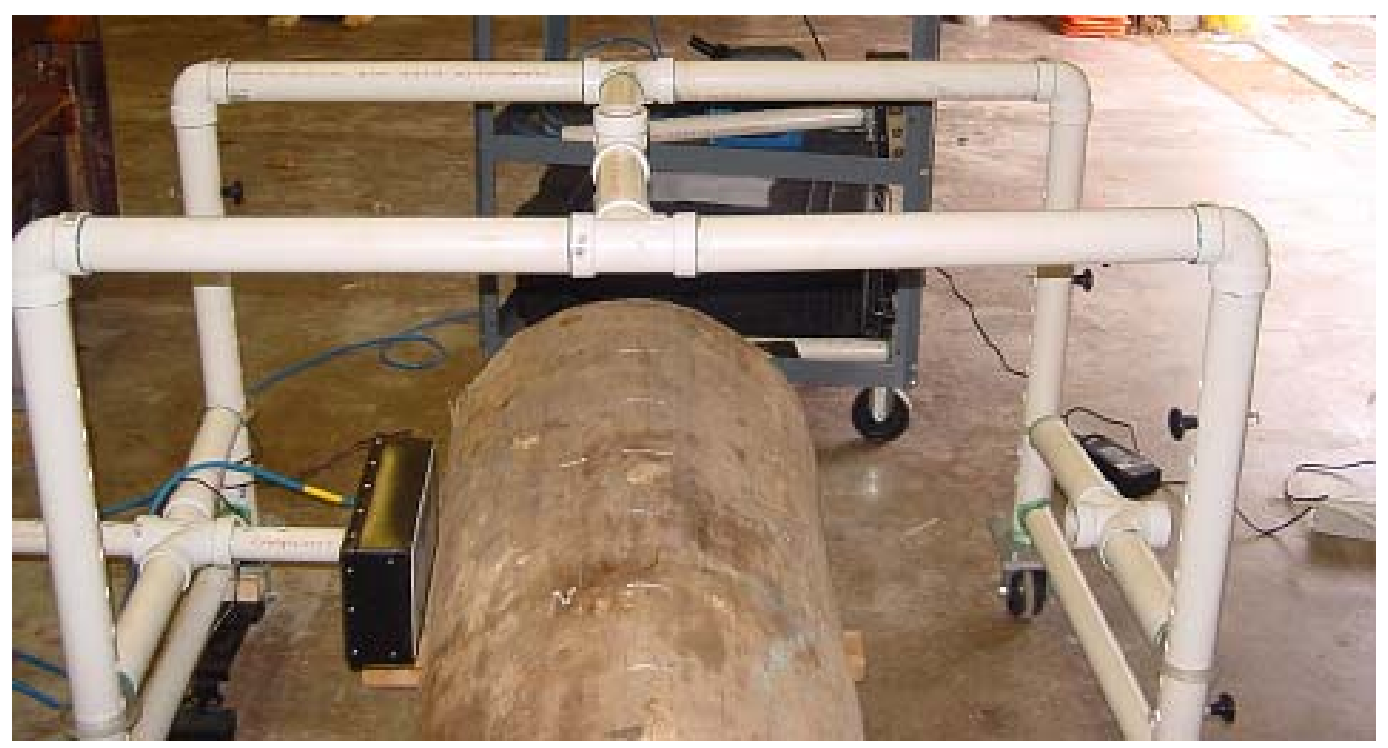

(c)

Figure 5-9 Pictures showing (a) Log 2, (b) marks indicating various GPR scanning positions around the log, and (c) antenna position 


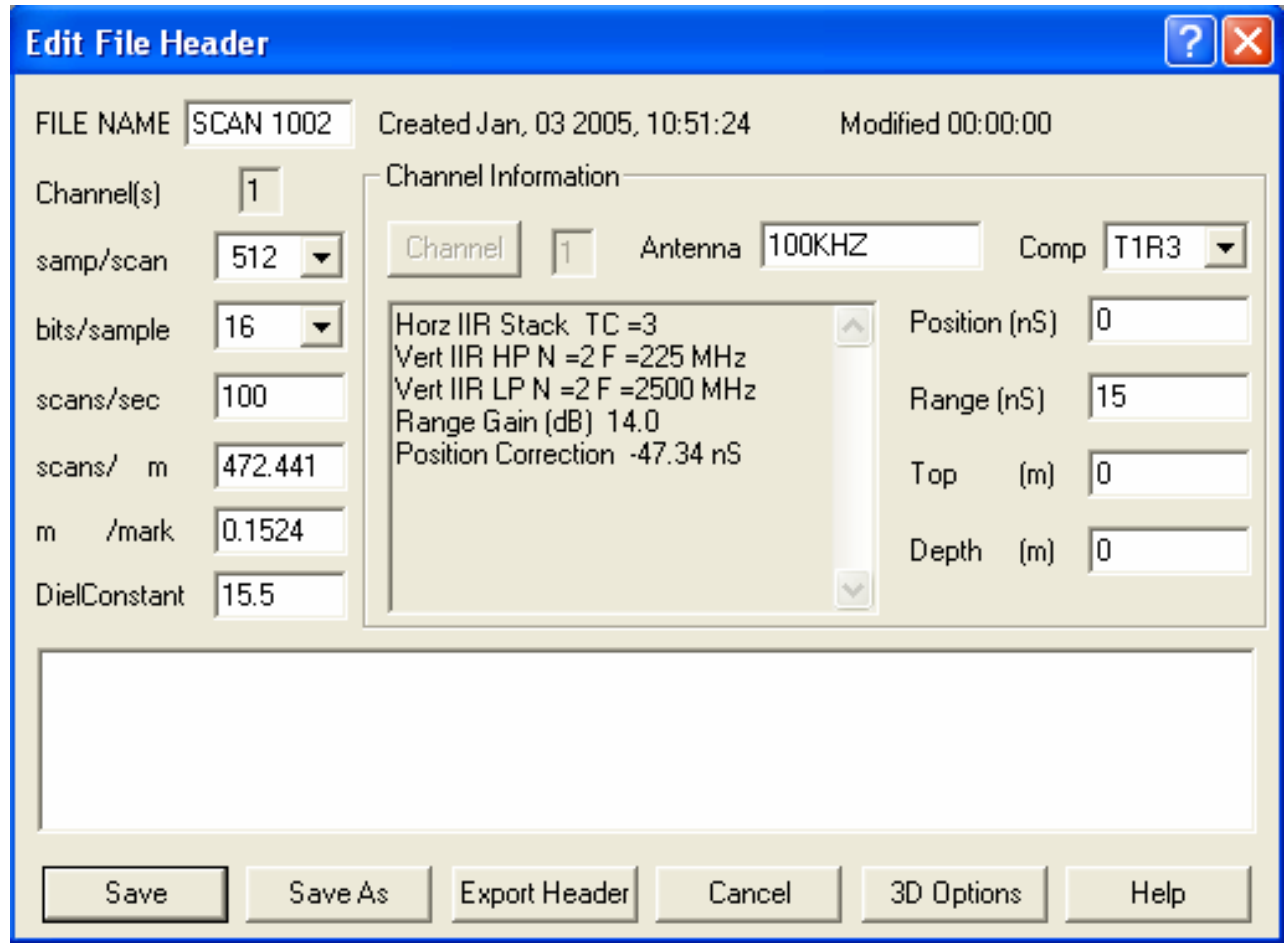

(a)

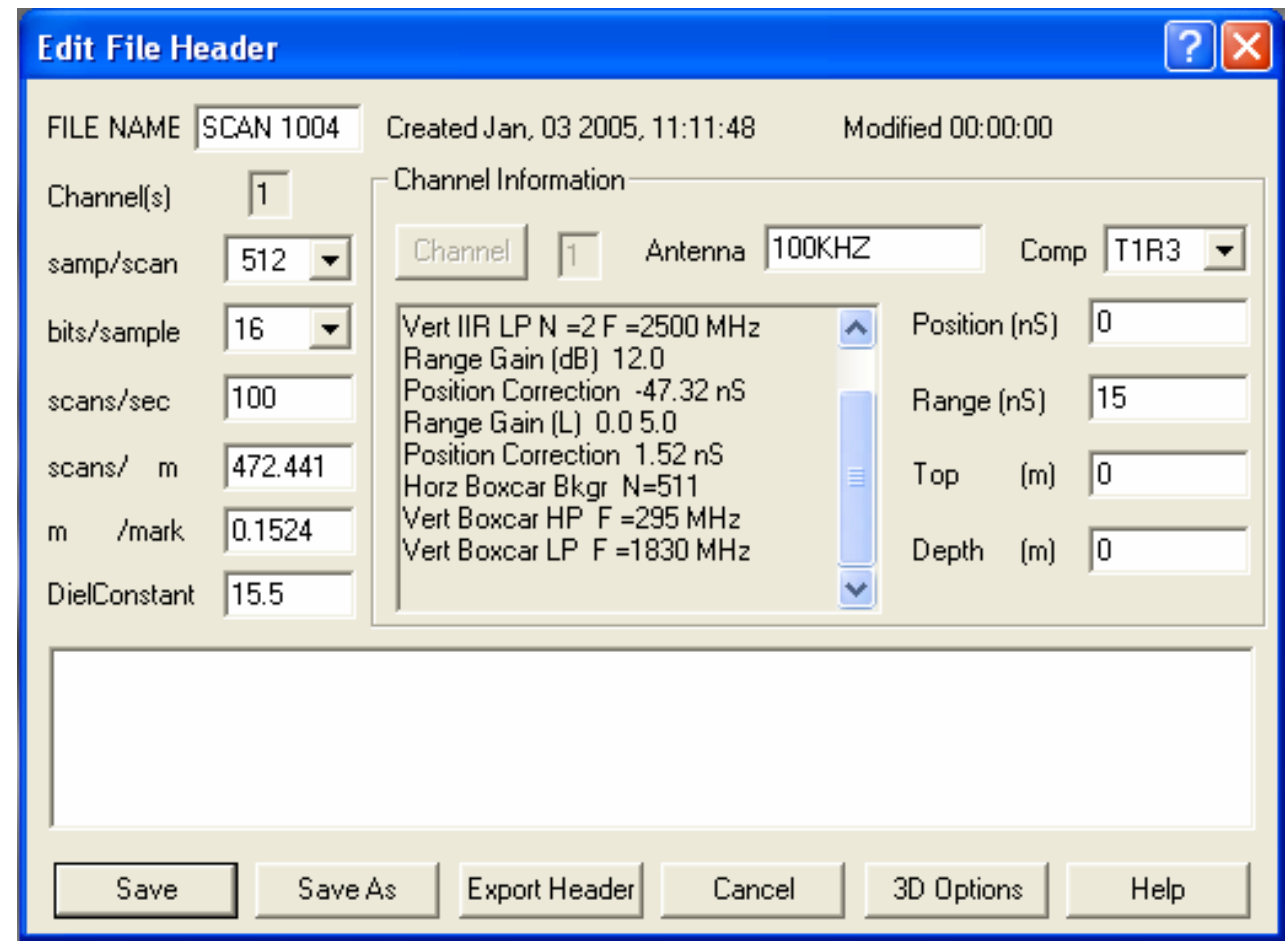

(b)

Figure 5-10 : (a) Data collection parameters (b) data processing parameters 


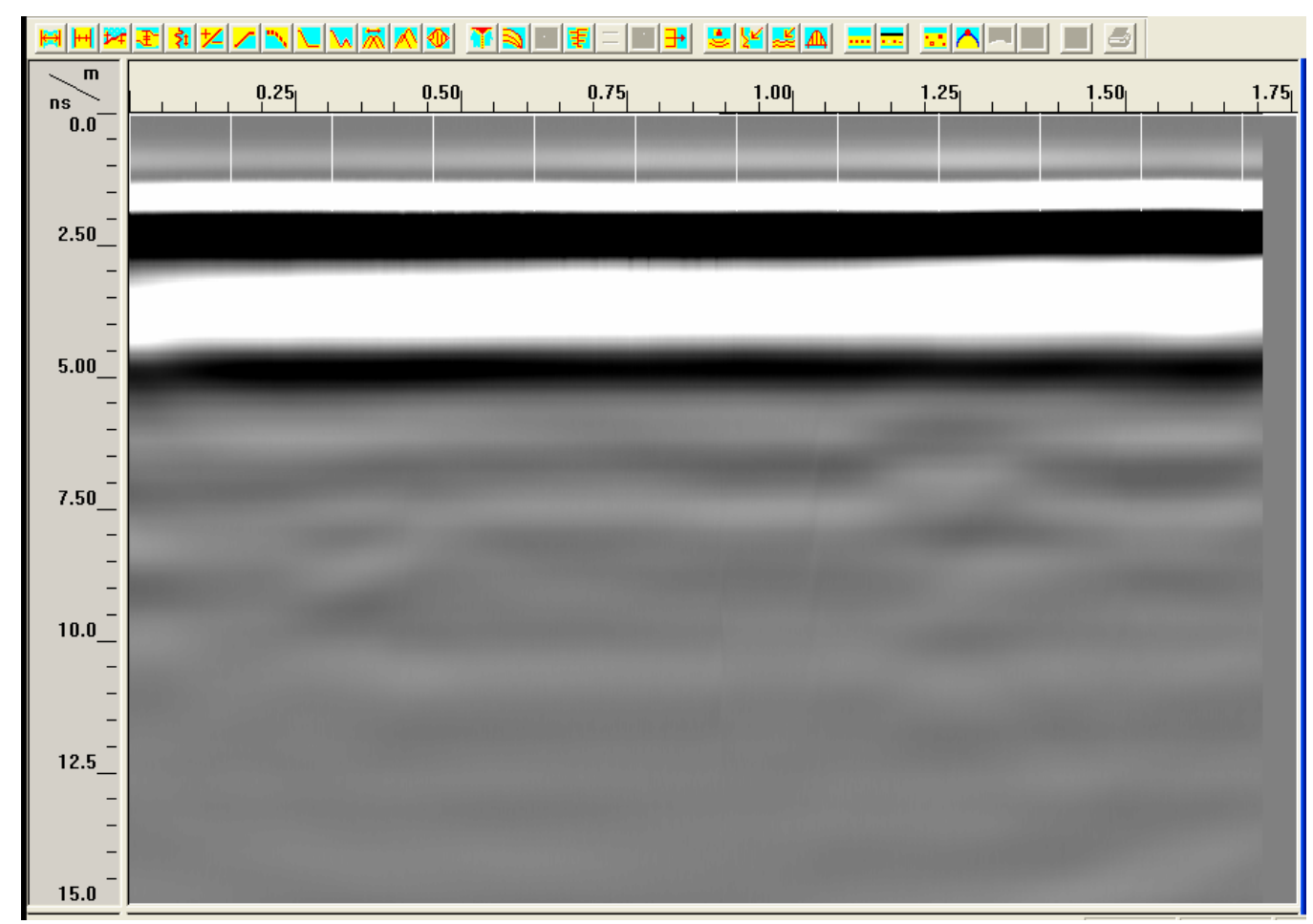

(a)

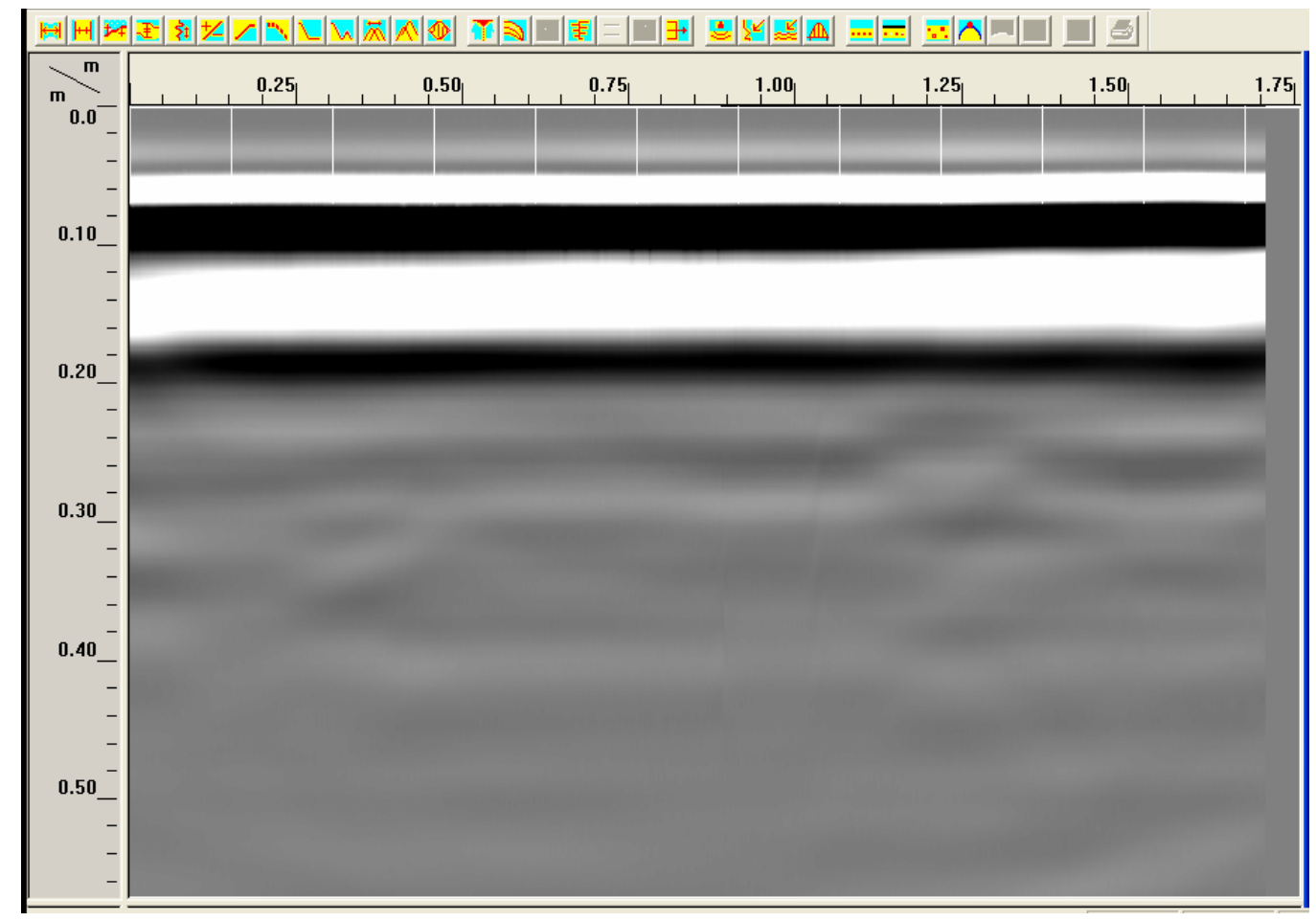

(b)

Figure 5-11 Raw GPR data of Log 2 along mark 4, horizontal scale indicating the travel distance along the length of the $\log (\mathrm{m})$ and the vertical scale indicating (a) time (ns) (b) depth (m) 


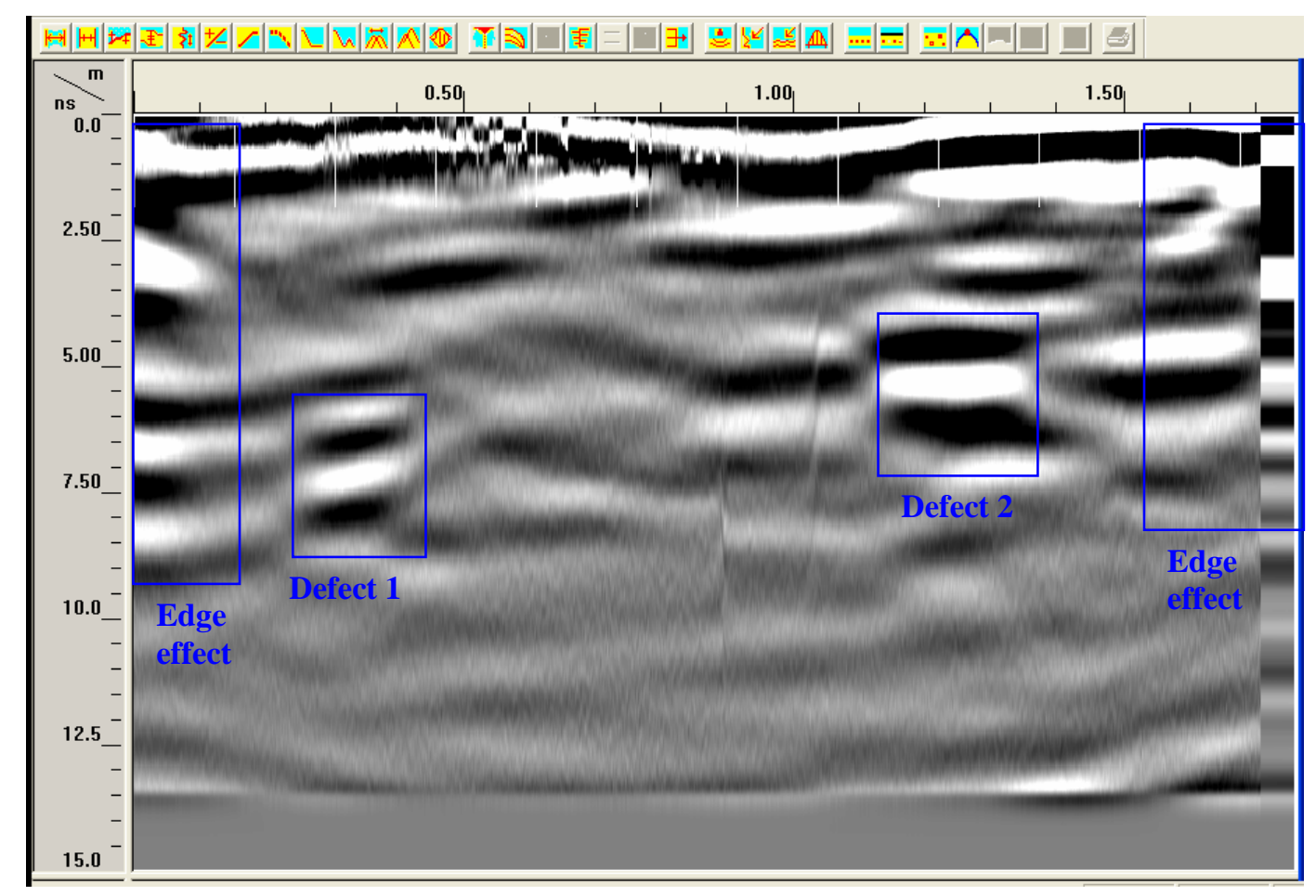

(a)

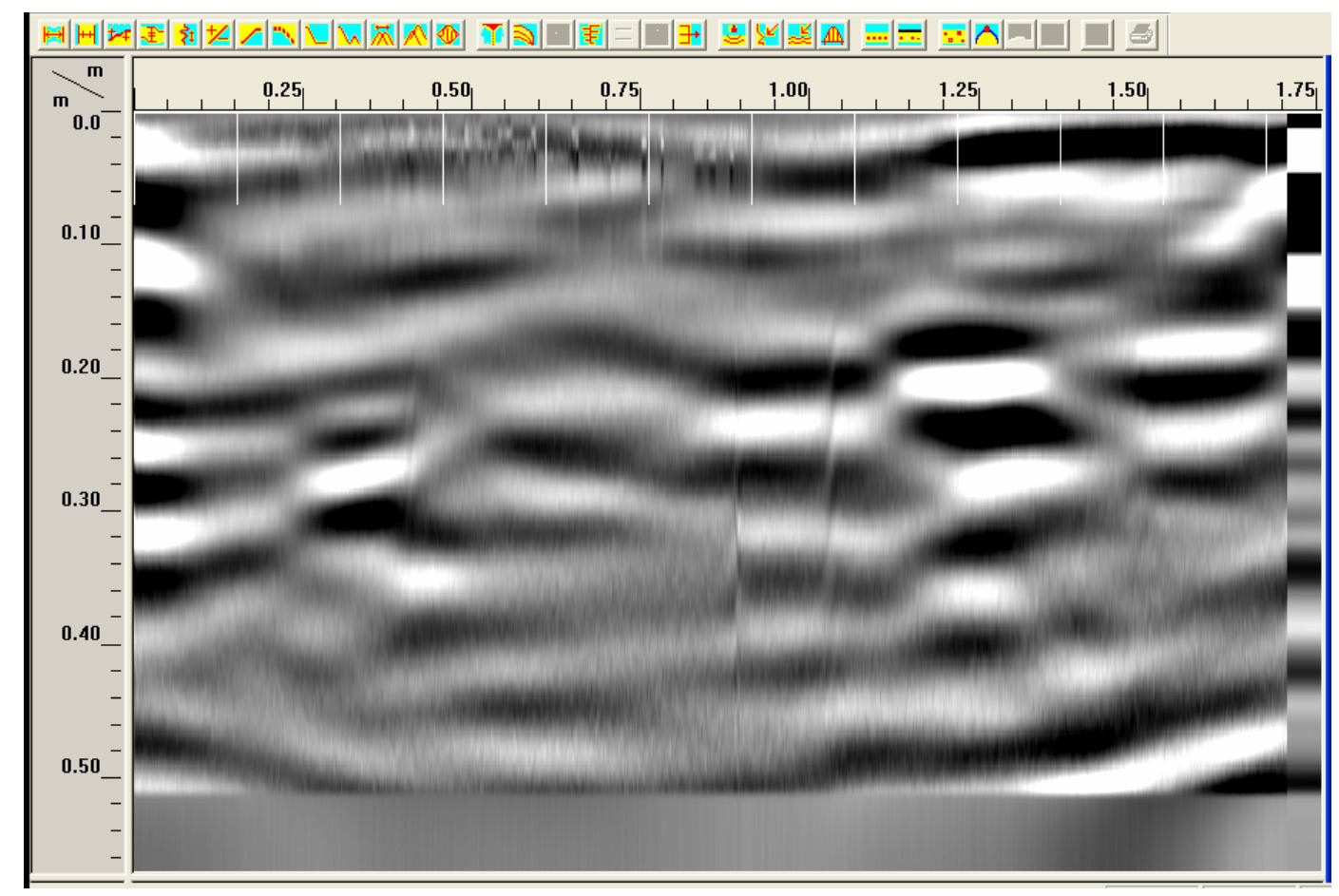

(b)

Figure 5-12 Processed GPR data of Log 2 along mark 4, horizontal scale indicating the travel distance along the length of the $\log (\mathrm{m})$ and the vertical scale indicating (a) time (ns) (b) depth (m) 


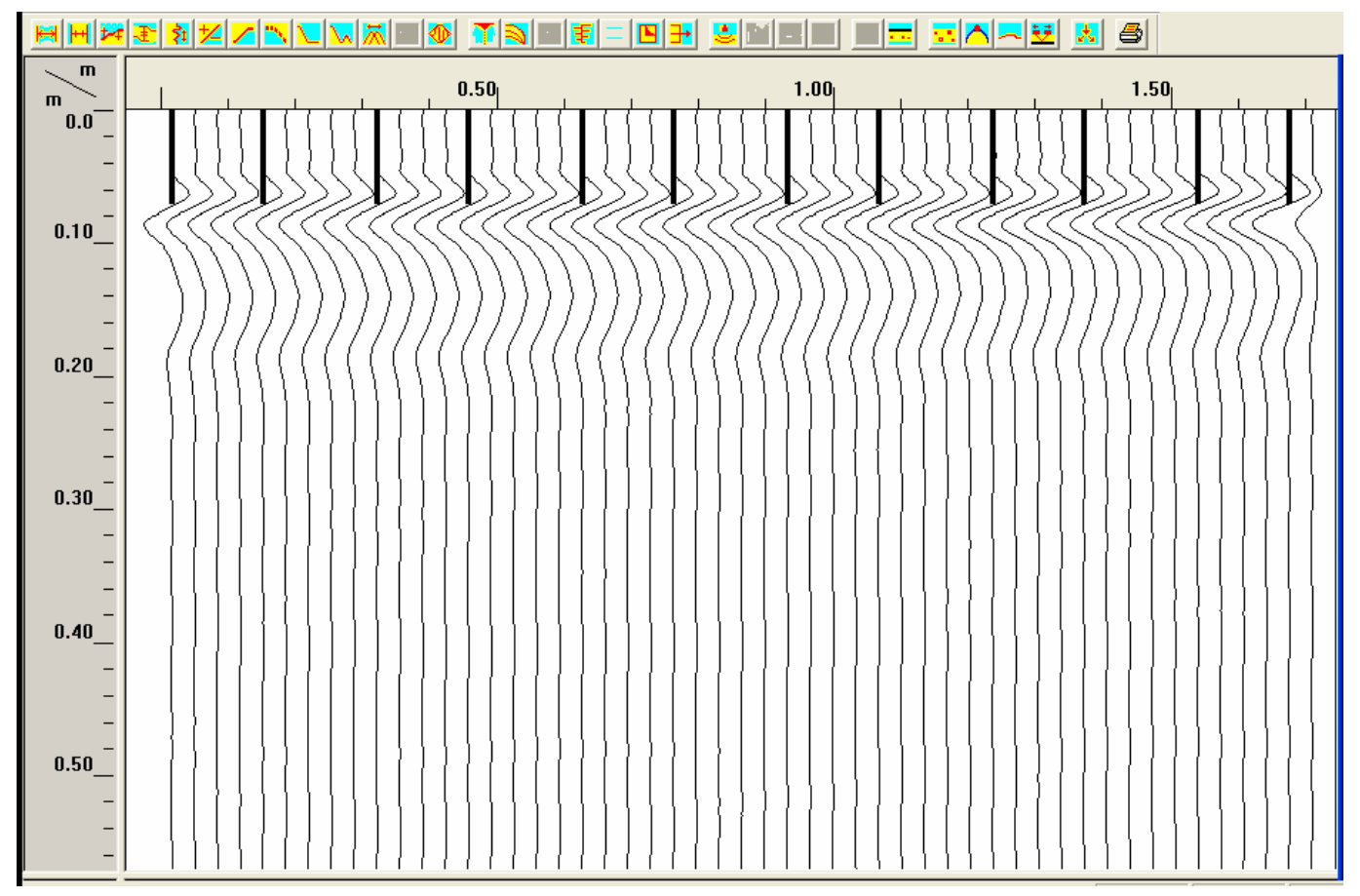

(a)

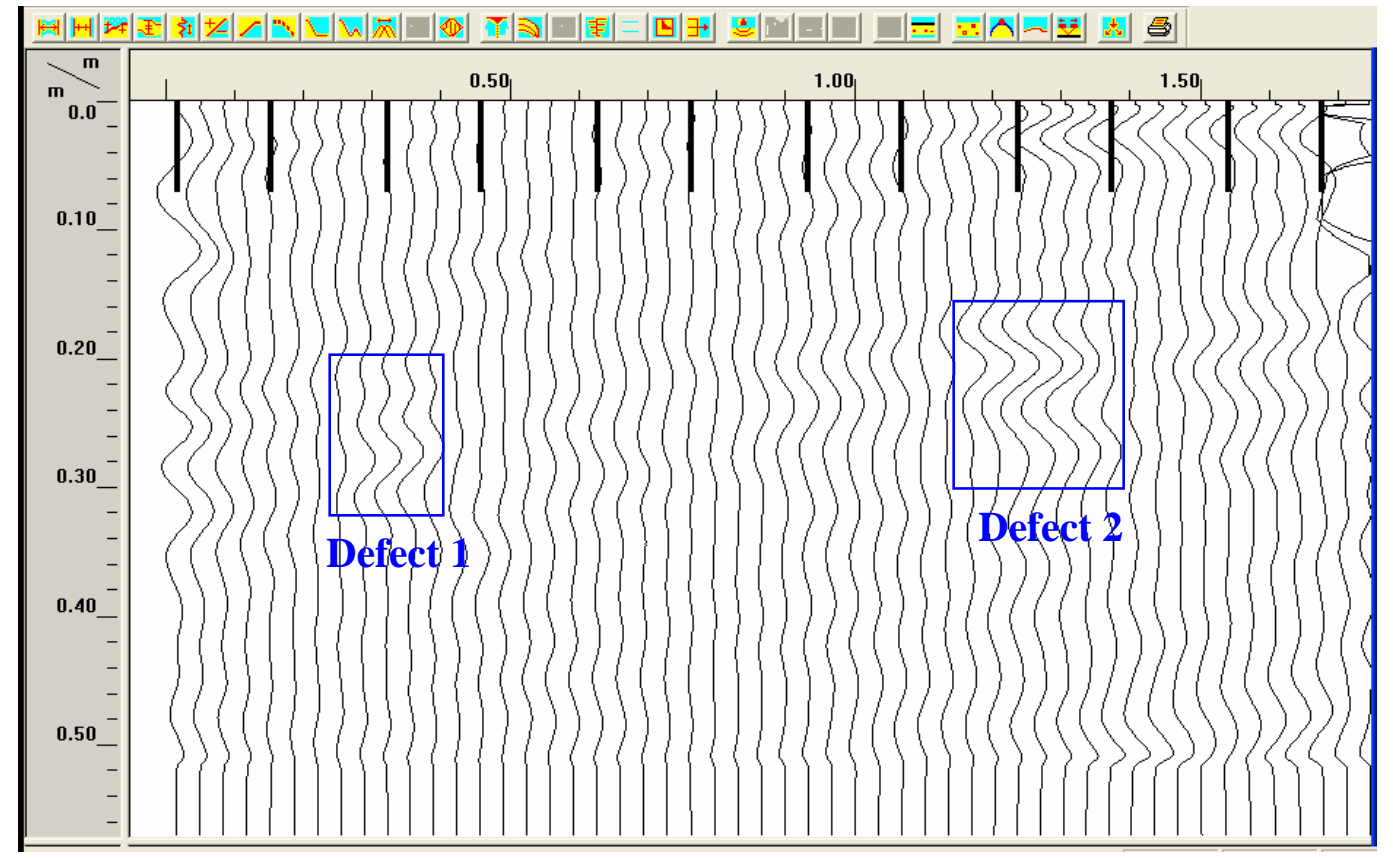

(b)

Figure 5-13 Wiggle plot of $\log 2$ along mark 4, horizontal scale indicating travel distance along the length of the $\log (\mathrm{m})$ and vertical scale indicating the depth $(\mathrm{m})$, for (a) raw data, and $(\mathrm{b})$ processed data 


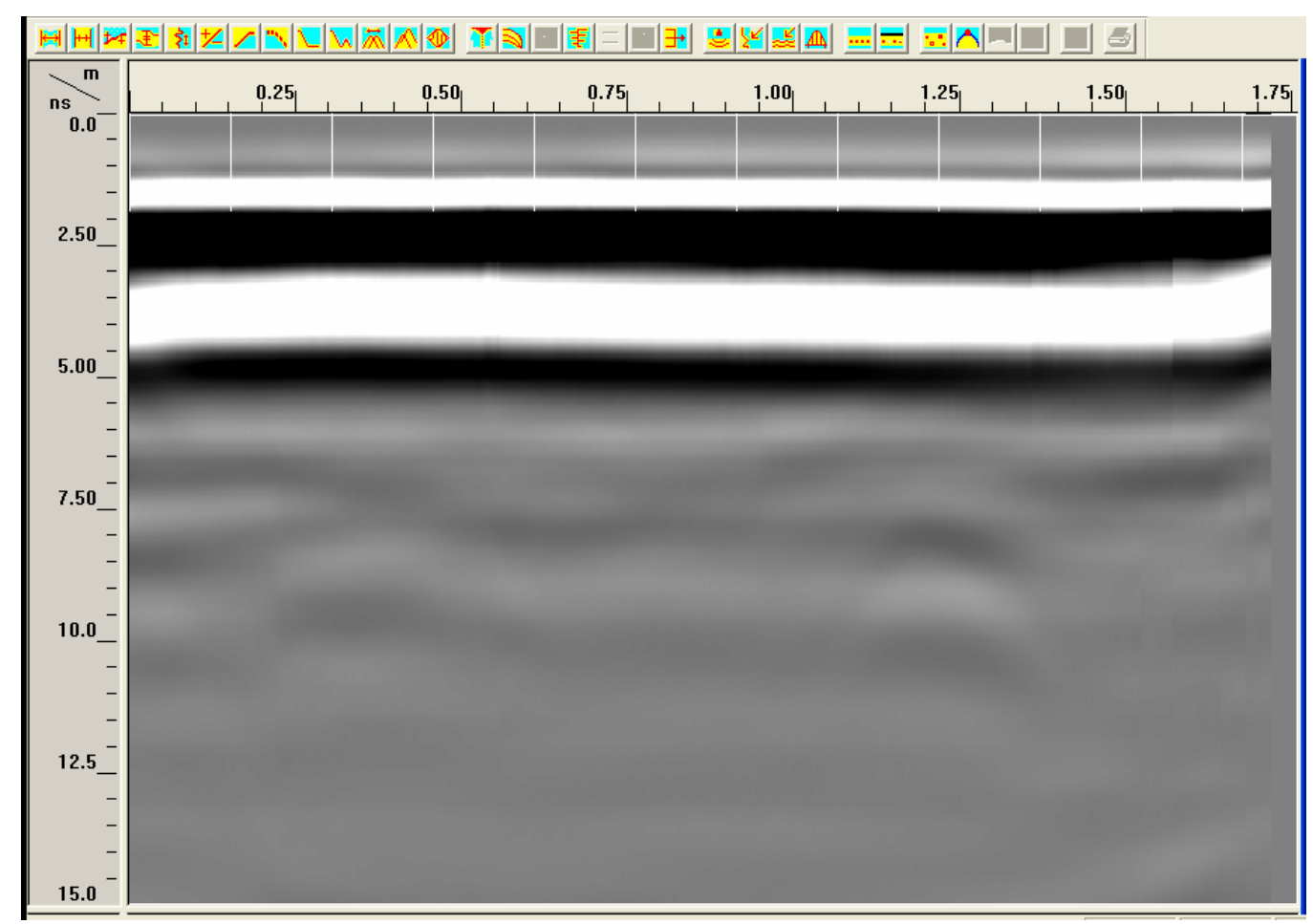

(a)

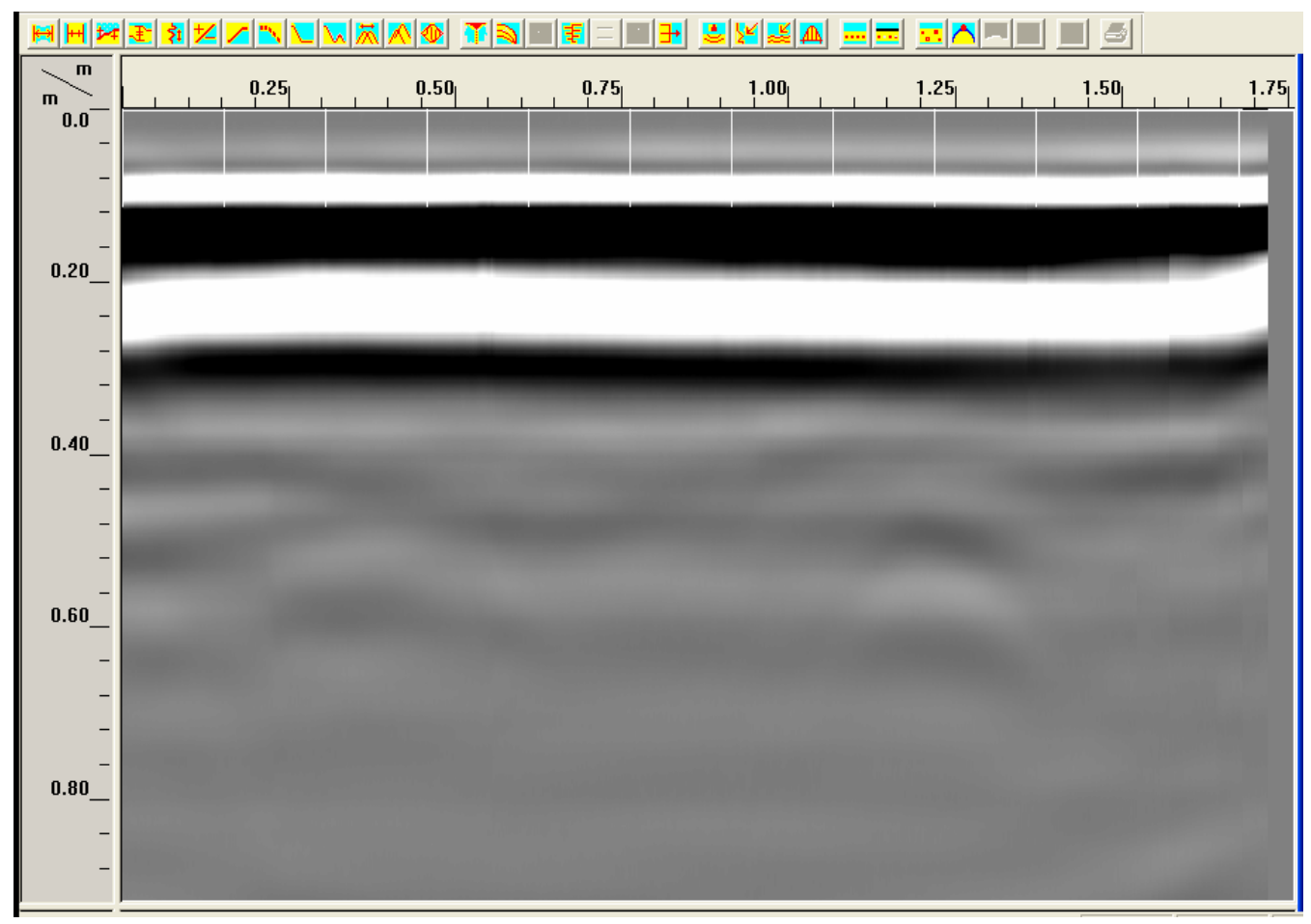

(b)

Figure 5-14 : Raw GPR data of $\log 2$ along mark 5, horizontal scale indicating the travel distance along the length of the $\log (\mathrm{m})$ and the vertical scale indicating (a) time (ns) (b) depth (m) 


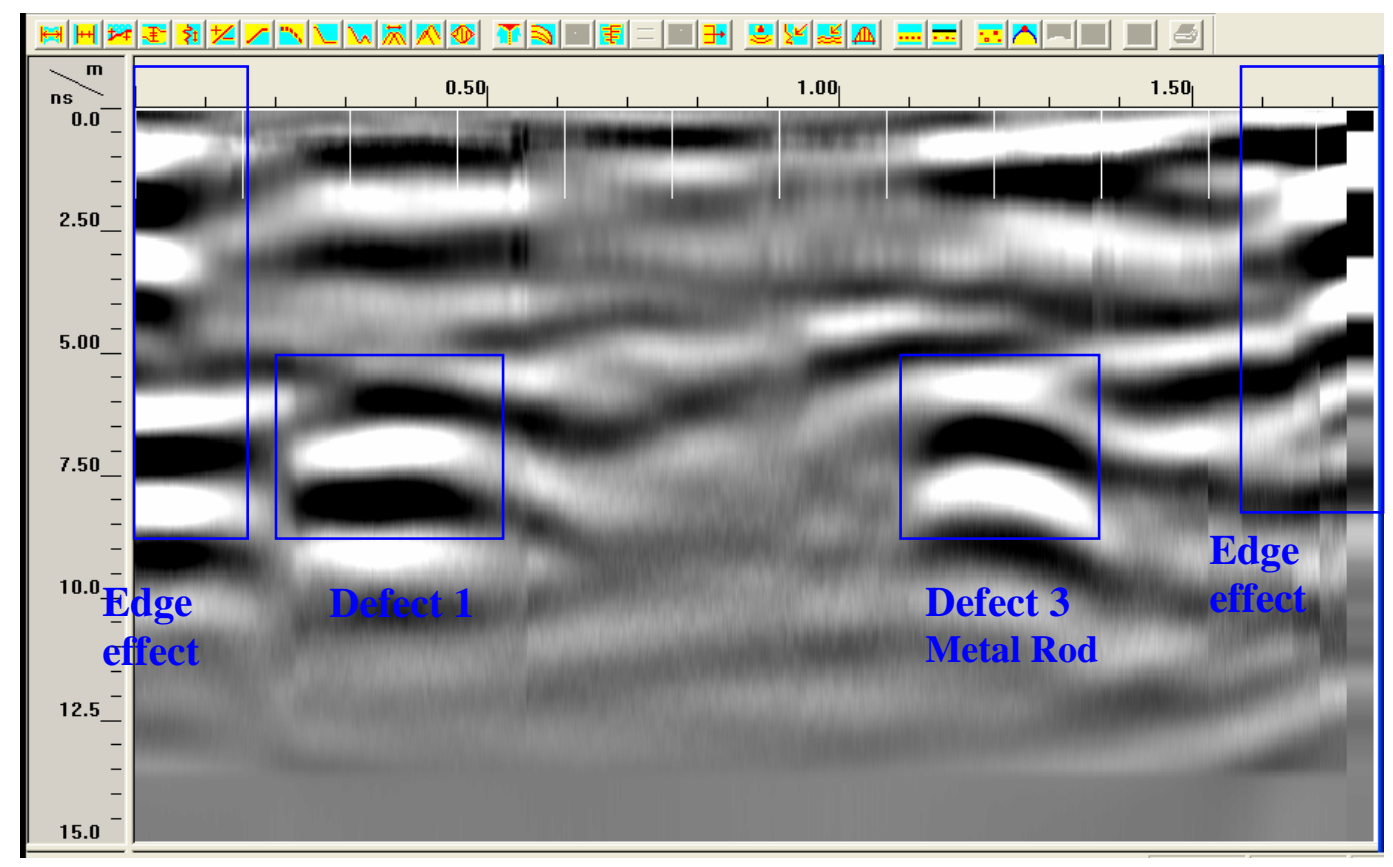

(a)

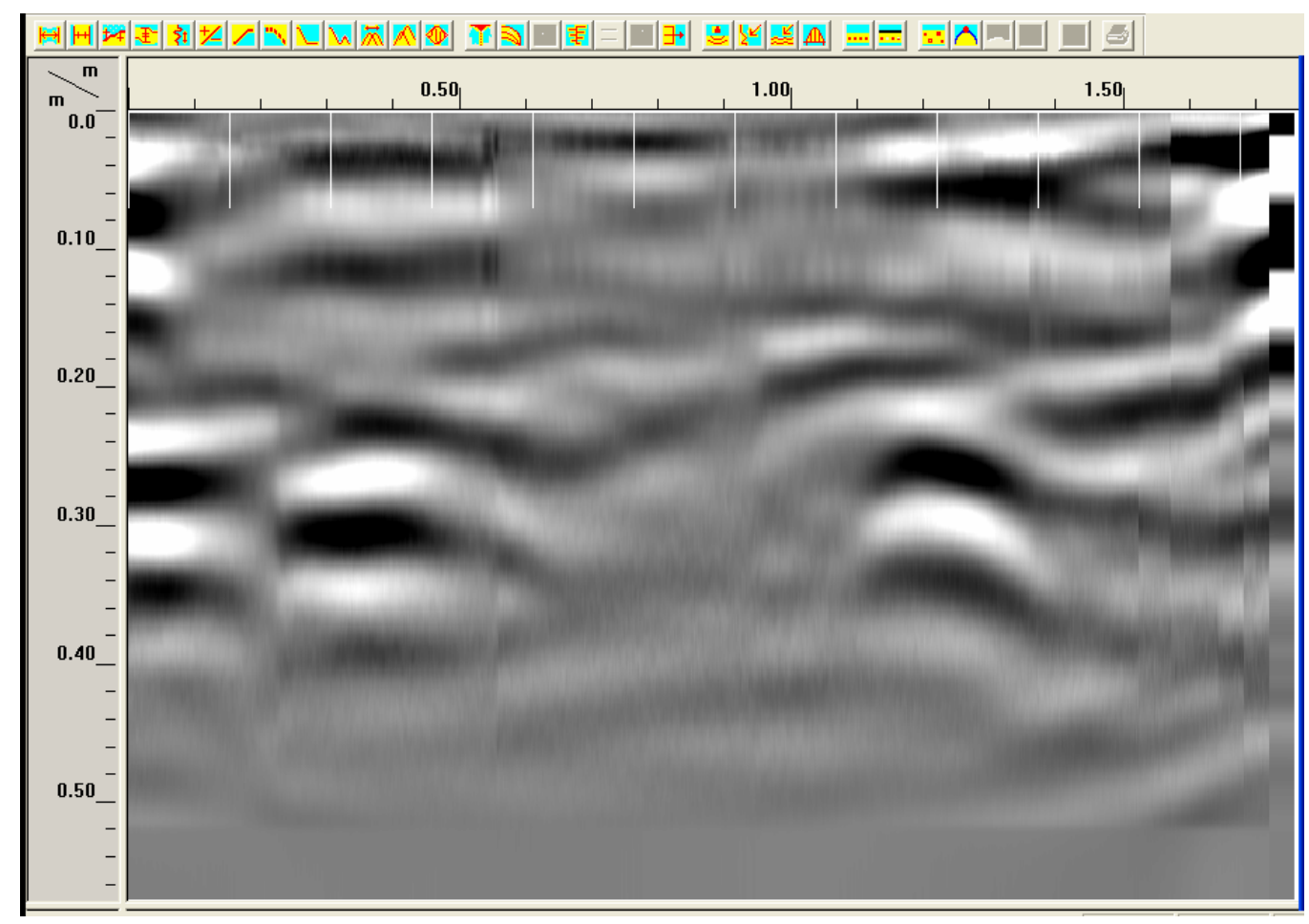

(b)

Figure 5-15 Processed GPR data of Log 2 along mark 5, horizontal scale indicating the travel distance along the length of the $\log (\mathrm{m})$ and the vertical scale indicating (a) time (ns) (b) depth (m) 


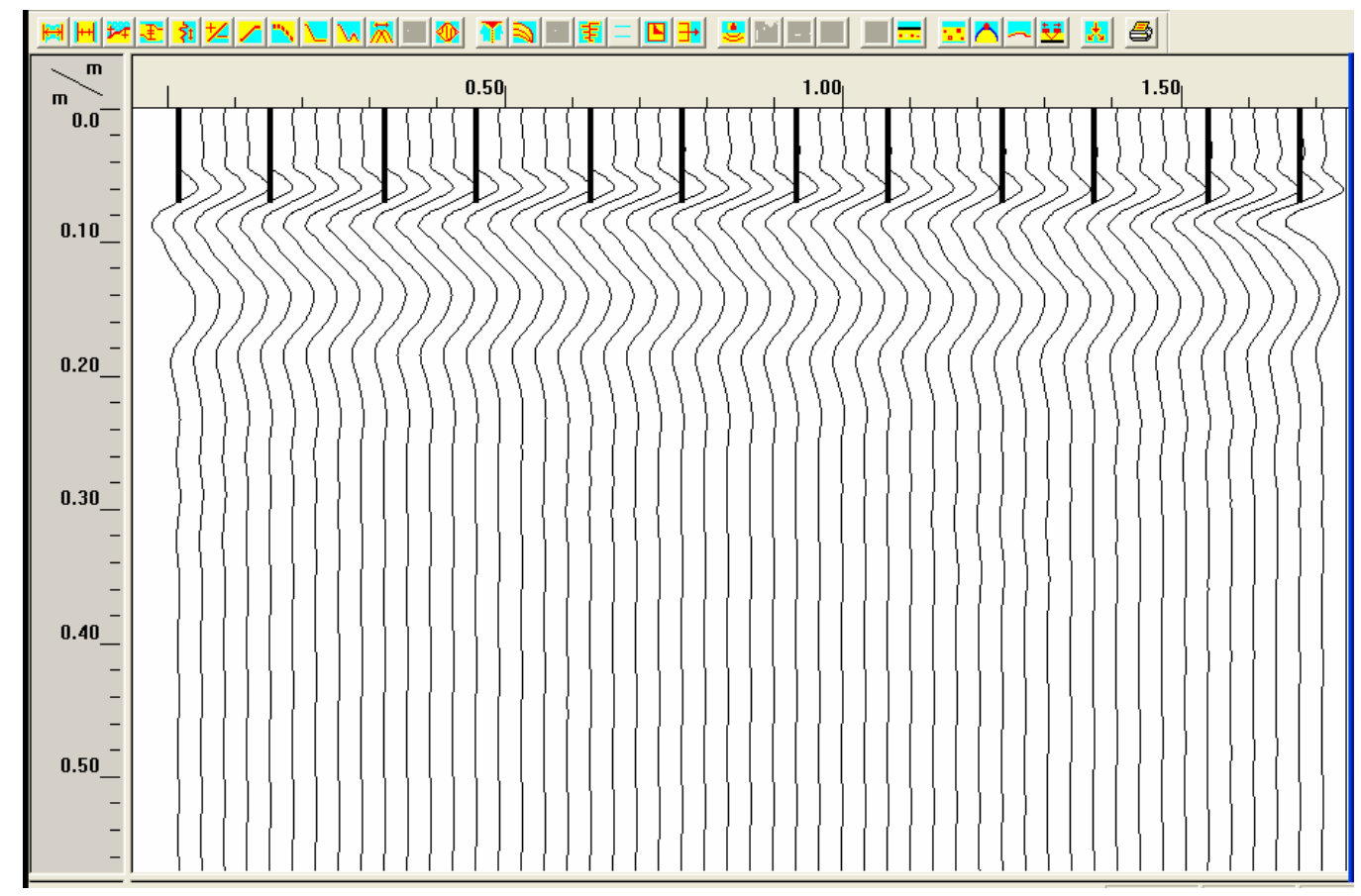

(a)

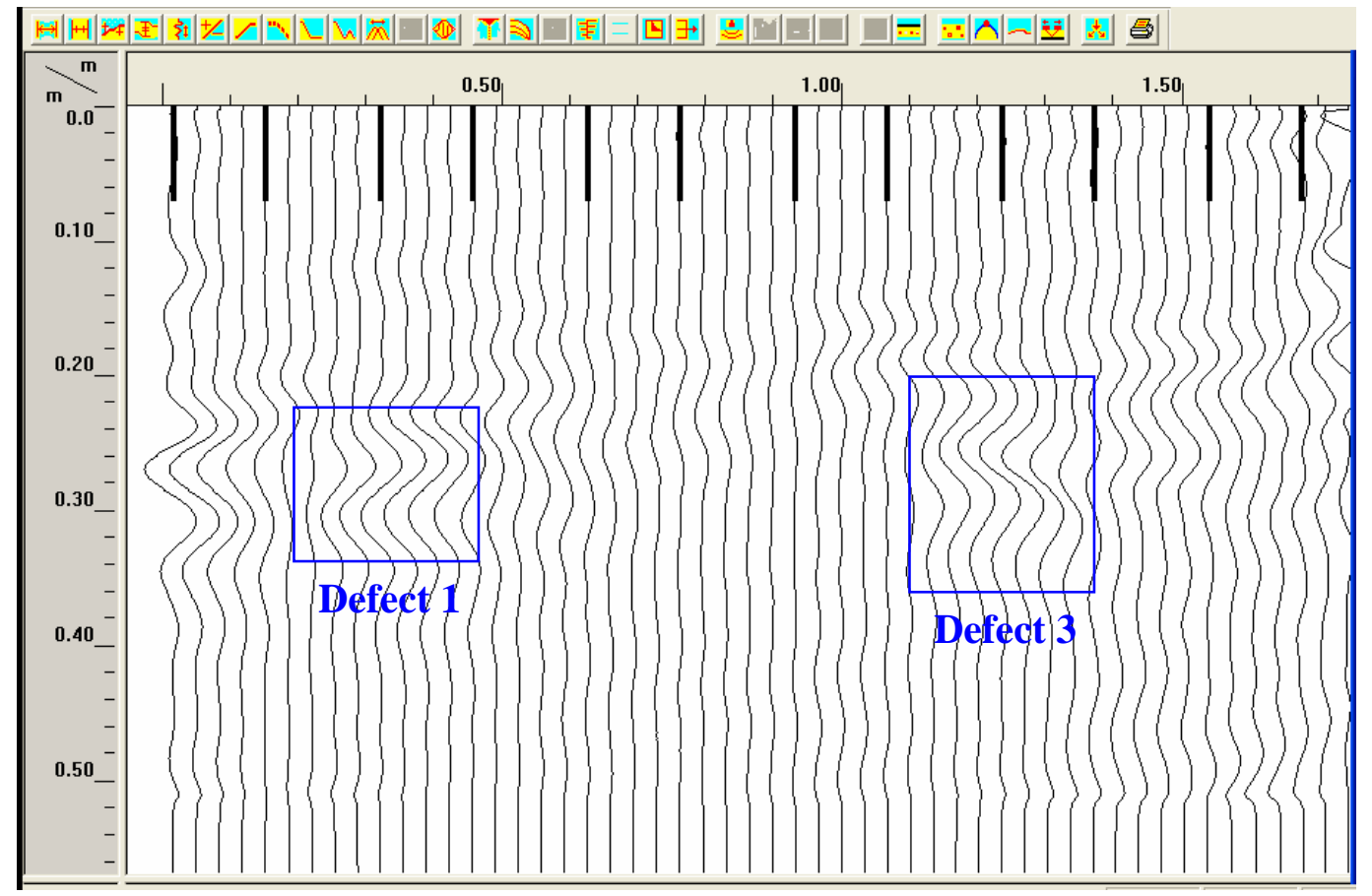

(b)

Figure 5-16 Wiggle plot of Log 2 along mark 5, horizontal scale indicating travel distance along the length of the $\log (\mathrm{m})$ and vertical scale indicating the depth $(\mathrm{m})$, for (a) raw data, and (b) processed data 


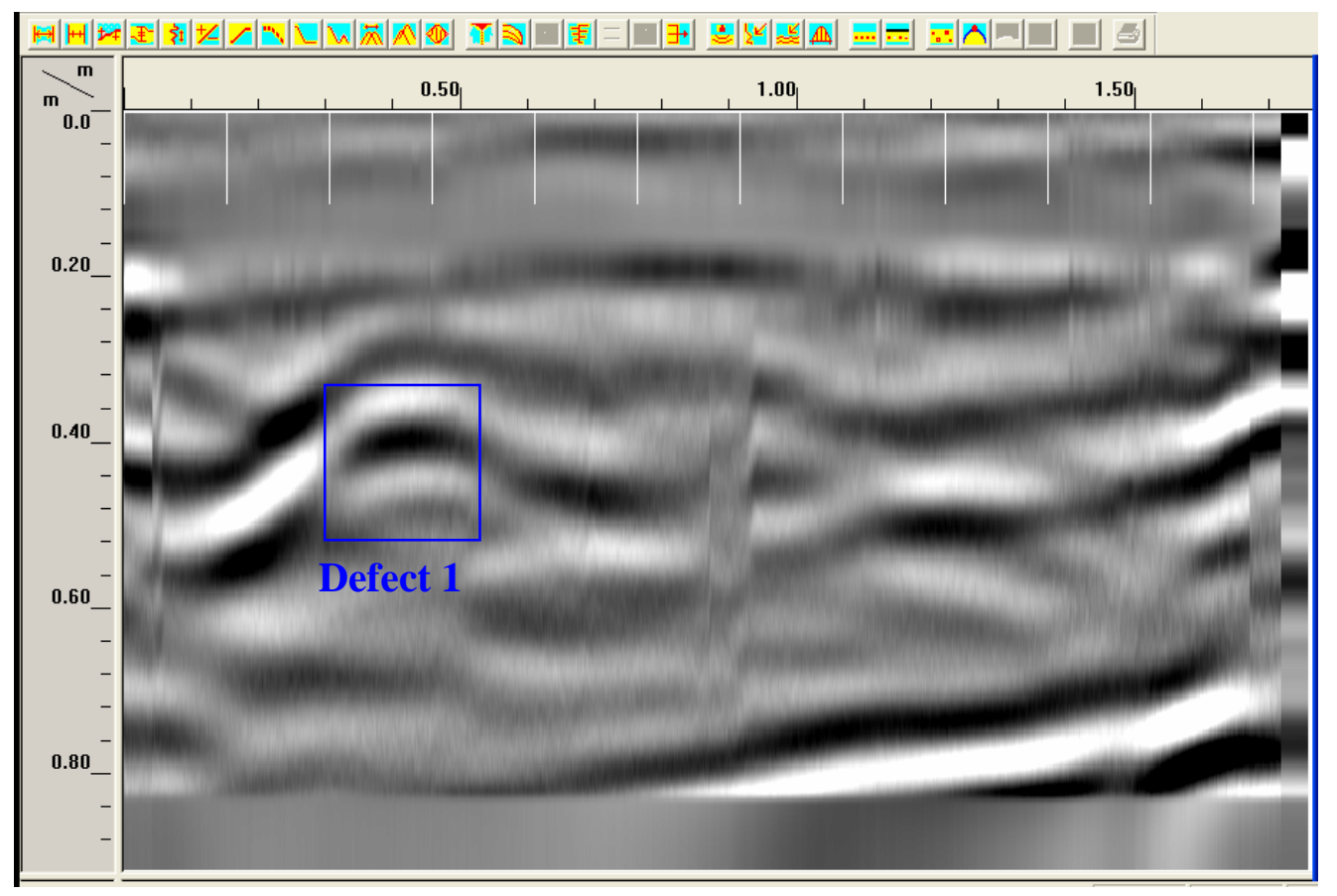

Figure 5-17 Processed GPR data of Log 2 along mark 2, horizontal scale indicating the travel distance along the length of the $\log (\mathrm{m})$ and the vertical scale indicating depth $(\mathrm{m})$

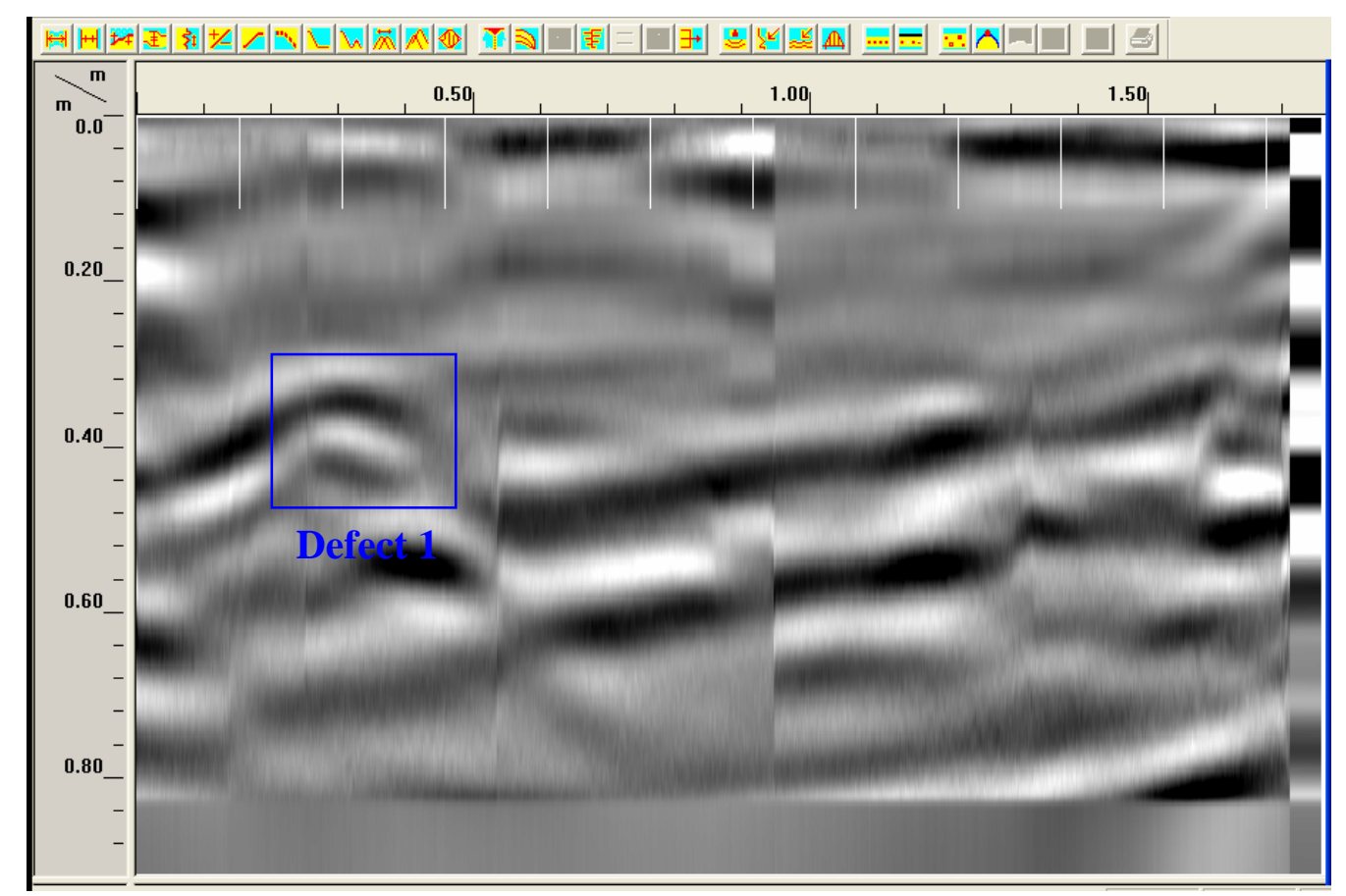

Figure 5-18 Processed GPR data of $\log 2$ along mark 8, horizontal scale indicating the travel distance along the length of the $\log (\mathrm{m})$ and the vertical scale indicating depth (m) 


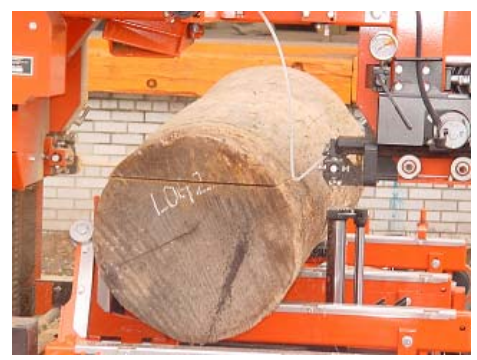

(a)

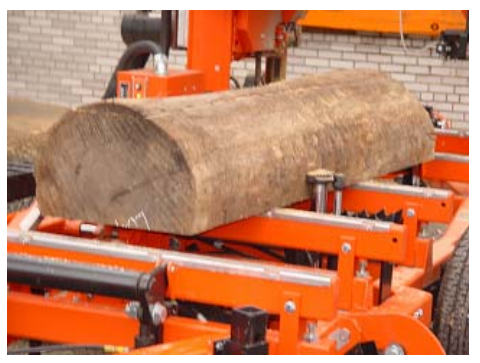

(b)

Figure 5-19 Sawing of Log 2 using a portable saw mill

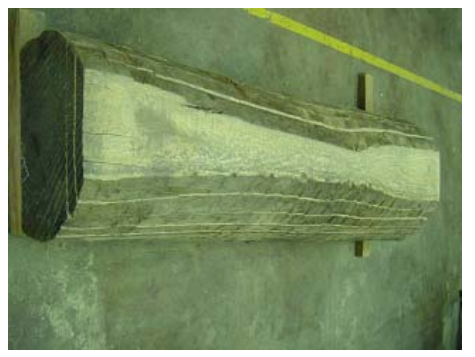

Figure 5-20 Horizontal slices of Log 2 after sawing

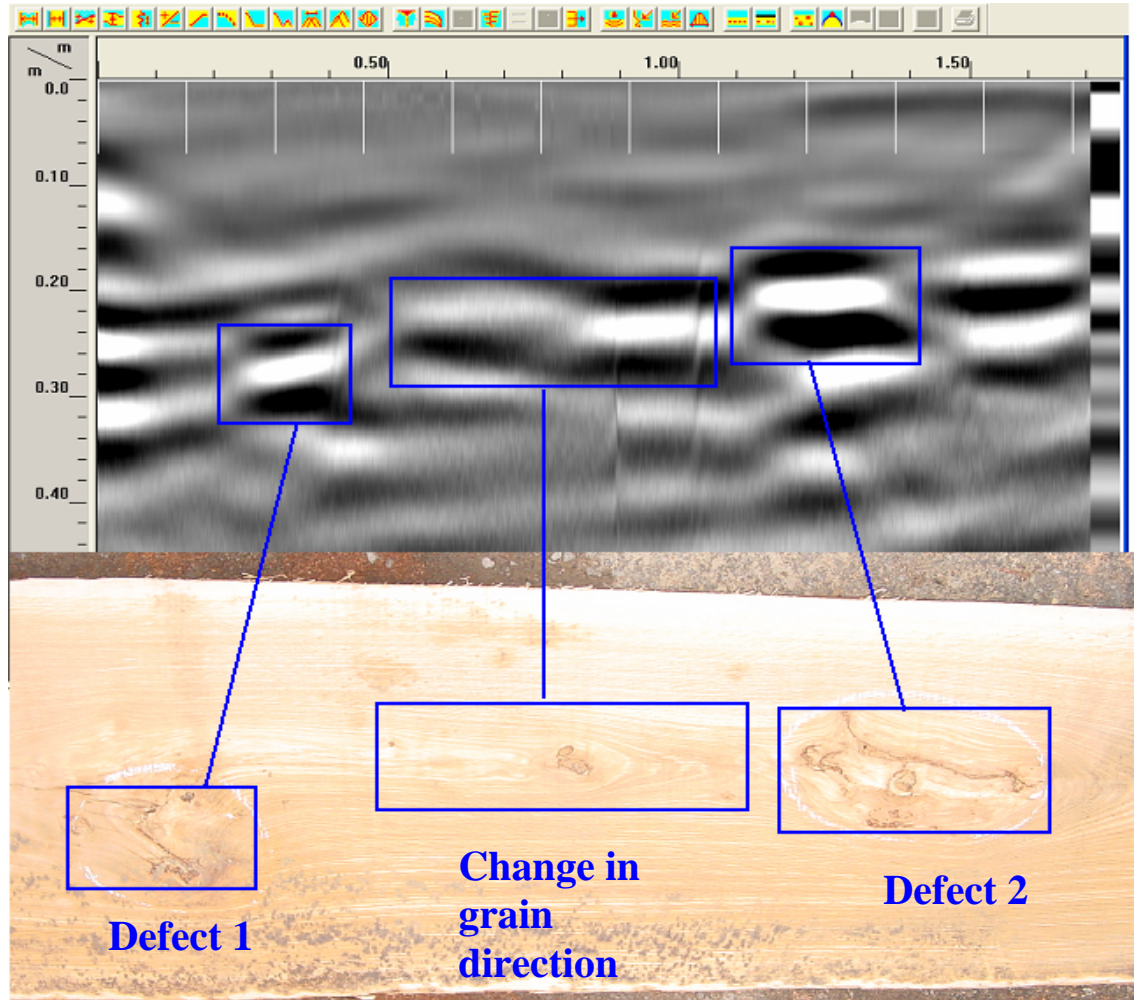

Figure 5-21 Processed data of Figure 5.12 and corresponding defects (knots) 


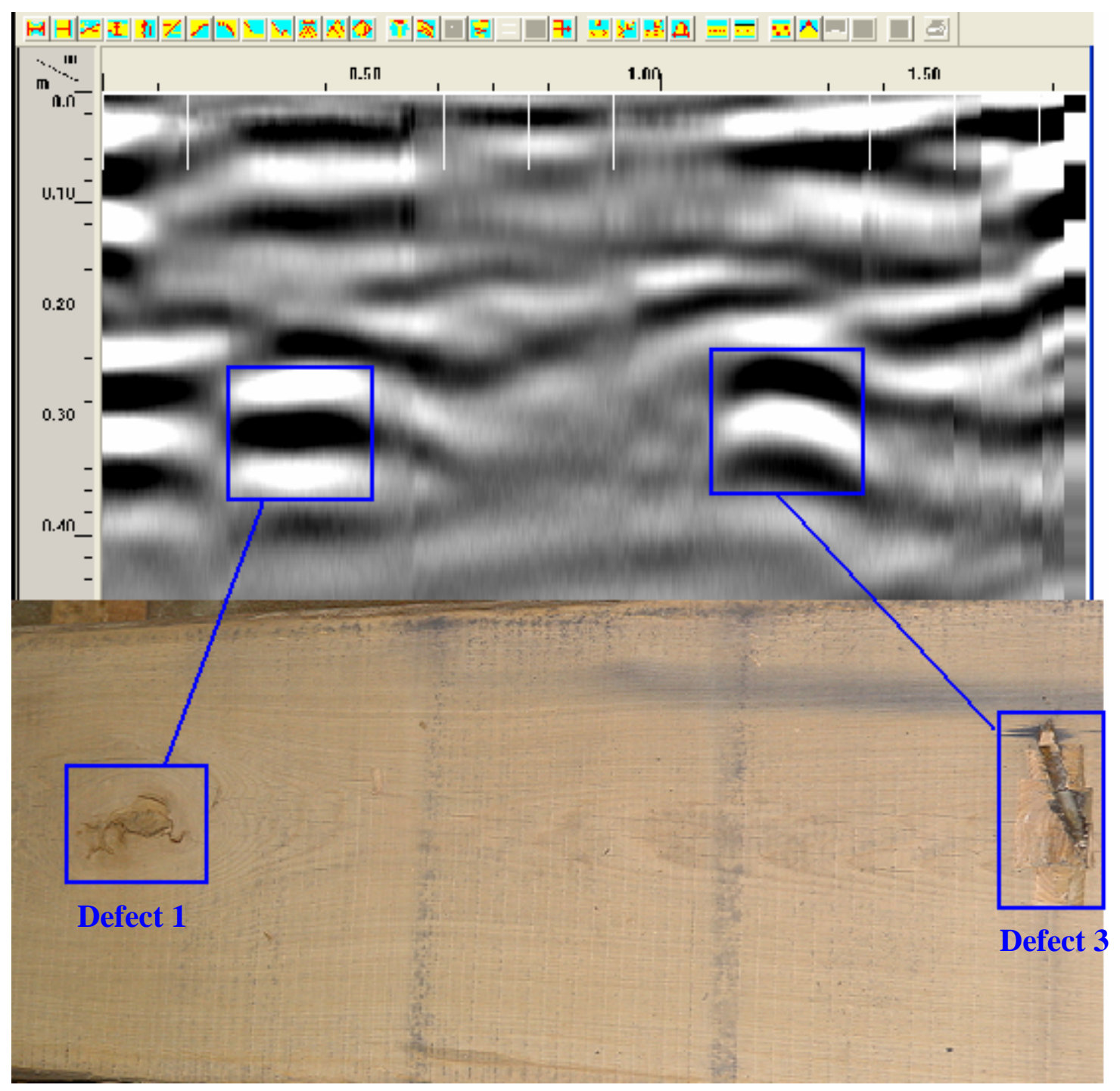

Figure 5-22 Processed data of Figure 5.15 and corresponding defects

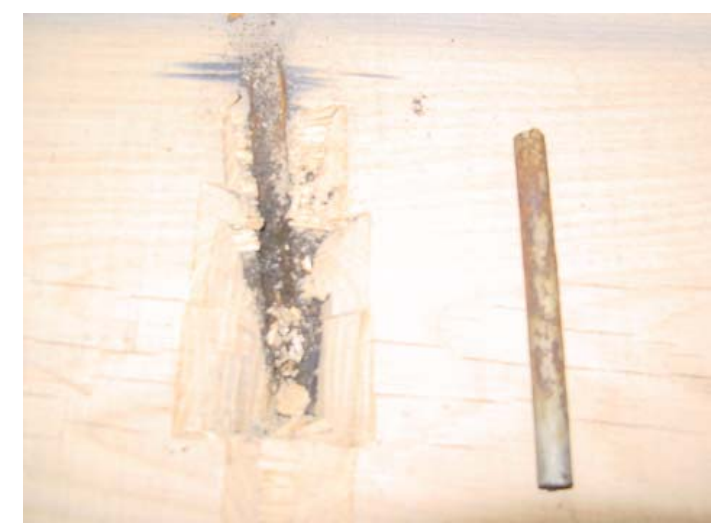

Figure 5-23 Inserted metal rod defect 


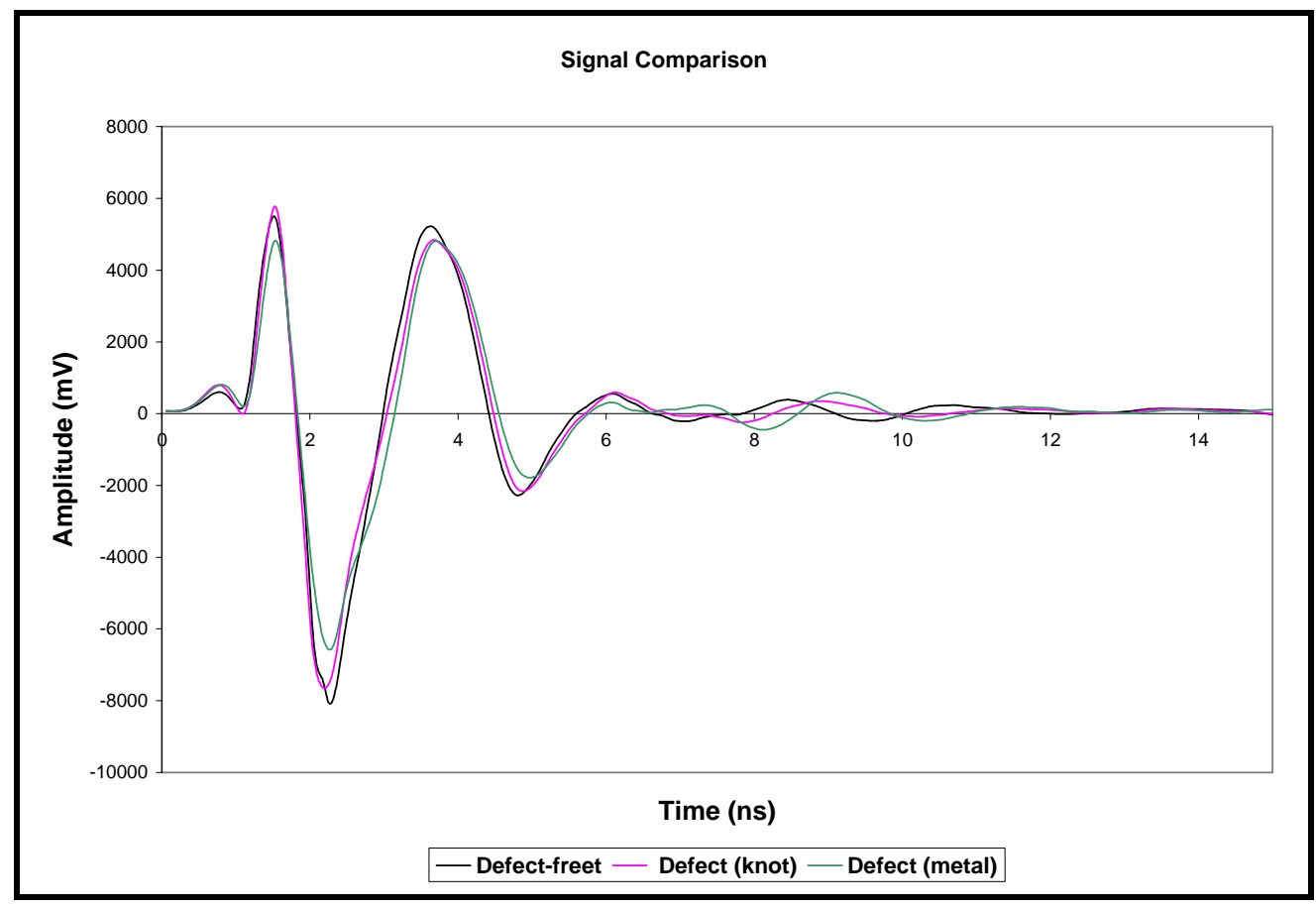

(a)

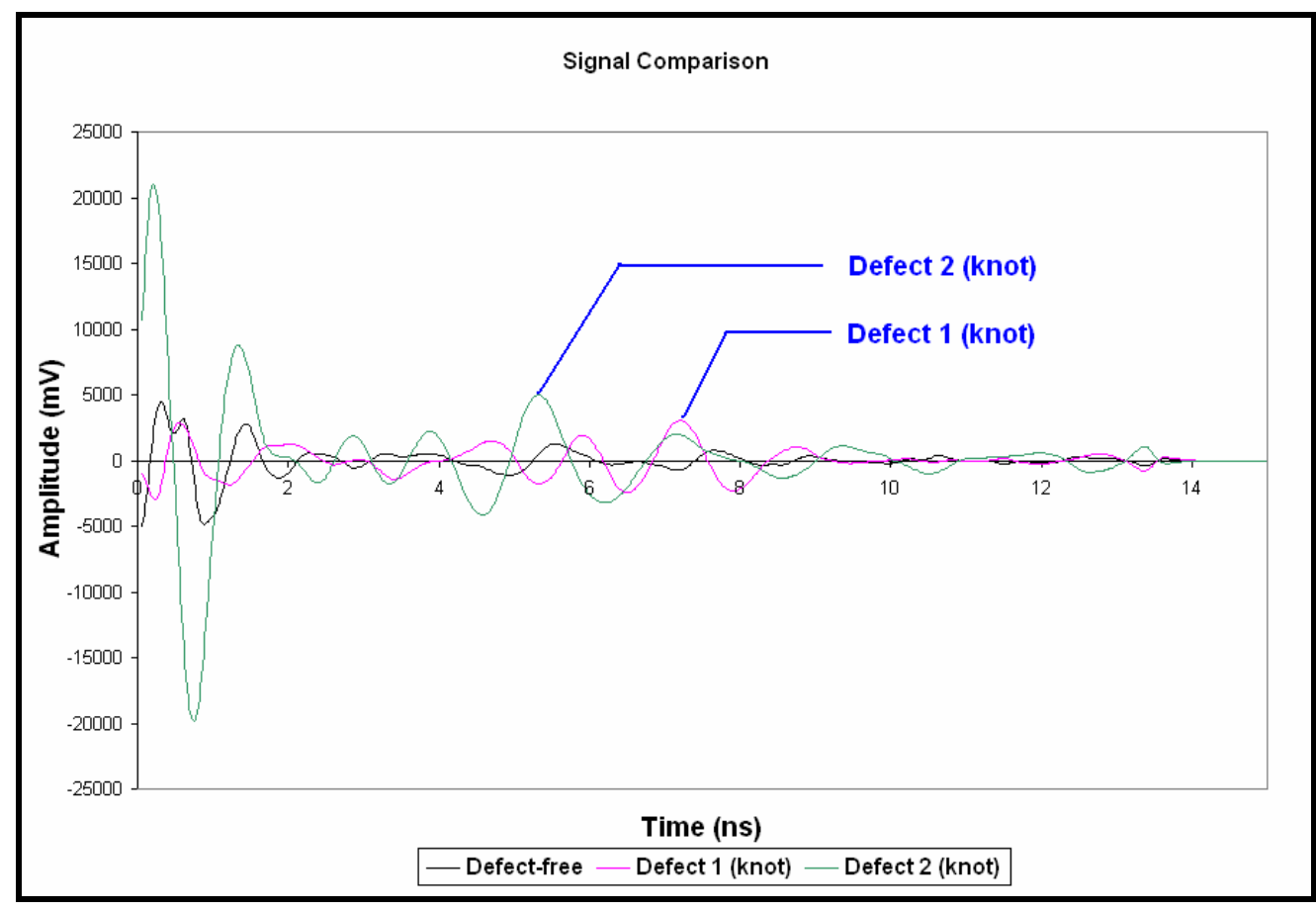

(b) 


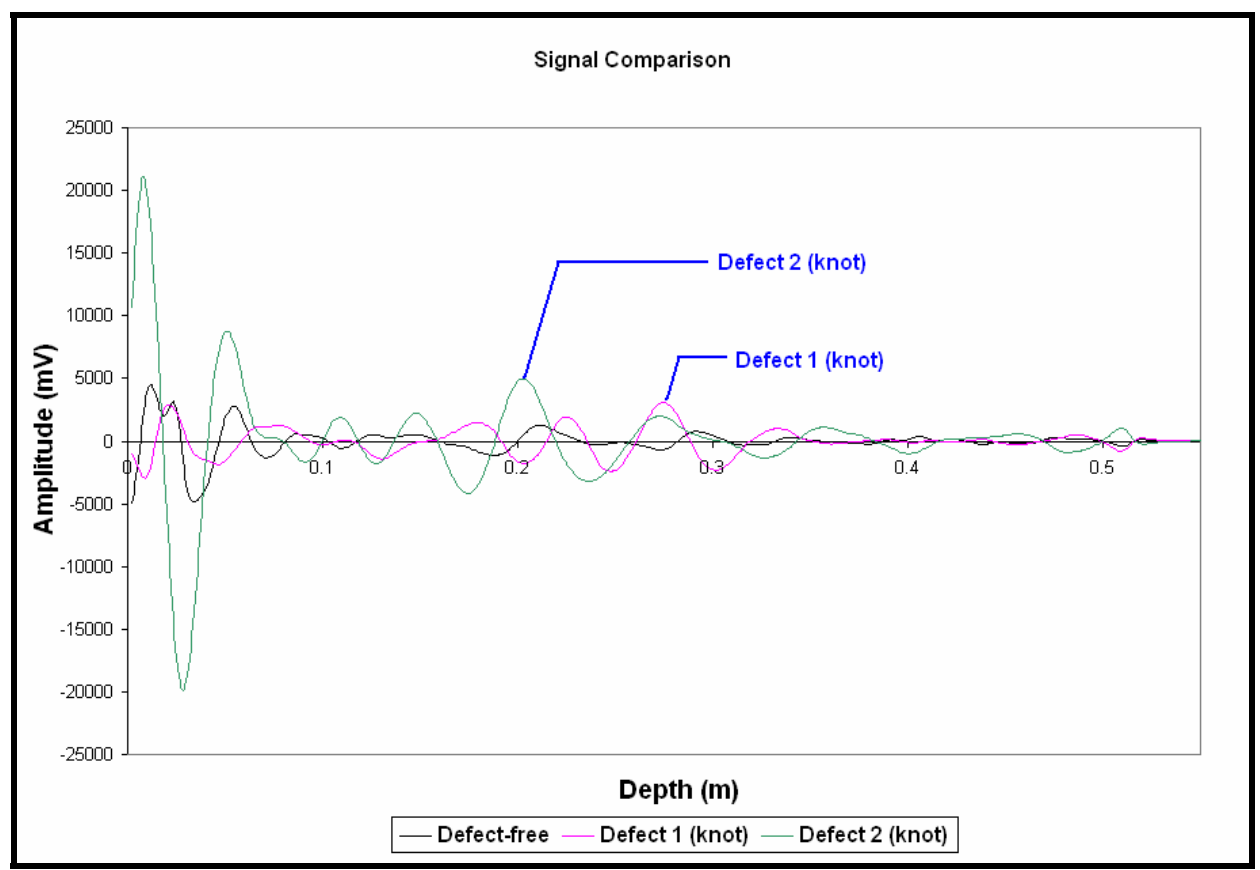

(c)

Figure 5-24 Comparison of GPR waveforms along mark 4 from sections with and without defects (knots) in Log 2 for (a) raw data (Amplitude vs. Time), (b) processed data (Amplitude vs. Time), and (c) processed data (Amplitude vs. Depth)

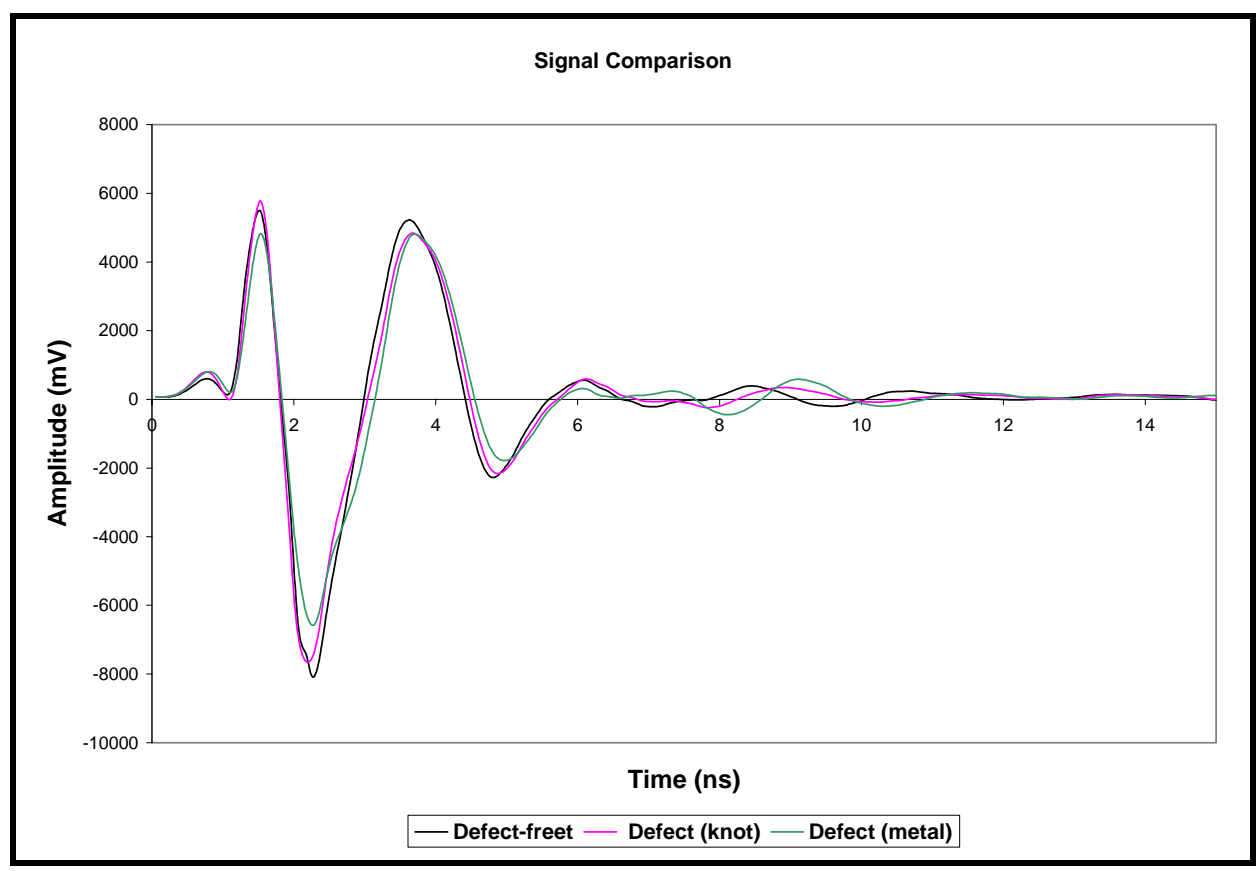

(a) 


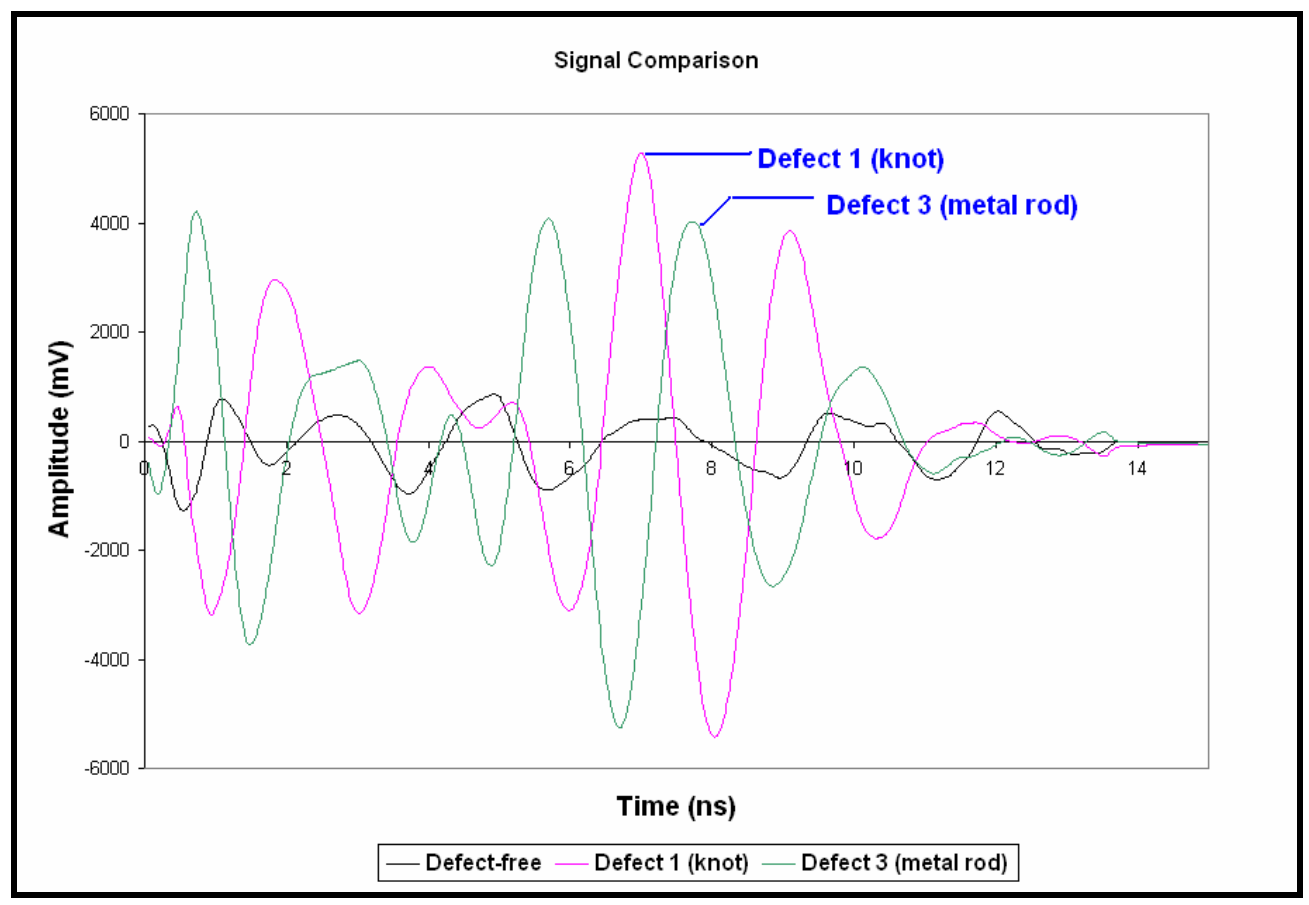

(b)

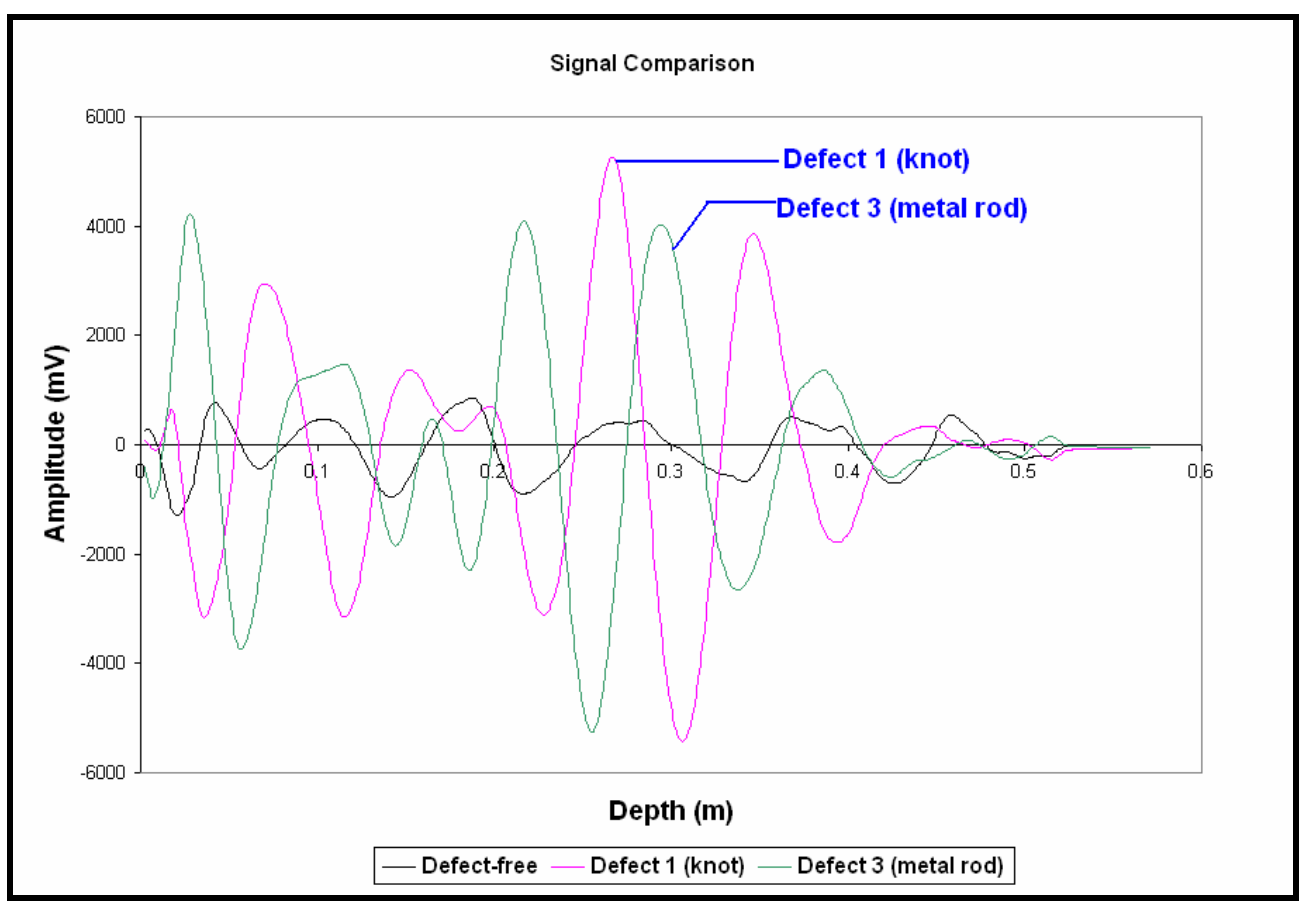

(c)

Figure 5-25 Comparison of GPR waveforms along mark 5 from sections with and without defects (knot and metal) in Log 2 for (a) raw data (Amplitude vs. Time), (b) processed data (Amplitude vs. Time), and (c) processed data (Amplitude vs. Depth) 


\section{$5.3 \quad$ LOG 3}

Table 5-3 Characteristics of $\log 3$

\begin{tabular}{|c|c|c|c|c|c|c|c|c|}
\hline $\begin{array}{c}\text { Log } \\
\text { no. }\end{array}$ & Species & $\begin{array}{c}\text { Length } \\
(\mathbf{m})\end{array}$ & $\begin{array}{c}\text { Diameter (m) } \\
\text { Butt } \\
\text { end }\end{array}$ & $\begin{array}{c}\text { Small } \\
\text { end }\end{array}$ & Surface & Max. & Average & $\begin{array}{c}\text { Dielectric } \\
\text { Constant }\end{array}$ \\
\hline 3 & Beech & 1.98 & 0.38 & 0.35 & 12 & 30 & 20 & 14 \\
\hline
\end{tabular}

The characteristics of $\log 3$ are given in Table 5.3. Figure 5-26 shows $\log 3$ and the markings made for scanning and sawing. The decay in the center of the log was very clearly visible. To check the extent of decay inside the log, a metal rod of $2 \mathrm{~m}$ length was inserted in the log as shown in Figure 5-27. The rod could easily be inserted from one end of the log to the other end, which indicated that the decay covered the entire length of the $\log$. The log was scanned using a single antenna, positioned on the top of the log, as shown in Figure 5-28. The data collection and processing parameters are shown in Figure 5-29. These parameters are explained in detail in Sections 4.1 and 4.2.

The process of analysis of this $\log$ was similar to that for $\log 1$. Figure 5-30 shows the raw GPR data for the scan with the antenna positioned over mark 1. The top set of white, black, and white bands indicates the transmit signal (antenna to log coupling). The second set of echo (black and white band) extending from the butt end to the small end was visible at 4.5 to $6 \mathrm{~ns}$ range. This was due to decay (rot) which extended all the way from the butt end to the small end. Also, from the raw GPR scan another internal characteristic, though weak, could be predicted at a distance of $0.75 \mathrm{~m}$ from the start of the scan. The bottom of the log could not be predicted from the raw data, which means the rot caused high signal attenuation.

The processed data is shown in Figure 5-31. From Figure 5-31(b), the extent of decay was predicted to be at a depth varying between $0.2 \mathrm{~m}$ and $0.13 \mathrm{~m}$. Also, another internal feature having a parabolic shape could be predicted at depth of $0.19 \mathrm{~m}$ and a distance of $0.2 \mathrm{~m}$ from the start of the scan. The defect at distance of $0.75 \mathrm{~m}$ became 
more prominent after processing the raw GPR scan. The processed GPR scan in Figure 531(b) also shows the bottom of the log very clearly.

Figure 5-32(a) and (b) show the wiggle plots of raw and the processed GPR scan, respectively. These waveforms were of very little use in understanding and locating the defects.

To validate the predication from the data, the log was sawn into horizontal layers parallel to mark 1 and the defects were located. The processed GPR scan (Figure 5-31(b)) and the corresponding defects are shown in Figure 5-33. After sawing, the log was found to be rotten in the center over its entire length from the butt end to the small end, which was also evident from the GPR scan as mentioned earlier. The extent of decay (rot) can be seen in Figures 5-34. The parabolic internal feature at $0.19 \mathrm{~m}$ depth and $0.2 \mathrm{~m}$ distance in the GPR scan (Figure 5-33 - top picture) was due to the presence of metal nails. After sawing, the actual metal defects were located at a depth of $0.18 \mathrm{~m}$ and at distance of 0.2 $\mathrm{m}$ from the butt end of the log. There were several metal nails within a horizontal distance of $0.05 \mathrm{~m}$ (2"), whose echoes seemed to have merged into one echo in the GPR scan. These metal nails had diameter of $4 \mathrm{~mm}$ and length of around $0.06 \mathrm{~m}$. Figures 5-35 and 5-36 show the location of embedded metal nails and three metal nails pulled out of the log. The signal clutter near the $0.75 \mathrm{~m}$ distance at about $0.13 \mathrm{~m}$ depth in the GPR scan as shown in Figure 5-33 represents area of heavy rotting.

Figures 5-37(a) and 5-37(b) show individual waveforms extracted from raw and processed GPR scans from two defective sections for comparison. Since the entire core of the log was rotten, no defect-free signal was available for this log. These figures show the waveform amplitudes on the vertical axis and the two-way travel time through the depth of the $\log$ on the horizontal axis. The positive peaks between 4 and 6 ns range in Figure 5-37(b) and 0.15 to $0.22 \mathrm{~m}$ range in Figure 5-37(c) indicate the presence of defects. From Figures 5-37(b) and 5-37(c) it can be seen that the metal defect is located at $5 \mathrm{~ns}(0.19 \mathrm{~m})$ range and the rot is located near $4 \mathrm{~ns}(0.17 \mathrm{~m})$ range. Also, the combined reflection from metal and rot is higher in amplitude than the reflection from the rot alone.

There was an excellent correlation between the predicated defects and their location in the GPR scan with the observations made after sawing the log. 


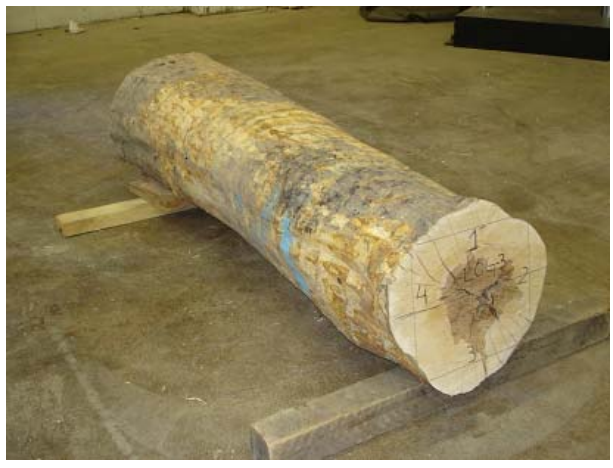

(a)

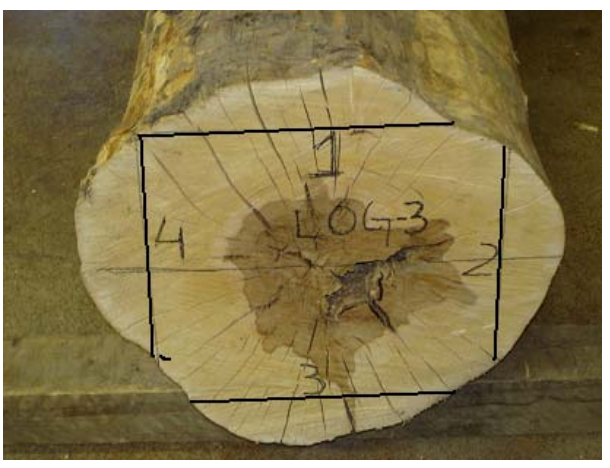

(b)

Figure 5-26 Pictures showing (a) Log 3 (b) the markings and the decay

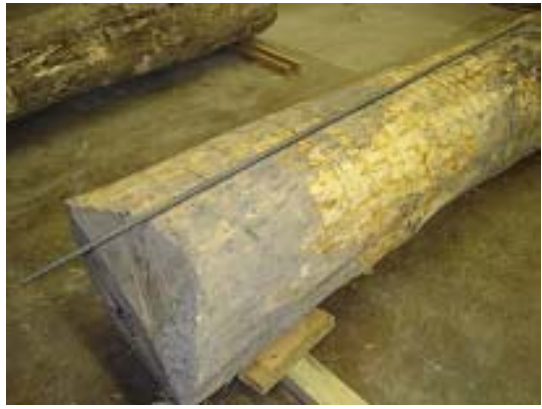

(a)

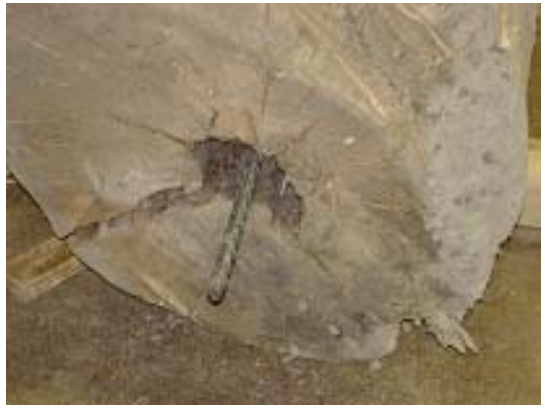

(b)

Figure 5-27 : Pictures showing (a) metal rod (b) the extent to which the metal could penetrate

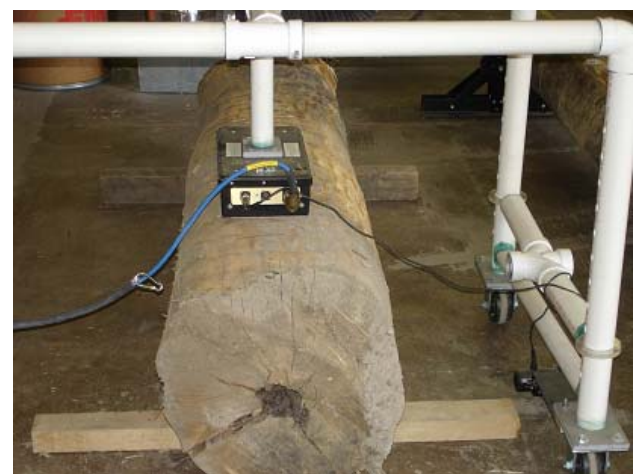

Figure 5-28 Antenna configuration 


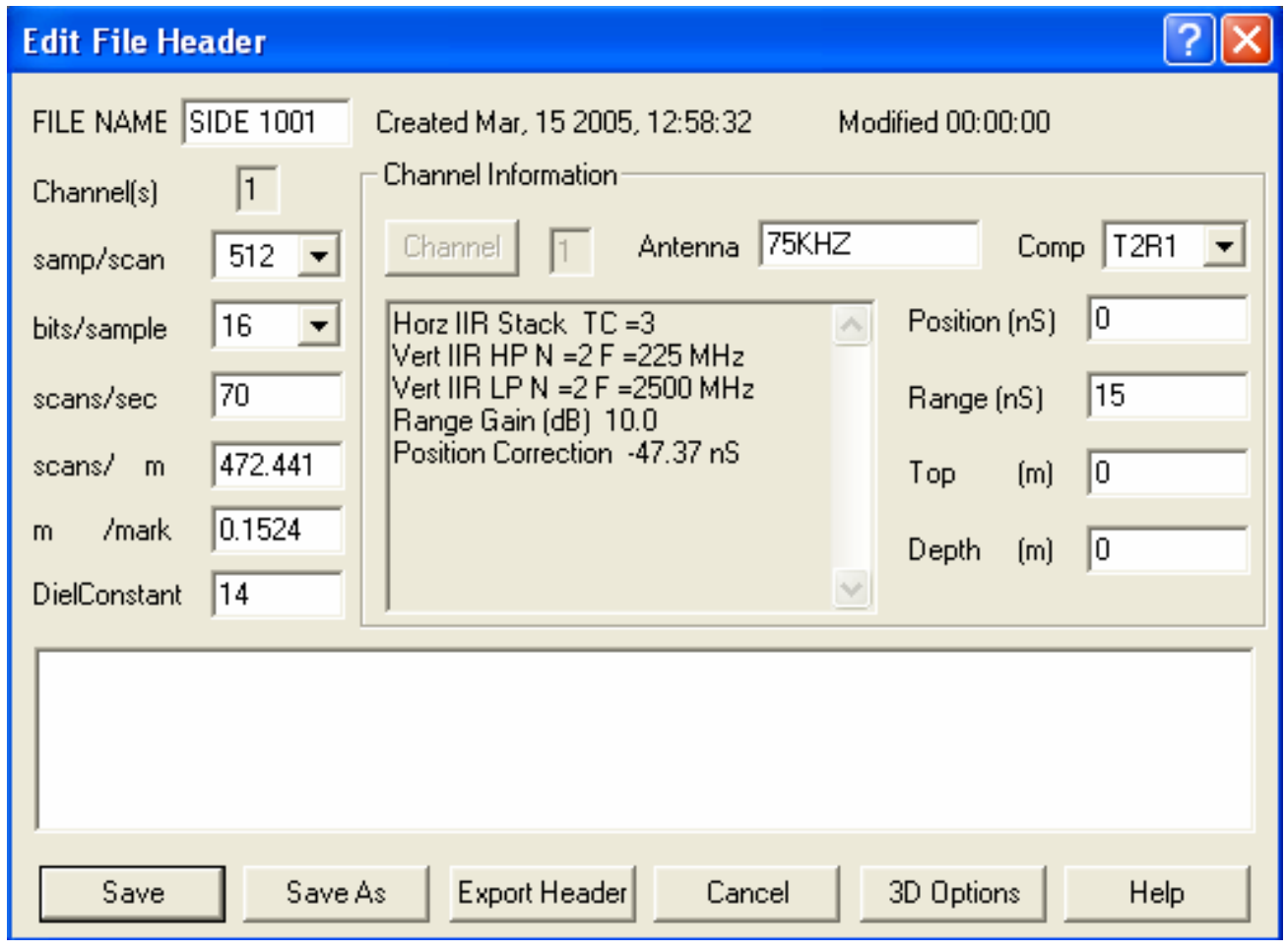

(a)

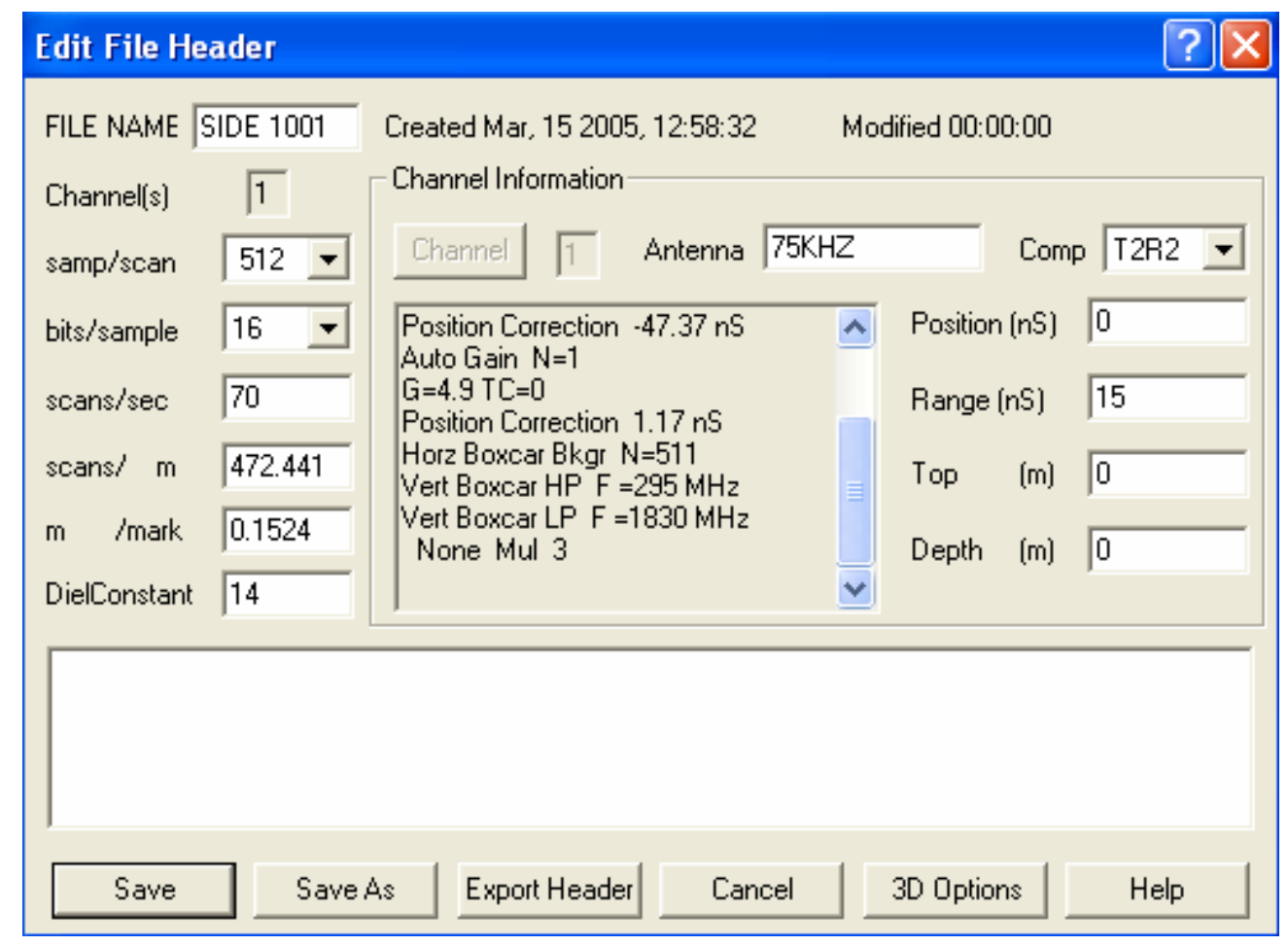

(b)

Figure 5-29 (a) Data collection parameters (b) data processing parameters 


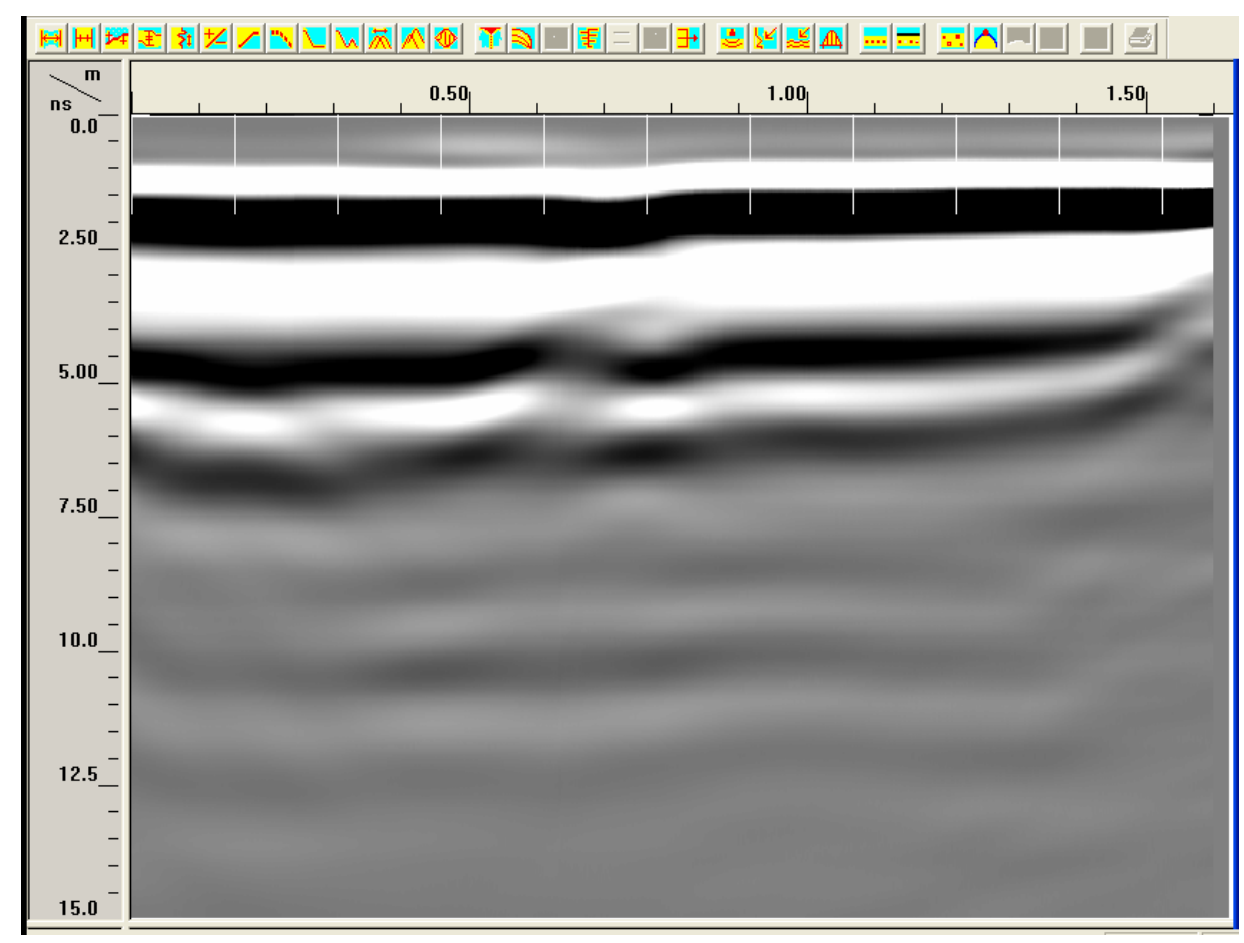

(a)

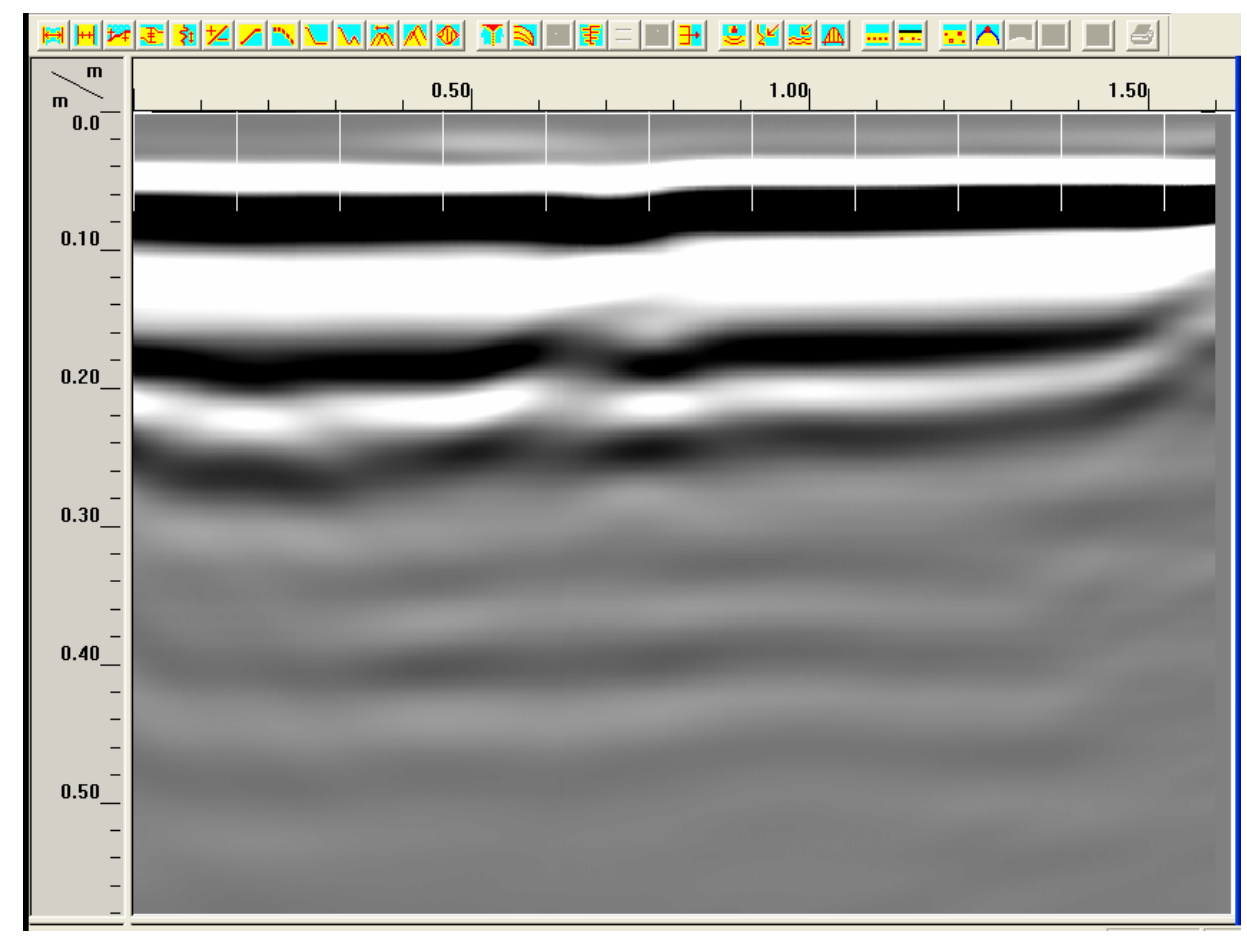

(b)

Figure 5-30 Raw GPR data of $\log$ 3, horizontal scale indicating the travel distance along the length of the $\log (\mathrm{m})$ and the vertical scale indicating (a) time (ns) (b) depth (m) 


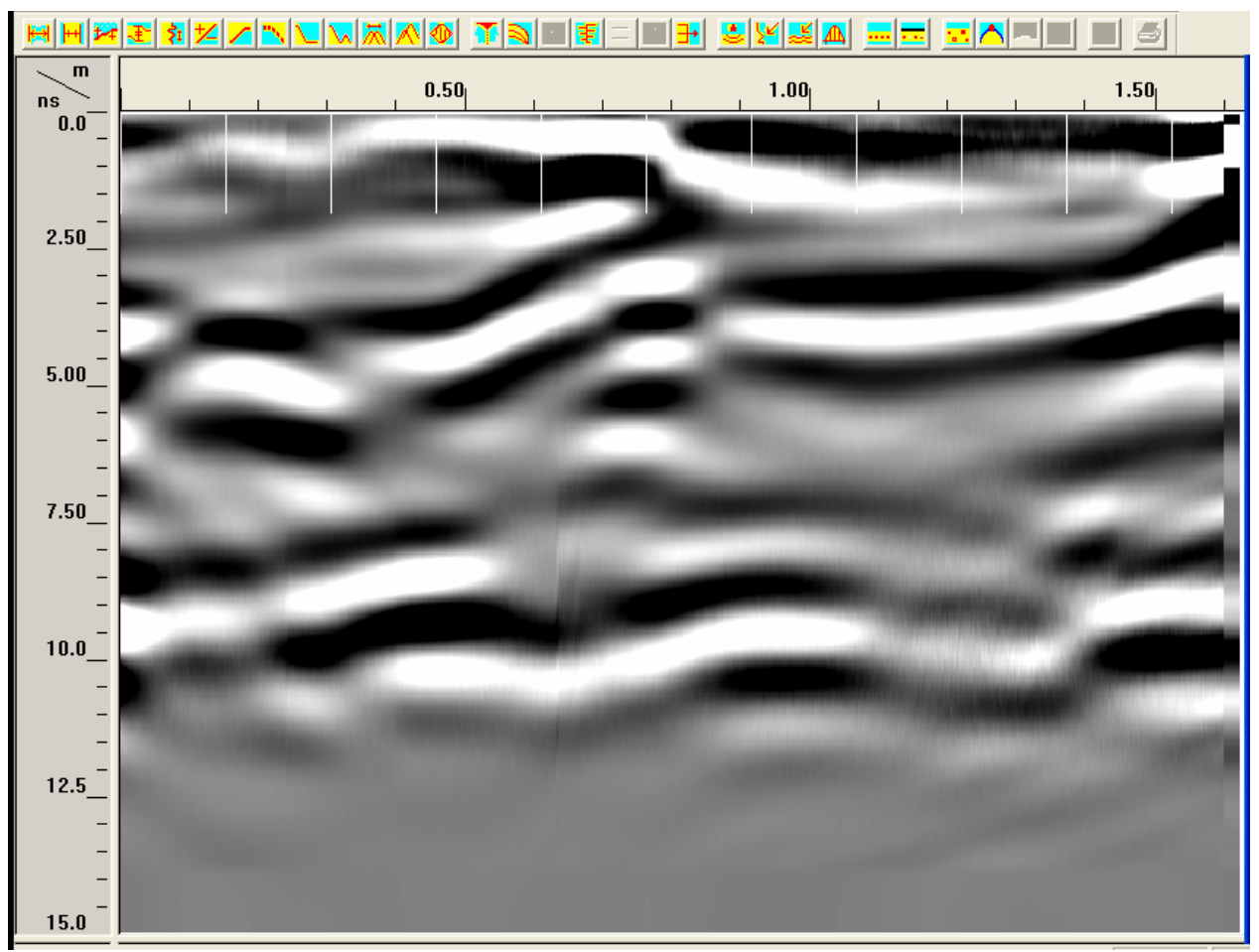

(a)

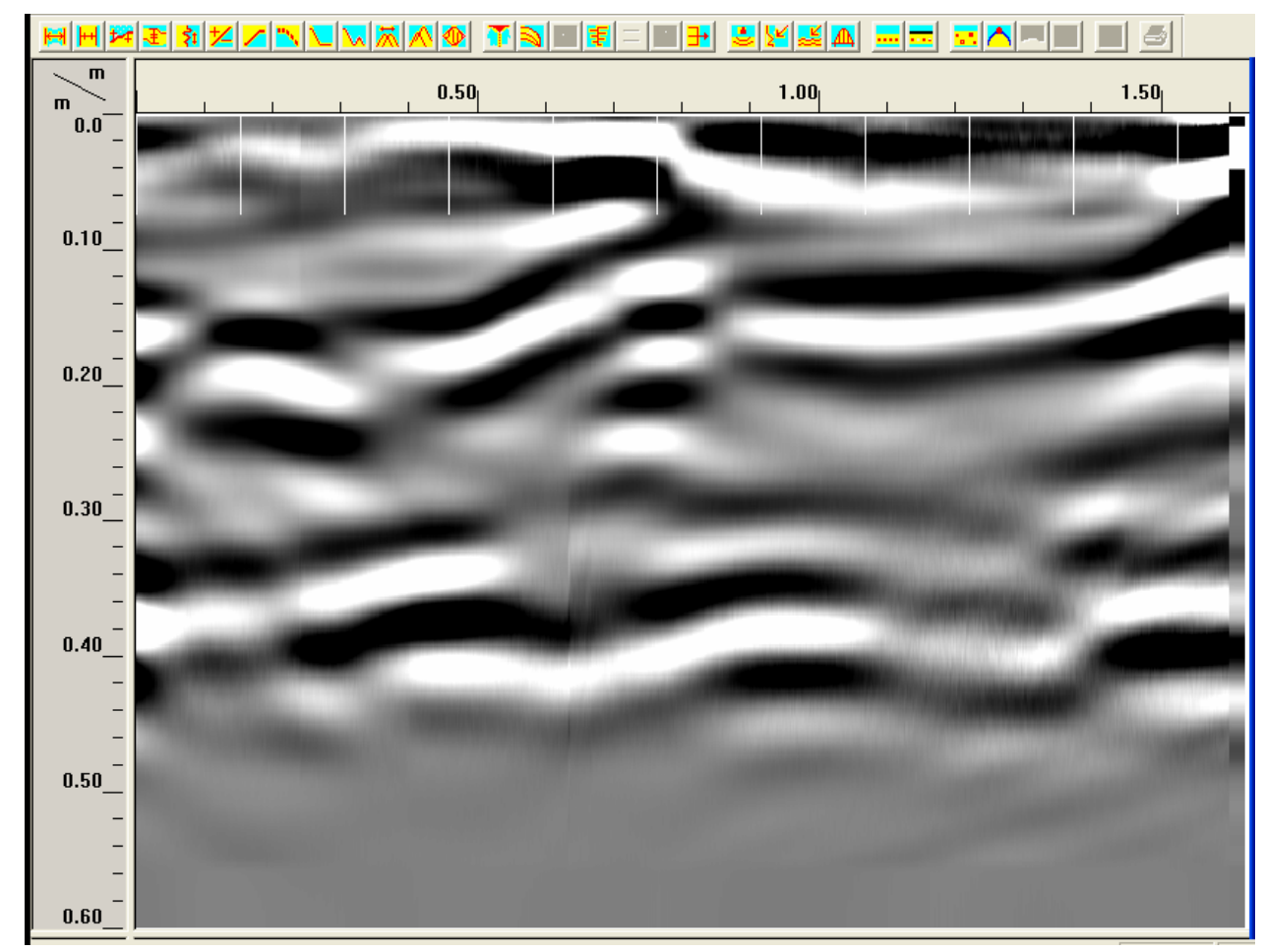

(b)

Figure 5-31 Processed GPR data of $\log 3$, horizontal scale indicating the travel distance along the length of the $\log (\mathrm{m})$ and the vertical scale indicating (a) time (ns) (b) depth (m) 


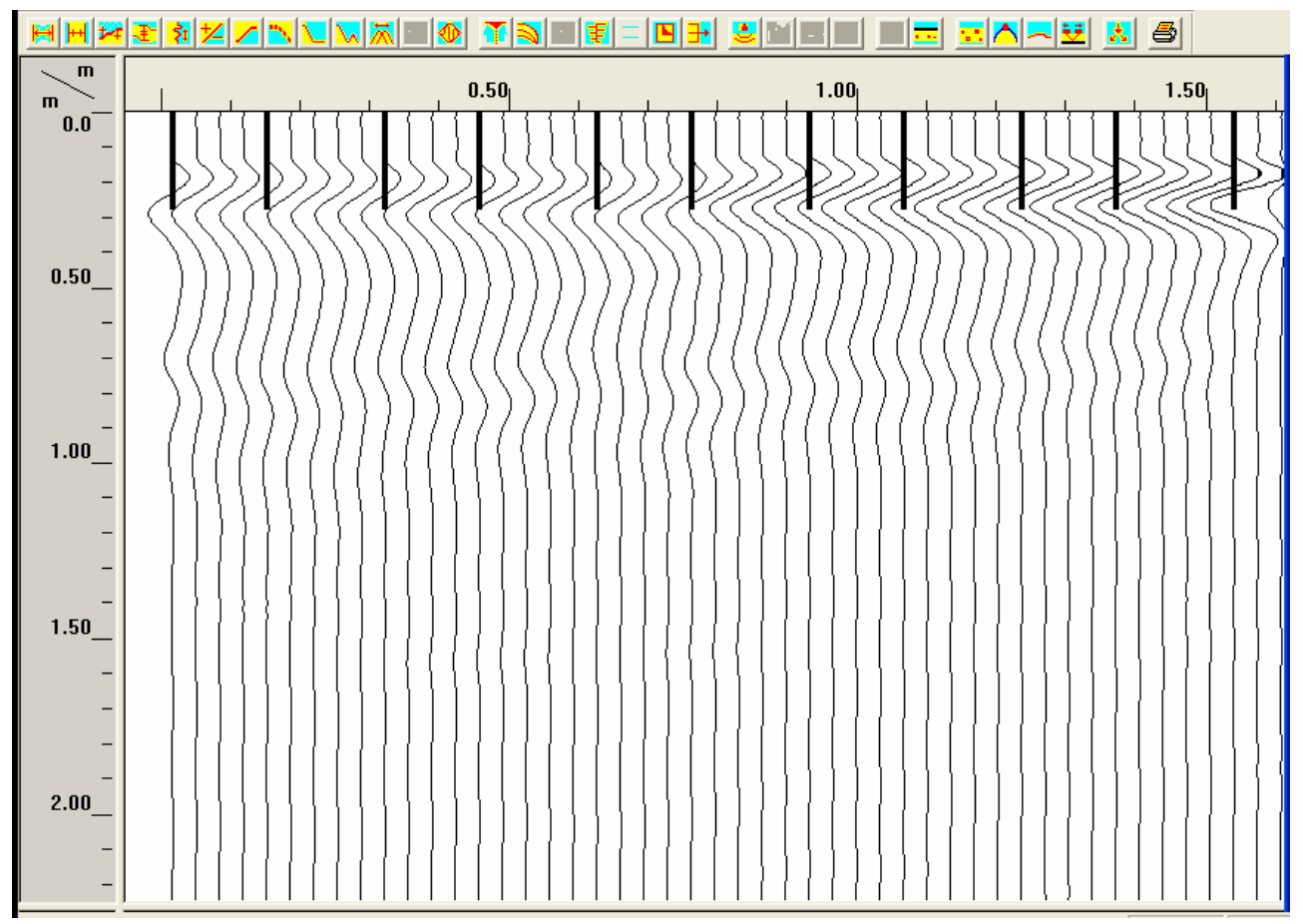

(a)

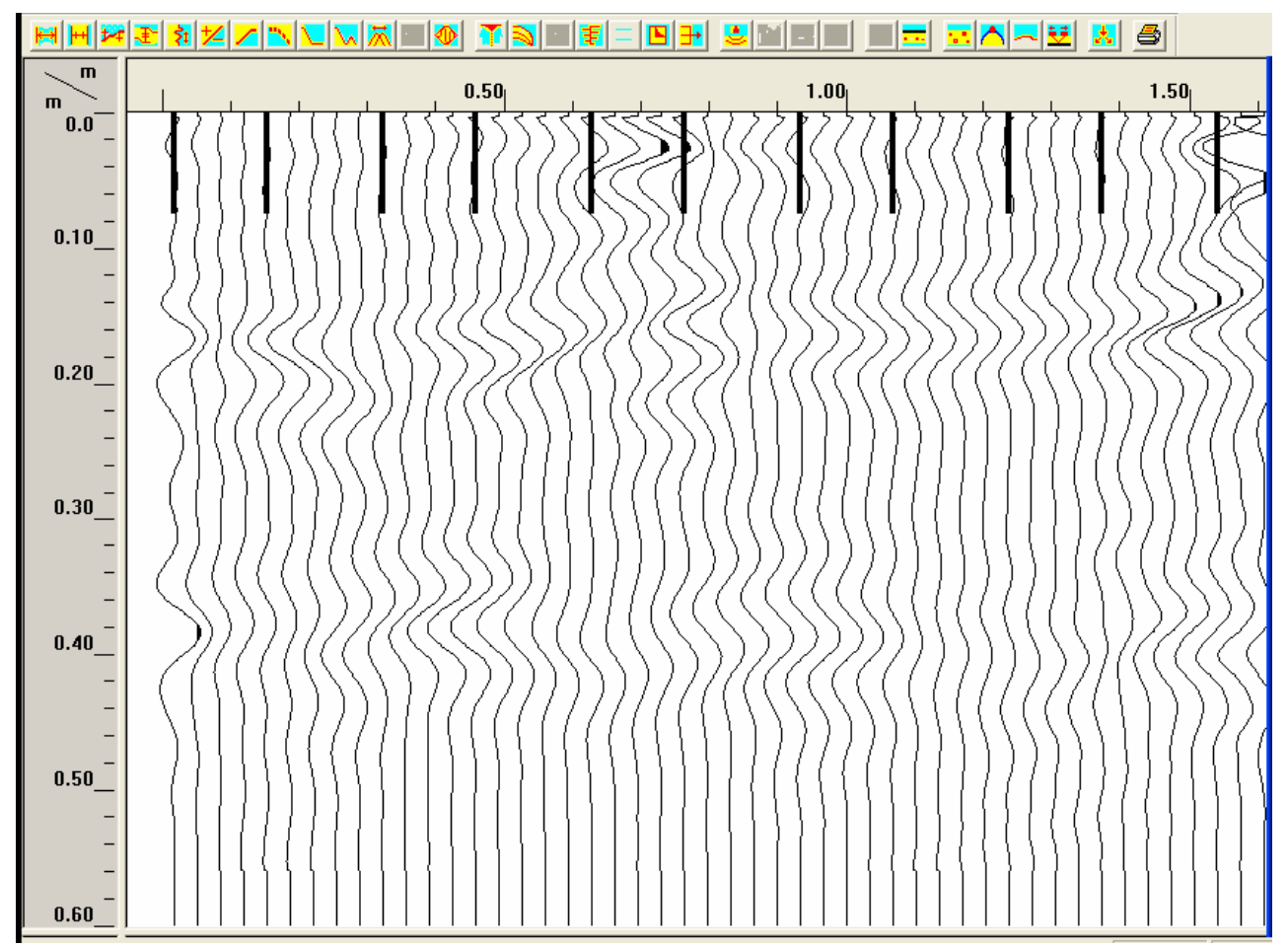

(b)

Figure 5-32 Wiggle plot of $\log 3$, horizontal scale indicating travel distance along the length of the $\log (\mathrm{m})$ and vertical scale indicating the depth $(\mathrm{m})$, for (a) raw data, and (b) processed data 


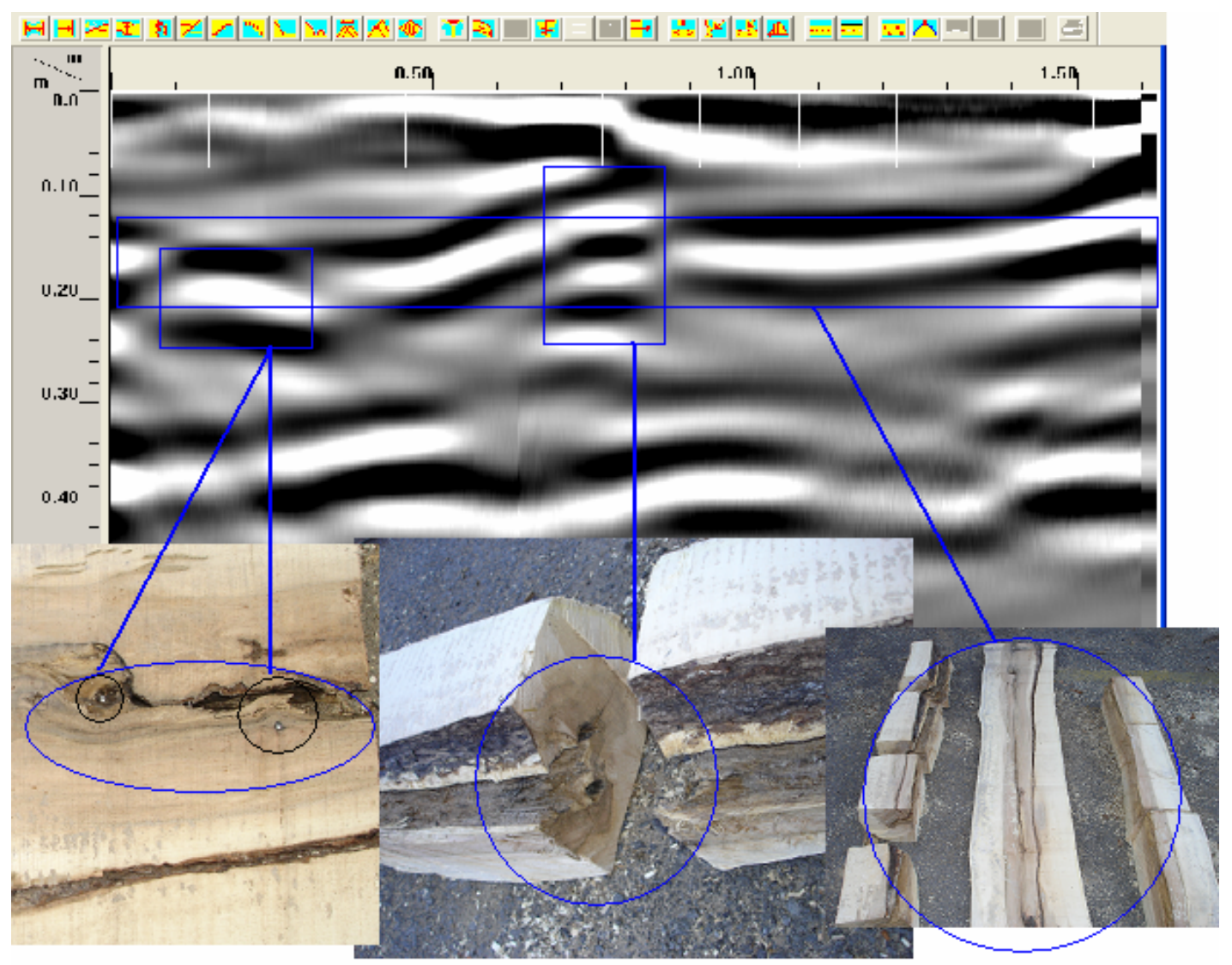

Figure 5-33 Processed data and corresponding defects in Log 3

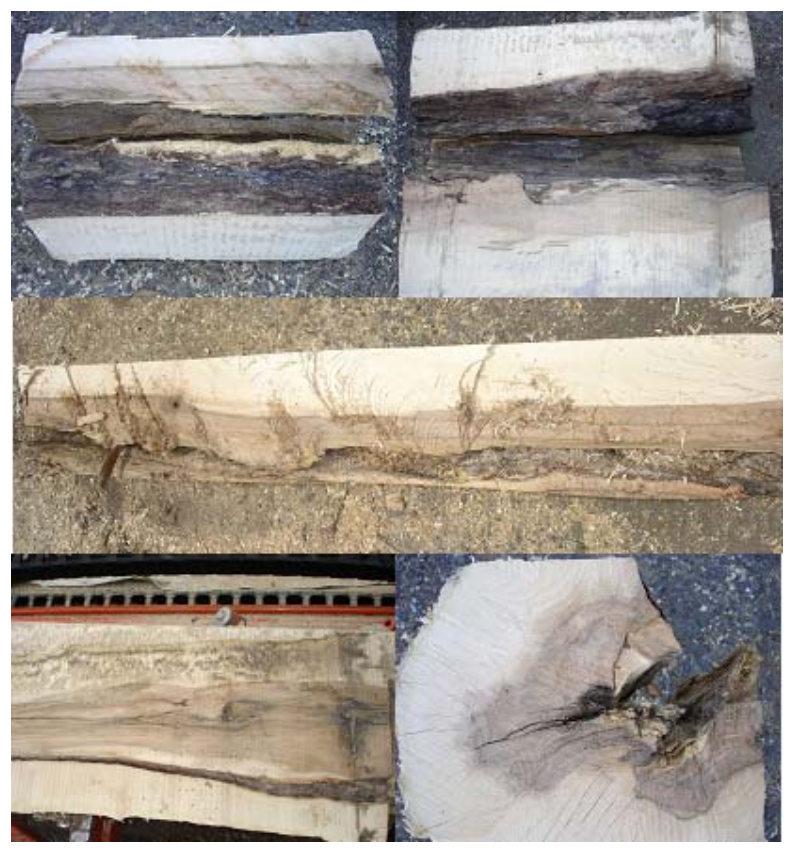

Figure 5-34 Pictures showing the extent of decay (rot) in Log 3 


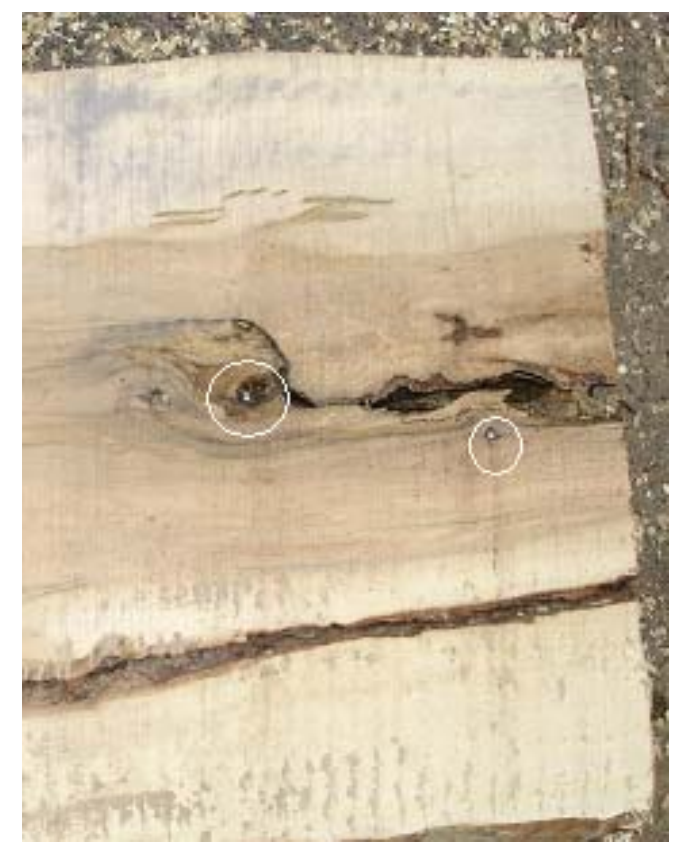

Figure 5-35 Location of metal nails

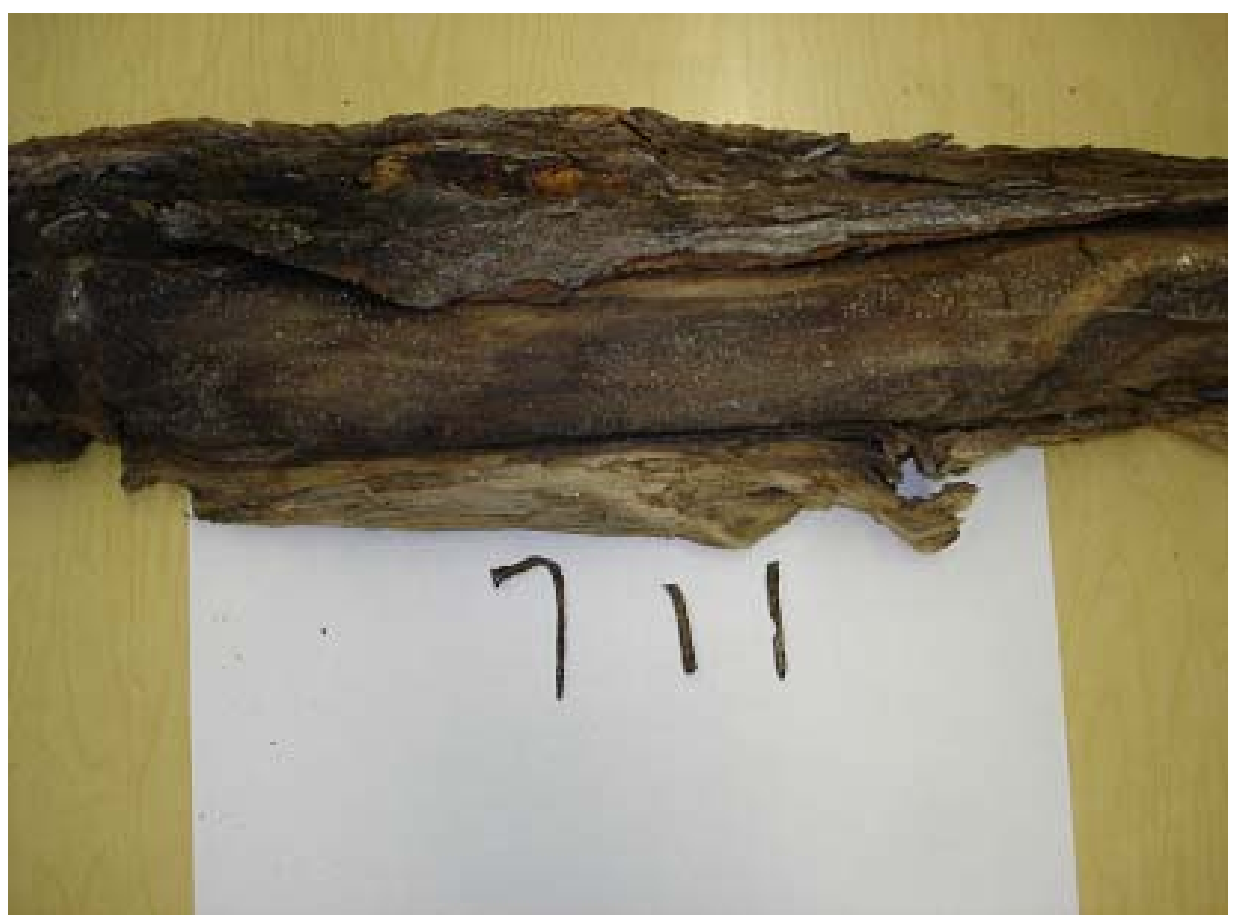

Figure 5-36 Rotten piece and metal nails extracted out from Log 3 


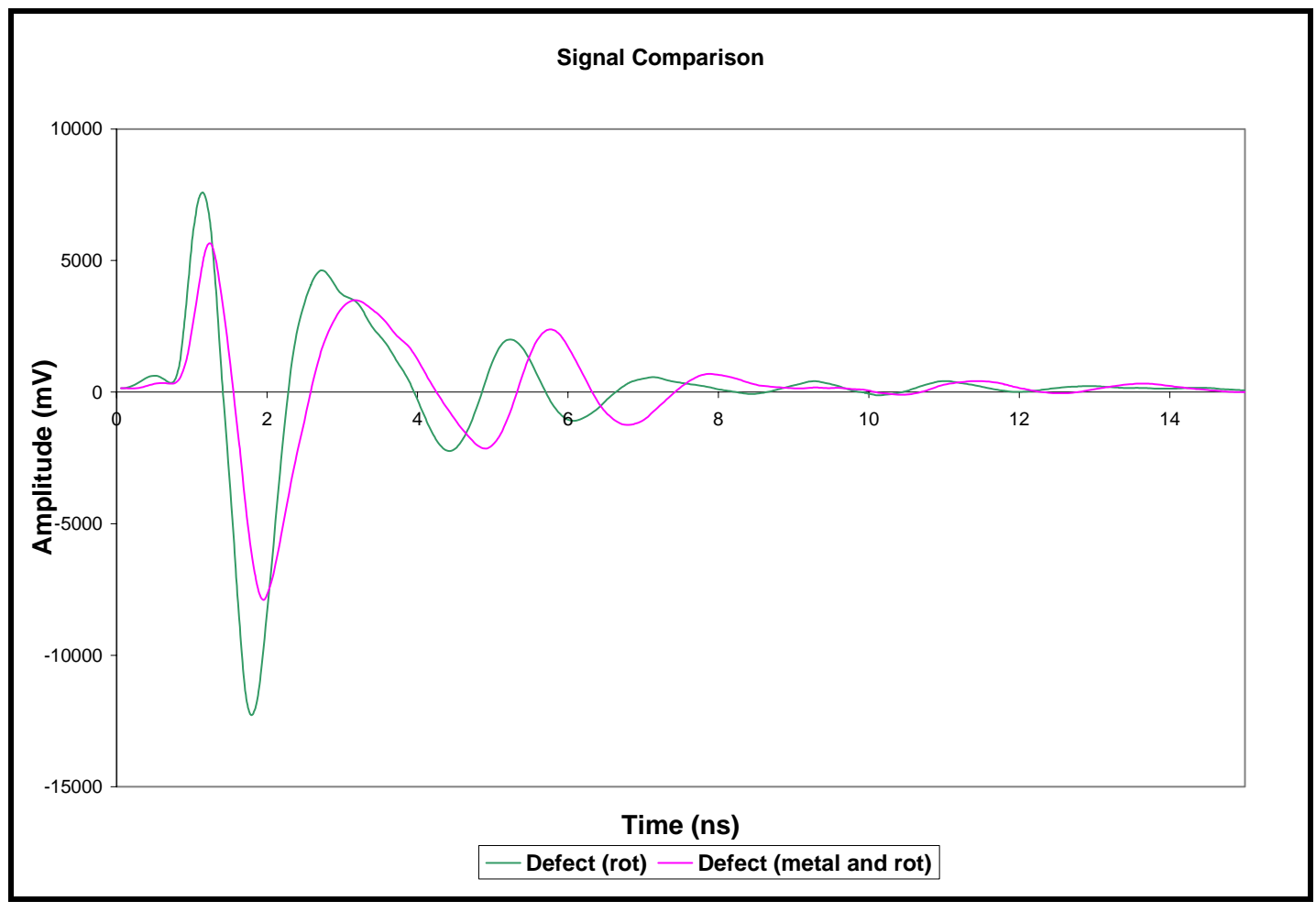

(a)

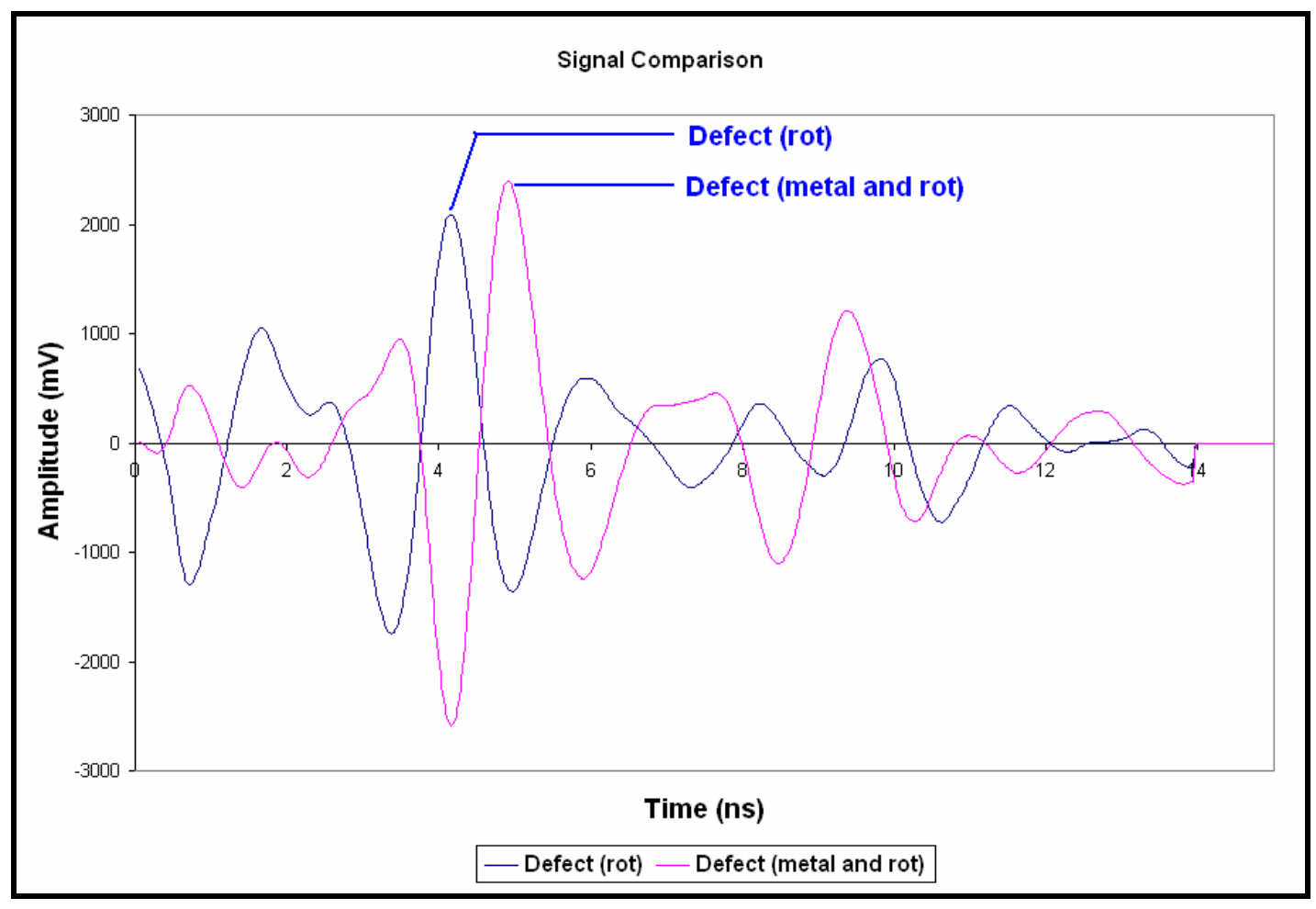

(b) 


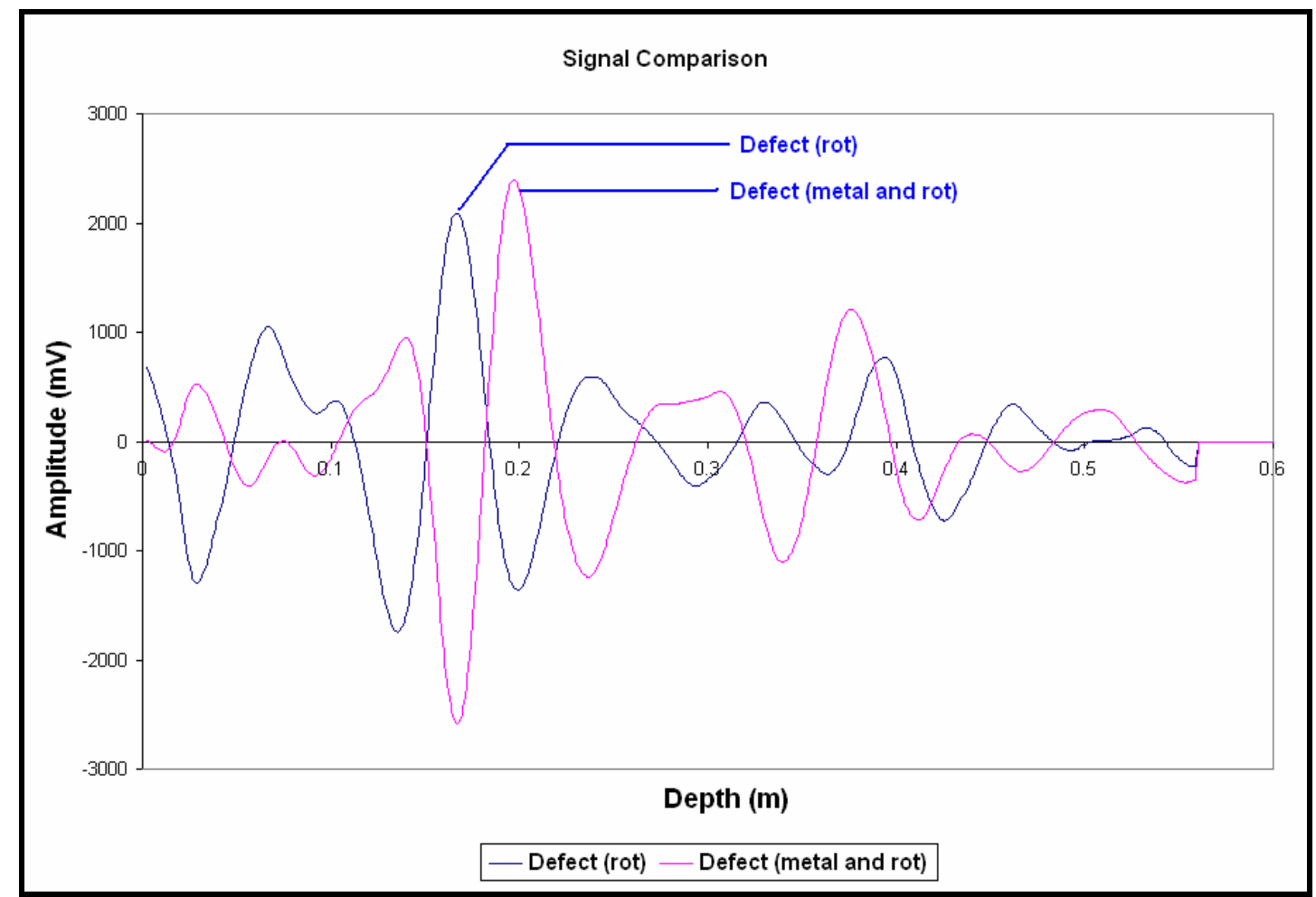

(c)

Figure 5-37 Comparison of GPR waveforms from sections with defects (rot and metal) in Log 3 for (a) raw data (Amplitude vs. Time), (b) processed data (Amplitude vs. Time), and (c) processed data (Amplitude vs. Depth)

\subsection{LOG 4}

Table 5-4 Characteristics of Log 4

\begin{tabular}{|c|c|c|c|c|c|c|c|c|}
\hline \multirow{2}{*}{$\begin{array}{c}\text { Log } \\
\text { no. }\end{array}$} & Species & $\begin{array}{c}\text { Length } \\
(\mathbf{m})\end{array}$ & $\begin{array}{c}\text { Butt } \\
\text { end }\end{array}$ & $\begin{array}{c}\text { Small } \\
\text { end }\end{array}$ & Surface & Max. & Average & Dielectric \\
Constant \\
\hline 4 & $\begin{array}{c}\text { Yellow } \\
\text { Poplar }\end{array}$ & 2.92 & 0.3 & 0.28 & 8 & 22.3 & 16 & 5.3 \\
\hline
\end{tabular}

The characteristics of Log 4 are given in Table 5-4 and the log is shown in Figure 5-38(a). Seven standard metal rods of $0.0095 \mathrm{~m} \mathrm{(3/8} \mathrm{in)} \mathrm{diameter} \mathrm{and} 0.09 \mathrm{~m} \mathrm{(31/2} \mathrm{in)}$ length were inserted in different orientations (some inserted from the top of the log and 
some from the side) as shown in Figure 5-38(b). This figure does not show the hole for the last metal, which was close to the small end of the log. In addition to the holes for the inserted metal rods, Figure 5-38(b) also shows an empty hole of $0.0095 \mathrm{~m} \mathrm{(3/8}$ in) diameter. The actual spacing between the inserted metal rods is given in the second column of Table 5.5. A single antenna positioned at the top of the log, as shown in Figure 5-39, was used for collecting the data. The user defined data collection and processing parameters are shown in Figure 5-40.

The process of data analysis for $\log 4$ was similar to that for $\log 1$. The raw GPR data for $\log 4$ is shown in Figure 5-41. The top set of white-black-white band is due to surface coupling. Another set of black and white band is visible at $5.8 \mathrm{~ns}$ range, representing the reflection from the bottom of the log. In the bottom reflection, black band was seen first as compared to white band in the surface reflection. This is because the reflection of the bottom (wood-air) interface had a change of phase with respect to the top interface (antenna-wood) reflection. Also, in the raw GPR data in Figure 5-41(b), the locations of 4 metal rods at distances of $0.5 \mathrm{~m}, 1.00 \mathrm{~m} 1.55 \mathrm{~m}$, and $1.9 \mathrm{~m}$ could be easily predicted, since the corresponding signal clutters (reflection echoes) have parabolic shapes which indicate the presence of defects having high reflection coefficient.

The data was processed to locate other metal defects not visible in the raw GPR data in Figure 5-41. After the data was processed, the metal rods were located based on parabolic shaped signal clutter in the processed GPR scan shown in Figure 5-42. From the processed data, location of additional metal rods could be easily predicted.

The wiggle plots for the raw and the processed GPR scans are shown in Figure 543. The waveforms in these wiggle plots were of very little use in understanding and locating the defects. This is because the wiggle plots are made after averaging 16 signals to obtain one signal, and the amplitudes can not be increased beyond a certain point because of the low spacing between the signals. On the other hand, the grey scale scans in Figure 5-42 represent amplitudes in terms of intensities of the band, thus making them easier to interpret.

The processed GPR scan in Figure 5-42(b) and the corresponding metal defect locations along the length of the log are shown as a combined picture in Figure 5-44. The 
GPR scan in Figure 5-44 shows the location of the embedded metal rods (small cylindrical defect with high reflection coefficient) as distinct signal clutters with parabolic shape. The center of the parabolas represents the defect locations, and the predicted spacings are tabulated in the second column of Table 5-5. It can be seen from Table 5-5 that the predicted distance locations along the length of the log were all within $0.02 \mathrm{~m}$ of the actual locations.

Table 5-6 shows a comparison of the predicted depths for the subsurface metals. The GPR predictions are within $0.03 \mathrm{~m}$ of the actual depths. Since the reflection from Metal 1 was outside the GPR scan, no data is reported for Metal 1 in Tables 5-5 and 5-6.

The GPR scan (Figure 5-44) did not show the empty hole of $0.0095 \mathrm{~m} \mathrm{(3/8}$ in)

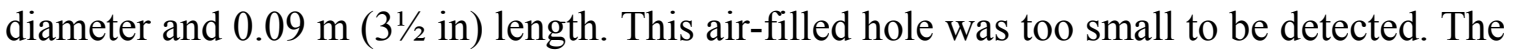
subsurface defects such as knots and rots are usually of larger diameters and filled with a significant amount of moisture, thus making them detectable unlike the empty hole that was drilled into this log.

From the raw and the processed GPR scans, individual waveforms for defective sections were extracted and compared with a waveform from a defect-free section. The waveform comparison is shown in Figure 5-45(a) for raw data and Figures 5-45(b) and 545(c) for processed data. The vertical axis for all the waveforms in Figure 5-45 show the waveform amplitude and the horizontal axis shows either two-way travel time or the depth $(\mathrm{m})$ through the log. The raw waveforms in Figure 5-45(a) show very little differences between them. On the other hand, the processed data in Figure 5-45(b) and 545(c) showed several key features. From these figures, the location of the embedded metal rods along the depth of the log could be predicted depending on the position of high positive amplitude signals. It can seen from signal comparison in Figure 5-45(c) that metal numbers 3, 4, and 7 are located at depths ranging from 0.06 to $0.09 \mathrm{~m}$.

There was an excellent correlation between the predicted and the actual locations of the metal defects along the length of the log. The predicted and actual depths also correlated equally well. 


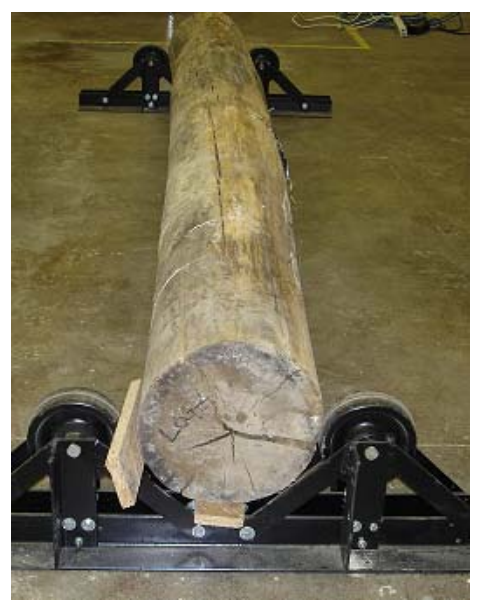

(a)

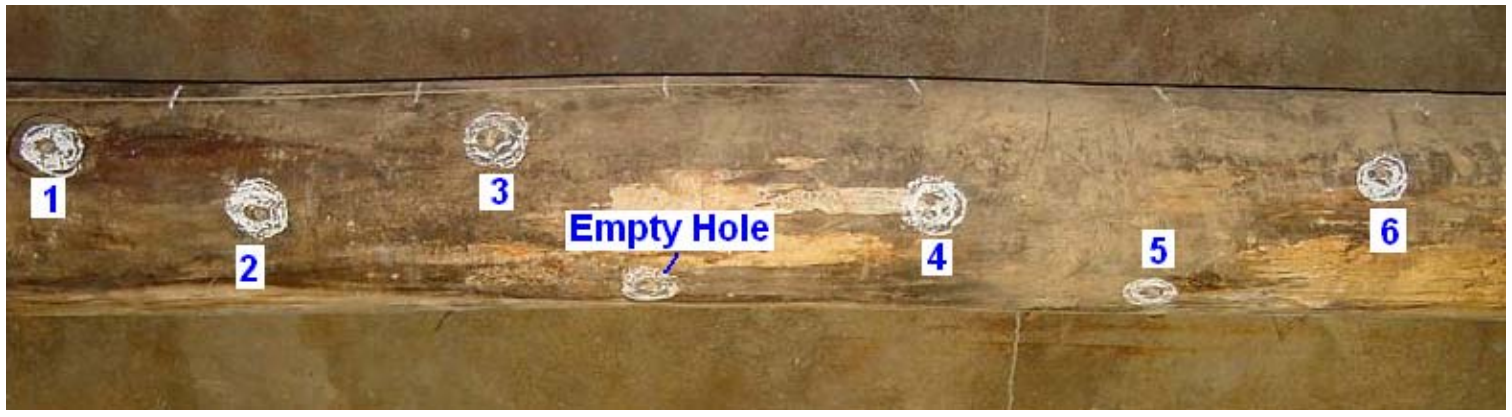

(b)

Figure 5-38 Picture of Log 4 showing the locations where metal rods were inserted

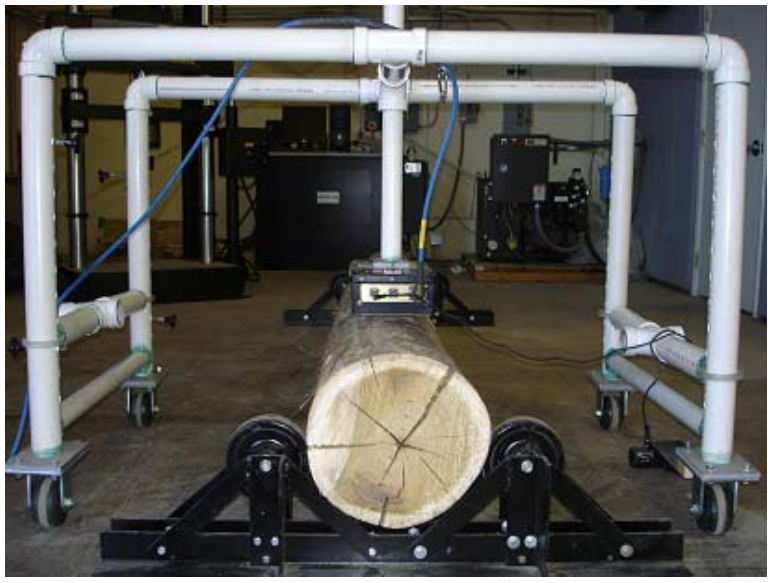

Figure 5-39 Antenna configuration 


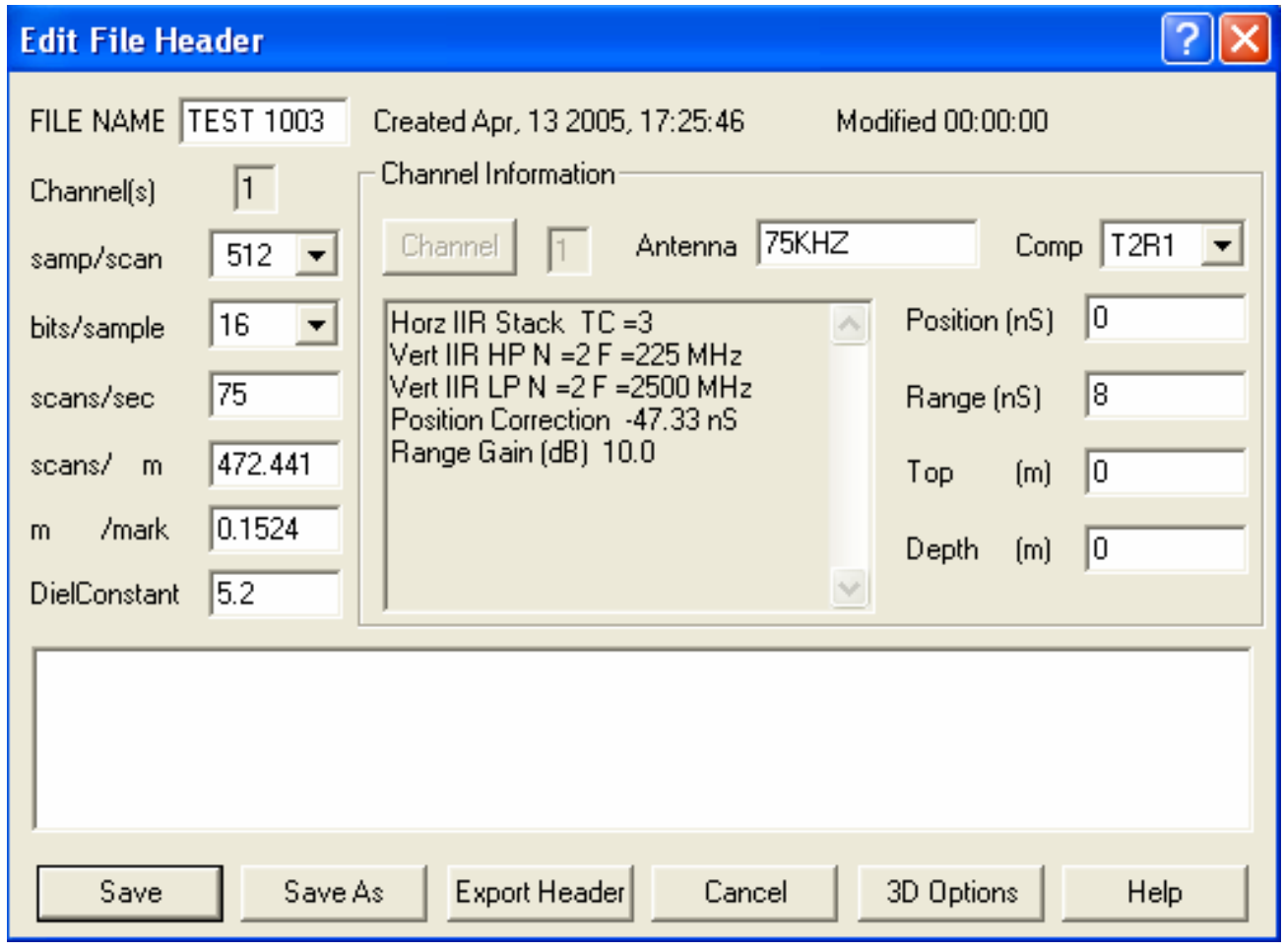

(a)

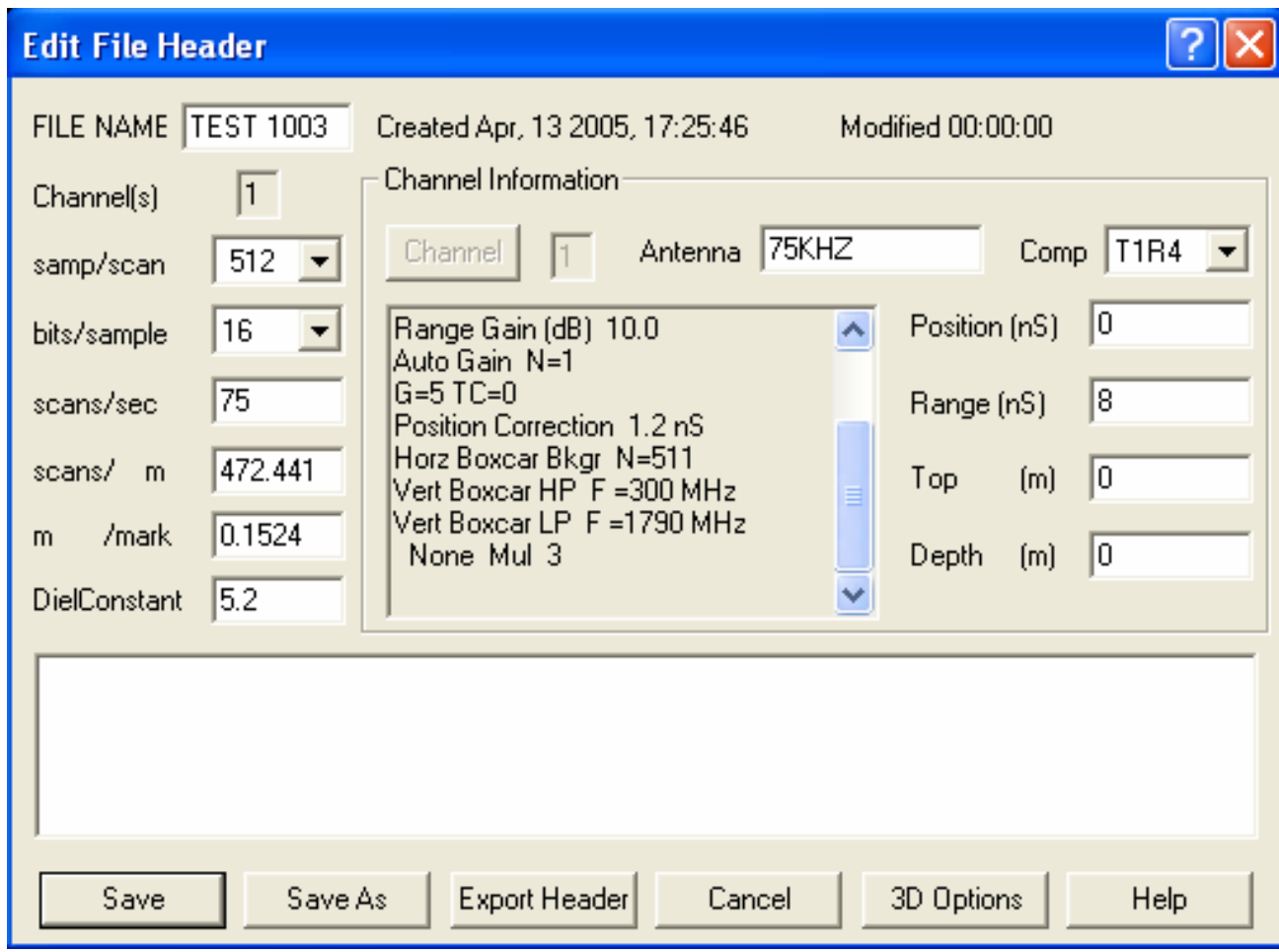

(b)

Figure 5-40 (a) Data collection parameters, (b) data processing parameters 


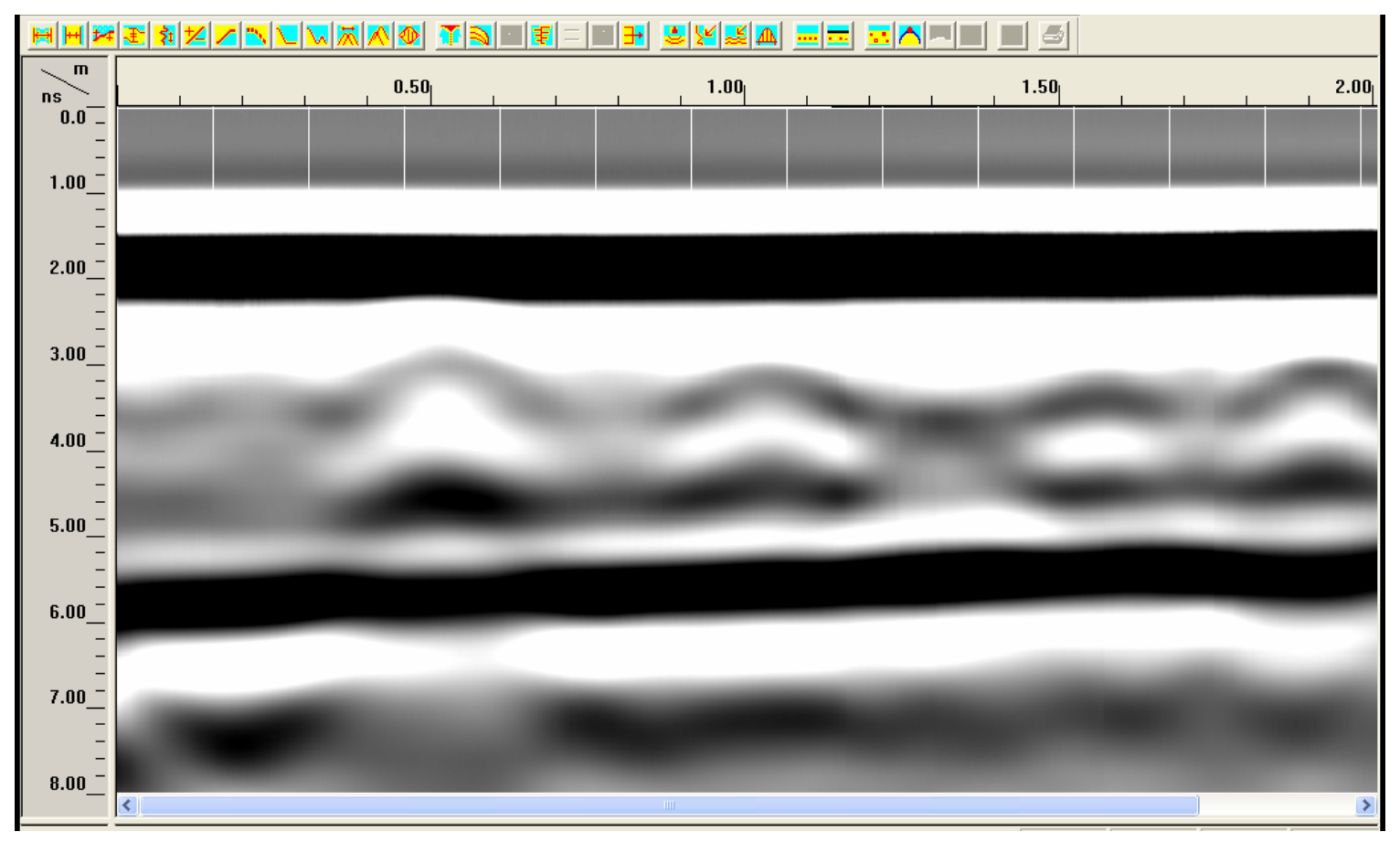

(a)

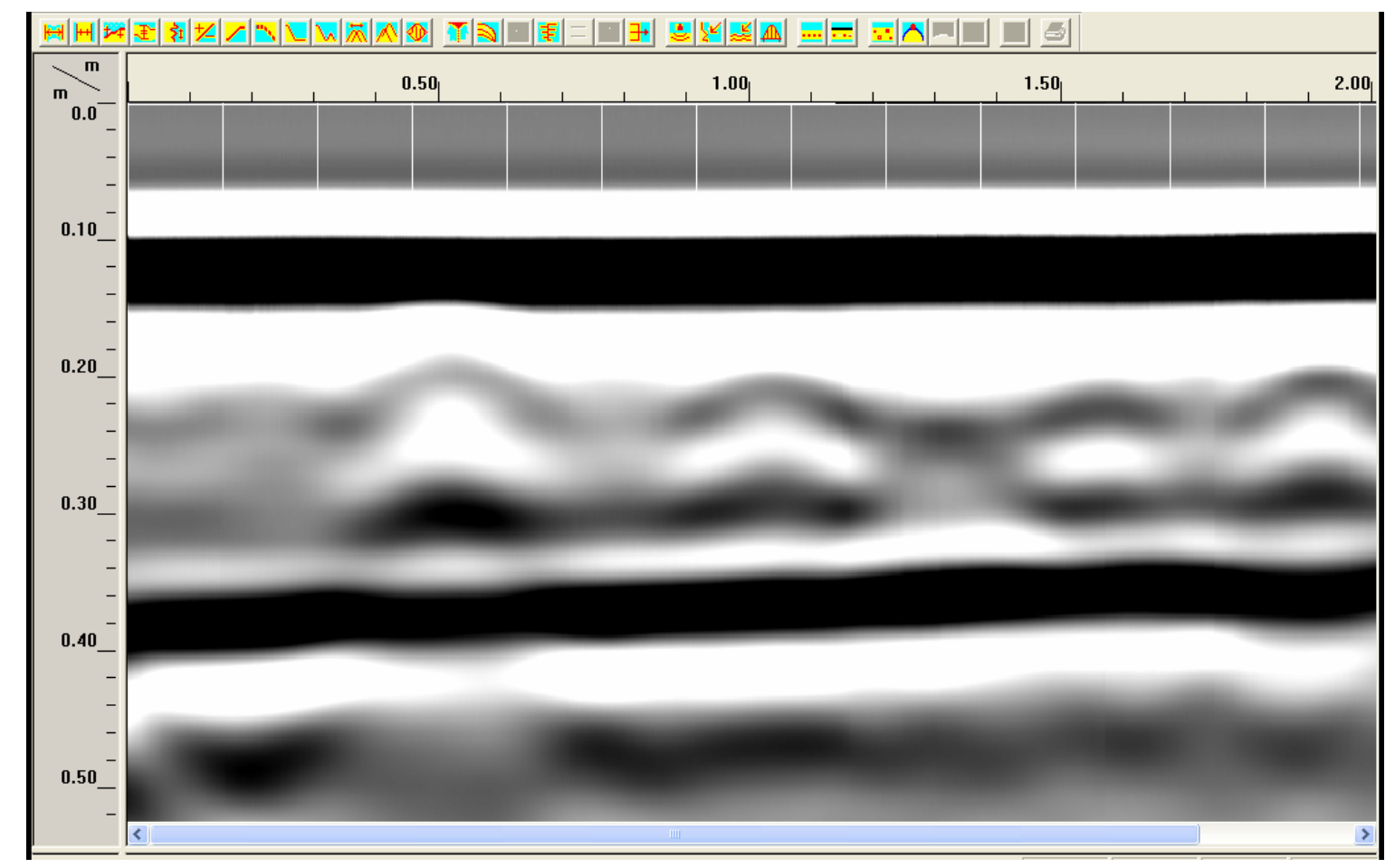

(b)

Figure 5-41 Raw GPR data of Log 4, horizontal scale indicating the travel distance along the length of the $\log (\mathrm{m})$ and the vertical scale indicating (a) time (ns) (b) depth (m) 


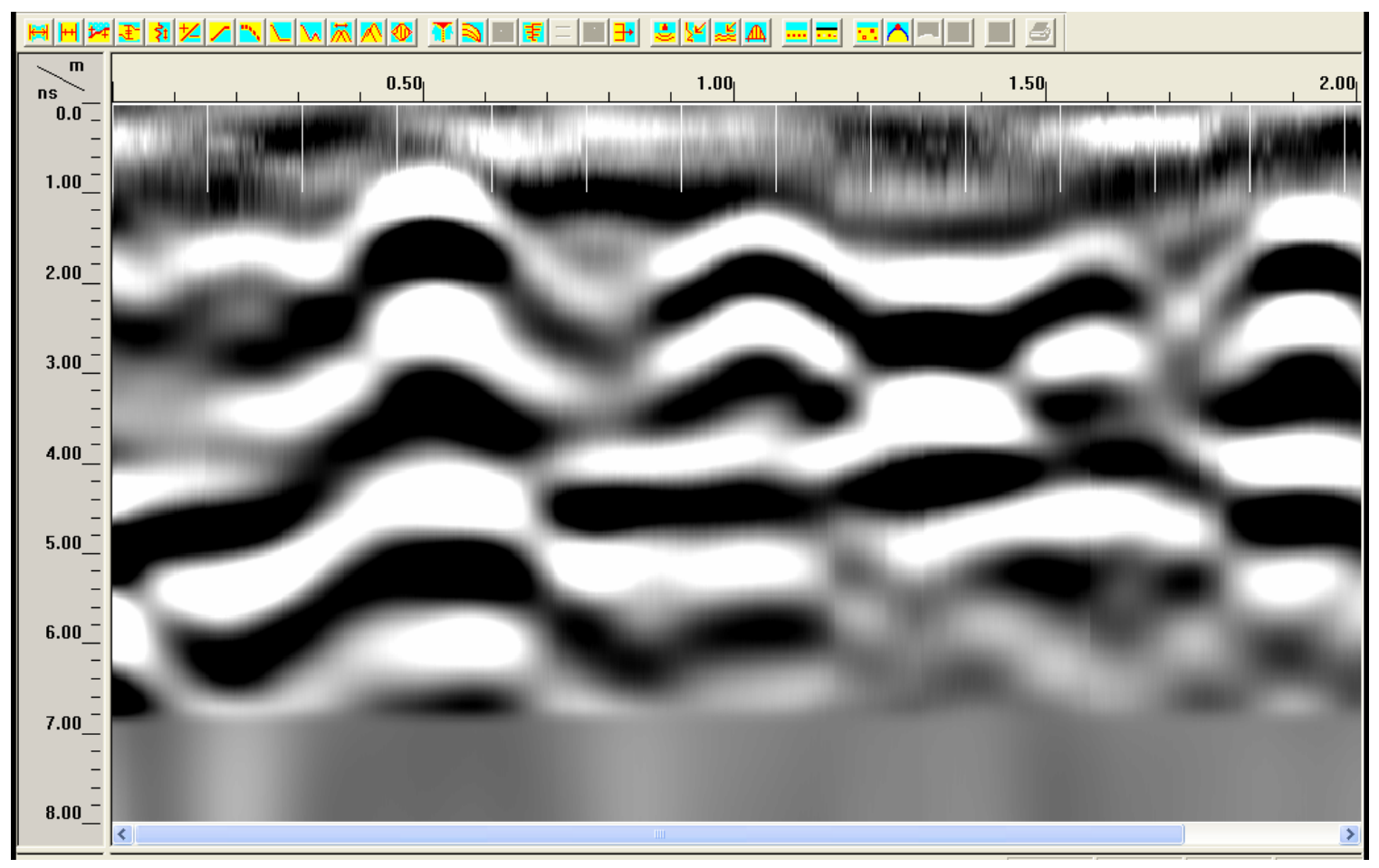

(a)

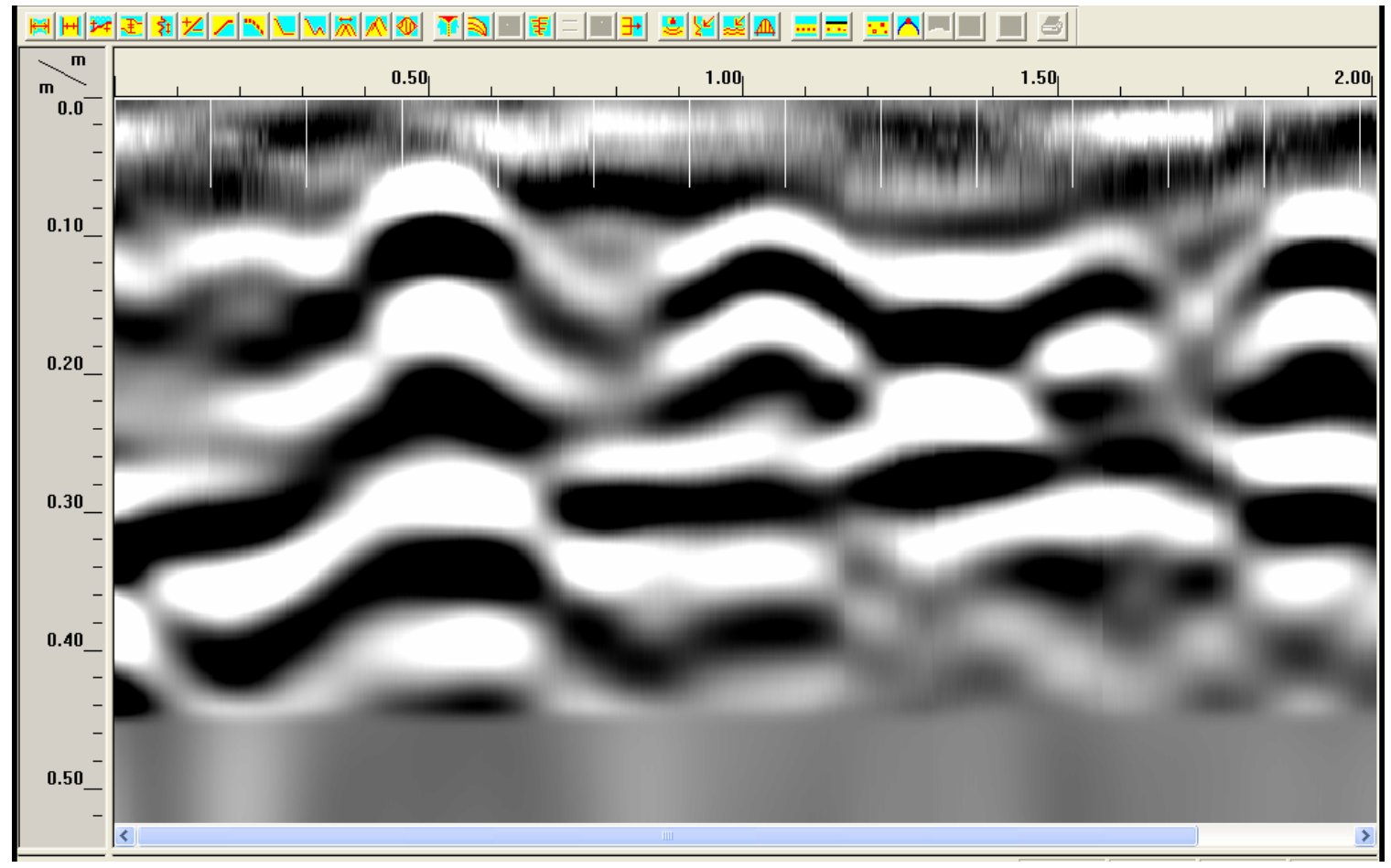

(b)

Figure 5-42 Processed GPR data of $\log 4$, horizontal scale indicating the travel distance along the length of the $\log (\mathrm{m})$ and the vertical scale indicating (a) time (ns) (b) depth (m) 


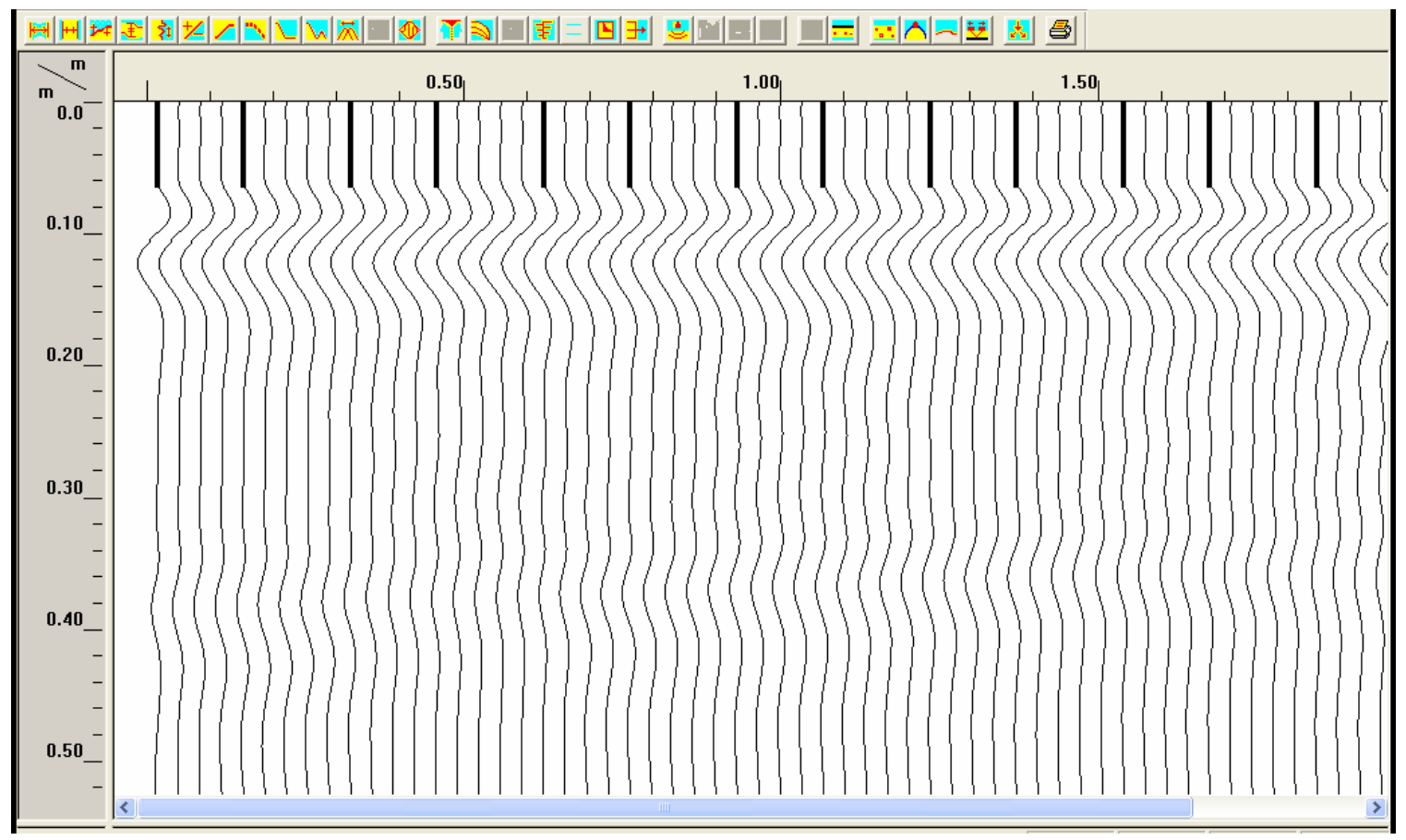

(a)

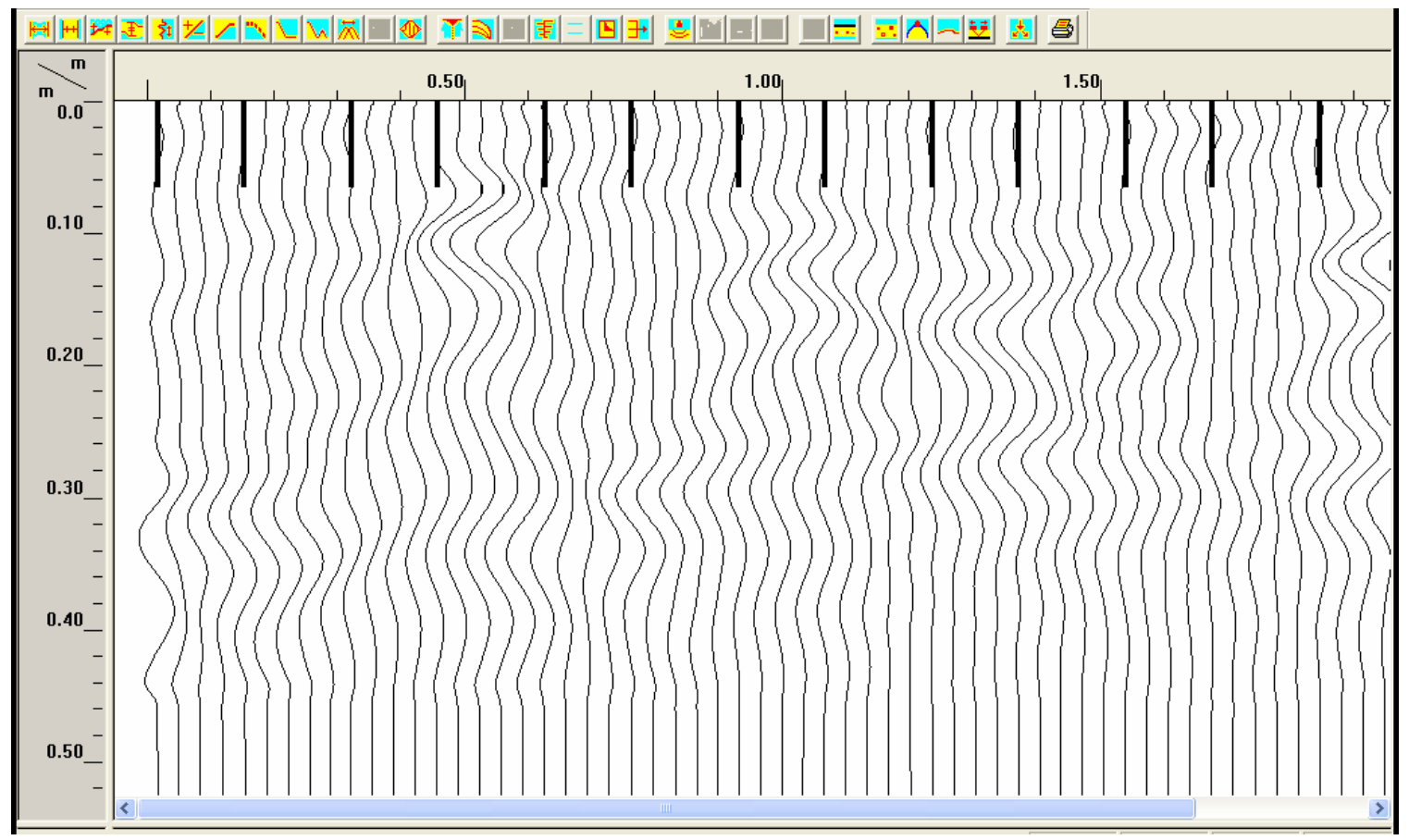

(b)

Figure 5-43 Wiggle plot of $\log 4$, horizontal scale indicating travel distance along the length of the $\log (\mathrm{m})$ and vertical scale indicating the depth (m), for (a) raw data, and (b) processed data 


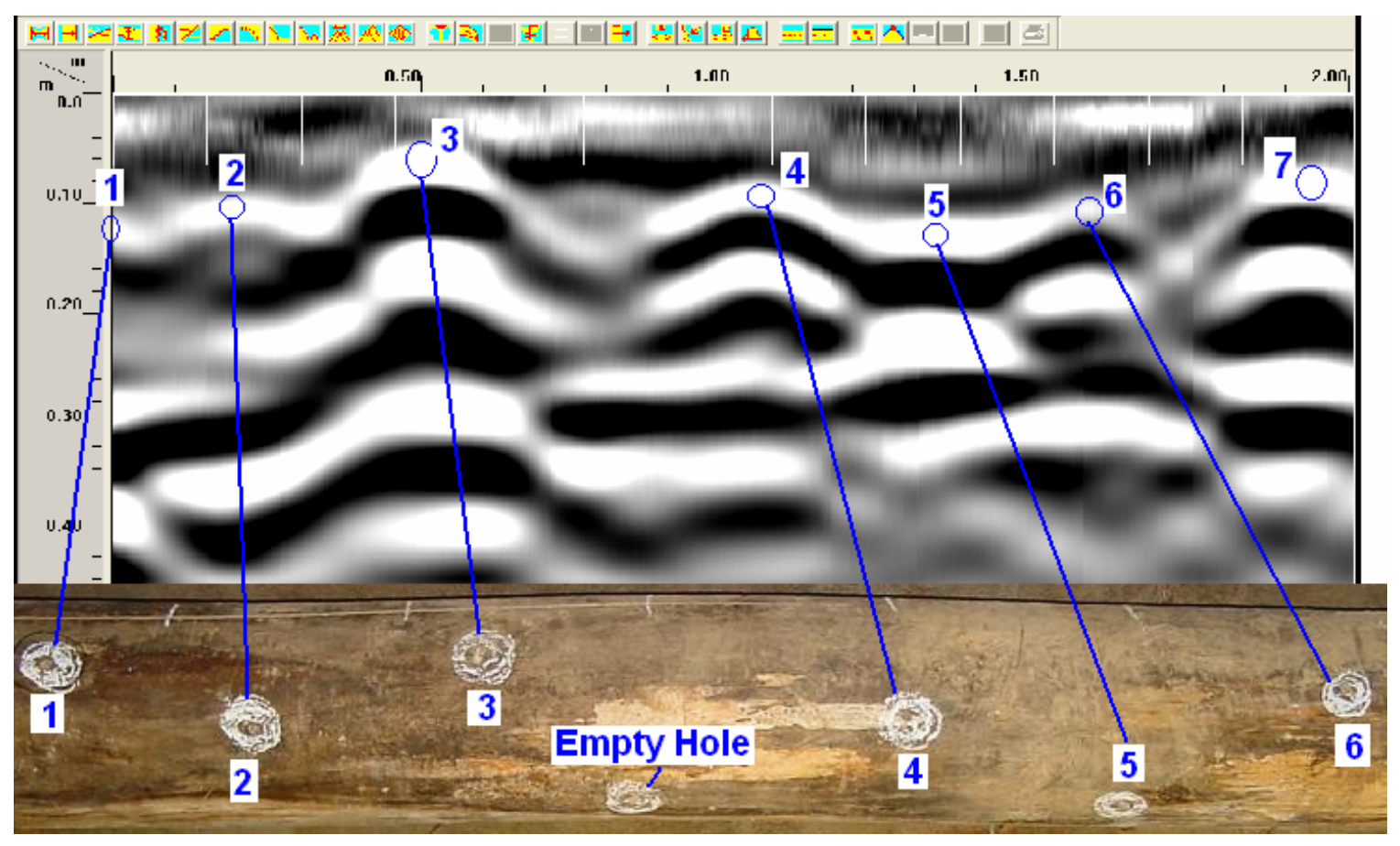

Figure 5-44 Processed GPR data and corresponding locations where metal rods were inserted

Table 5-5 Comparison between actual and predicted spacing between metals

\begin{tabular}{|c|c|c|}
\hline \multirow{2}{*}{ Between Metals } & \multicolumn{2}{|c|}{ Spacing (m) } \\
\hline & Actual & Predicted by GPR \\
\hline $1-2$ & 0.27 & - \\
\hline $2-3$ & 0.28 & 0.30 \\
\hline $3-4$ & 0.52 & 0.54 \\
\hline $4-5$ & 0.29 & 0.30 \\
\hline $5-6$ & 0.26 & 0.24 \\
\hline $6-7$ & 0.40 & 0.38 \\
\hline
\end{tabular}


Table 5-6 Comparison between actual and predicted depth for metals

\begin{tabular}{|c|c|c|}
\hline \multirow{2}{*}{ Metal No. } & \multicolumn{2}{|c|}{ Depth (m) } \\
\cline { 2 - 3 } & Actual & Predicted by GPR \\
\hline 1 & 0.05 & 0.11 \\
\hline 2 & 0.10 & 0.06 \\
\hline 3 & 0.05 & 0.09 \\
\hline 4 & 0.10 & 0.14 \\
\hline 5 & 0.17 & 0.11 \\
\hline 6 & 0.10 & 0.08 \\
\hline 7 & 0.10 & \\
\hline
\end{tabular}

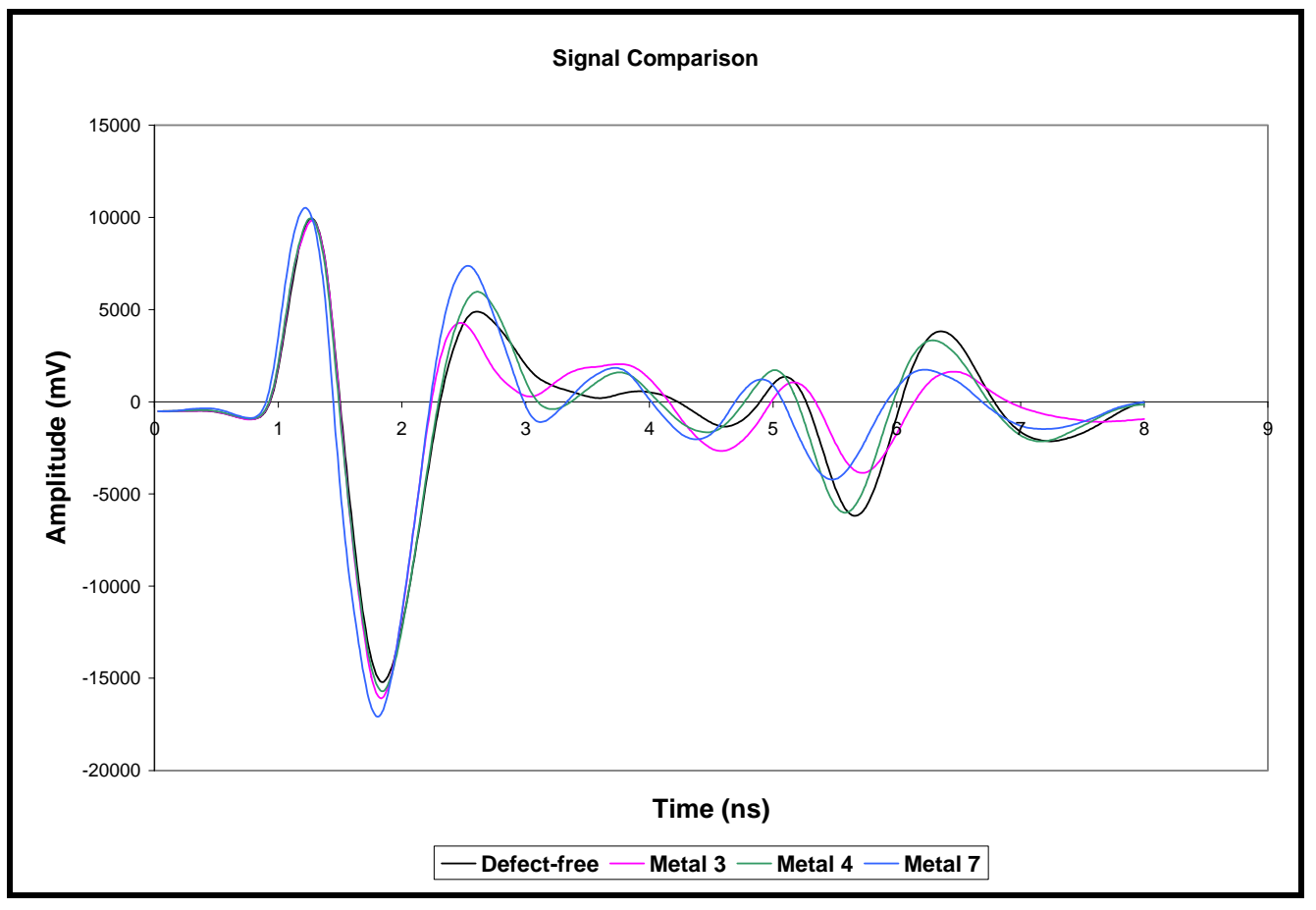

(a) 


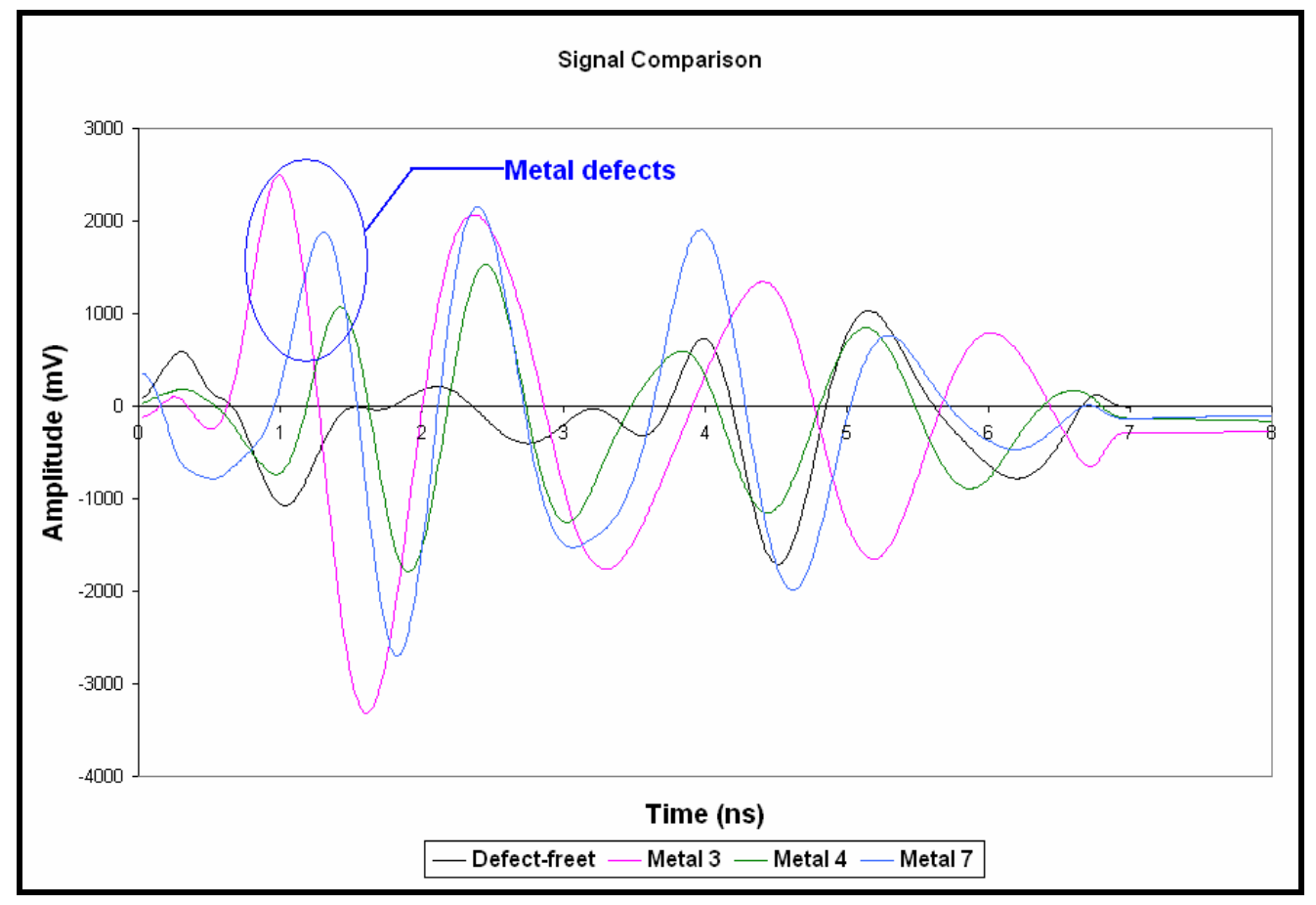

(b)

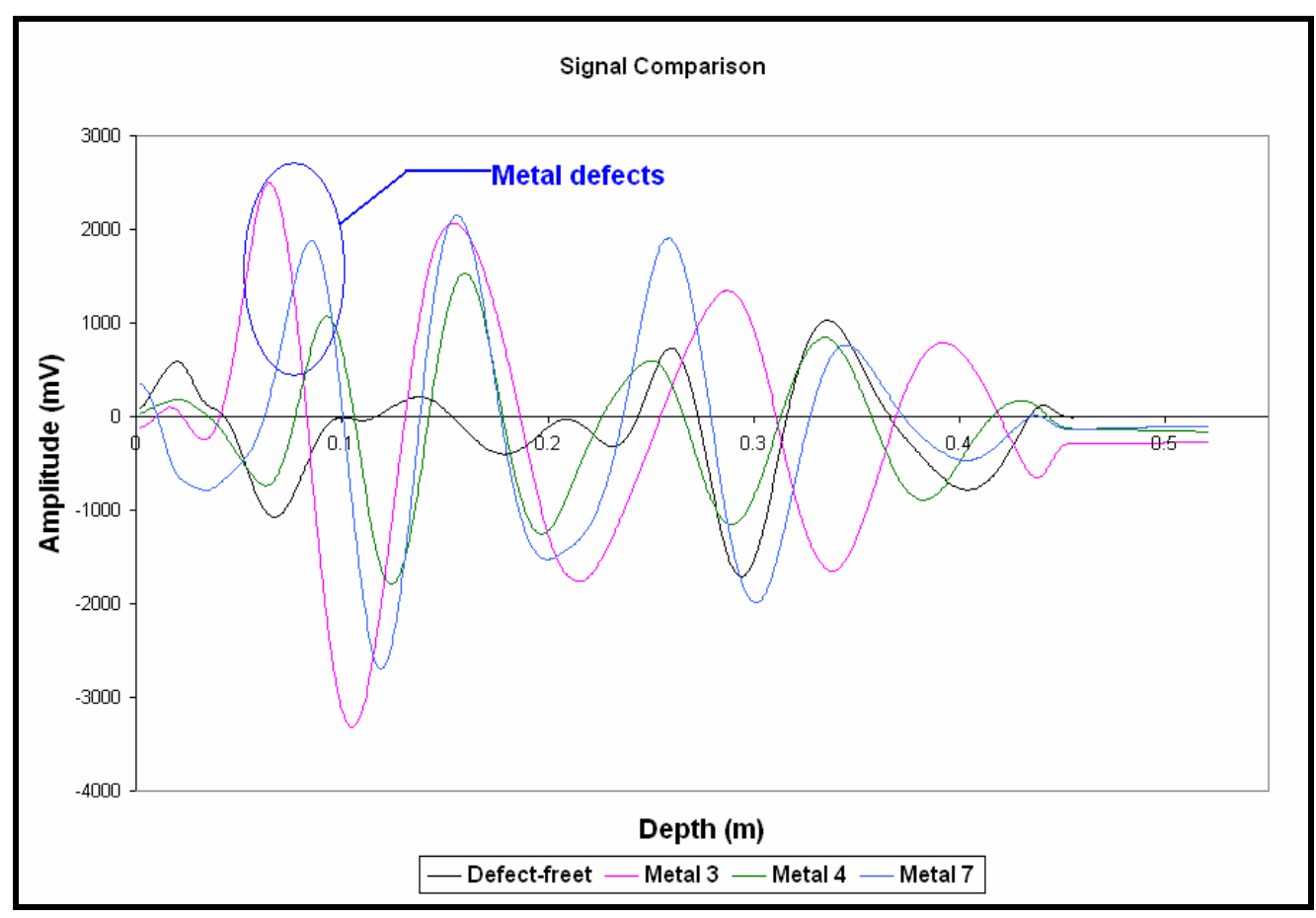

(c)

Figure 5-45 Comparison of GPR waveforms from sections with and without defects (metal rods) in Log 4 for (a) raw data (Amplitude vs. Time), (b) processed data (Amplitude vs. Time), and (c) processed data (Amplitude vs. Depth) 


\section{$5.5 \quad$ LOG 5}

Table 5-7 Characteristics of $\log 5$

\begin{tabular}{|c|l|c|c|c|c|c|c|c|}
\hline $\begin{array}{c}\text { Log } \\
\text { no. }\end{array}$ & Species & $\begin{array}{c}\text { Length } \\
\text { (m) }\end{array}$ & $\begin{array}{c}\text { Diameter (m) } \\
\text { Butt } \\
\text { end }\end{array}$ & $\begin{array}{c}\text { Small } \\
\text { end }\end{array}$ & Surface & Max. & Average & $\begin{array}{c}\text { Dielectric } \\
\text { Constant }\end{array}$ \\
\hline 5 & $\begin{array}{c}\text { Yellow } \\
\text { Poplar }\end{array}$ & 2.98 & $\begin{array}{c}0.48 \\
\text { to }\end{array}$ & $\begin{array}{c}0.38 \\
\text { to }\end{array}$ & 22.9 & 99 & 35.4 & 12 \\
\hline
\end{tabular}

The characteristics of $\log 5$ are shown in Table 5-7. The log scanning setup is shown in Figure 5-46. Figure 5-47 shows the butt end of the log placed on the sawing machine ready to be sawn. This log had a very non-uniform diameter as shown in Figure47. The variation in diameters at the butt and small ends of the log are shown in Table 57. The moisture content in the center of the $\log$ was very high $(99 \%)$ and the average moisture content was $35.4 \%$ as shown in Table 5-7. The data collection and processing parameters for $\log 5$ are shown in Figure 5-48. These parameters are explained in Sections 4-1 and 4.2. Total of six GPR scans were collected by rotating the log.

The process of data analysis for this $\log$ was similar to that for Log 1. Figure 5-49 shows one of the raw GPR scans (scan \# 5) with horizontal scale showing the distance traveled by the GPR antenna along the surface of the log. The top sets of white-blackwhite band were due to surface coupling between the antenna and the log. This band is immediately followed by another set of white-black band which indicates a high moisture zone just below the surface. Echoes from the bottom of the log show up as bands arriving later in time $(\sim 12 \mathrm{~ns}$ or $0.5 \mathrm{~m})$ which are also of lower intensity due to signal attenuation and reflection/transmission losses within the log. From the raw data in Figure 5-49 it was difficult to predict any internal features (defects).

The processed GPR scan for scan \# 5 is shown in Figure 5-50. It can be seen that the time and depth axis in this figure have all shifted upwards in comparison to Figure 549 because of zero correction. The bottom of the log was at $11.5 \mathrm{~ns}(0.48 \mathrm{~m})$ near the 
start of the scan and $10 \mathrm{~ns}(0.44 \mathrm{~m})$ near the end of the scan. The high amplitude signal clutter at $3.75 \mathrm{~ns}$ or $0.15 \mathrm{~m}$ depth was either due to high moisture content or due to presence of internal features. These signal clutters were marked as shown in Figure 5-50. The wiggle plot of raw (Figure 5-49(b)) and the processed (Figure 5-50(b)) GPR scans are shown in Figure 5-51(a) and 5-51(b), respectively. However, it was very difficult to predict any internal feature from the wiggle traces. Therefore, the internal features were predicted and marked based on the processed GPR scans in Figure 5-52, which shows all six GPR scans around the log. As it can be seen from this figure, all the defects were located nearly in the center core of the log between $3.5 \mathrm{~ns}(0.15 \mathrm{~m})$ and $7.5 \mathrm{~ns}(0.3 \mathrm{~m})$.

To validate the prediction the log was sawn assuming that there was no embedded metal. The $\log$ was sawn into 1 in $(0.0254 \mathrm{~m})$ thick horizontal planks, and the corresponding top antenna viewing position was that for scan 5. During sawing of Log 5, the saw blade came in contact with an embedded metal nail shown in Figure 5-53. This metal piece had $0.0254 \mathrm{~m}$ length and $0.004 \mathrm{~m}$ diameter, and caused damage to the saw blade. The metal defect was located at a depth of $0.26 \mathrm{~m}$ and a distance of $0.6 \mathrm{~m}$ from the start of the scan (the butt end). Due to its small size, this embedded metal was not detected in the GPR scans. There was significant amount of downtime ( $\sim 1$ hour) involved in replacing the damaged saw blade. This problem is shown in pictorial form in Figure 554. After sawing the log it was seen that there were no significant internal defects except the embedded metal. The knots detected were of very small in size $(\sim 0.03 \mathrm{~m}$ diameter $)$ and were present in central core of the log. Some of the knots observed after sawing are shown in Figure 5-55. All the defects were found to be located at a depth range of $0.15 \mathrm{~m}$ to $0.3 \mathrm{~m}$.

Figure 5-56 shows the actual defects observed in Log 5 after sawing and the corresponding signal clutters in GPR scan \#5. There is a good correlation between the marked signal clutters and the observed defects, except that the GPR scans show few additional signal clutters which were not really defects and could simply be high moisture pockets. The actual defects, which were all knots, were numbered as shown in Figure 556. The actual location of knot 1 was at $0.24 \mathrm{~m}$ depth and $0.4 \mathrm{~m}$ distance from the butt end) as compared to the predicted location of $0.2 \mathrm{~m}$ and $0.4 \mathrm{~m}$. Knot 2 was located at a depth of $0.2 \mathrm{~m}$ and at a distance of $1.2 \mathrm{~m}$, which compared well with the predicted depth 
of $0.2 \mathrm{~m}$ and $1.22 \mathrm{~m}$. Knot 3 was located at a depth of $0.27 \mathrm{~m}$ and a distance of $1.53 \mathrm{~m}$, which compared well with the predicted depth of $0.25 \mathrm{~m}$ and a distance of $1.53 \mathrm{~m}$. Knot 4 was located at a depth of $0.27 \mathrm{~m}$ and a distance of $1.8 \mathrm{~m}$, which compared well with the predicted depth of $0.27 \mathrm{~m}$ and a distance of $1.8 \mathrm{~m}$. Knot 5 was located at a depth of 0.23 $\mathrm{m}$ and distance of $2.2 \mathrm{~m}$, which compared well with the predicted depth of $0.26 \mathrm{~m}$ and a distance of $2.2 \mathrm{~m}$. Knot 6 was located at a depth of $0.24 \mathrm{~m}$ and a distance of $2.4 \mathrm{~m}$, which compared will with the predicted depth of $0.25 \mathrm{~m}$ and a distance of $2.4 \mathrm{~m}$. All the above actual and predicted values are listed in Table 5-8, which shows that the GPR resolution was $0.04 \mathrm{~m}$ for depth and $0.02 \mathrm{~m}$ for distance along the length of the log.

From the raw and processed GPR scans, the individual waveforms for defective sections were extracted and compared with a waveform from a defect-free section. The waveform comparison is shown in Figure 5-57. The raw waveform shown in Figure 57(a) show very little difference between the signals. On the other hand, the processed data in Figure 5-57(b) and 5-57(c) shows several prominent peaks. From the graph knot 4 was located at $0.27 \mathrm{~m}(6 \mathrm{~ns})$ and knot 6 at a depth of $0.25 \mathrm{~m}(5.6 \mathrm{~ns})$ exactly as predicted from the GPR scan shown in Figures 5-50 and 5-56.

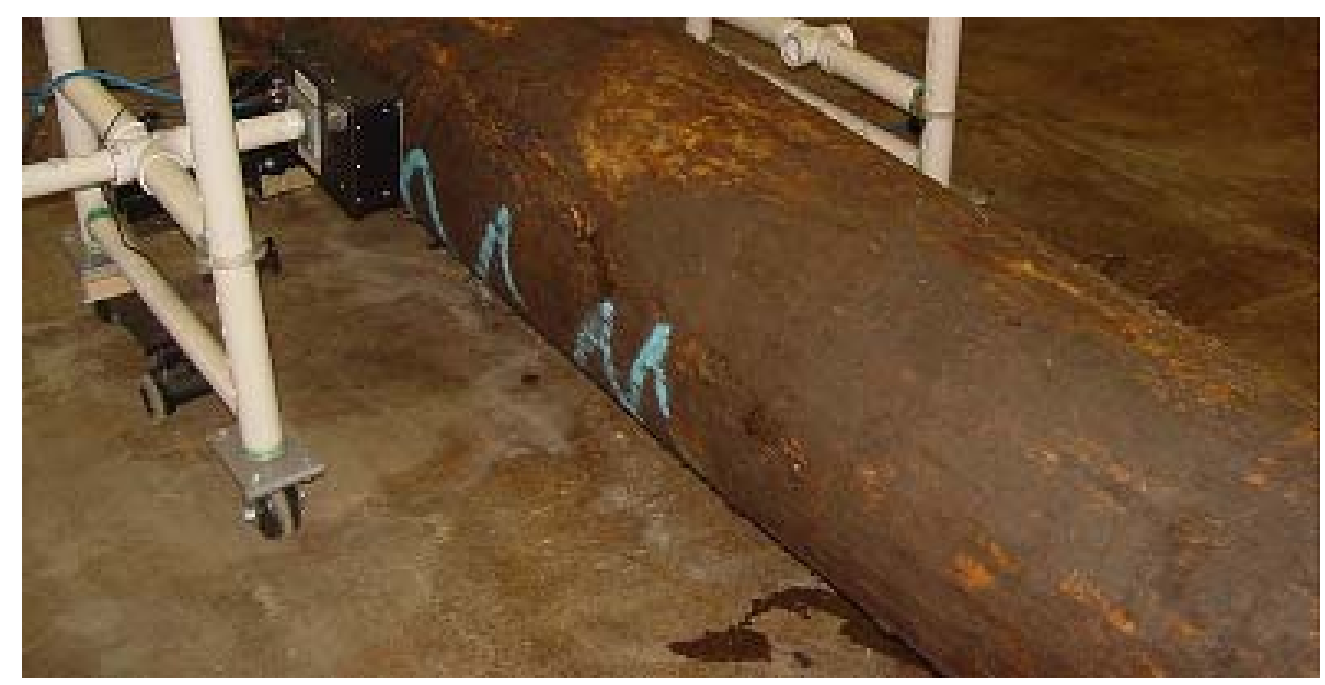

Figure 5-46 Log 5 and the antenna configuration used for collecting GPR data 


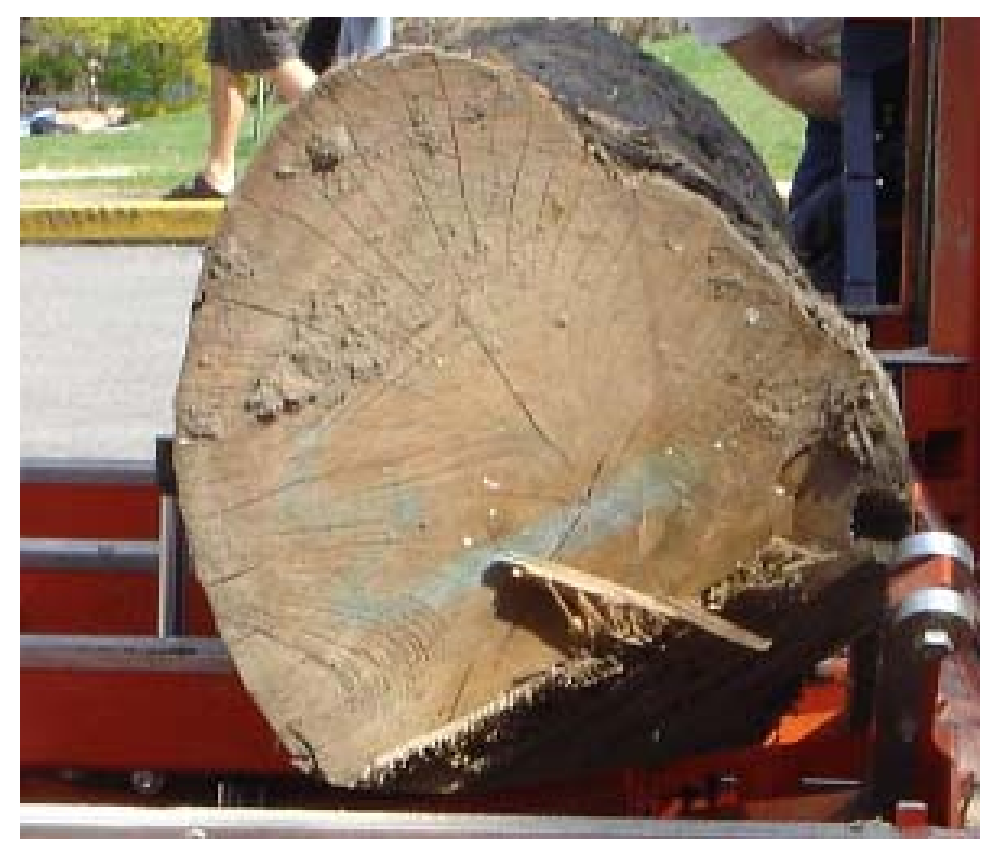

Figure 5-47 Picture showing butt end of $\log 5$

Table 5-8 Comparison between actual and predicted distance and depth for defects

\begin{tabular}{|c|c|c|c|c|}
\hline \multirow{2}{*}{ Defect No. } & \multicolumn{2}{|c|}{ Actual Location } & \multicolumn{2}{c|}{$\begin{array}{c}\text { Predicted Location Using } \\
\text { GPR }\end{array}$} \\
\cline { 2 - 5 } & Depth (m) & Distance (m) & Depth (m) & Distance (m) \\
\hline Knot 1 & 0.24 & 0.40 & 0.20 & 0.40 \\
\hline Knot 2 & 0.20 & 1.20 & 0.20 & 1.22 \\
\hline Knot 3 & 0.27 & 1.53 & 0.25 & 1.53 \\
\hline Knot 4 & 0.27 & 1.80 & 0.27 & 1.80 \\
\hline Knot 5 & 0.23 & 2.20 & 0.26 & 2.20 \\
\hline Knot 6 & 0.24 & 2.40 & 0.25 & 2.40 \\
\hline Metal & 0.26 & 0.60 & - & - \\
\hline
\end{tabular}




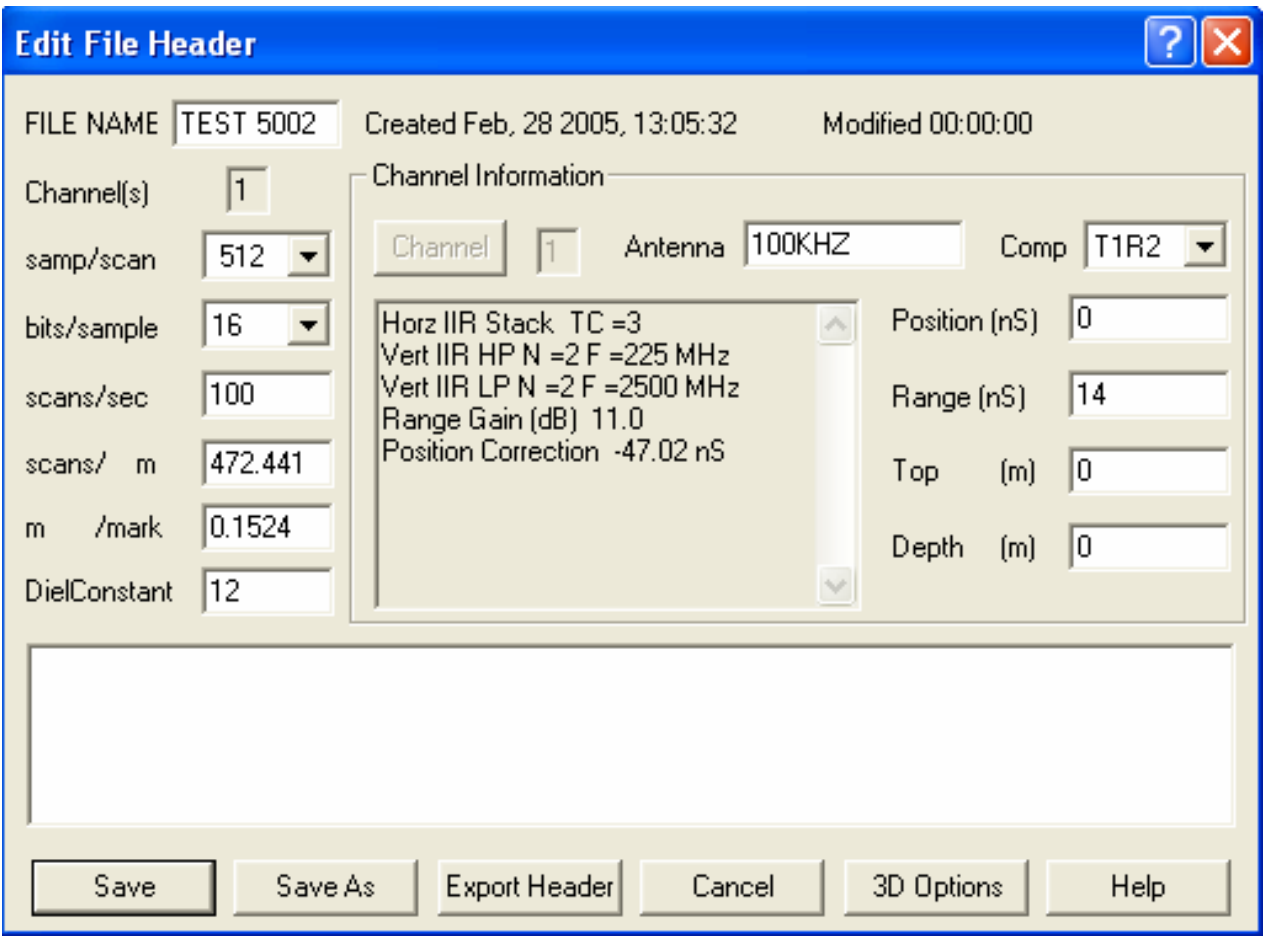

(a)

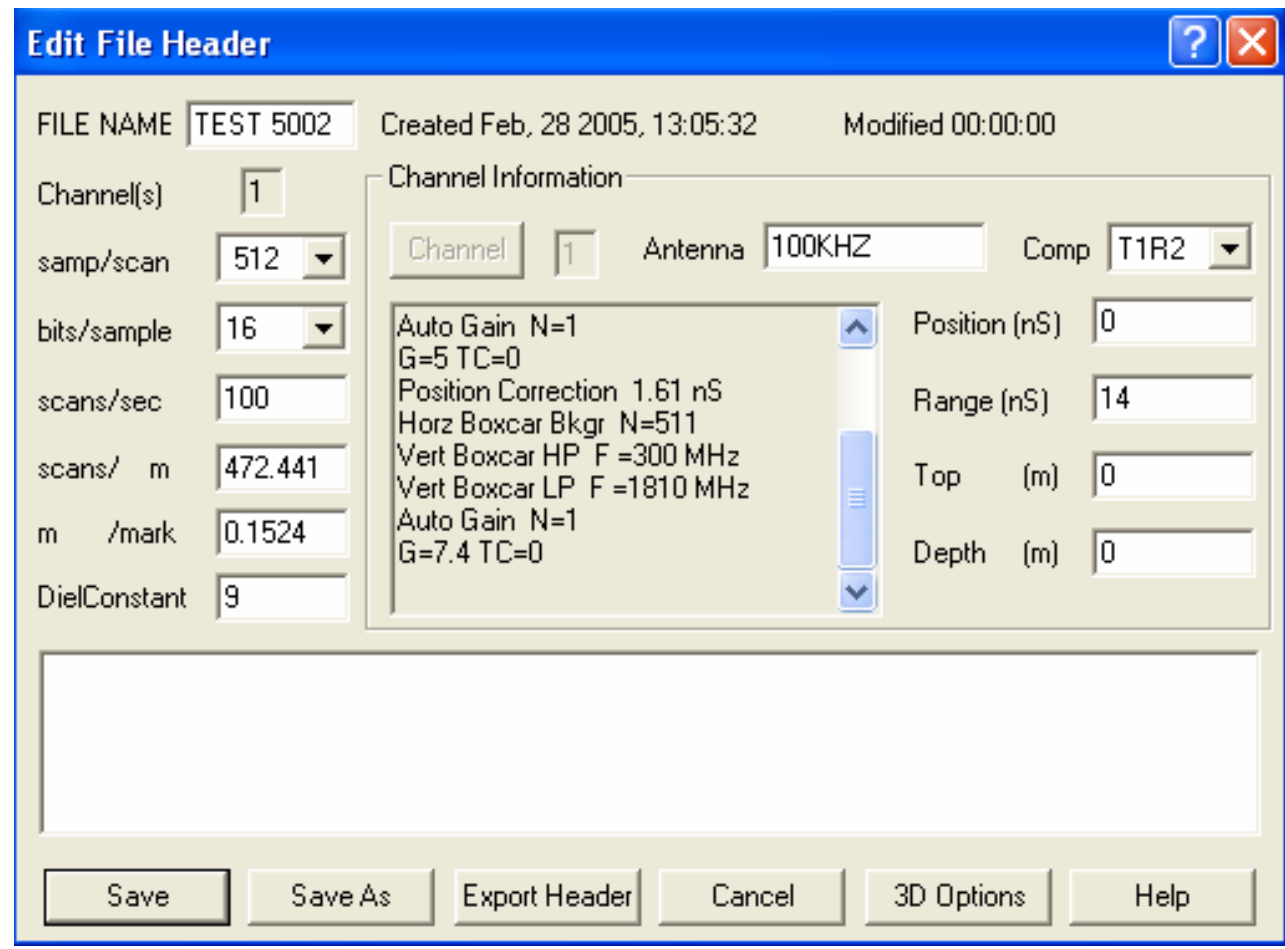

(b)

Figure 5-48 (a) Data collection parameters (b) data processing parameters 


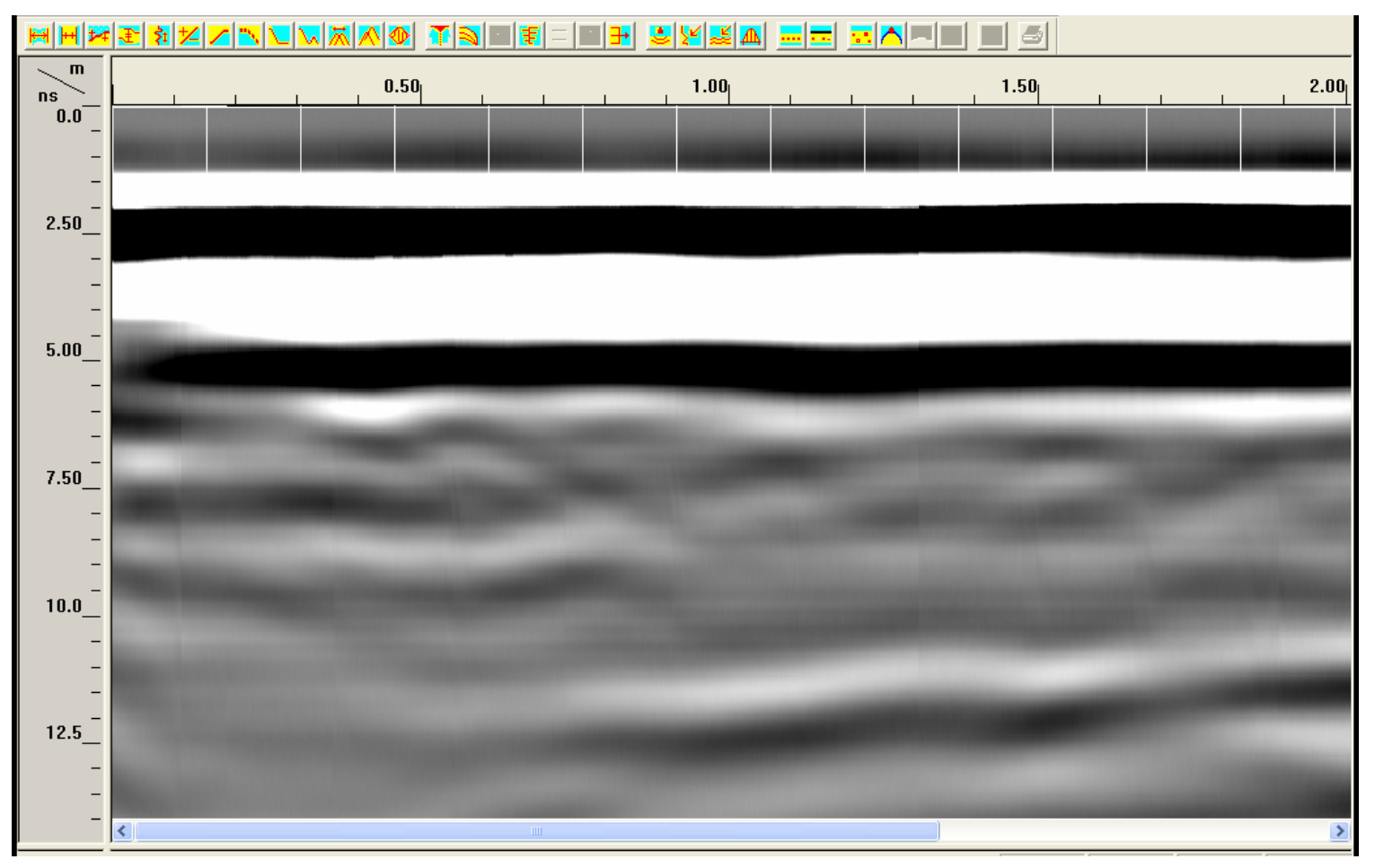

(a)

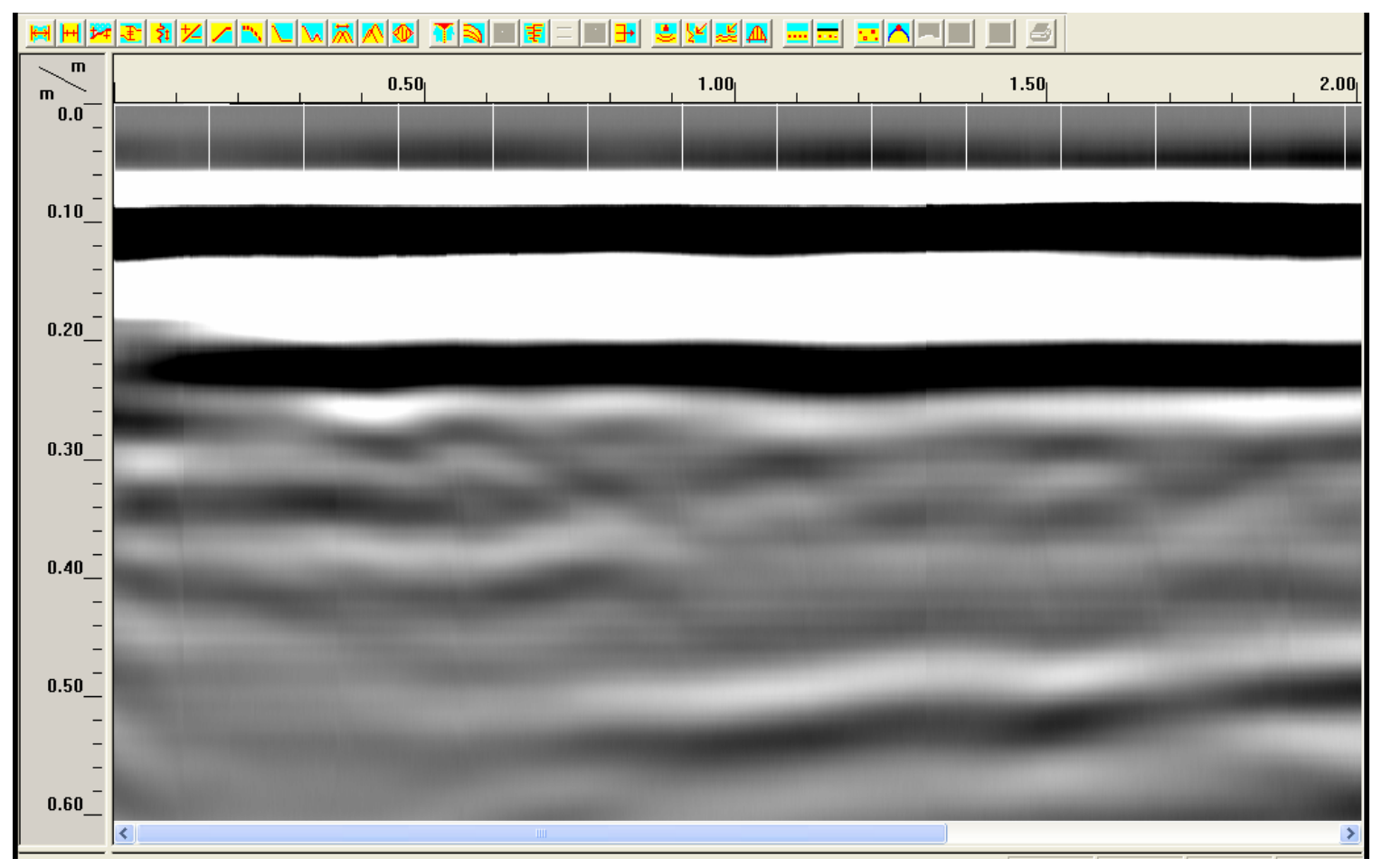

(b)

Figure 5-49 Raw GPR data of $\log 5$ (scan \# 5), horizontal scale indicating the travel distance along the length of the $\log (\mathrm{m})$ and the vertical scale indicating (a) time (ns) (b) depth (m) 


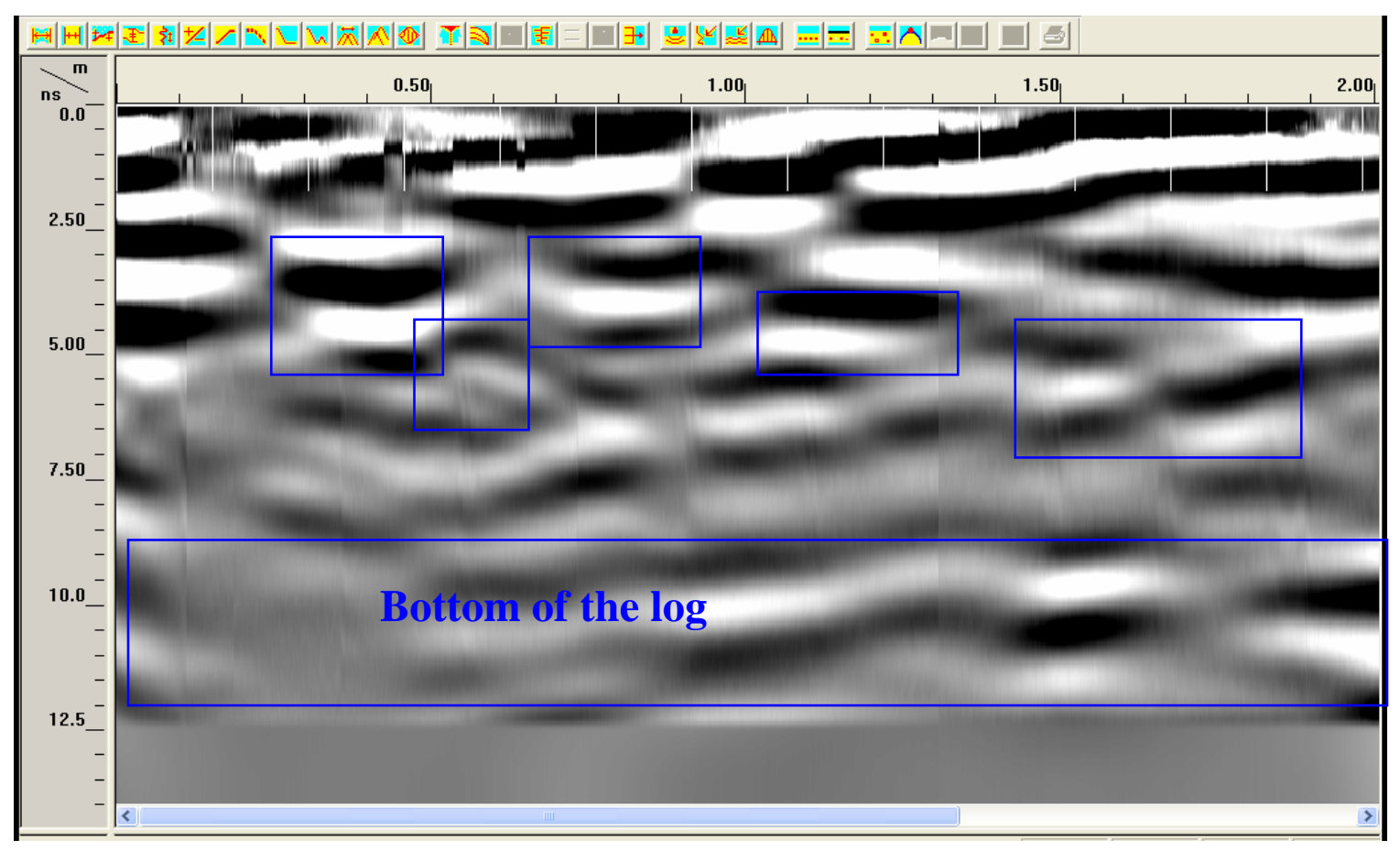

(a)

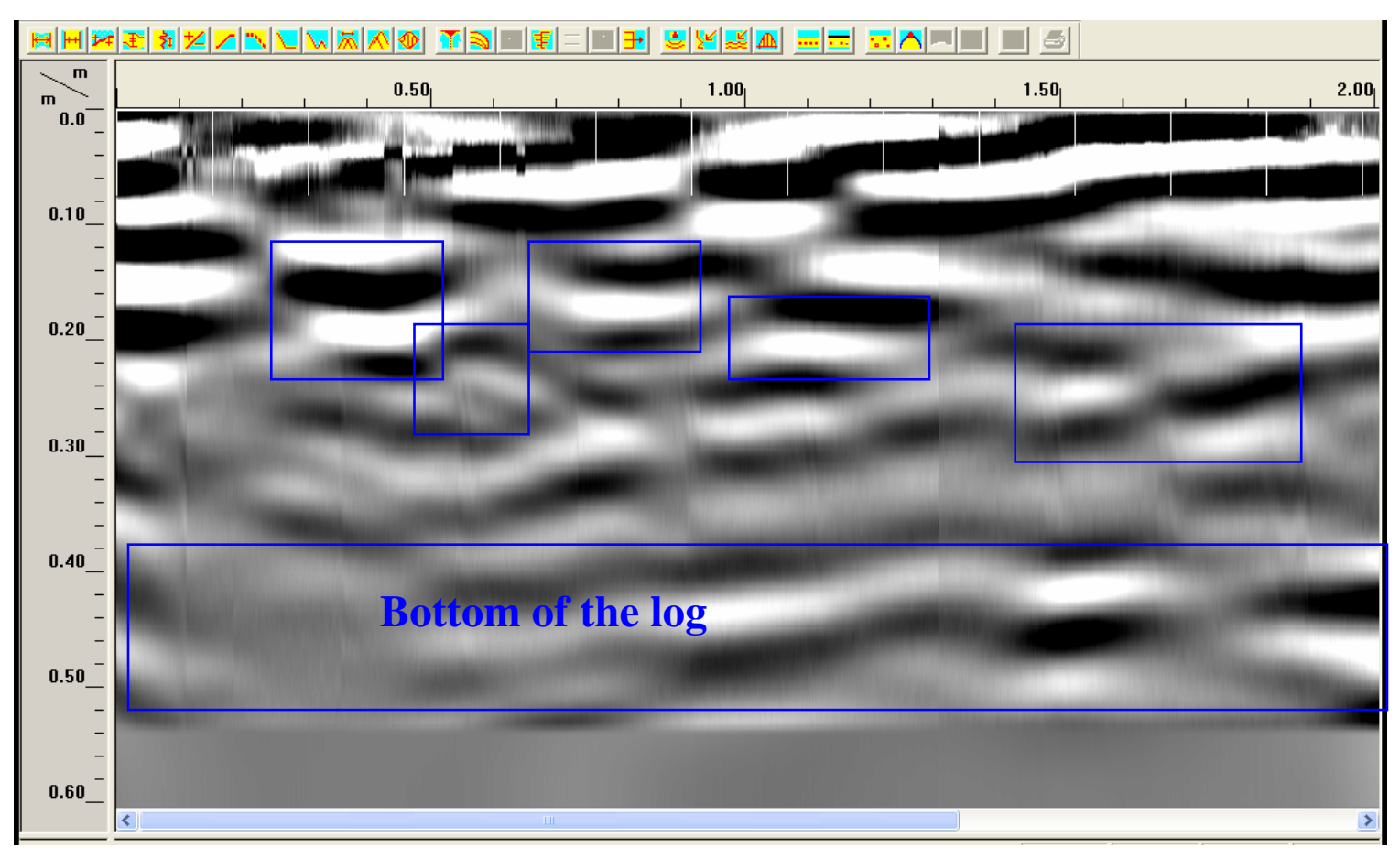

(b)

Figure 5-50 Processed GPR data of $\log 5$ (san \# 5), horizontal scale indicating the travel distance along the length of the $\log (\mathrm{m})$ and the vertical scale indicating (a) time (ns) (b) depth (m) 


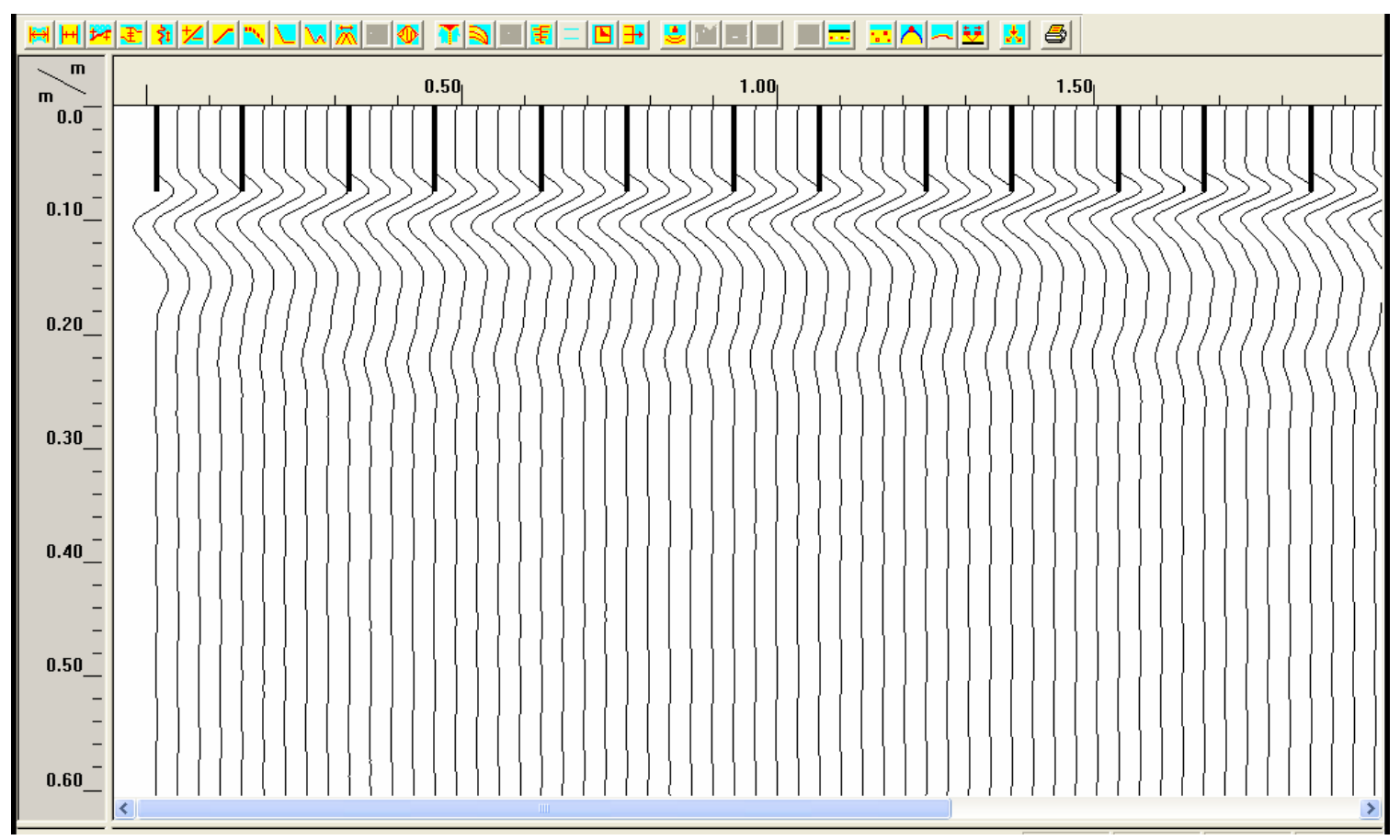

(a)

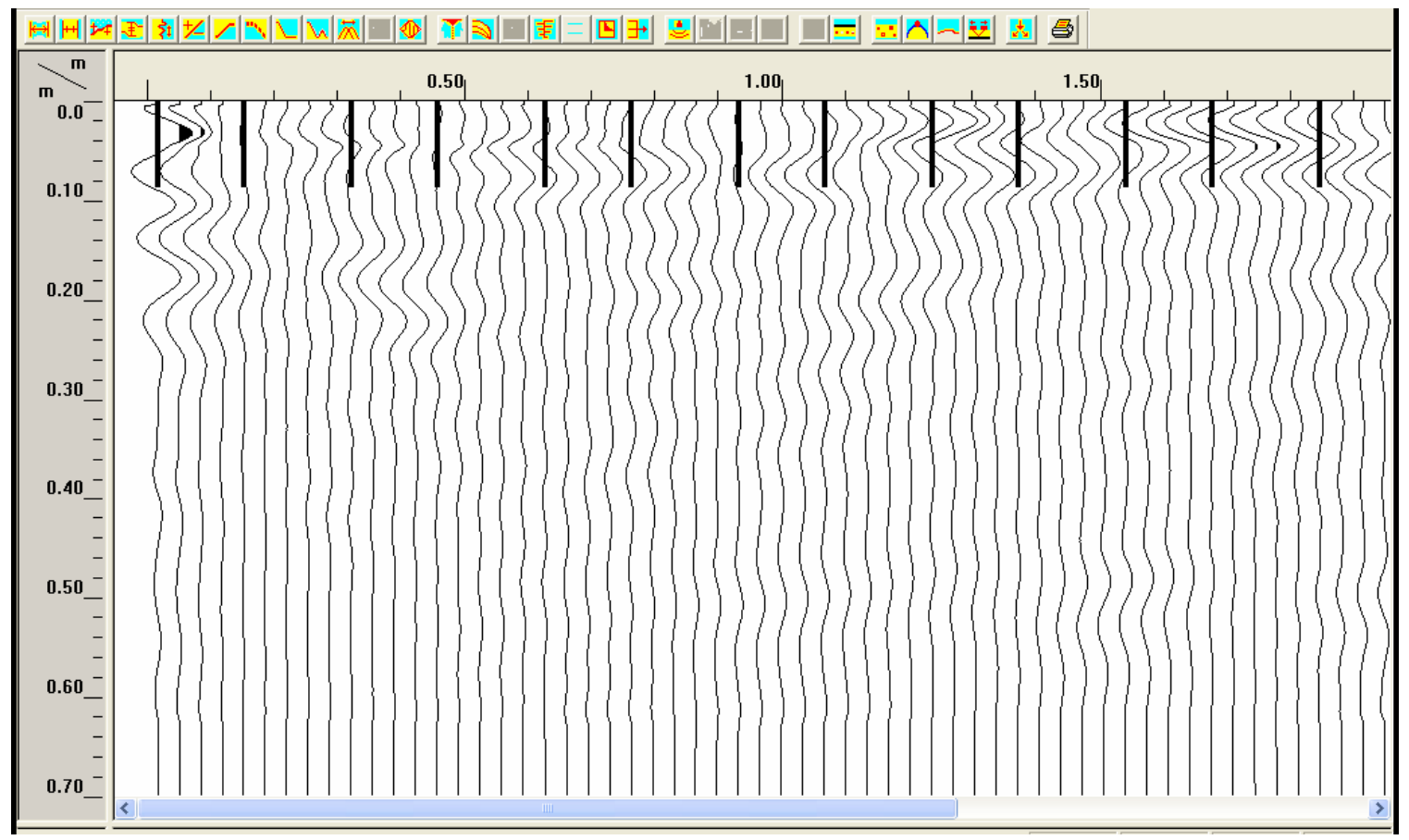

(b)

Figure 5-51 Wiggle plot of Log 5 (scan \# 5), horizontal scale indicating travel distance along the length of the $\log (\mathrm{m})$ and vertical scale indicating the depth $(\mathrm{m})$, for (a) raw data, and (b) processed data 


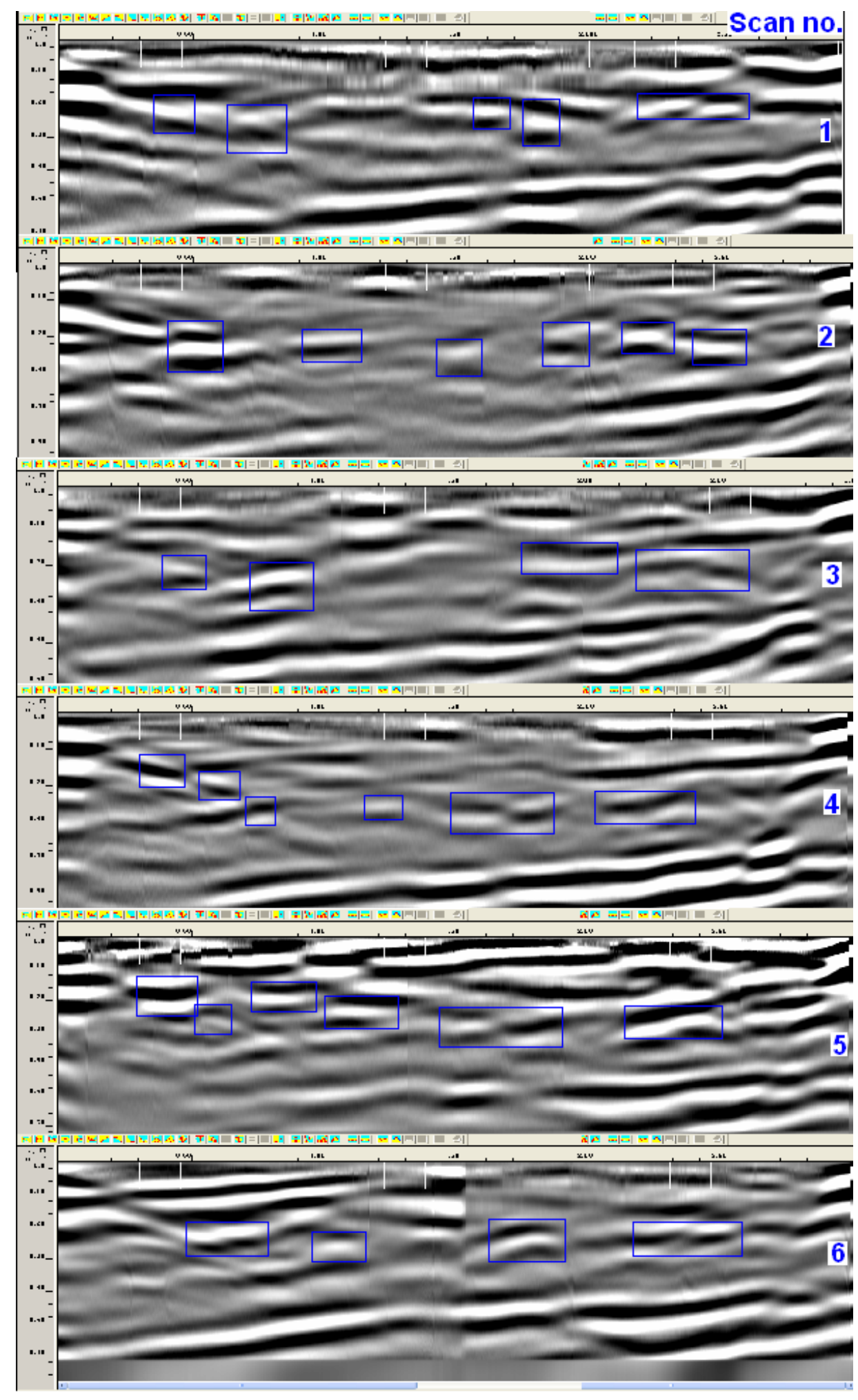

Figure 5-52 Processed data of Log 5 for all six scans, with the predicted internal features marked for mapping subsurface defects 


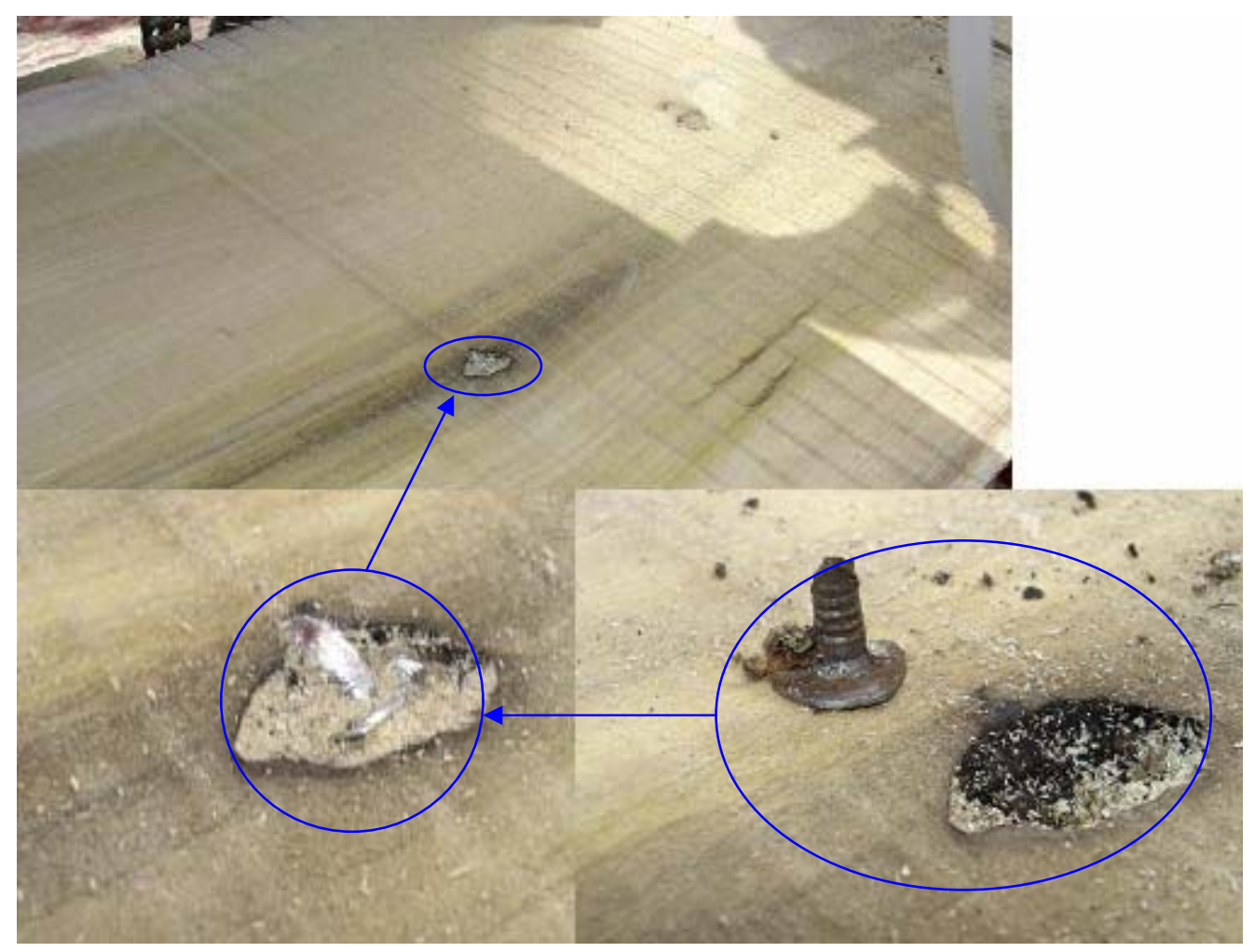

Figure 5-53 Metal defect

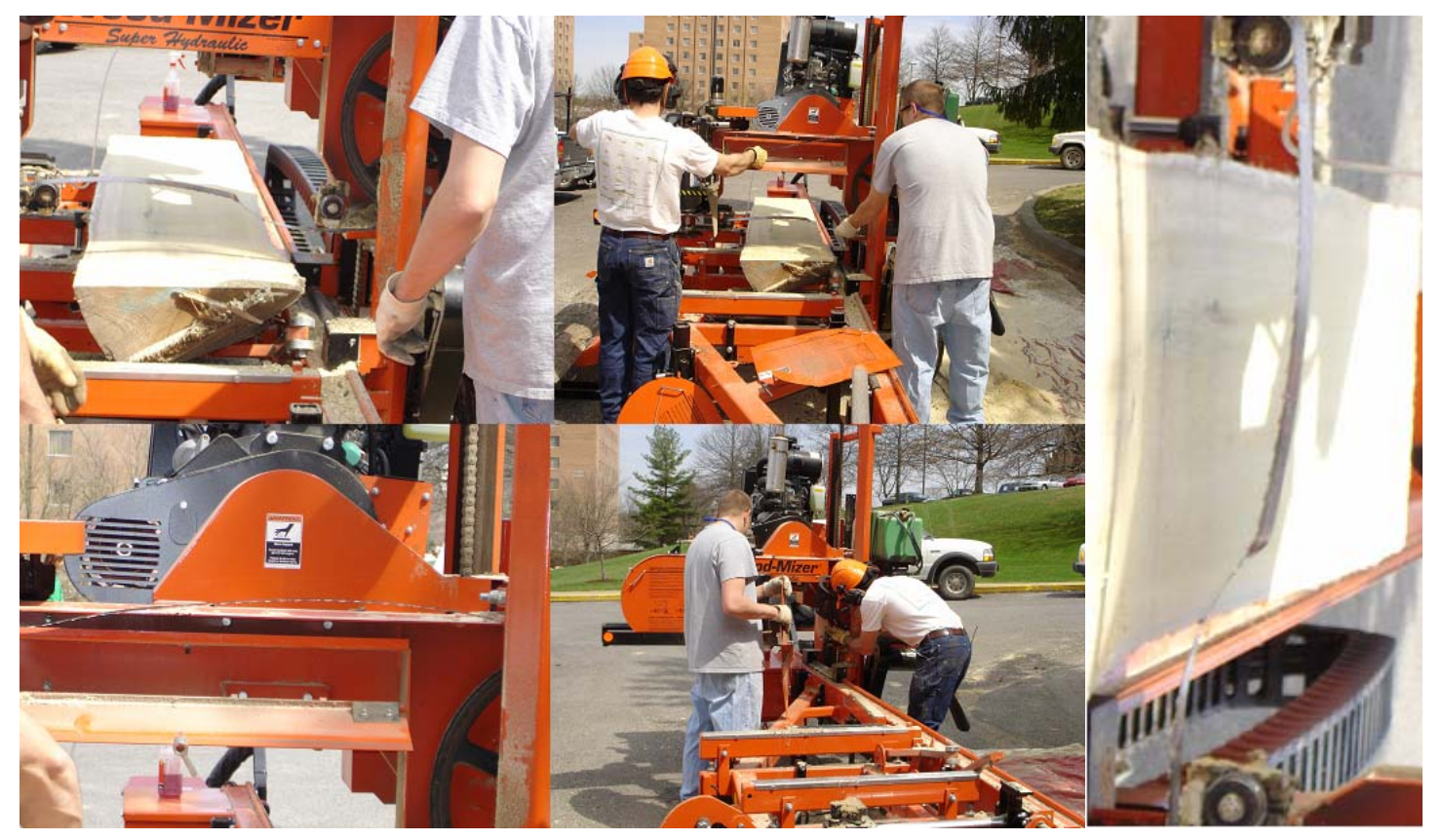

Figure 5-54 Picture showing the problems faced after the saw blade hit the small metal piece and got bent, and subsequent replacement of the blade 


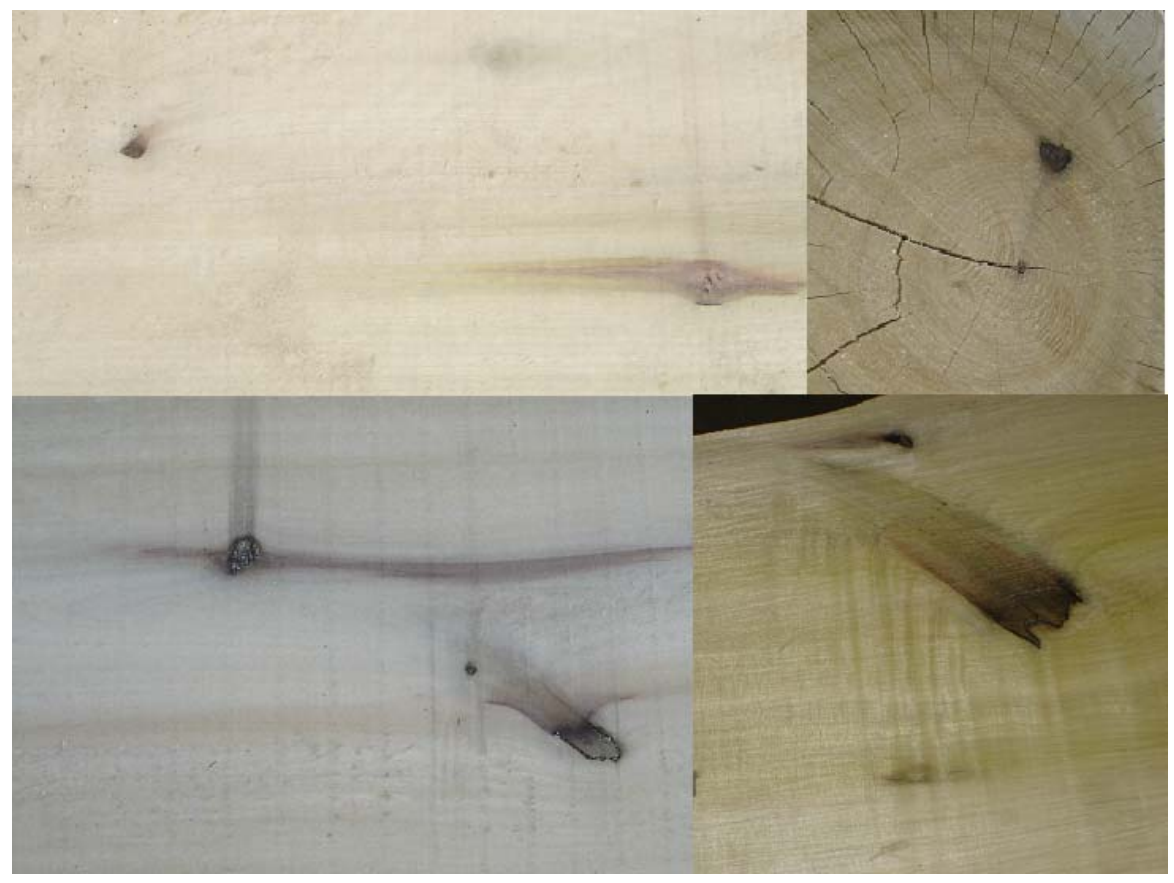

Figure 5-55 Knots in Log 5

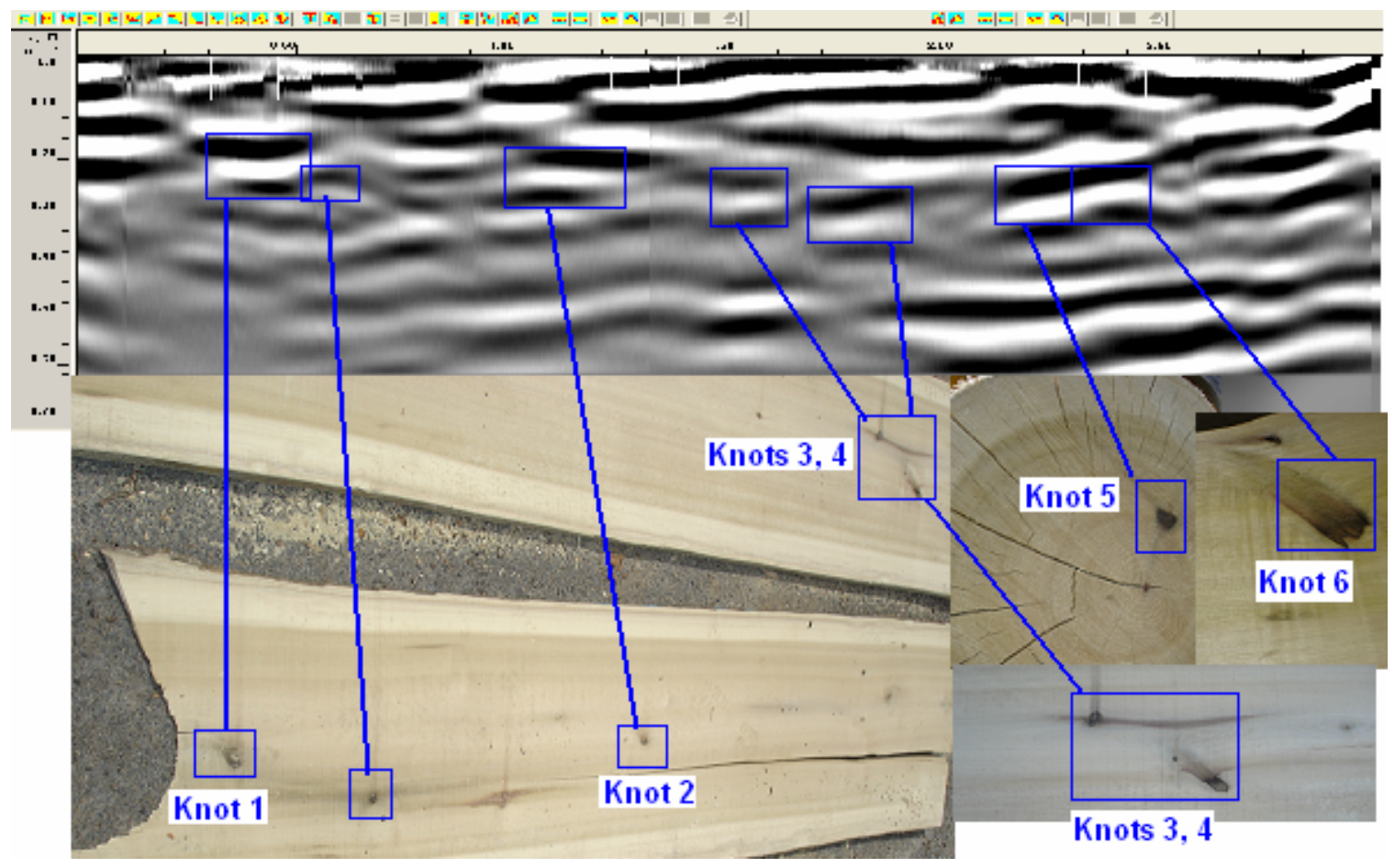

Figure 5-56 Correlation of processed data and defects after sawing the log 


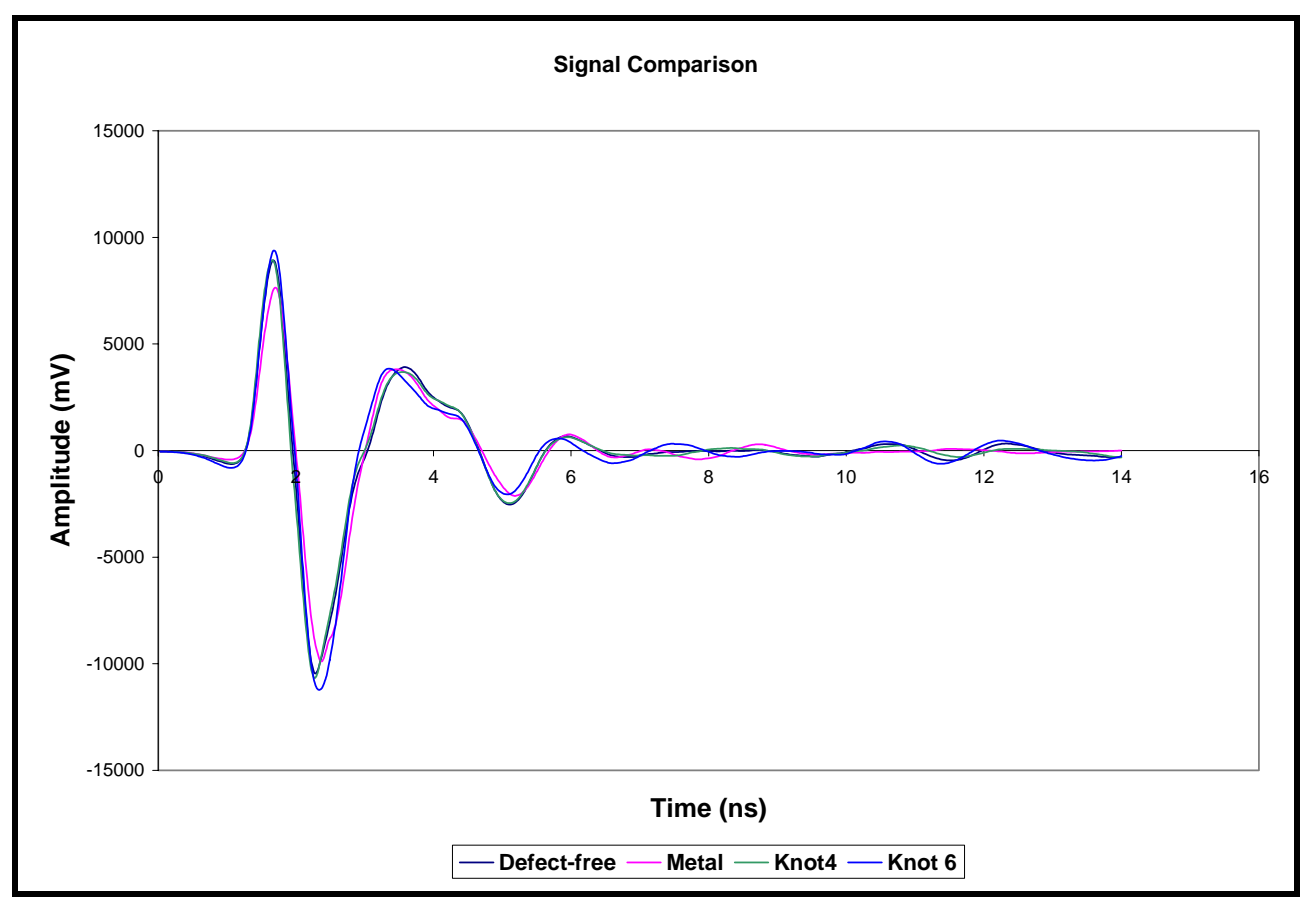

(a)

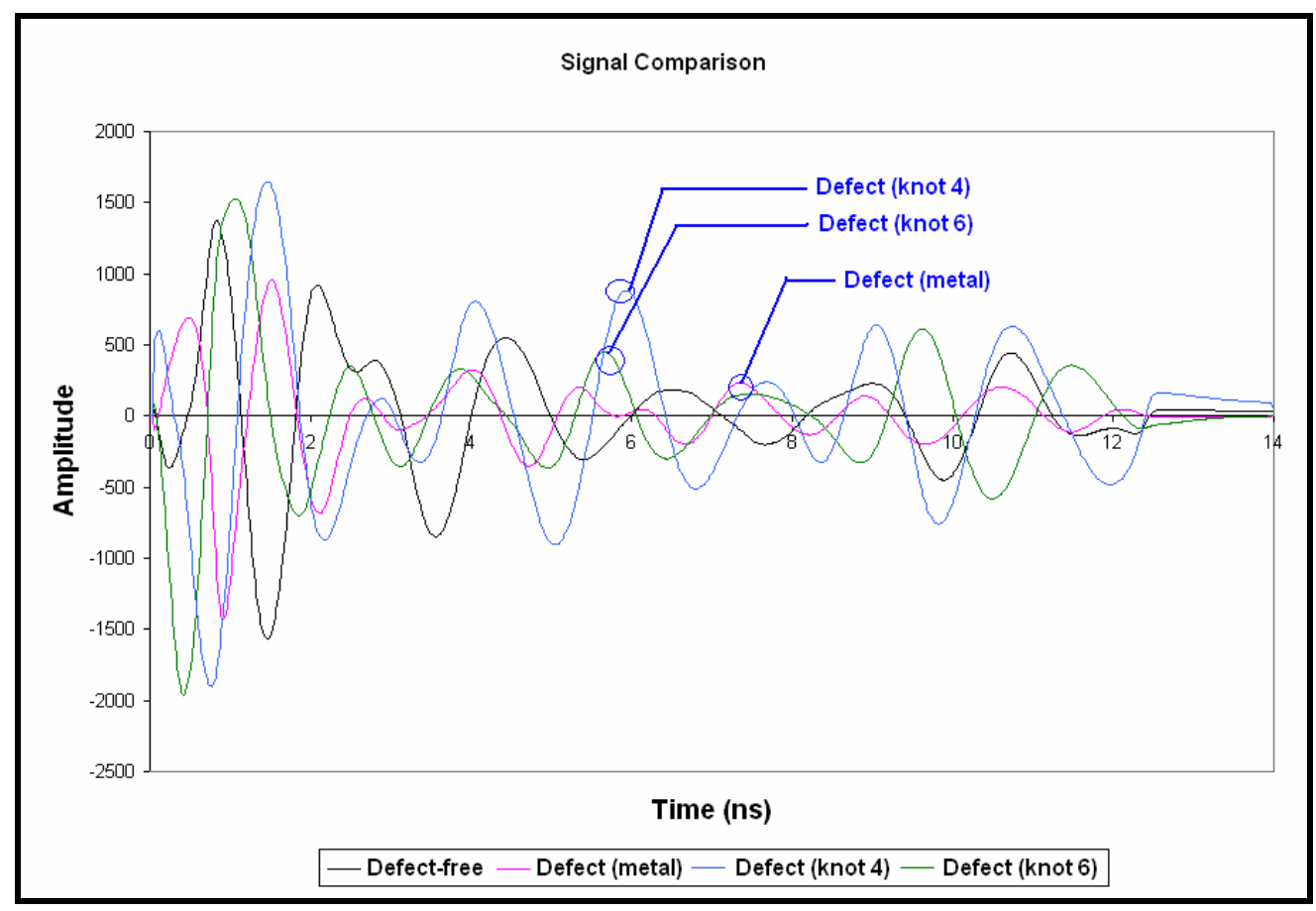

(b) 


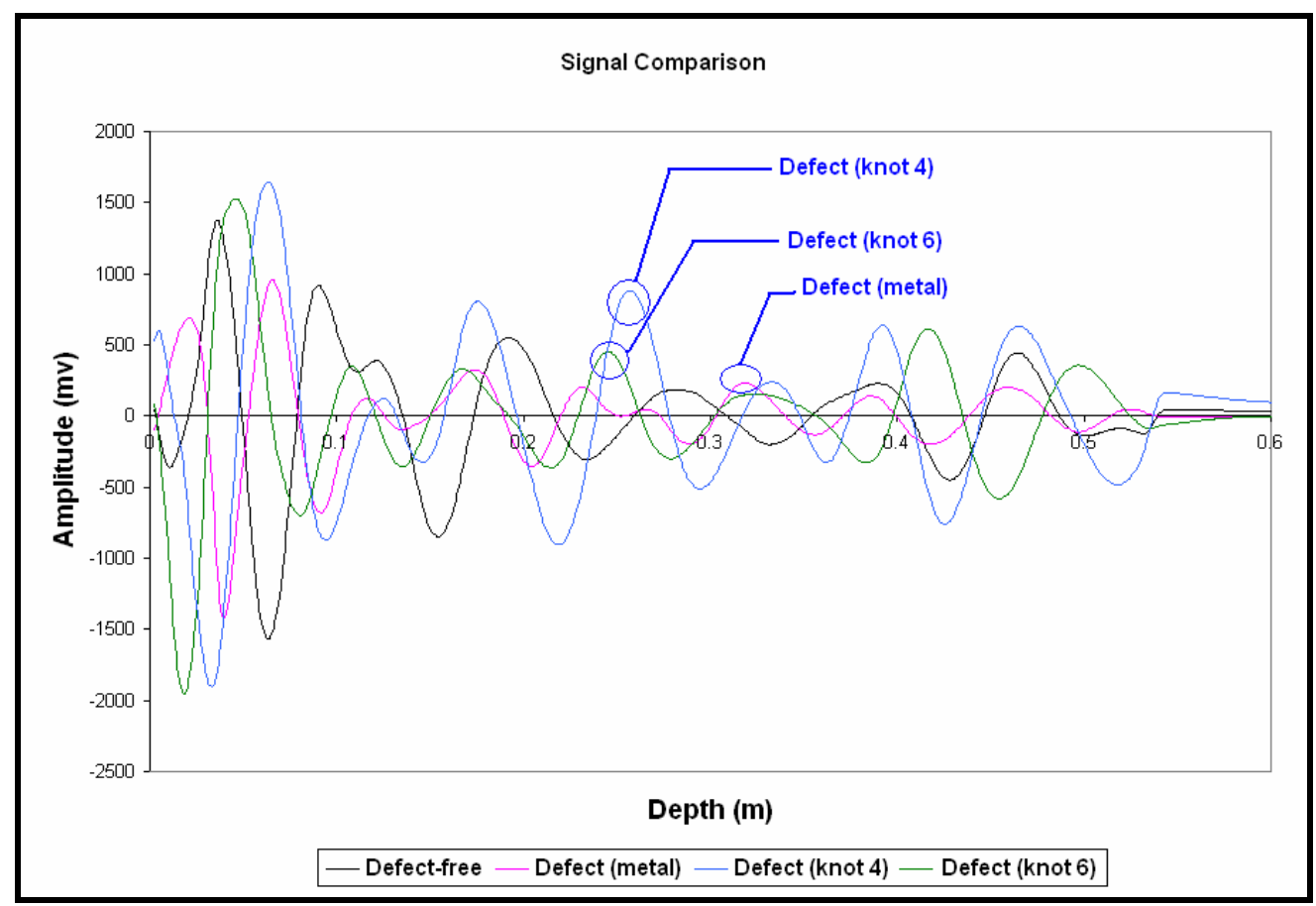

(c)

Figure 5-57 Comparison of GPR waveforms from sections with and without defects (knots and metal) in Log 5 for (a) raw data (Amplitude vs. Time), (b) processed data (Amplitude vs. Time), and (c) processed data (Amplitude vs. Depth) 


\section{$5.6 \quad$ LOG 6}

Table 5-9 Characteristics of Log 6

\begin{tabular}{|c|c|c|c|c|c|c|c|c|}
\hline Log & Species & $\begin{array}{c}\text { Length } \\
\text { no. }\end{array}$ & \multicolumn{2}{|c|}{ Diameter (m) } & \multicolumn{2}{|c|}{ Moisture Content (\%) } & Dielectric \\
& Butt & $\begin{array}{c}\text { Small } \\
\text { end }\end{array}$ & Surface & Max. & Average & Constant \\
\hline 6 & $\begin{array}{c}\text { Yellow } \\
\text { Poplar }\end{array}$ & 2.1 & 0.4 & 0.38 & 16 & 99 & 24.2 & 9 \\
\hline
\end{tabular}

The characteristics of Log 6 are given in Table 5-9. Figure 5-58 shows Log 6 and the markings made for scanning. The decay in the center of the log was very obvious. A single antenna, positioned above mark 1 as shown in Figure 5-59, was used for scanning the log. The data collection and processing parameters are shown in Figure 5-60. These parameters are explained in detail in Sections 4.1 and 4.2.

The process of data analysis for this $\log$ was similar to that for Log 1. Figure 5-61 shows raw GPR scan for $\log 6$. The top set of white-black-white bands indicates the transmit signal. Another feature at a distance of 0.1 to $0.3 \mathrm{~m}$ and at $4 \mathrm{~ns}(0.2 \mathrm{~m})$ depth was observed in the raw data. It was very difficult to predict any other internal features from the raw GPR scans in Figure 5-61.

The extent of decay and other internal defects was predicted based on the postprocessed GPR data shown in Figure 5-62. Signal clutter extending from the butt end to a distance of $0.4 \mathrm{~m}$ at a depth of $0.15 \mathrm{~m}$ was observed. This high amplitude signal clutter was due to heavy decay observed at the butt end. Also, in Figure 5-62 there is an echo from the center core of the $\log$ (around $3.5 \mathrm{~ns}$ or $0.17 \mathrm{~m}$ ) staring from the butt end extending all the way to the small end. This was predicted to be due to rot initiation. Another internal feature (signal clutter) at a distance of $1.1 \mathrm{~m}$ along the length of the log and at a depth of $0.2 \mathrm{~m}$ was predicted (Figure 5-62(b)). The reflection from the bottom of the log was visible at $6.5 \mathrm{~ns}(0.28 \mathrm{~m})$ at the starting point of the scan and its depth of occurrence varied along the length of the log with a bulge occurring between horizontal distance of $1 \mathrm{~m}$ and $1.5 \mathrm{~m}$. The variable depth of the bottom reflection as the antenna 
traveled along the surface of the log, with higher depth in the center, could have been due to high moisture content in the central region.

The wiggle plots shown in Figure 5-63 were confusing and not of much use in predicting the internal features in the log.

To validate the GPR predictions, the log was sawed into circular cross sections using an 18 inch $(0.38 \mathrm{~m})$ chain saw at 4 different locations. These cross sections are shown in Figure 5-64. The same cross section pictures and the processed GPR data in Figure 5-62(b) were combined in Figure 5-65 to show the correlation between the predicted and actual internal features. The actual region of heavy decay extended from but end to a distance of $0.4 \mathrm{~m}$ at a depth of $0.15 \mathrm{~m}$, which was also accurately predicted from the processed GPR data. The rot initiation (small amount of decay) was prominent in the center core of the log and was found to be extending from the butt end to the small end, which matched the GPR predictions very well. The signal clutter observed near the $1.1 \mathrm{~m}$ mark in the processed GPR data was found to be a region of high (99\%) moisture content as can be seen in Figure 5-65 and 5-64(c). This high moisture region also resulted in increased dielectric constant and signal travel time, causing a bulge in the GPR reflection from the bottom of the $\log$ as seen in Figures 5-65 and 5-62.

Overall there was an excellent correlation between the location of defects predicted by GPR and those found after sawing the log. 


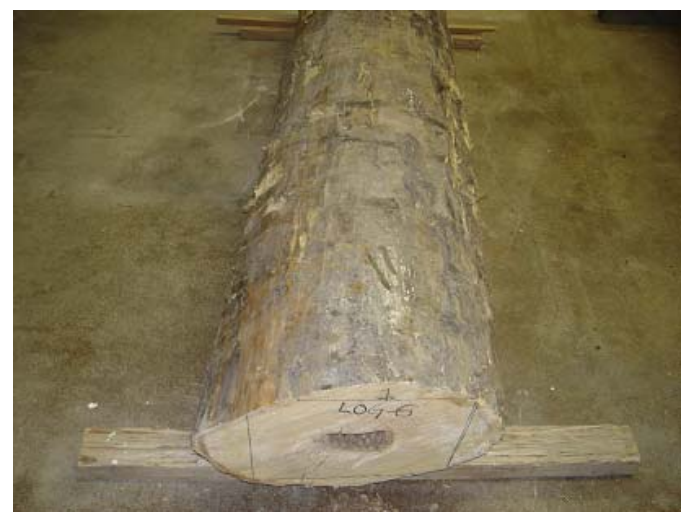

(a)

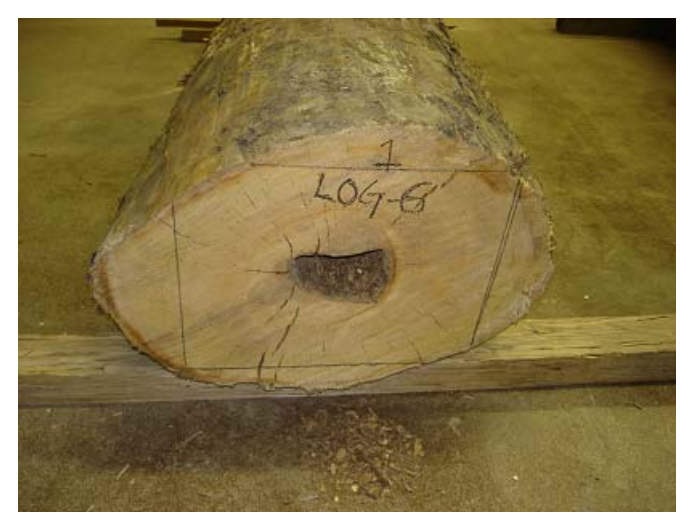

(b)

Figure 5-58 Picture of (a) $\log 6$, and (b) rot (cavity) seen clearly at the butt end

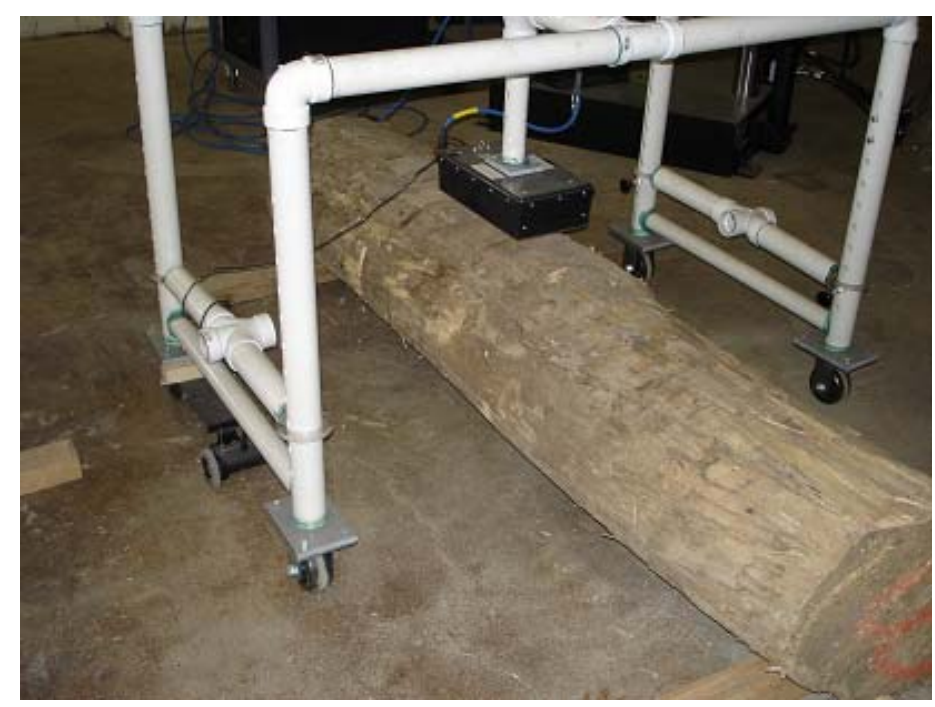

Figure 5-59 Antenna configuration for $\log 6$ 


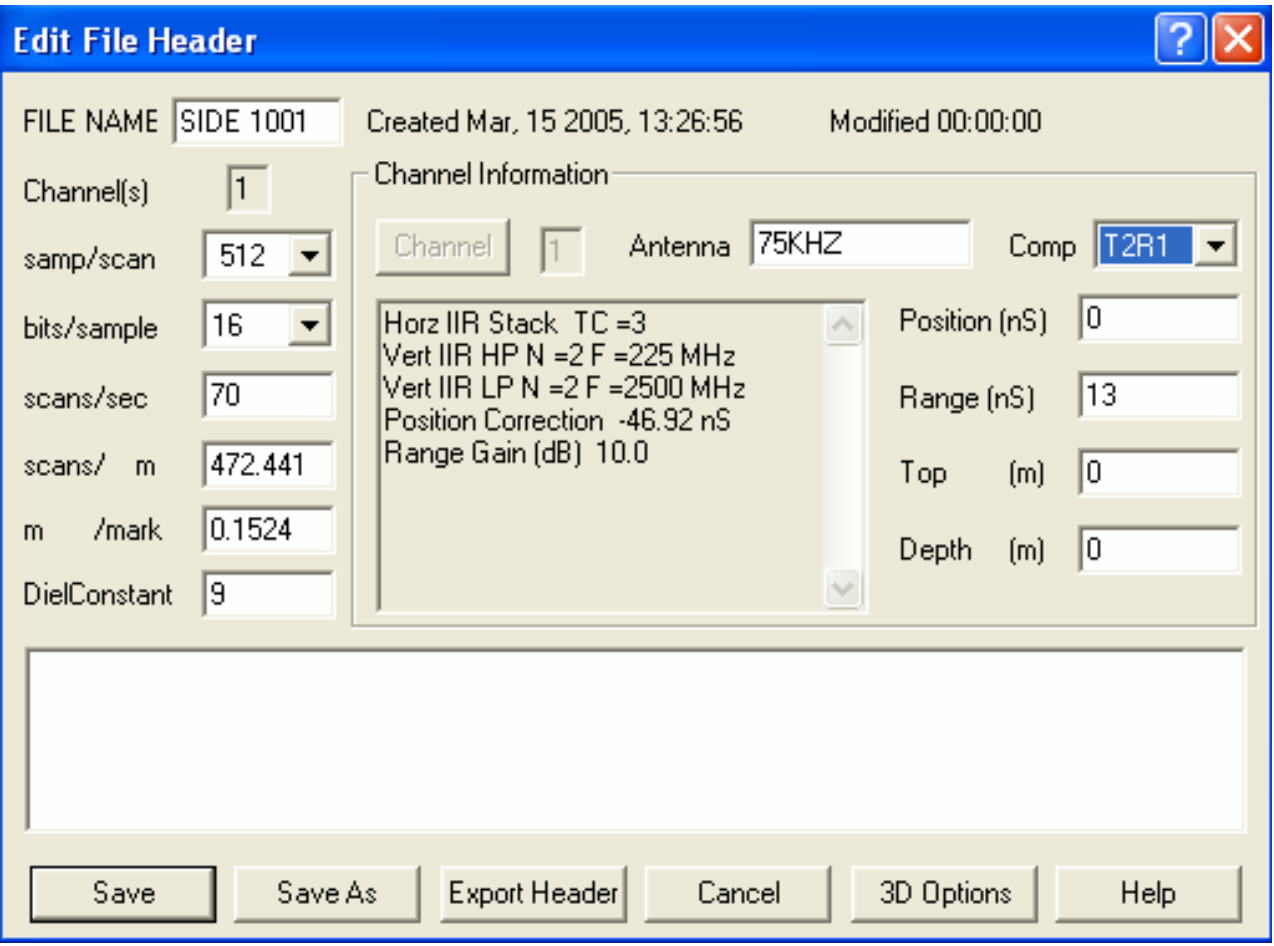

(a)

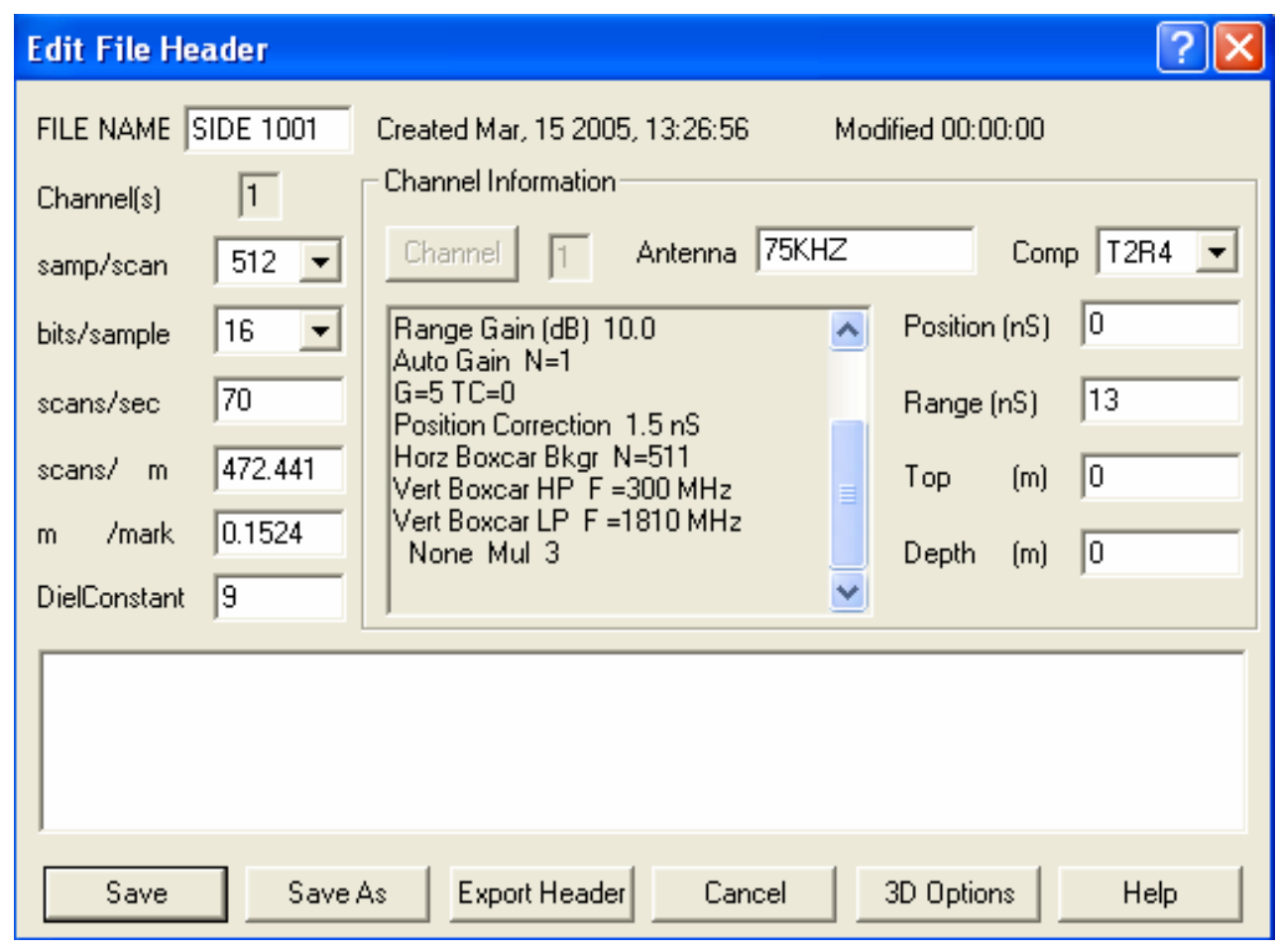

(b)

Figure 5-60 (a) Data collection parameters (b) processing parameters 


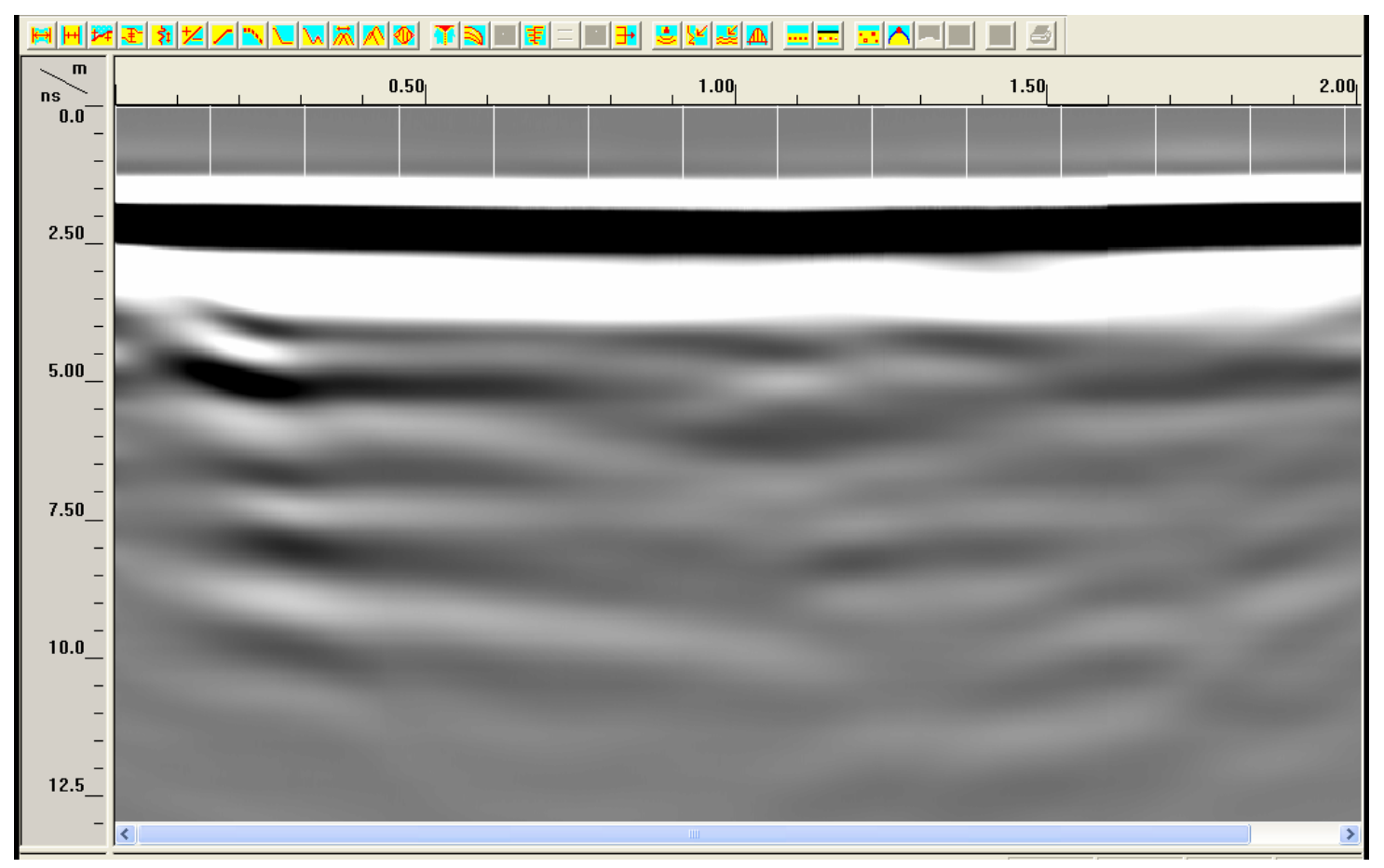

(a)

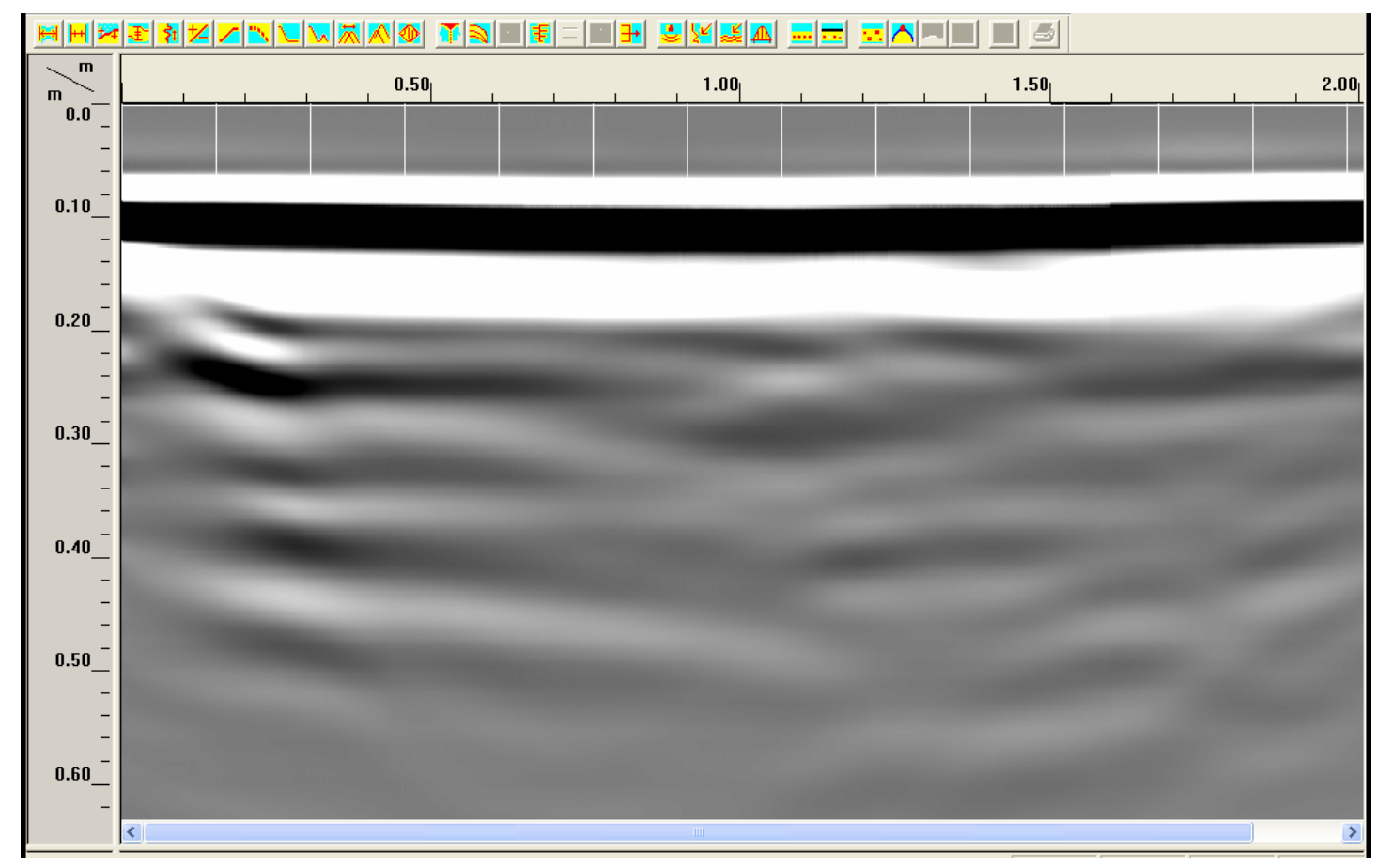

(b)

Figure 5-61 Raw GPR data of $\log$ 6, horizontal scale indicating the travel distance along the length of the $\log (\mathrm{m})$ and the vertical scale indicating (a) time (ns) (b) depth (m) 


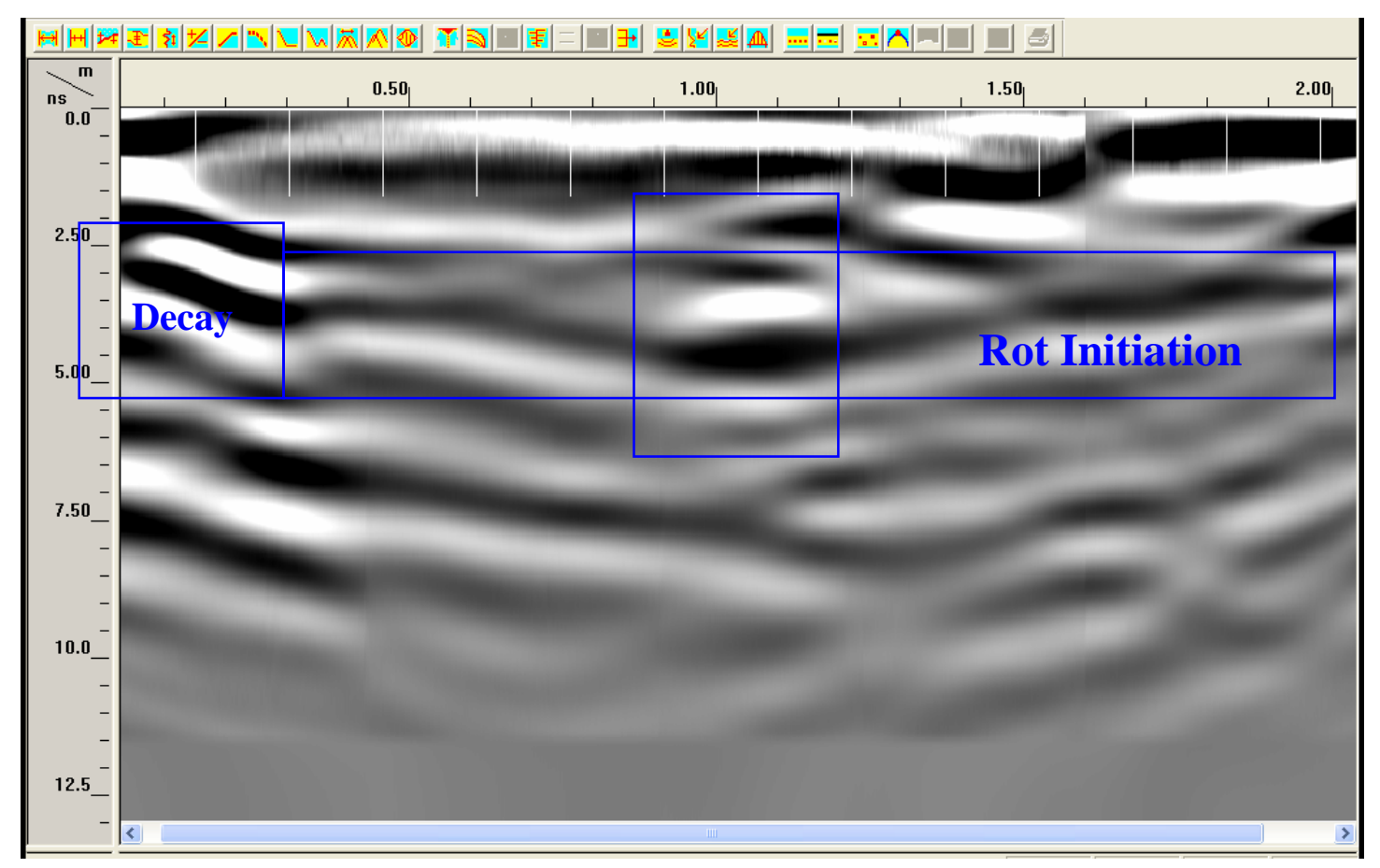

(a)

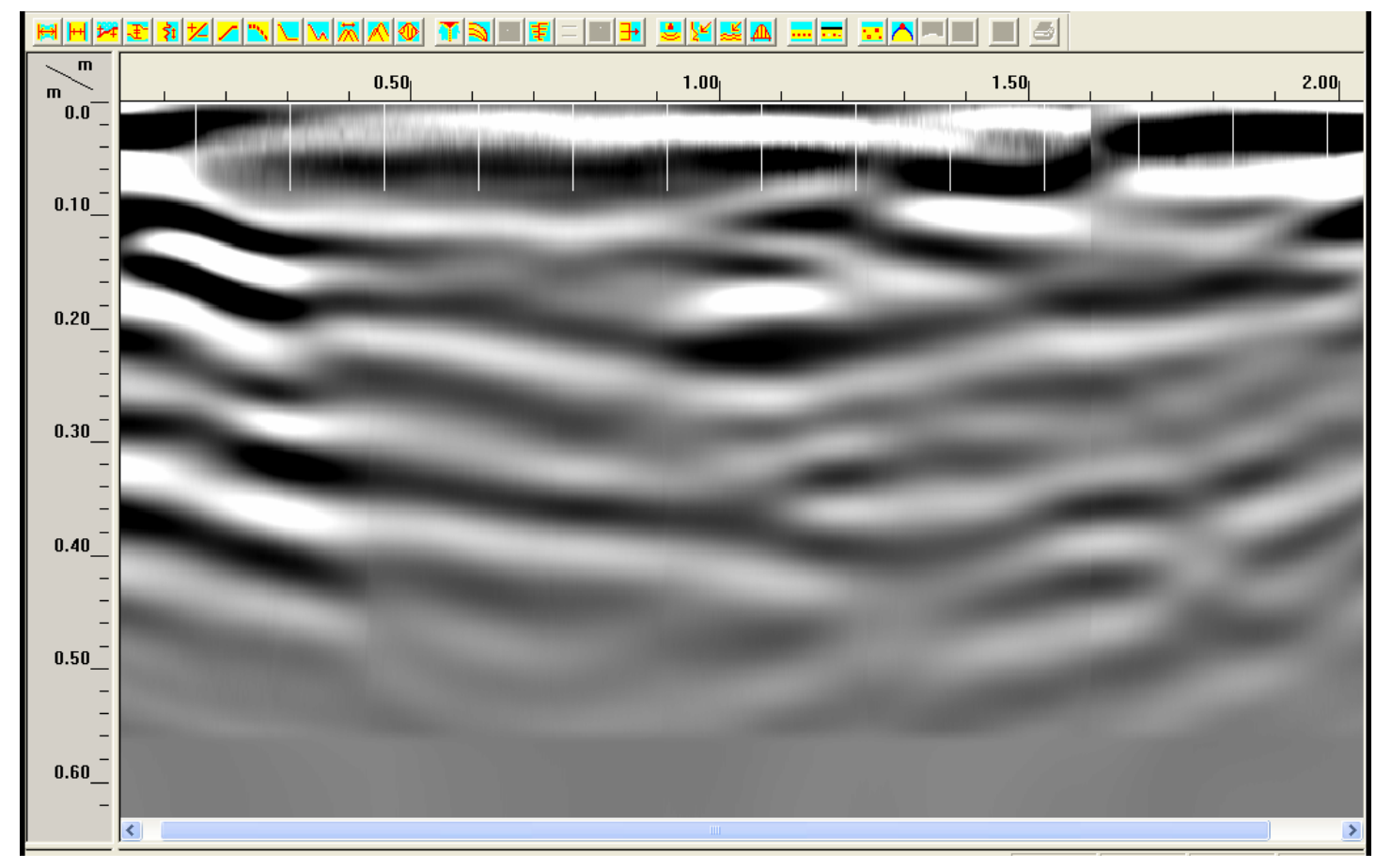

(b)

Figure 5-62 Processed GPR data of $\log$ 6, horizontal scale indicating the travel distance along the length of the $\log (\mathrm{m})$ and the vertical scale indicating (a) time (ns) (b) depth (m) 


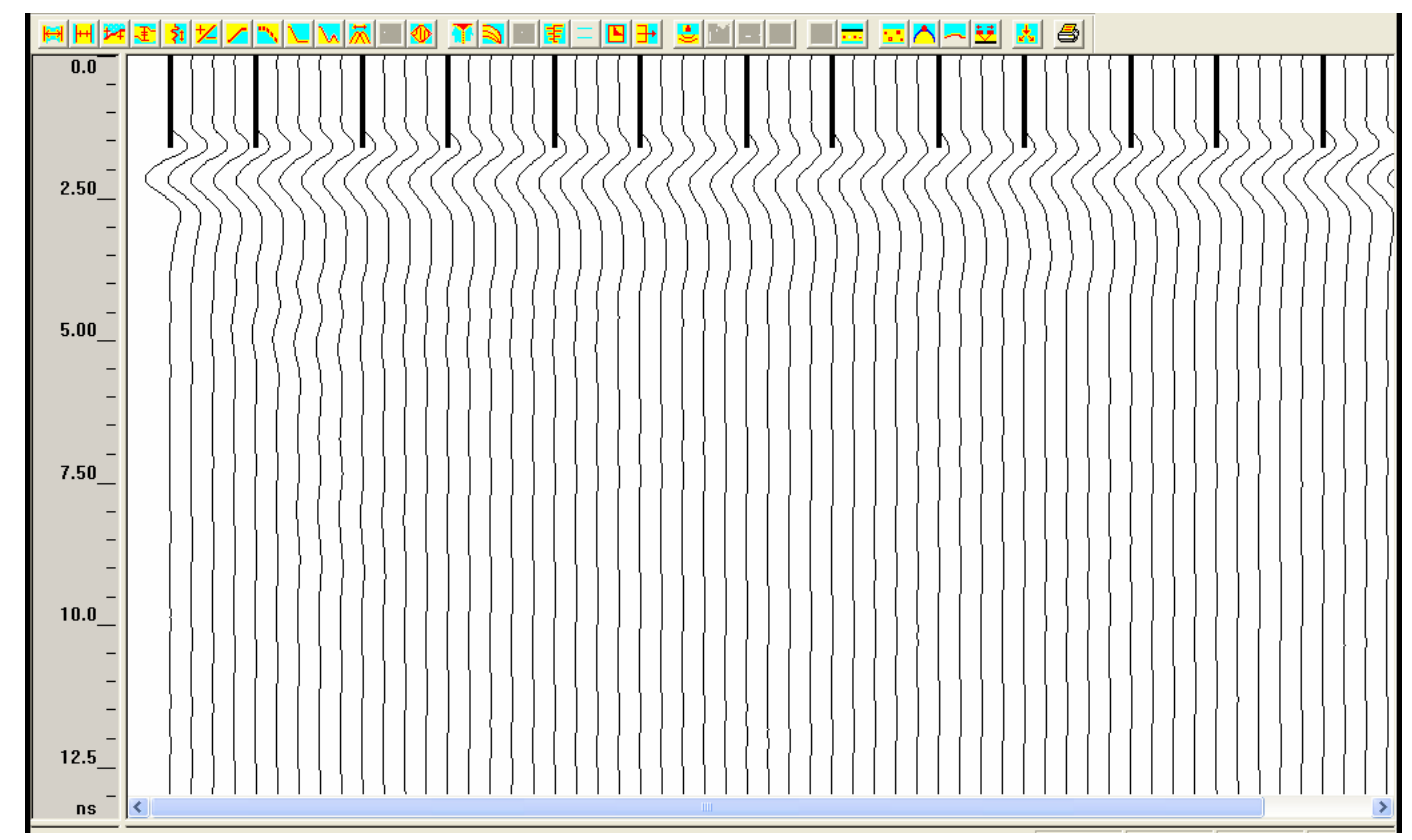

(a)

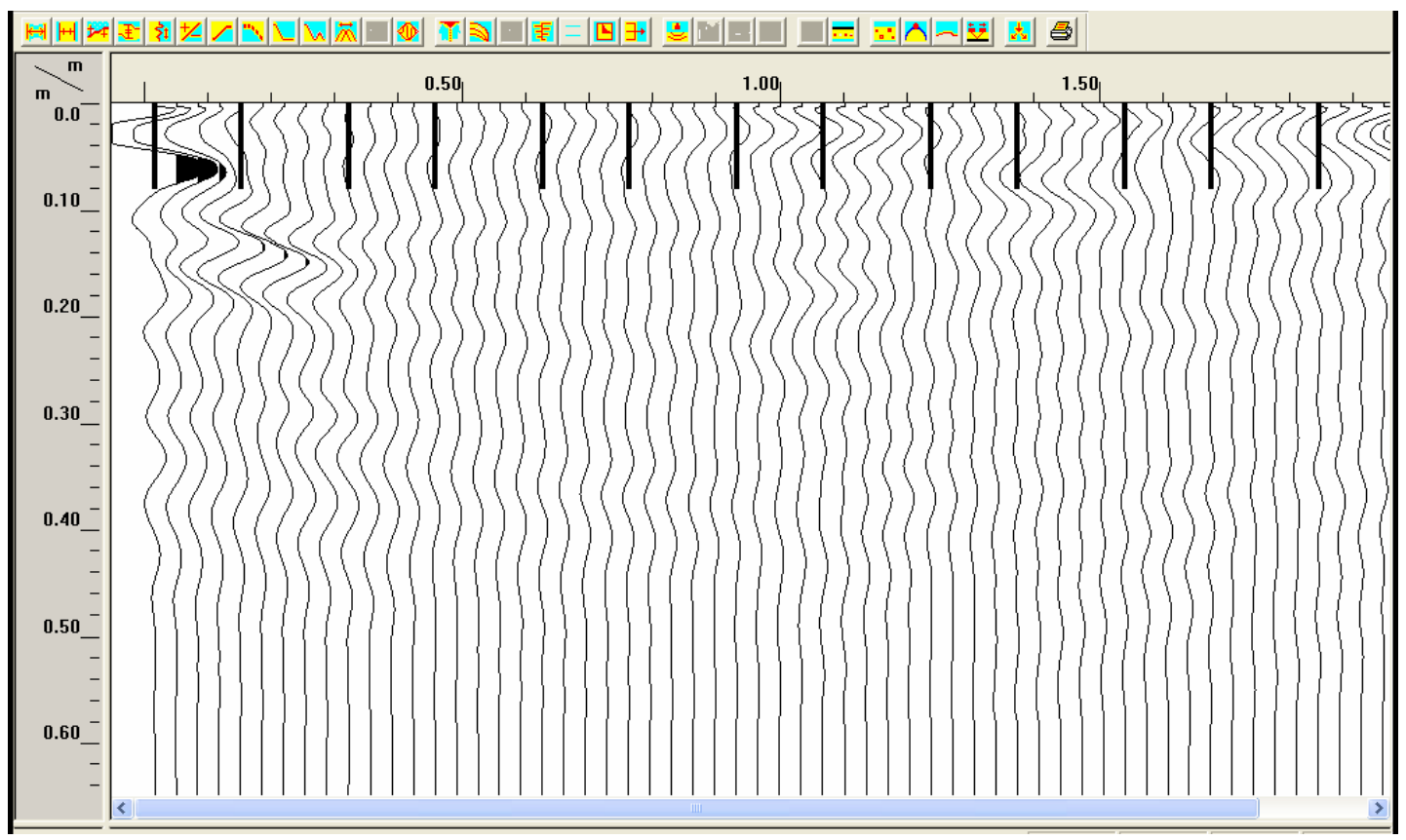

(b)

Figure 5-63 Wiggle plot of Log 6, horizontal scale indicating travel distance along the length of the $\log (\mathrm{m})$ and vertical scale indicating the depth $(\mathrm{m})$, for (a) raw data, and (b) processed data 


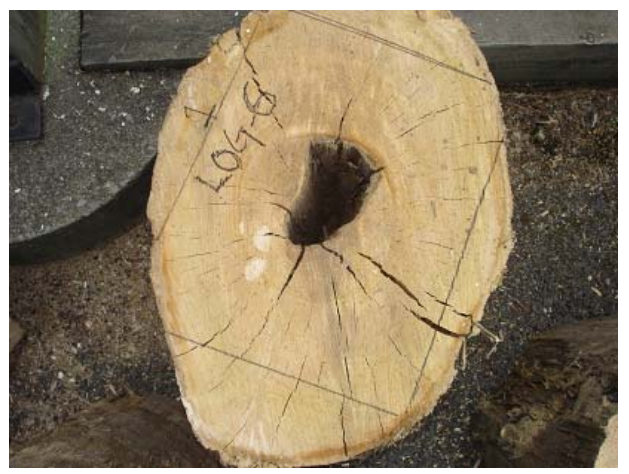

(a)

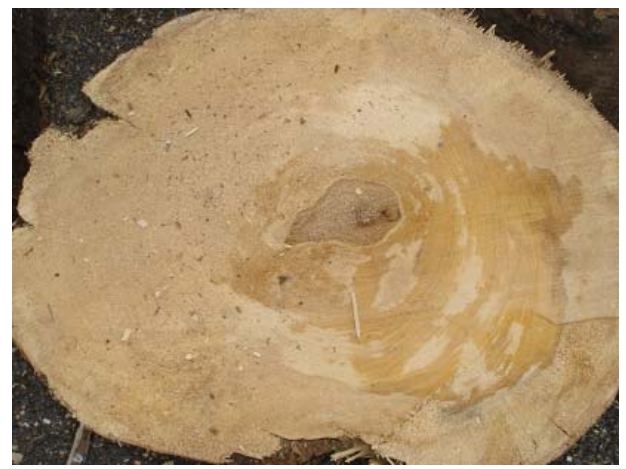

(c)

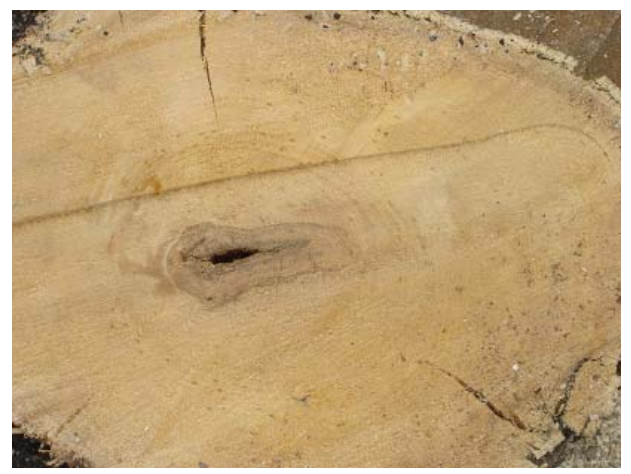

(b)

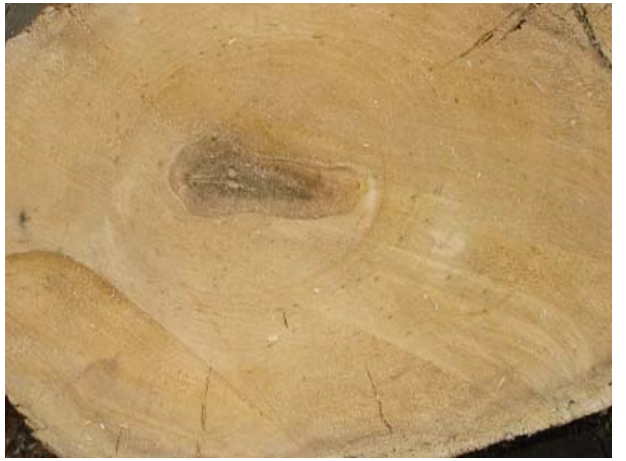

(d)

Figure 5-64 Picture showing cut sections of Log 4

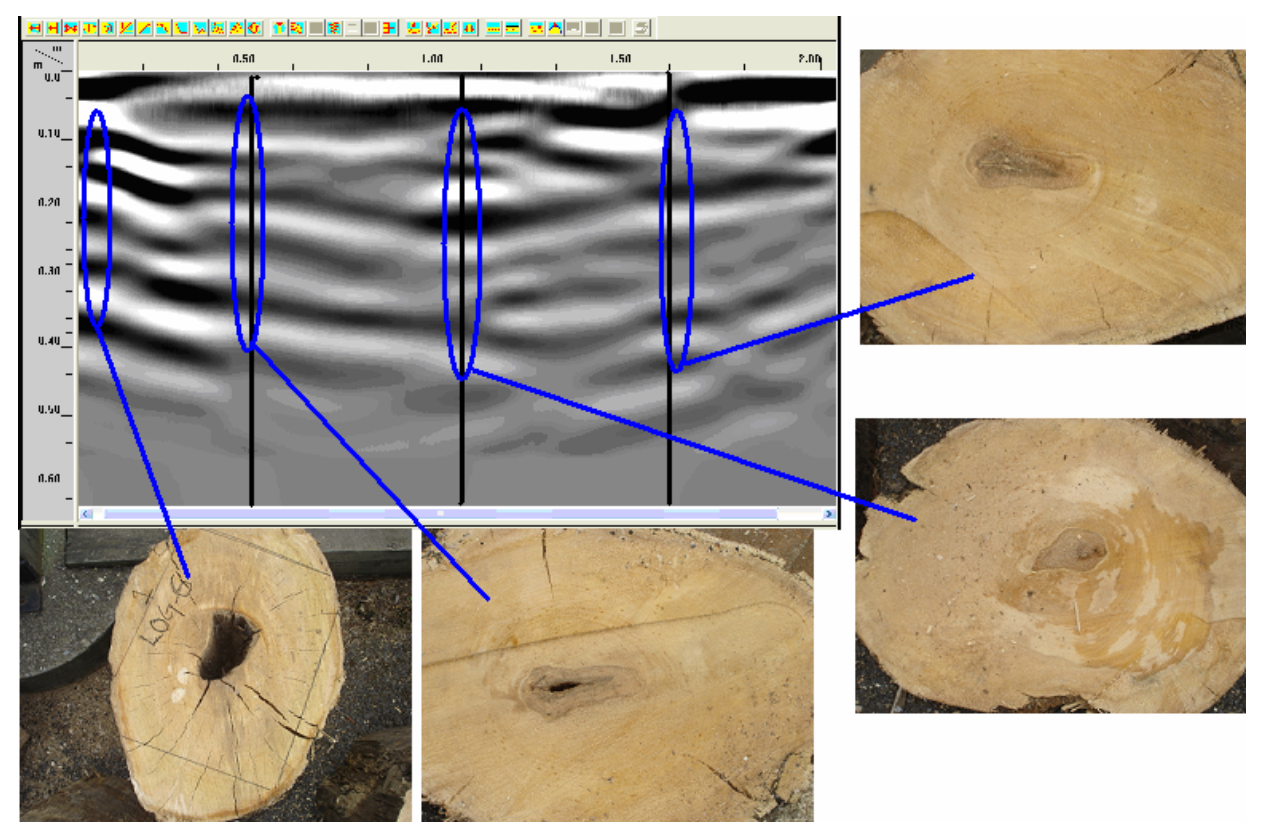

Figure 5-65 Processed GPR data of Log 6 and corresponding cut sections showing internal features 


\section{$5.7 \quad$ LOG 7}

Table 5-10 Characteristics of $\log 7$

\begin{tabular}{|c|c|c|c|c|c|c|c|c|}
\hline Log & \multirow{2}{*}{$\begin{array}{c}\text { Species } \\
\text { no. }\end{array}$} & $\begin{array}{c}\text { Length } \\
\mathbf{( m )}\end{array}$ & $\begin{array}{c}\text { Diameter (m) } \\
\text { Butt }\end{array}$ & $\begin{array}{c}\text { Small } \\
\text { end }\end{array}$ & Soisture Content (\%) & Dielectric \\
\hline 7 & Unknown & 2.31 & 0.36 & 0.34 & - & - & - & 12 \\
\hline
\end{tabular}

The available characteristics for the $\log 7$ are given in Table 5-10. The species and the moisture content of $\log 7$ were unknown. Though numbered 7, this was the first $\log$ tested to predict whether GPR can detect metals in logs or not. For this purpose two metal pieces; a metal bolt of 5/8 in $(0.016 \mathrm{~m})$ diameter and $0.203 \mathrm{~m}$ length and a metal rod of 6/8 in $(0.019 \mathrm{~m})$ diameter and $0.2 \mathrm{~m}$ length were inserted into the log as shown in Figure 5-66.

This $\log$ was scanned in free mode with the scans/sec and the antenna transmit frequency $(\mathrm{kHz})$ selected as 100 . The raw data was very long; hence stacking was done to compress the data. The free mode data file shown in Figure 5-67 is one-third the size of the original file. The processed data for $\log 7$ is given in Figure 5-68. The GPR scans in Figures 5-67 as well as 5-68 showed the embedded metal defects very well as distinct parabolic shaped signal clutters. However, these defects are more clearly visible as sharper parabolas in the processed GPR scan (Figure 5-68) since the processing removed the surface coupling reflection.

While the above figures showed the metal defects very well, it was not possible to predict their exact location in the free mode which does not use survey wheel for exact distance marking. Therefore, the other logs in this study (reported in the previous sections) were tested using the optical encoder based survey wheel. 


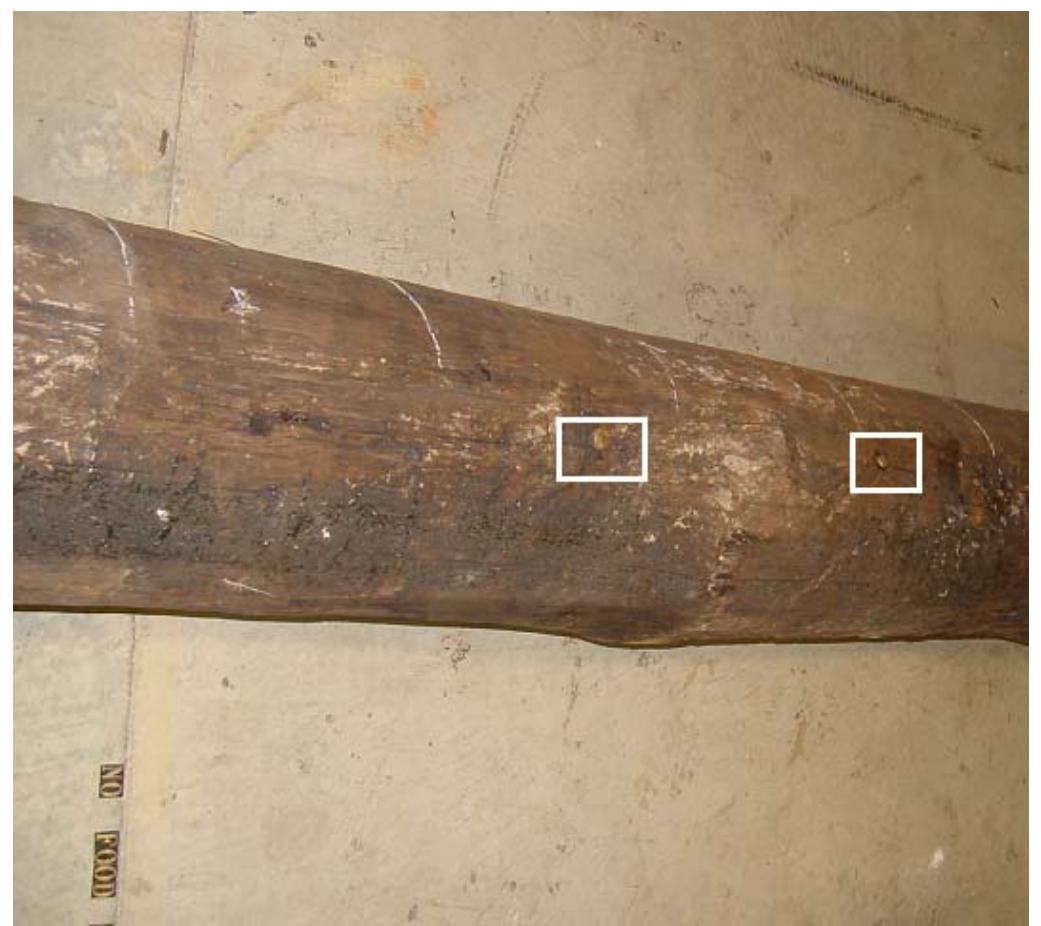

(a)

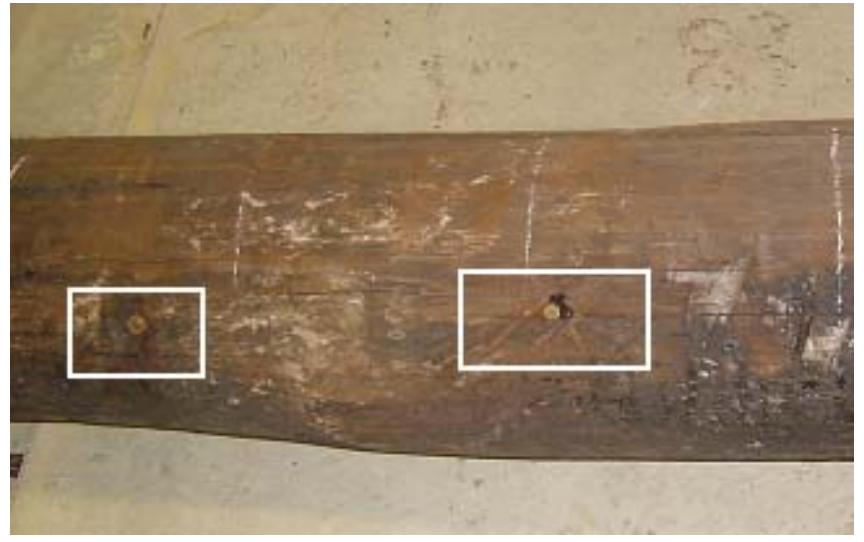

(b)

Figure 5-66 Picture of Log 7 showing inserted metal 


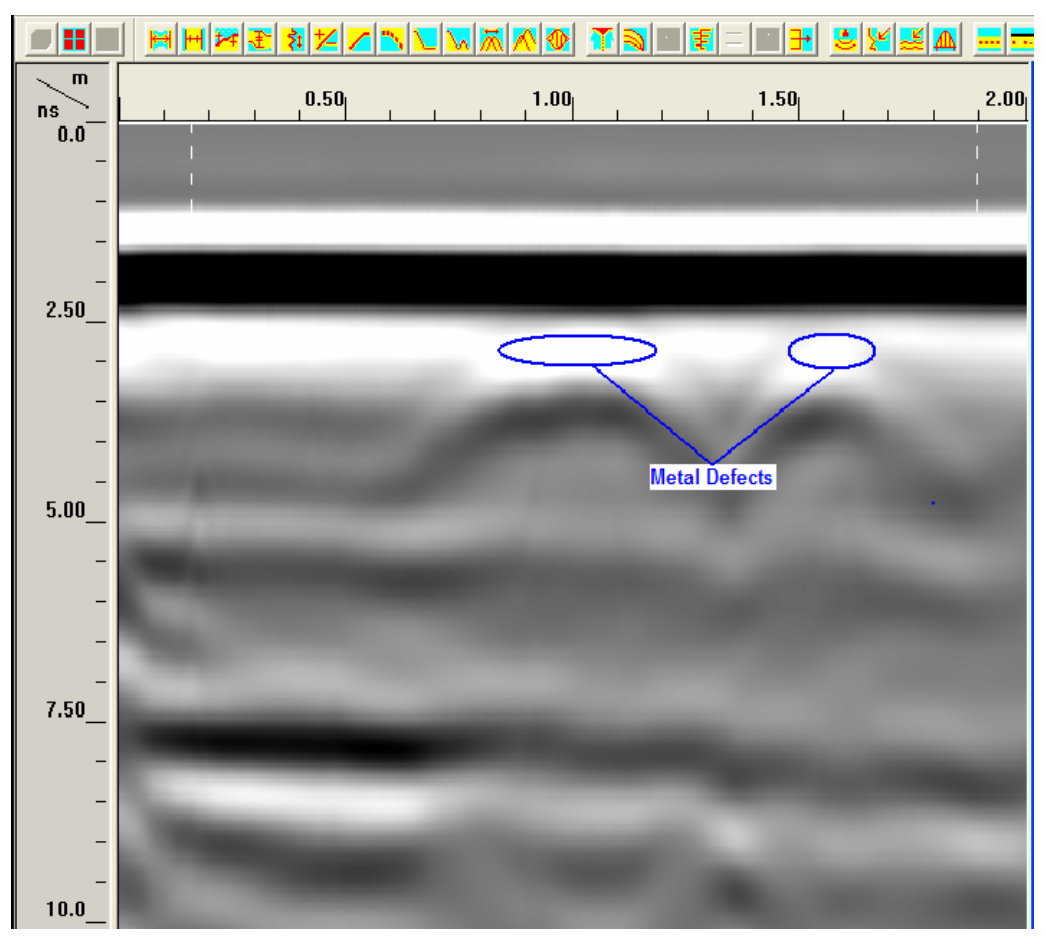

(a)

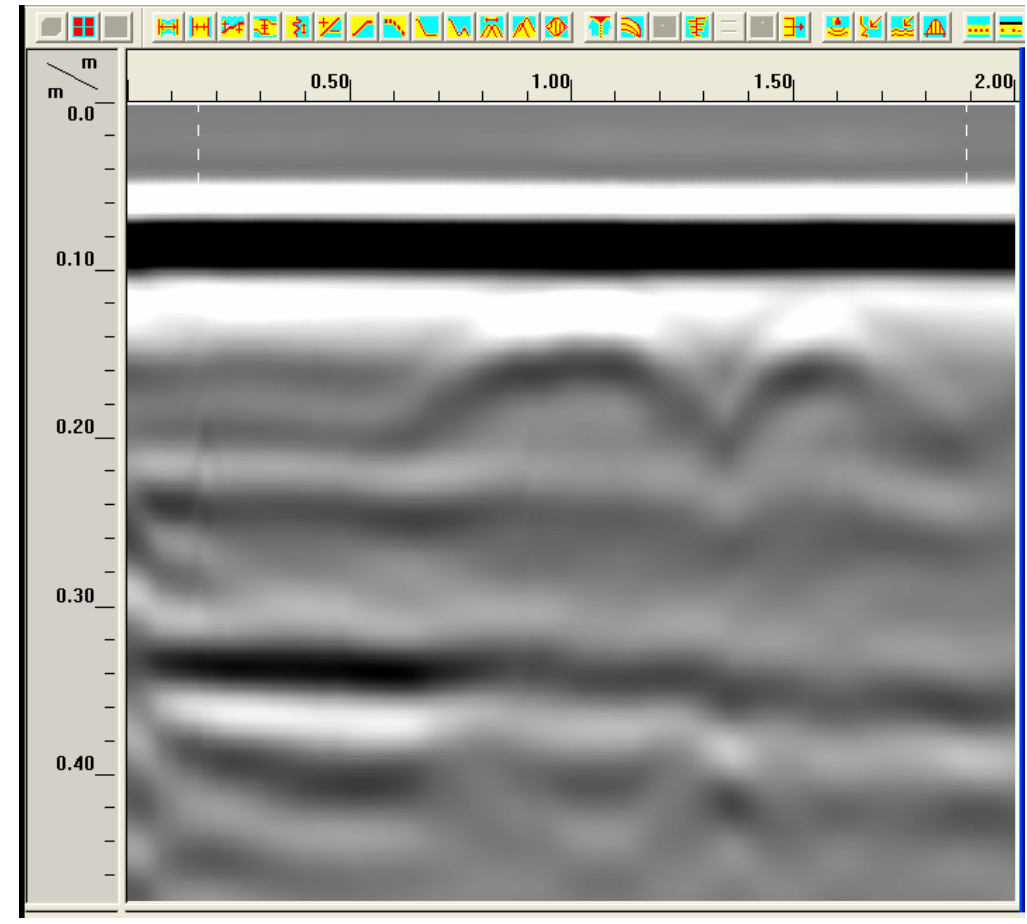

(b)

Figure 5-67 Raw GPR data of Log 7, vertical scale indicating (a) time (ns) (b) depth (m) 


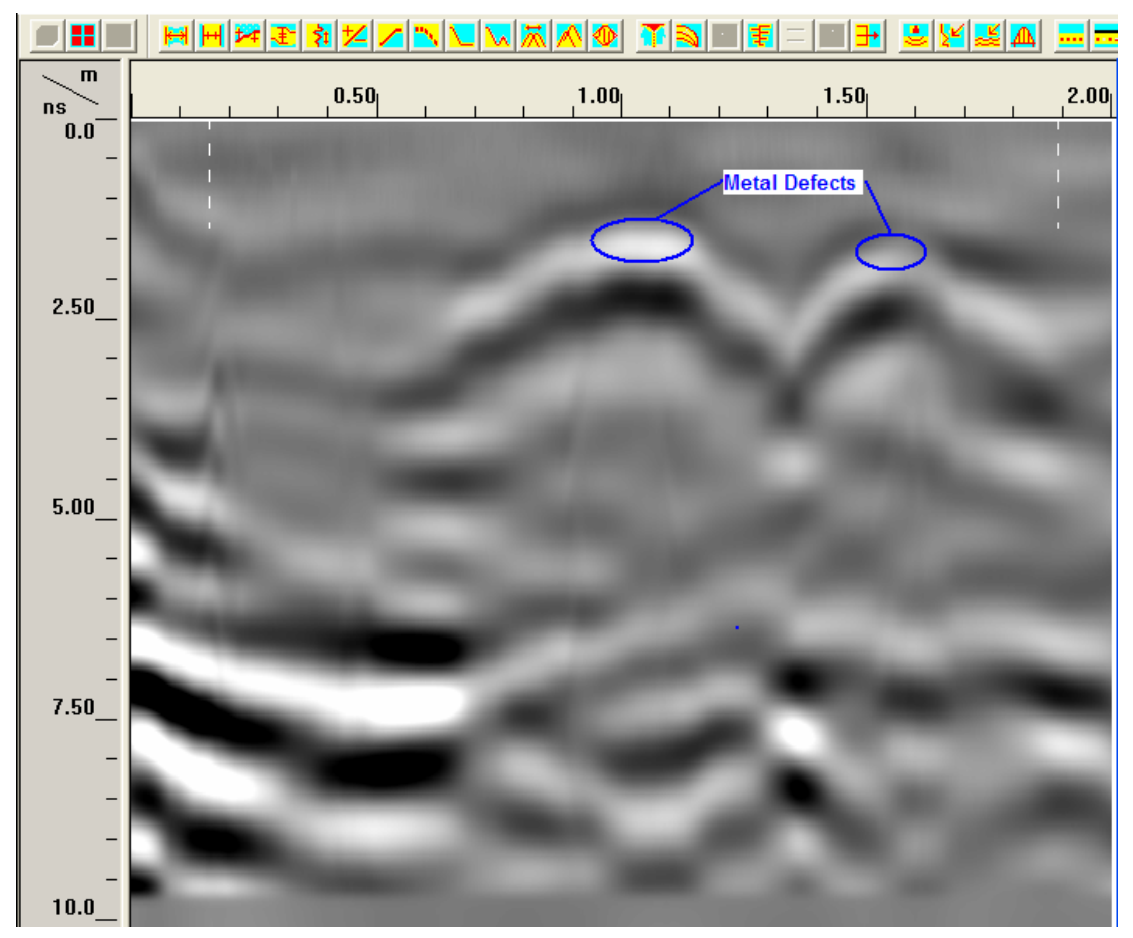

(a)

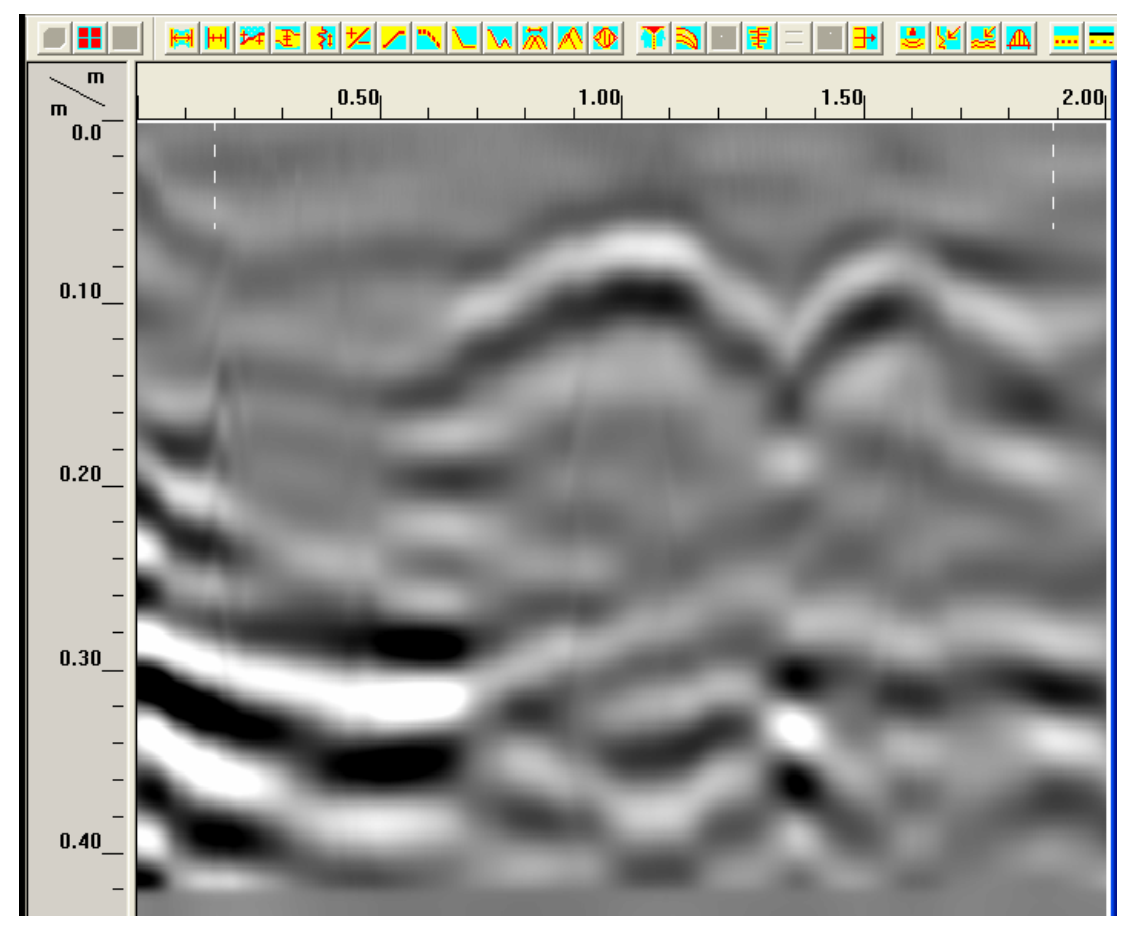

(b)

Figure 5-68 Processed GPR data of Log 7, vertical scale indicating (a) time (ns) (b) depth (m) 


\subsection{INTERFERENCE TESTS}

The defect detection results for the logs discussed earlier relied on single antenna acquisition mode. Scanning of logs several times with log rotation is a time consuming task. A more efficient way of data collection, especially for on-line implementation in saw mills, would be to place multiple antennas around the log and collect the GPR scans simultaneously as the multiple antenna set up is moved along the length of the log. When multiple antennas are used for data acquisition, there is concern regarding interference of one antenna on the data collected by the other. This interference could include interference of EM signals from one antenna and the scattered reflections on the data collected by the other. In addition, the EM signals from one antenna may get reflected by the body of the second antenna, resulting in spurious reflections. To evaluate the extent of such interference, several tests were conducted by placing two $900 \mathrm{MHz}$ antenna opposite to each other and also at right angles. For the purpose of this testing, $\log 4$ was chosen as it had uniform diameter, low moisture content averaging $16 \%$, and had embedded metal defects at known locations that showed distinct parabolic reflections in the GPR scan which made it easier to compare the scans from different antenna configurations. The characteristics of $\log 4$ are given in Table 5.4.

The first test involved testing the log with a single antenna placed on the top as shown in Figure 5-69(a). The data collection parameters for this antenna are shown in Figure 5-69(b). The raw GPR scan collected using this configuration and the corresponding processed GPR scan are given in Figure 5-70 and Figure 5-71.

For the next test, two antennas were placed at right angles to each other as shown in Figure 5-72(a). In this configuration, the top antenna was maintained at a fixed height and was connected to the GPR mainframe for collecting data. The side antenna was not connected to the GPR mainframe, and the spacing between the antenna and the log was varied for different tests. These tests were conducted to evaluate the influence of the side antenna's housing on the data collected by the top antenna. The various configurations involving different spacing between the side antenna and the $\log (0.03 \mathrm{~m}, 0.1 \mathrm{~m}$, and 0.15 $\mathrm{m}$ ) and the corresponding processed GPR data from the top antenna are shown in Figures 5-72, 5-73 and 5-74. Figure 5-75 shows the test configuration and processed GPR data 
for the top antenna with the side antenna completely removed. This test configuration was same as that shown in Figure 5-69(a), and the corresponding processed data was the same as in Figure 5-71(a). After comparing the processed GPR scans from all the above tests as shown in Figures 5-72(b), 5-73(b), 5-74(b) and 5-75(b), it was found that all scans were identical in nature. Thus, it was concluded that there was no interference in the GPR data collected by the top antenna due to the presence of the side antenna's housing.

The next set of tests were conducted to evaluate the extent of electromagnetic interference from the signals sent by one antenna on the data collected by another antenna, when the antennas are used simultaneously in the data acquisition mode. The first test in this series involved collection of GPR data using the side antenna with the top antenna completely removed as shown in Figure 5-76. The acquired raw GPR data is shown in Figure 5-77 and the corresponding processed data are shown in Figure 5-78. In the next test, data was collected using both top and side antennas in multi-channel mode as shown in Figure 5-79. The raw and processed data for this configuration are shown in Figure 5-80 and Figure 5-81, respectively. In each of these figures, the top part of the scan is for the top antenna and the bottom part is for the side antenna.

The data from the two channel antenna configuration (both top and side antenna) was compared to the single channel antenna configurations (only top antenna and only side antenna). The data from the top antenna in the two channel configuration was exactly similar to the data from the single top antenna configuration. Also, the data from the side antenna from the two channel configuration mode was similar to the data from the single side antenna configuration. The only observable differences between Figure 578 and 5-81 for side antenna are due to differences in display gain, but the characteristics of the display gain are the same. From these data comparison, it was concluded that neither the antenna body nor the electromagnetic signals of one antenna interferes with that of another antenna.

The next set of tests involved placing the two antennas opposite to each other as shown in Figure 5-82. In the first test of this series, the data was collected in the T1R1T2R2 mode. Here "1" and "2" refer to the two antennas and each antenna is transmitting 
(T) and receiving (R) its own signal. The collected raw data and the processed data for this particular test are given in Figures 5-83 and 5-84, respectively. In these figures, the side antenna to the right in Figure 5-82 was connected to Channel "1' and the one on the left side to Channel "2". So the top part of Figures 5-84(a) and 5-84(b) are for the side antenna to the right. This when compared to the case when the side antenna was used alone (Figure 5-78), shows that there are some differences in these figures in terms of the where the signal clutters appear, and this issue needs further investigation.

The next test involved a similar antenna configuration (two antennas facing each other (Figure 5-85) except that data was collected in the T1R2-T2R1 mode. Here the signal transmitted by antenna "1" (right antenna) was received by antenna "2" (left antenna). At the same time, antenna "1" received the signal transmitted by antenna " 2 ". The collected raw and processed GPR data are shown in Figures 5-86 and 5-87, respectively. Comparison of the GPR data in Figures 5-84 and 5-87 shows that there are some similarities as well as differences. The differences are expected since Figure 5-84 is for the mono-static mode (transmitting and receiving antenna on the same side) while Figure 5-87 is for the bi-static mode (one antenna transmitting and the other antenna located on the opposite side of the log receiving the signal). Analysis of the data from the bi-static mode needs further consideration, which is outside the scope of this research. 


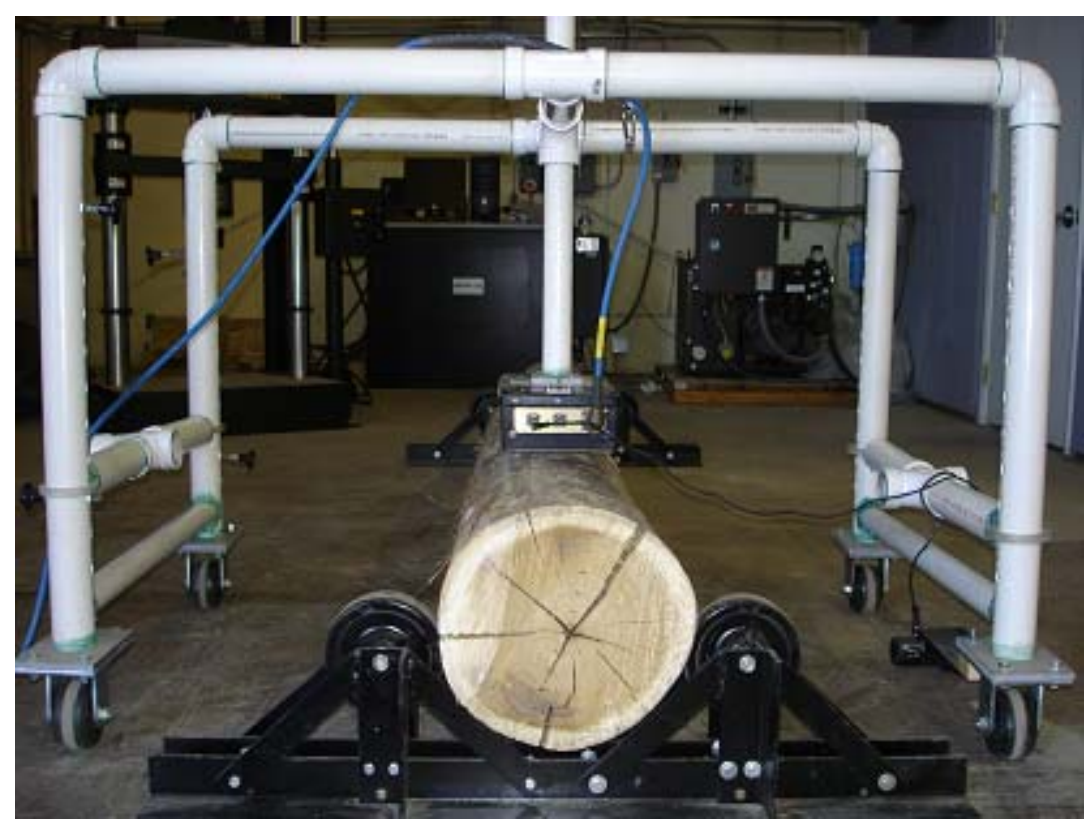

(a)

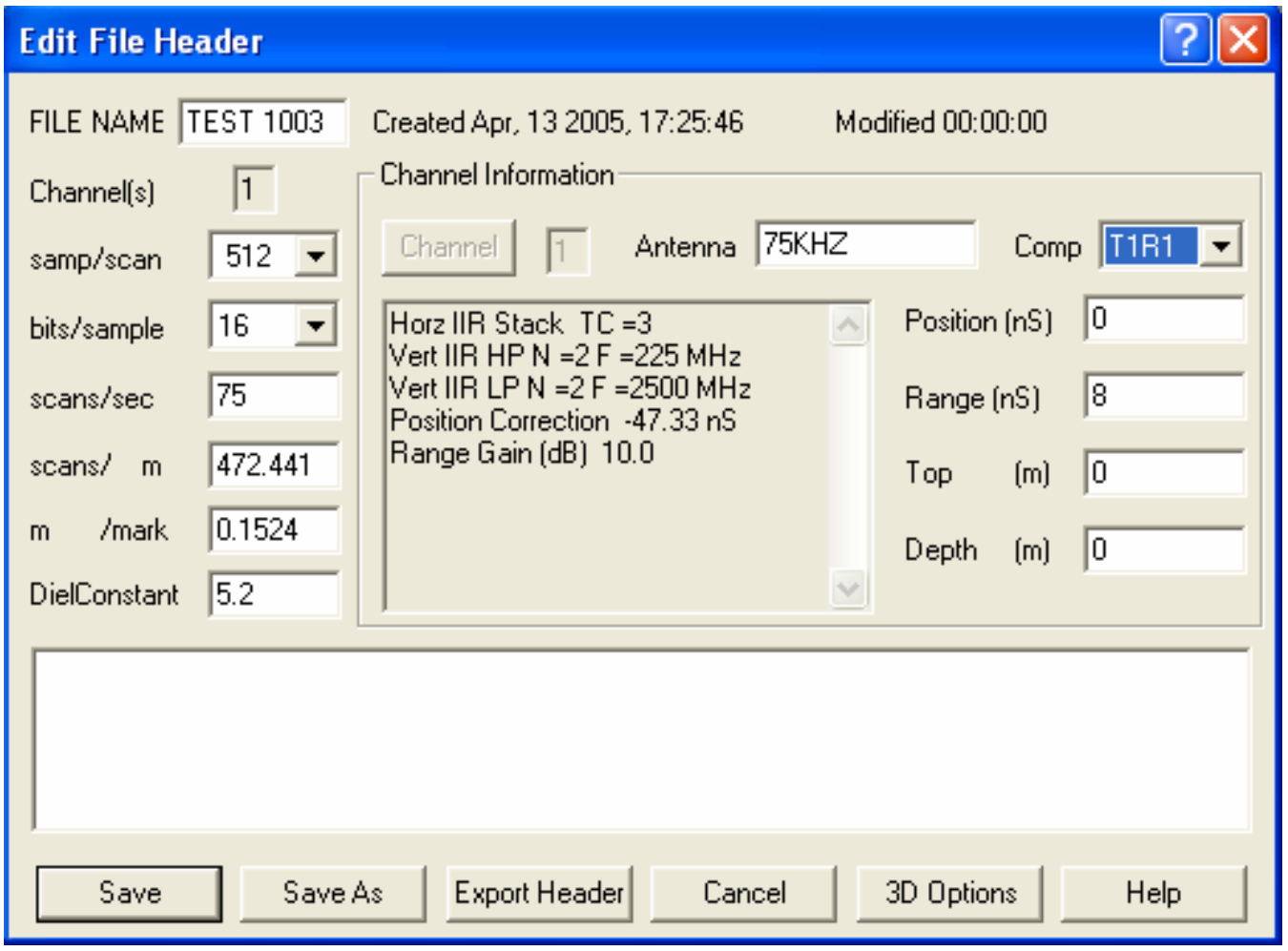

(b)

Figure 5-69 (a) Top antenna configuration and its (b) data collection parameters 


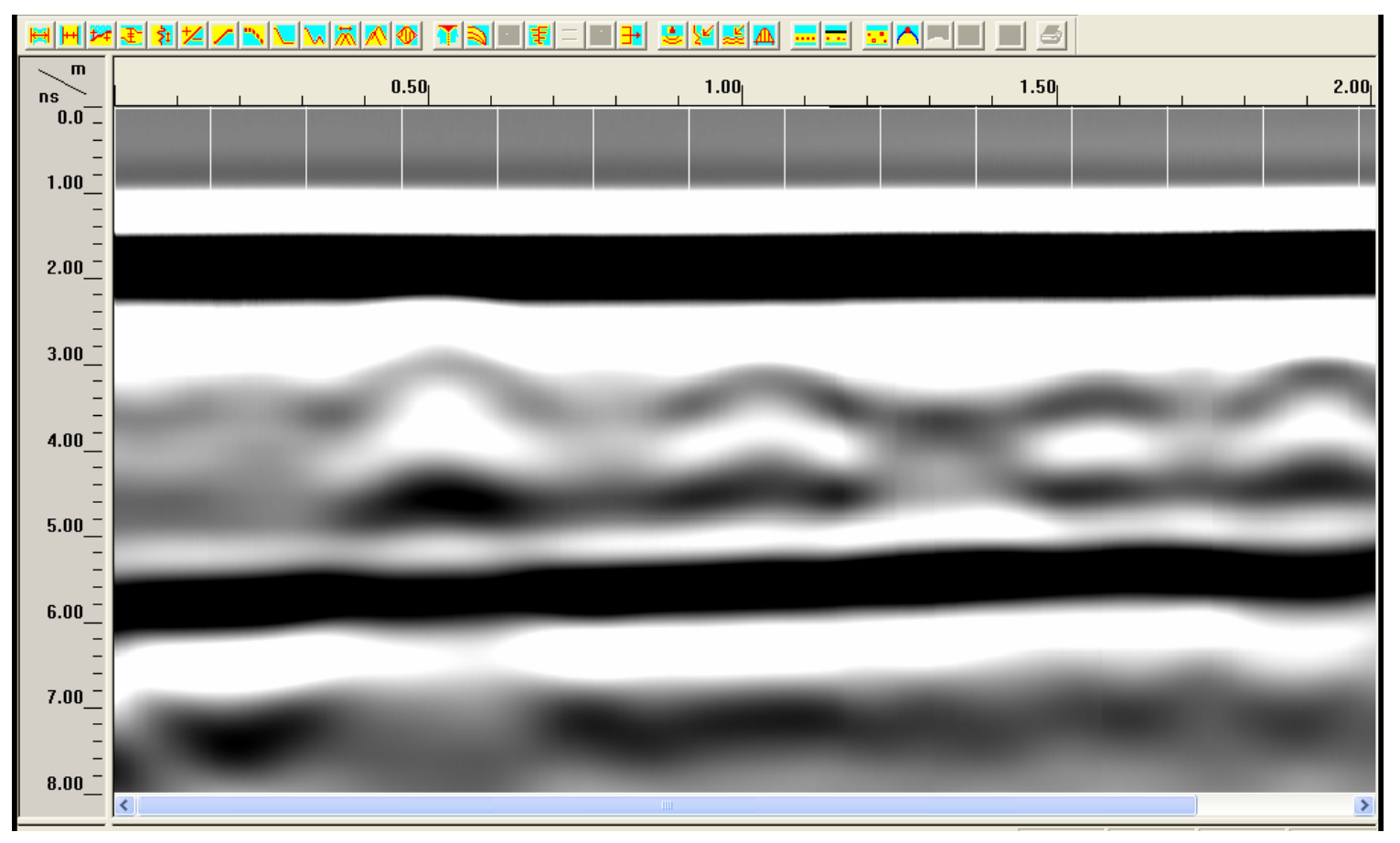

(a)

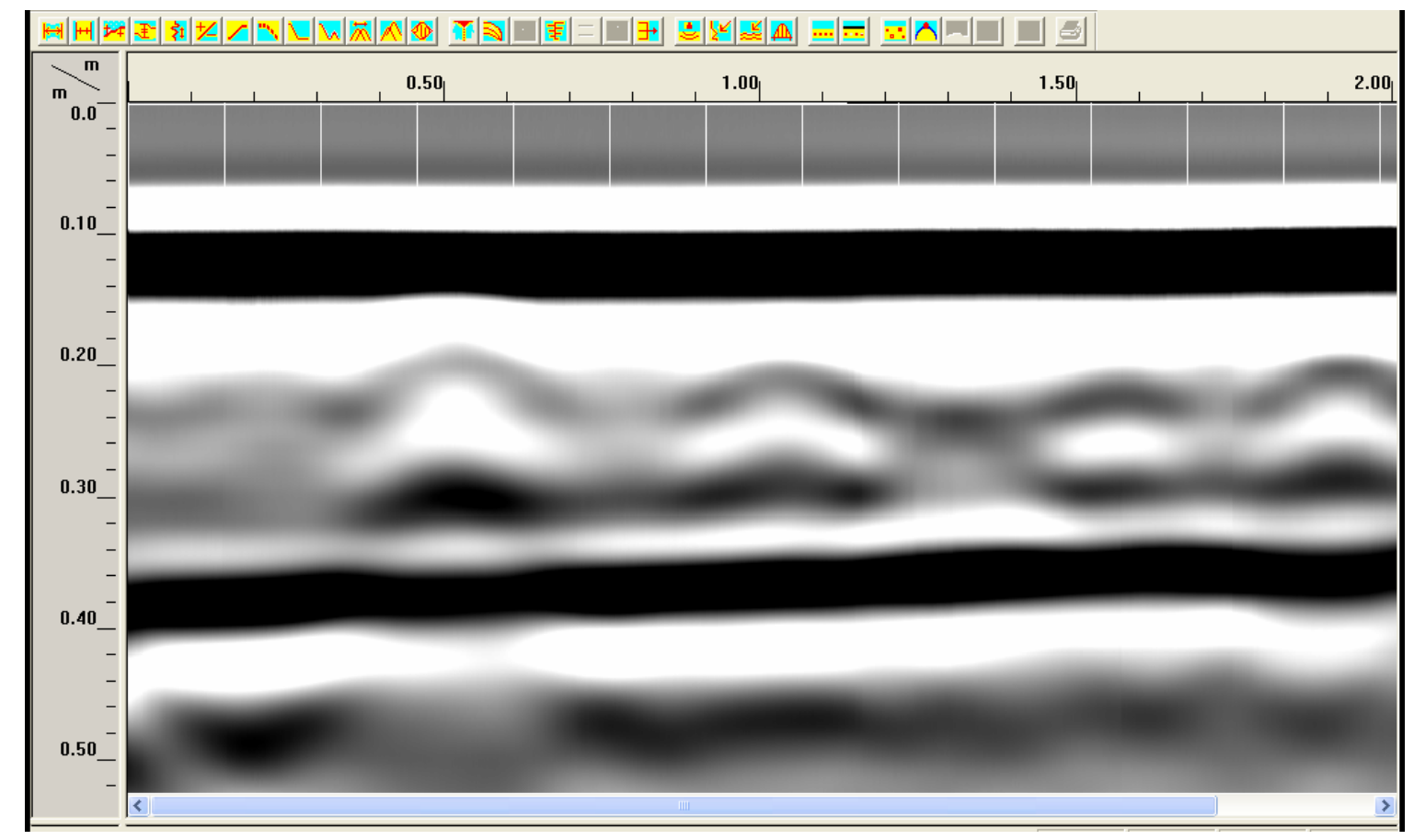

(b)

Figure 5-70 Raw GPR data for the antenna configuration shown in Figure 5.69, horizontal scale indicating the travel distance along the length of the $\log (\mathrm{m})$ and vertical scale indicating (a) time (ns) (b) depth (m) 


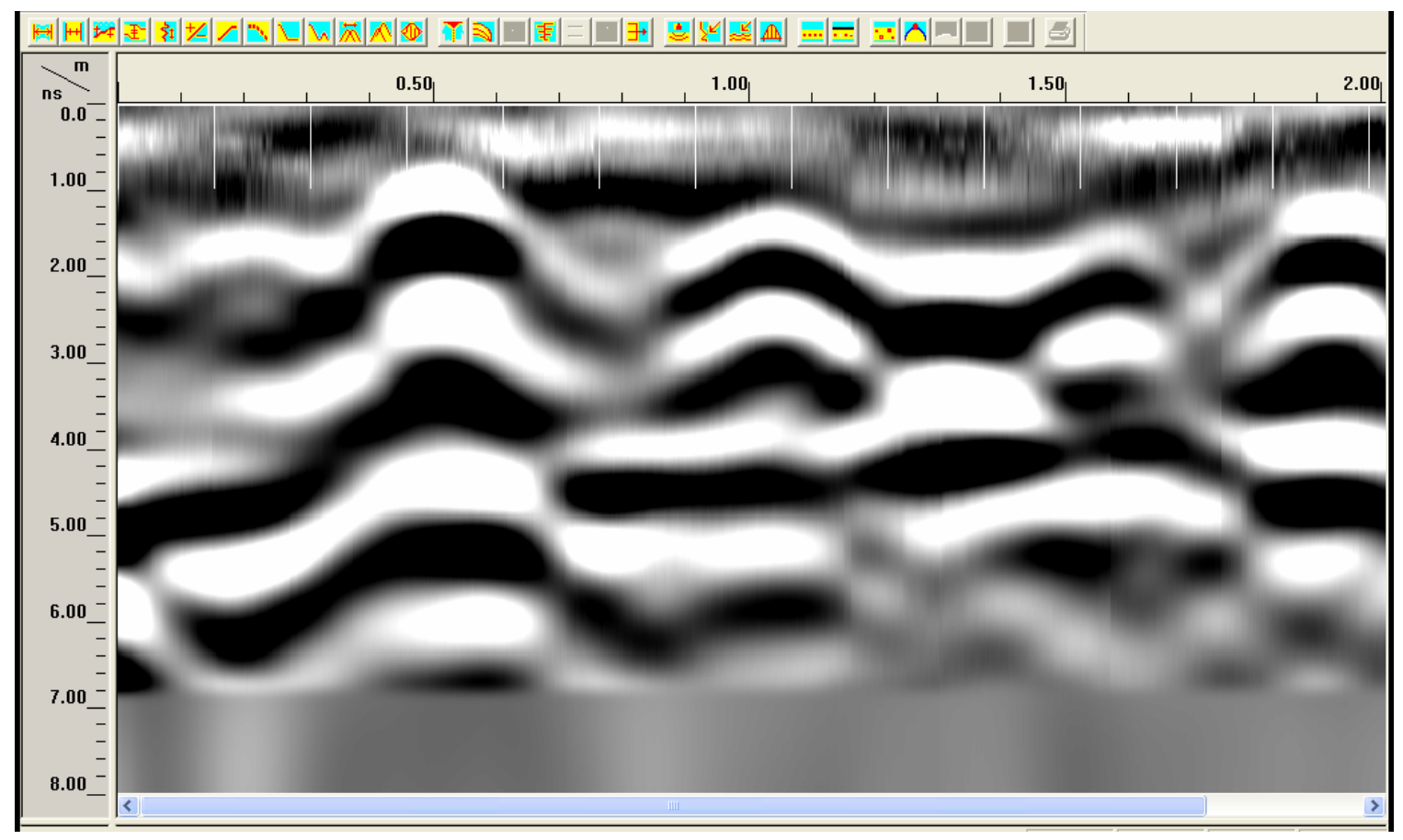

(a)

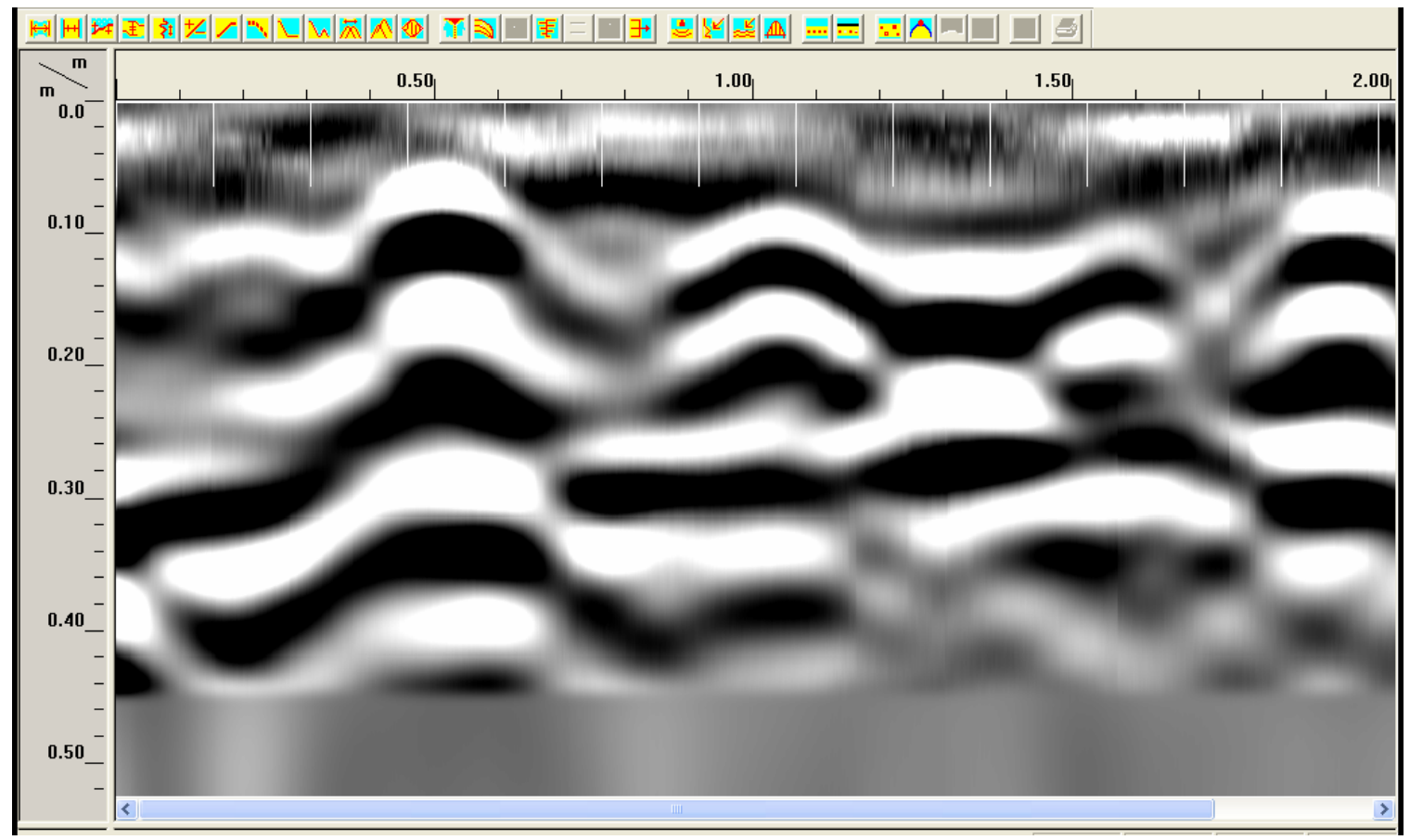

(b)

Figure 5-71 Processed GPR data for the antenna configuration shown in Figure 5.69, horizontal scale indicating the travel distance along the length of the $\log (\mathrm{m})$ and vertical scale indicating (a) time (ns) (b) depth (m) 


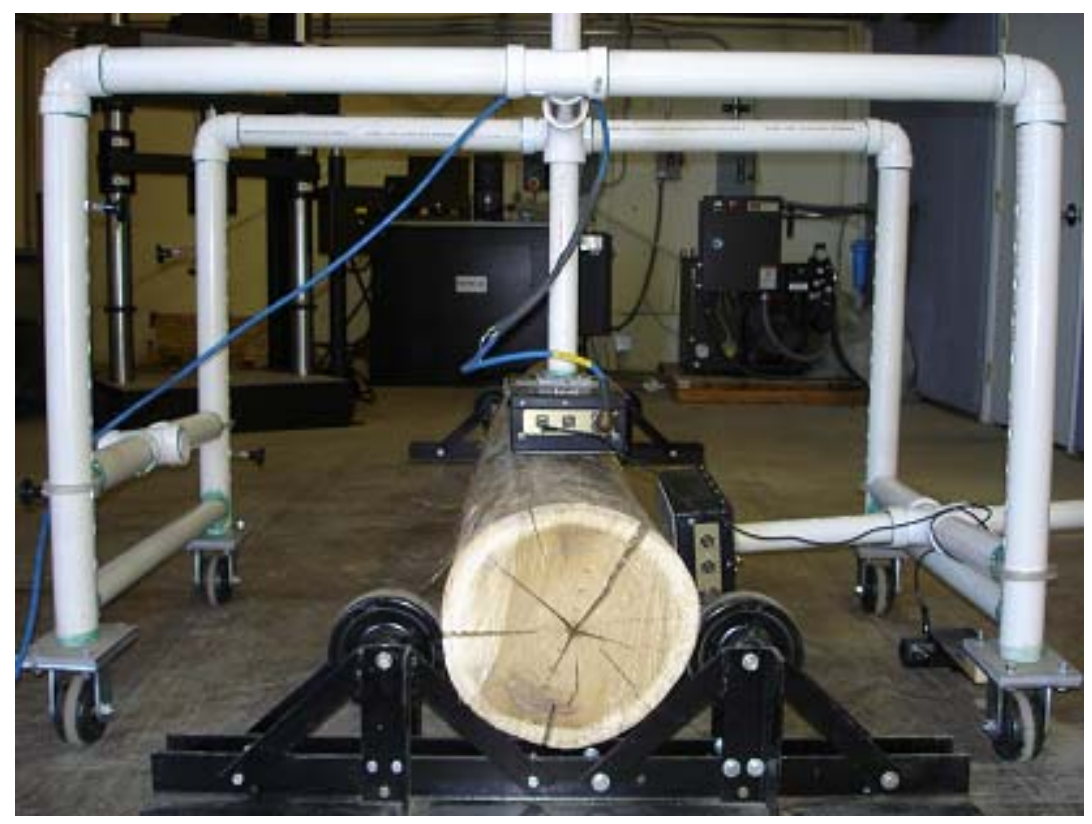

(a)

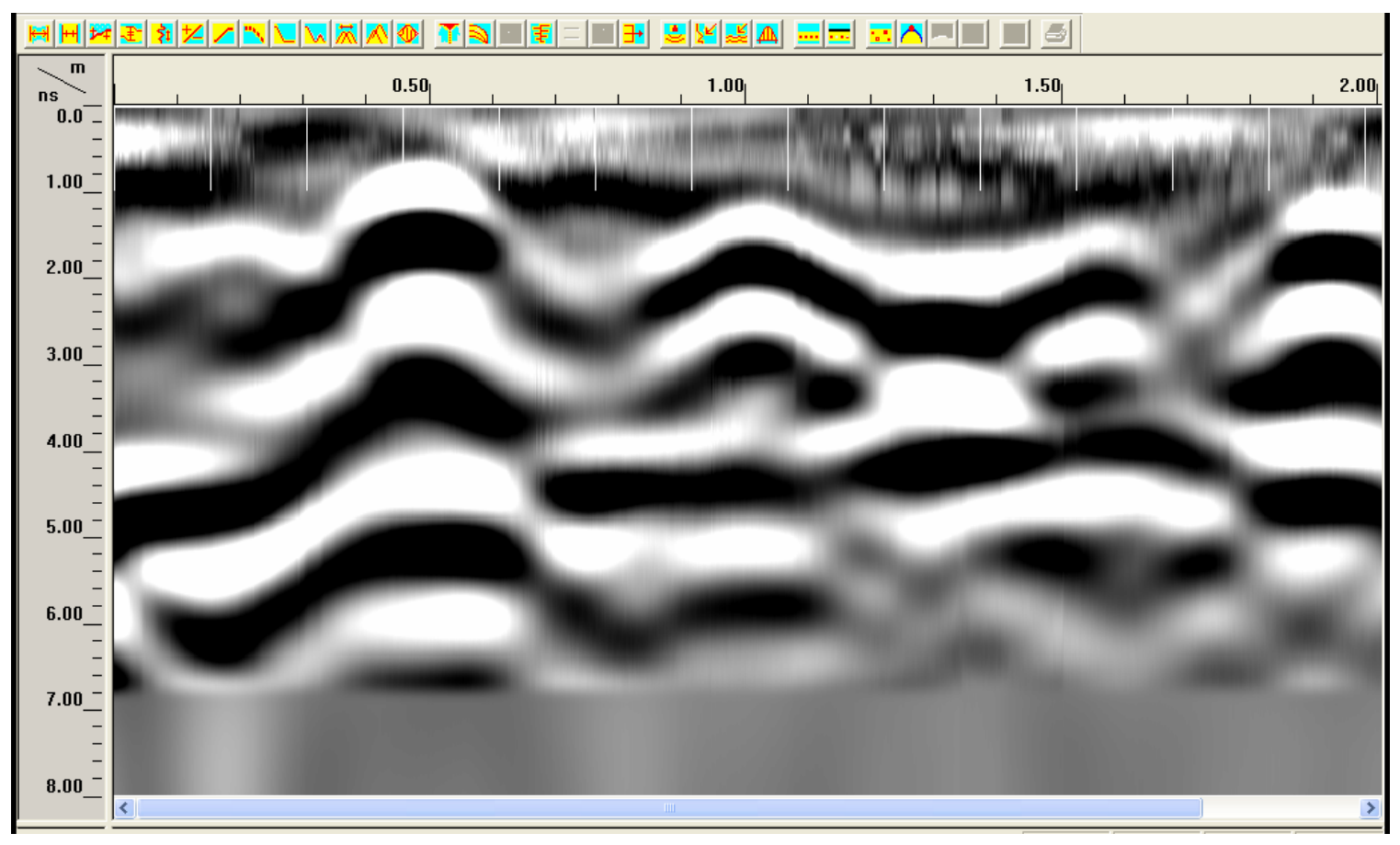

(b)

Figure 5-72 Antenna on the top surface collecting data with side antenna at a distance $0.03 \mathrm{~m}$ from the log surface (b) processed data for the antenna configuration shown in (a) 


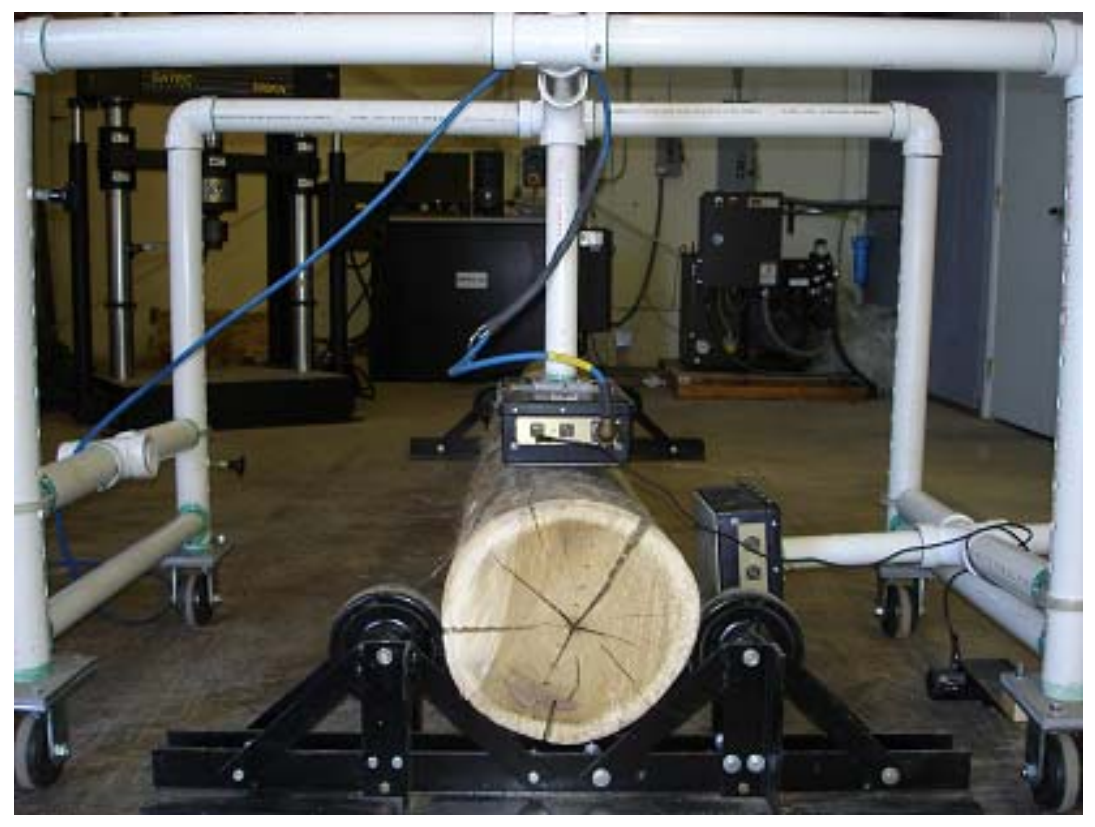

(a)

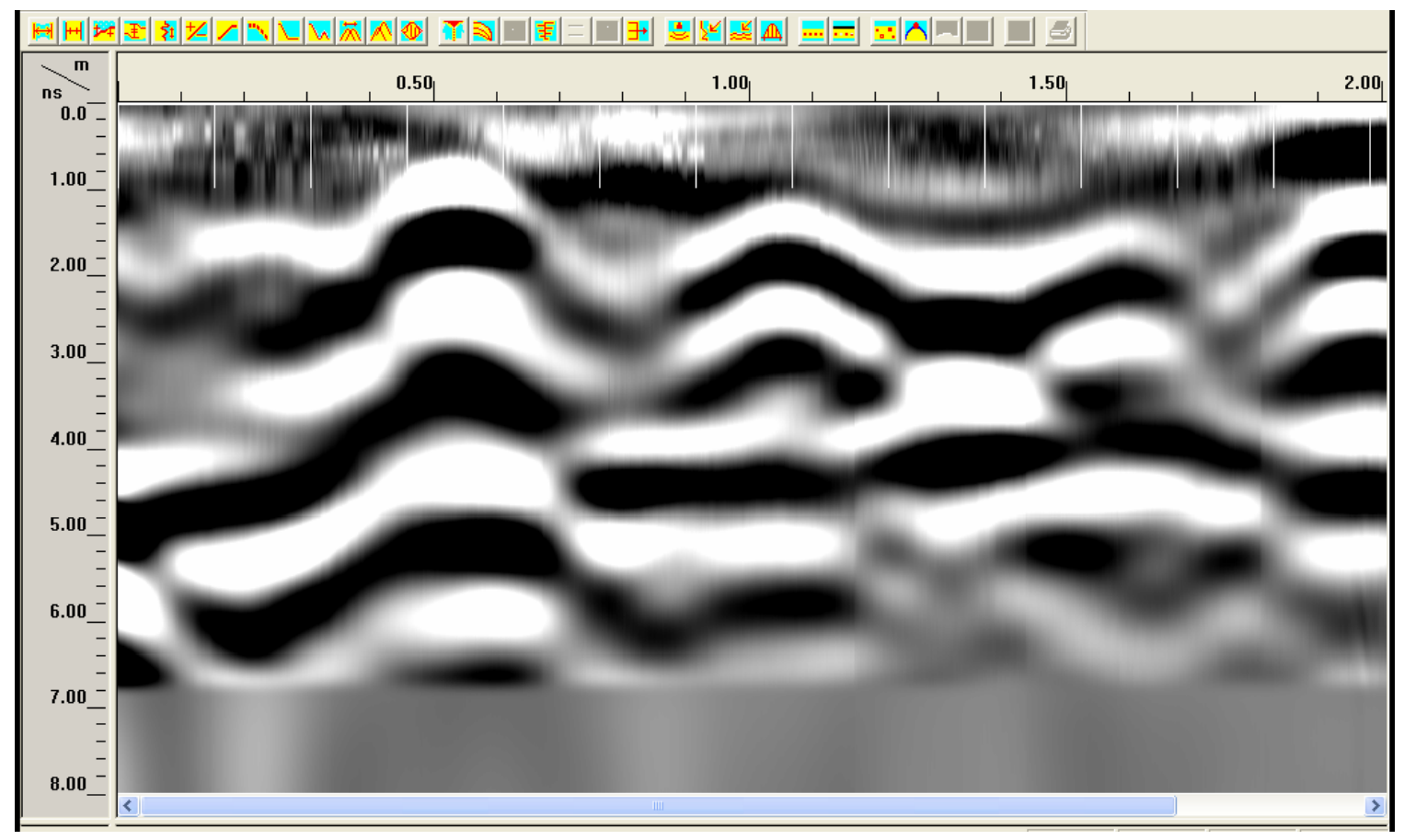

(b)

Figure 5-73 Antenna on the top surface collecting data with side antenna at a distance $0.1 \mathrm{~m}$ from the log surface (b) processed data for the antenna configuration shown in (a) 


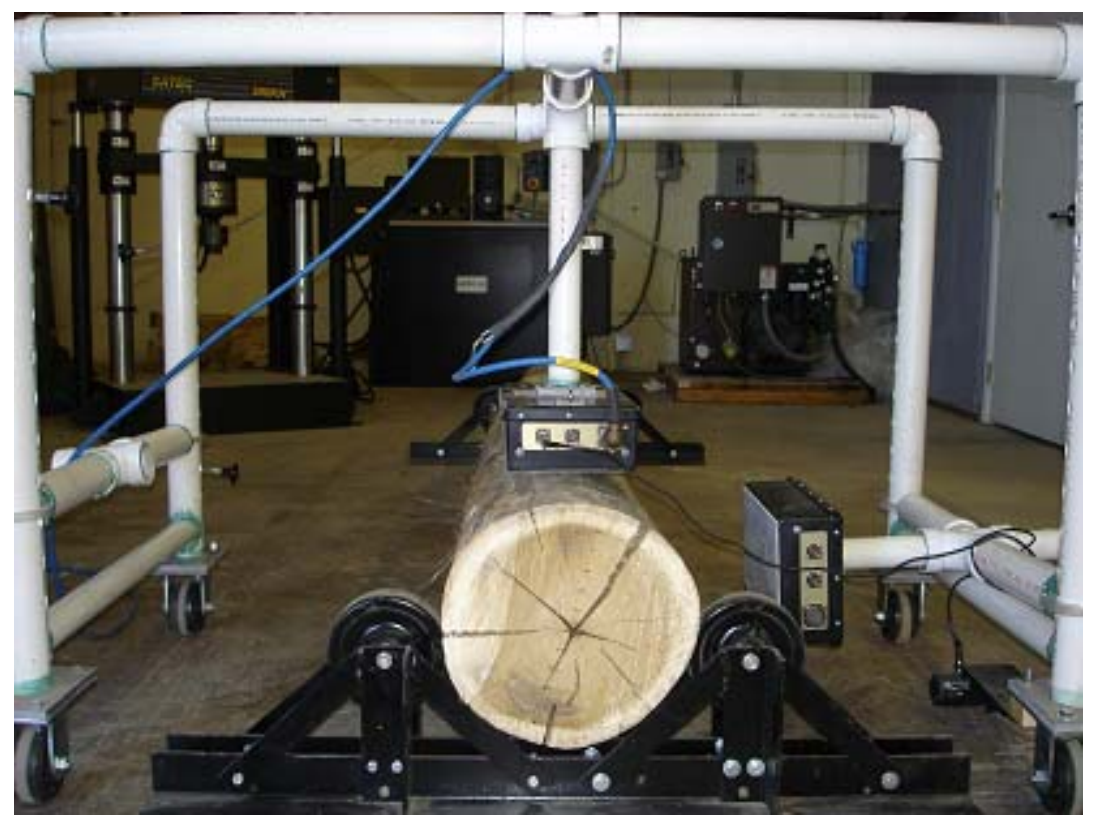

(a)

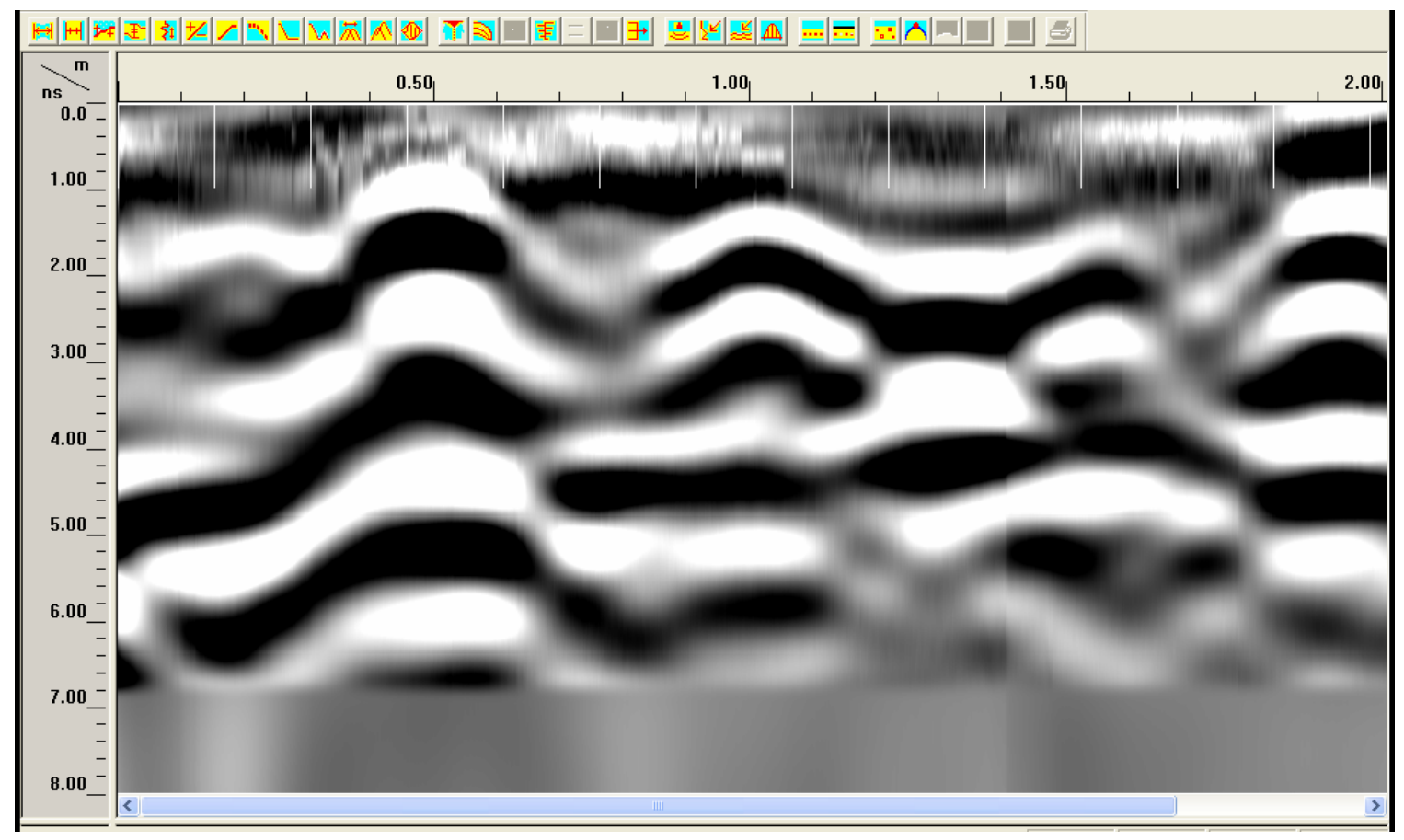

(b)

Figure 5-74 Antenna on the top surface collecting data with side antenna at a distance $0.15 \mathrm{~m}$ from the log surface (b) processed data for the antenna configuration shown in (a) 


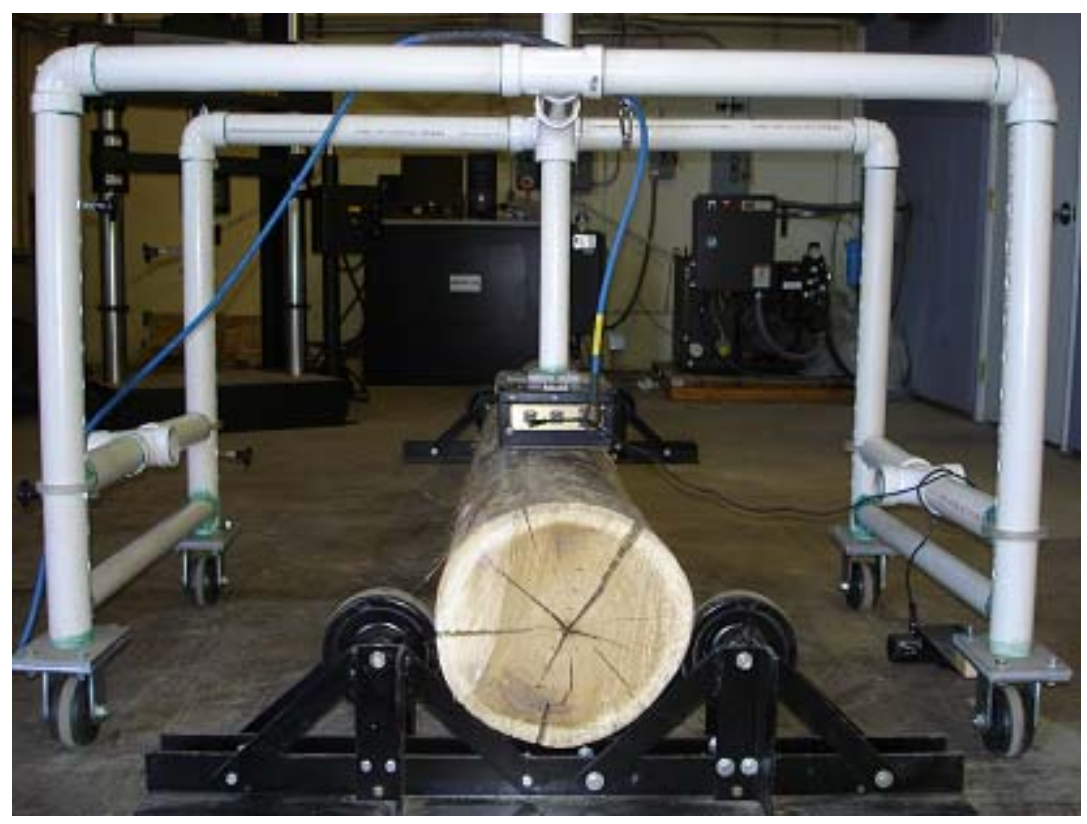

(a)

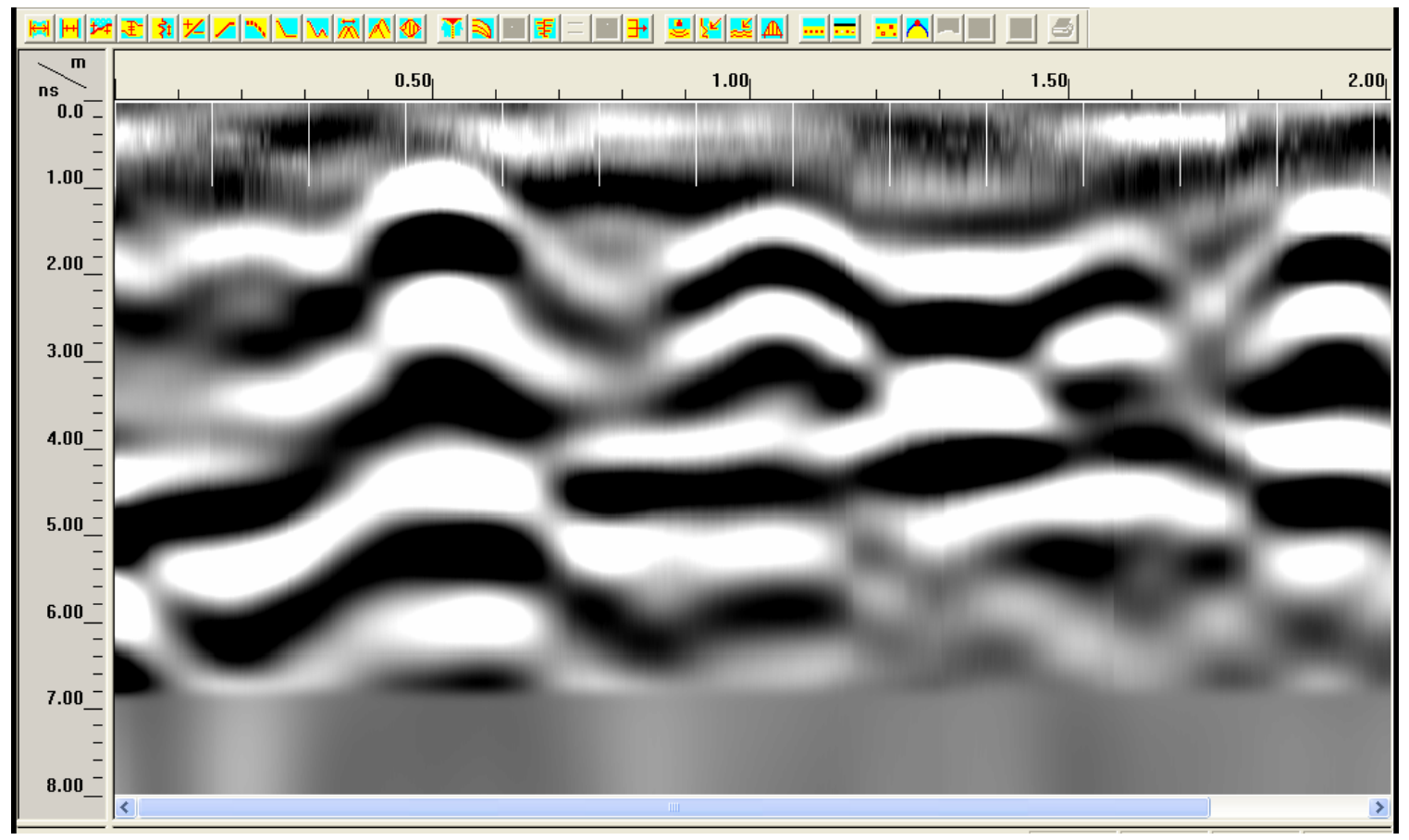

(b)

Figure 5-75 Antenna on the top surface collecting data with side antenna completely removed (b) processed data for the antenna configuration shown in (a) 


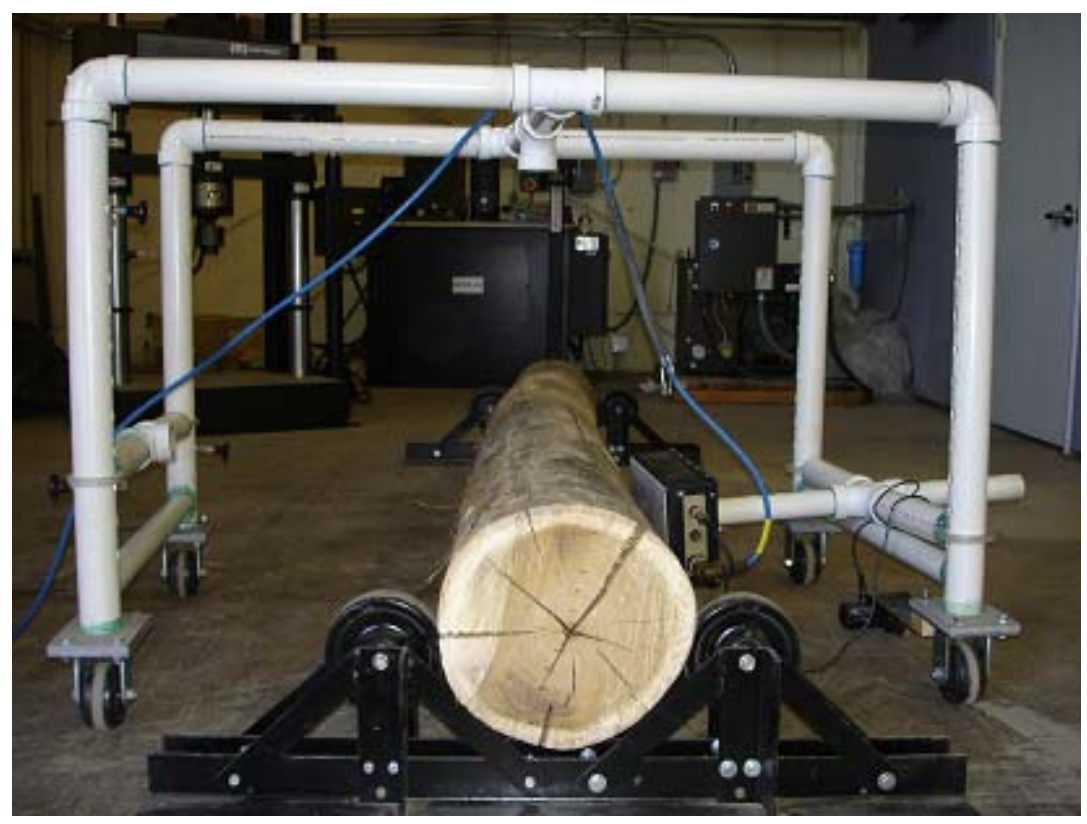

Figure 5-76 Antenna on the top completely removed and single antenna on the side collecting data

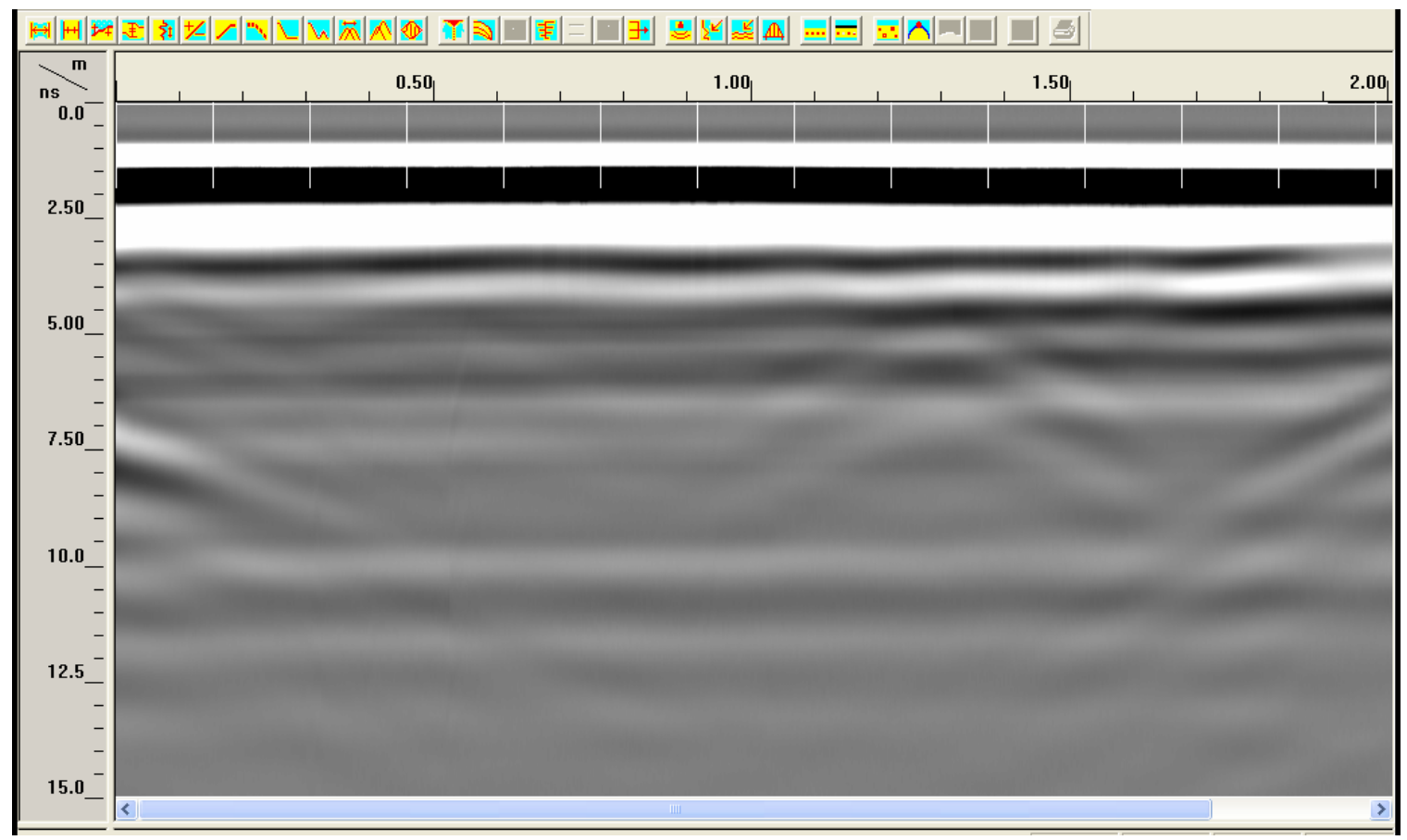

Figure 5-77 Raw GPR data for the antenna configuration shown in Figure 5-76, horizontal scale indicating the travel distance along the length of the $\log (\mathrm{m})$ and vertical scale indicating time (ns) 


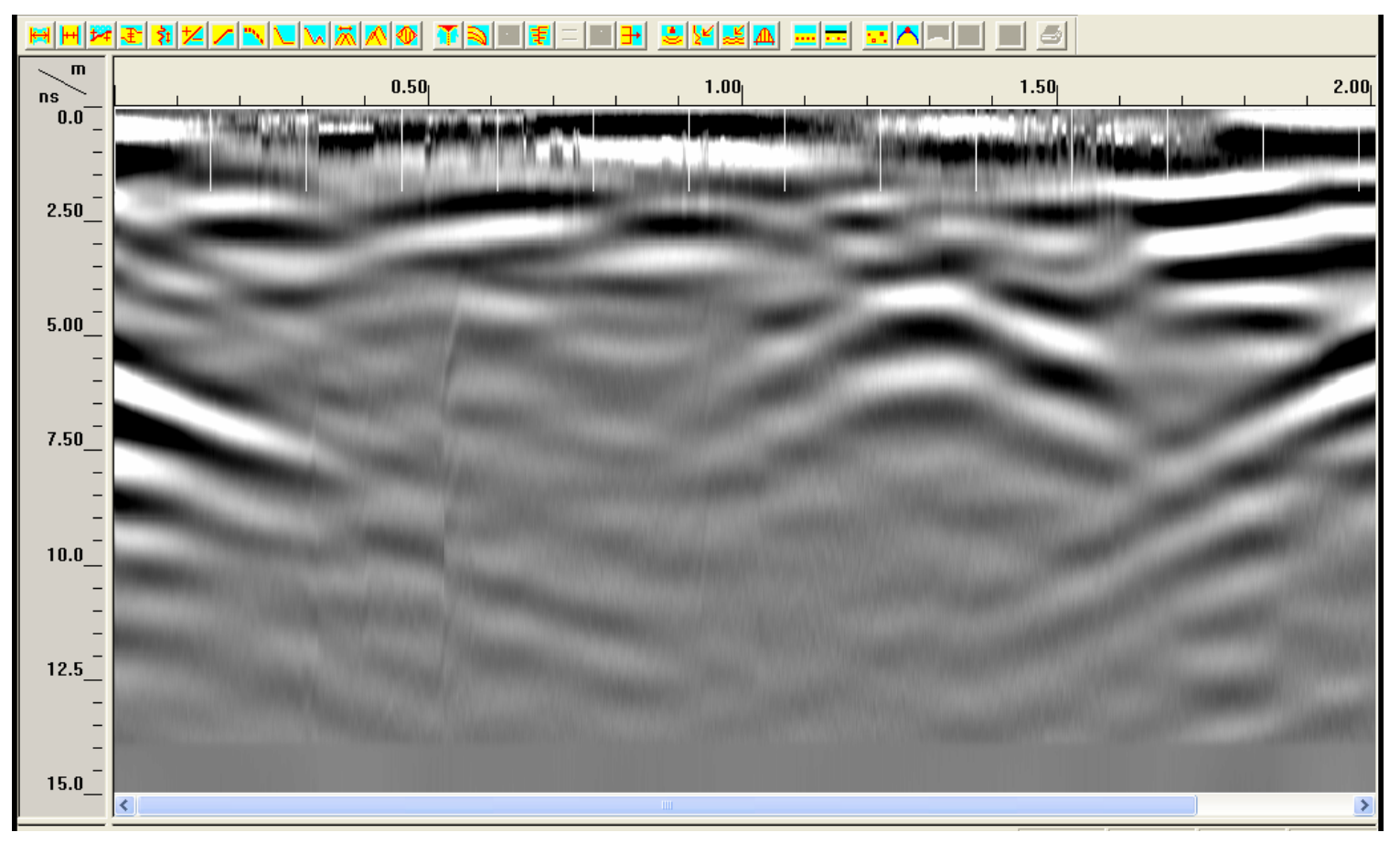

(a)

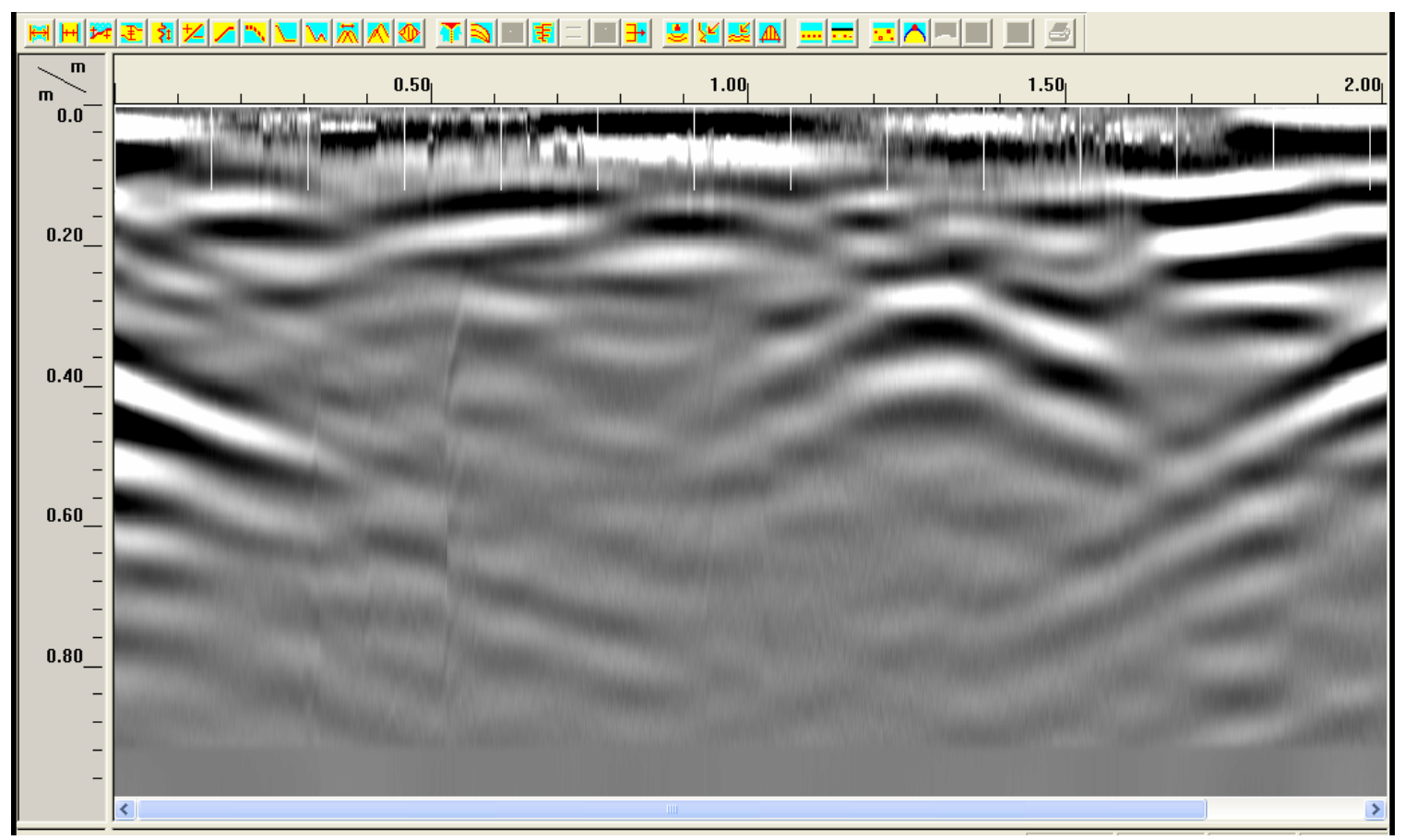

(b)

Figure 5-78 Processed GPR data for the antenna configuration shown in Figure 5-76, horizontal scale indicating the travel distance along the length of the $\log (\mathrm{m})$ and vertical scale indicating

(a) time (ns) (b) depth (m) 


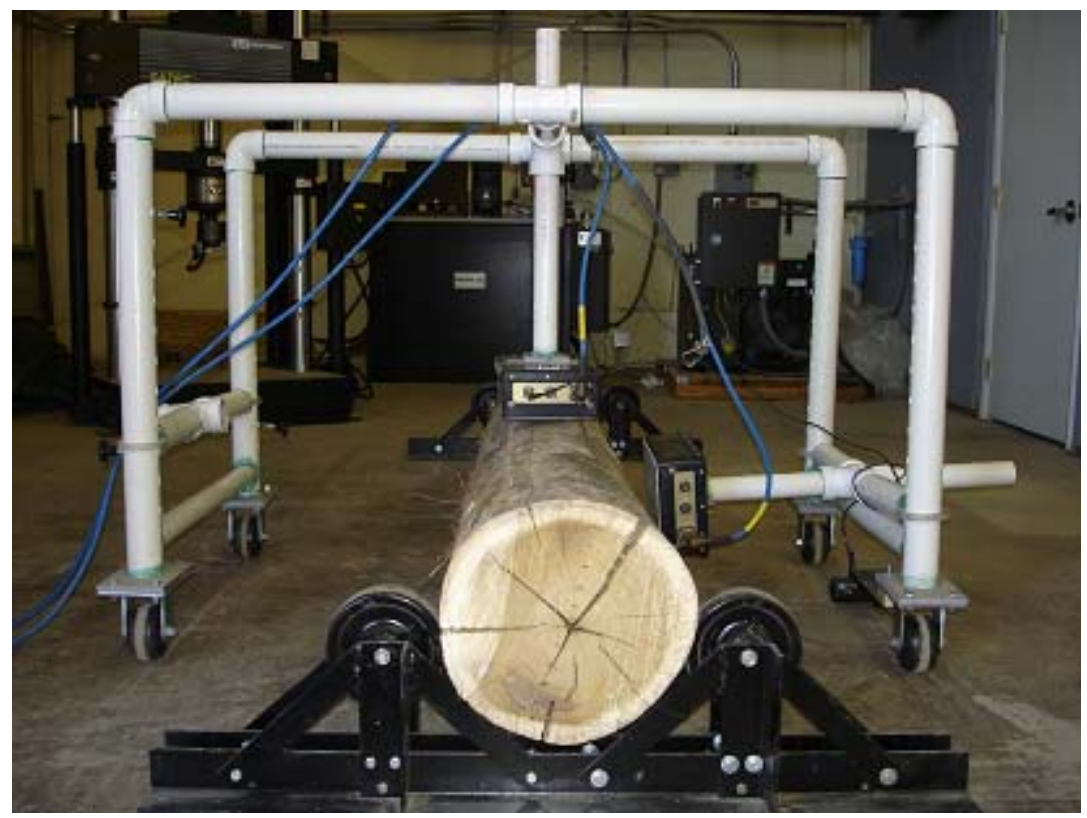

Figure 5-79 Antenna configuration for two channel data collection

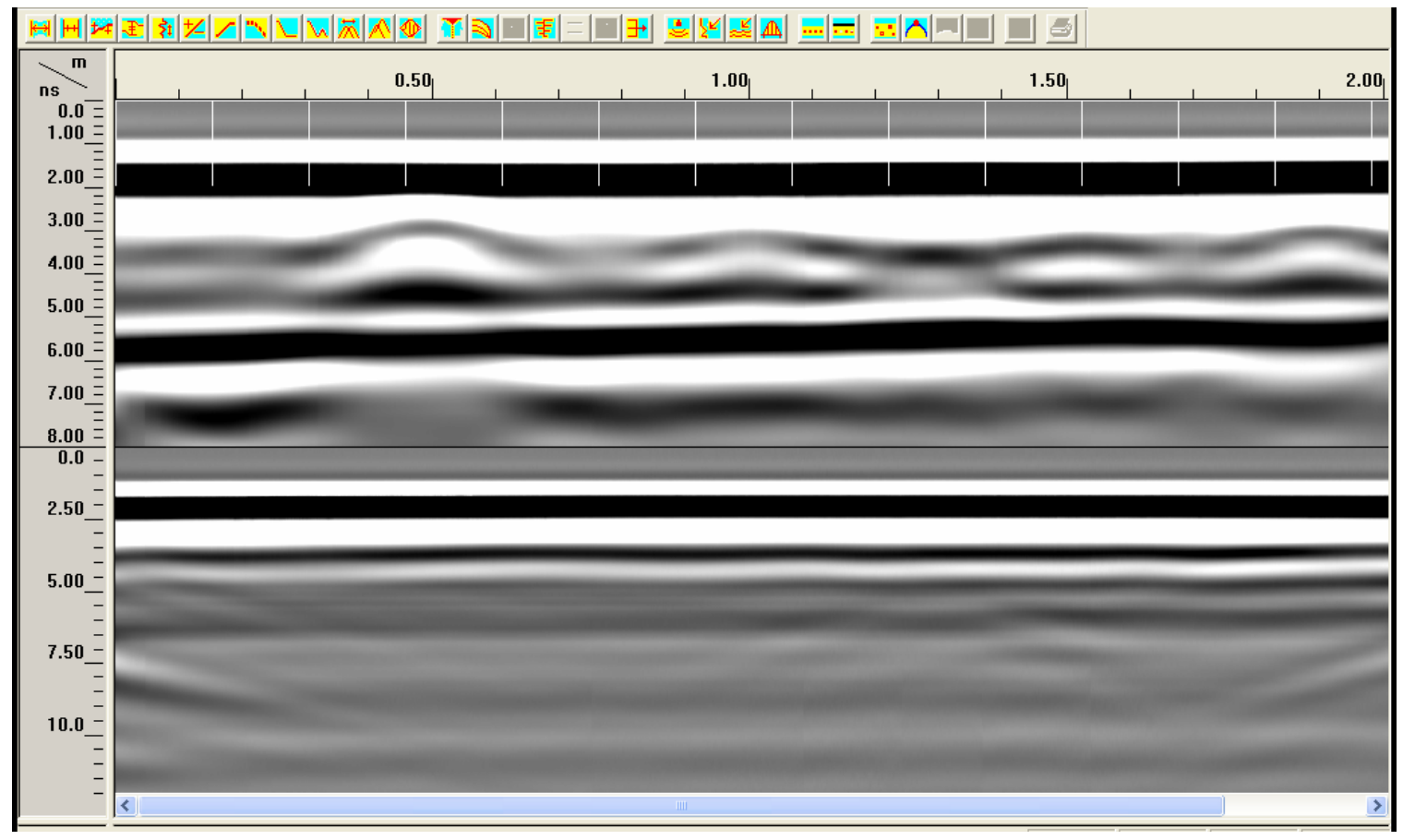

Figure 5-80 Raw GPR data for the antenna configuration shown in Figure 5-79, horizontal scale indicating the travel distance along the length of the $\log (\mathrm{m})$ and vertical scale indicating time (ns) 


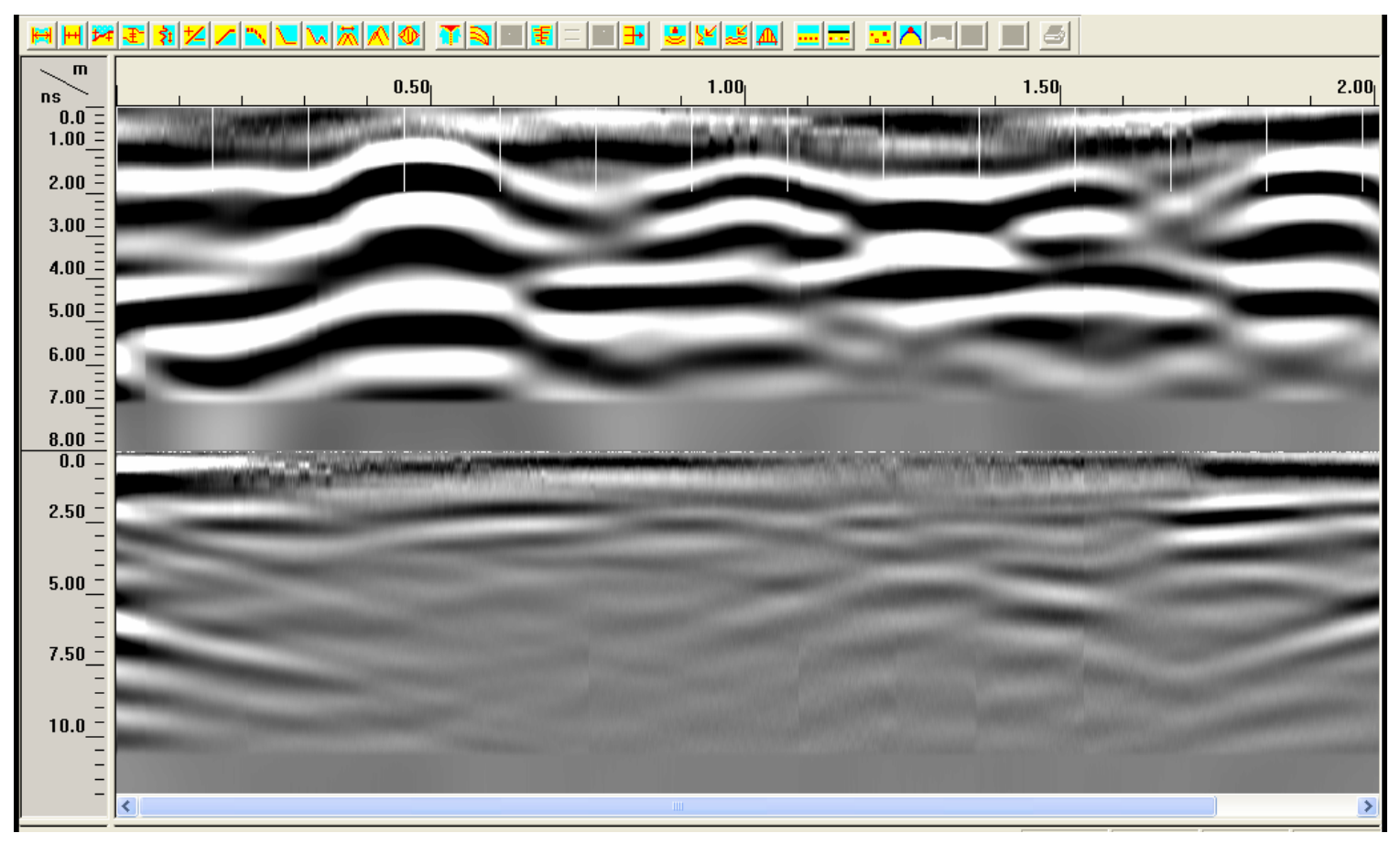

(a)

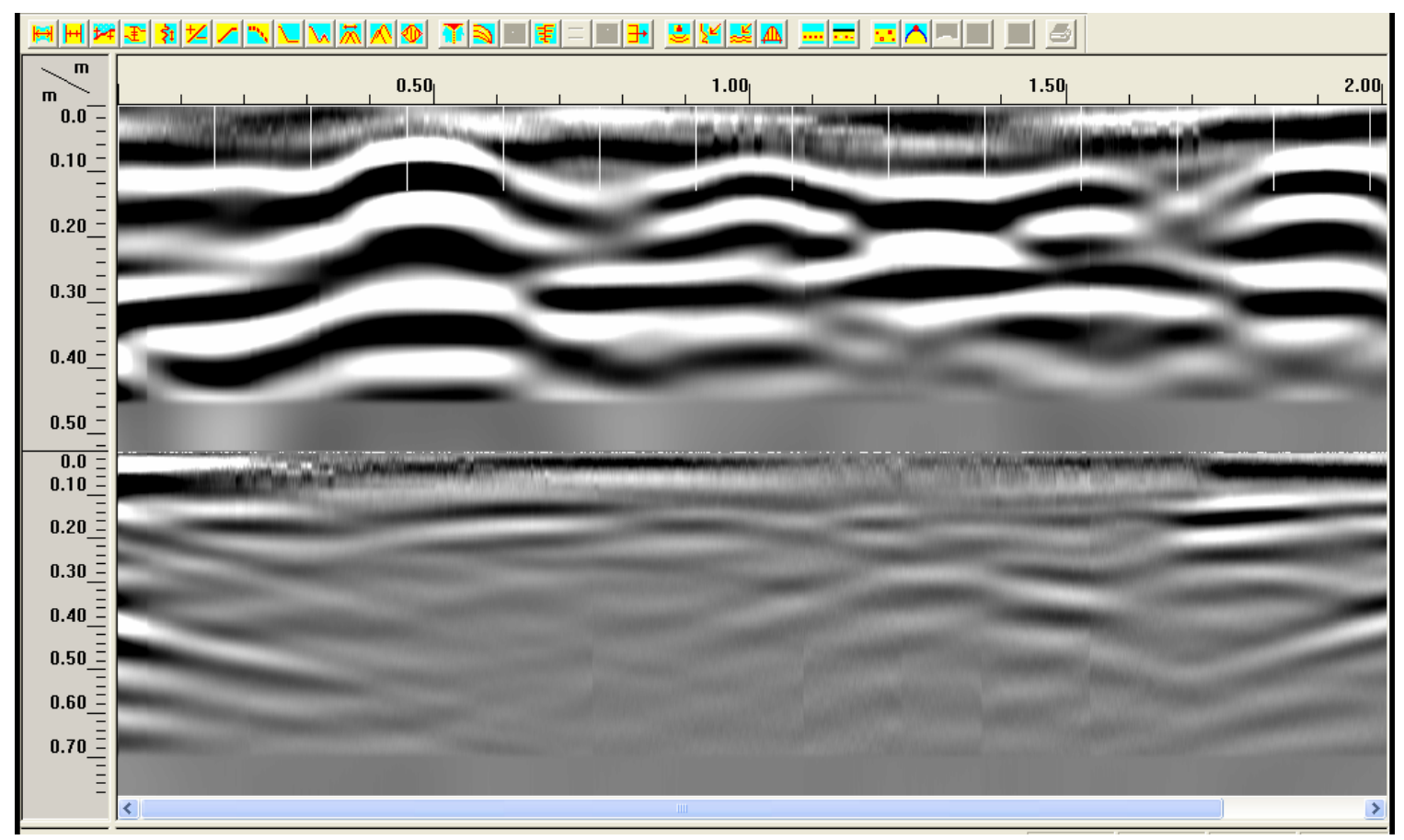

(b)

Figure 5-81 Processed GPR data for the antenna configuration shown in Figure 5-79, horizontal scale indicating the travel distance along the length of the $\log (\mathrm{m})$ and vertical scale indicating

(a) time (ns) (b) depth (m) 


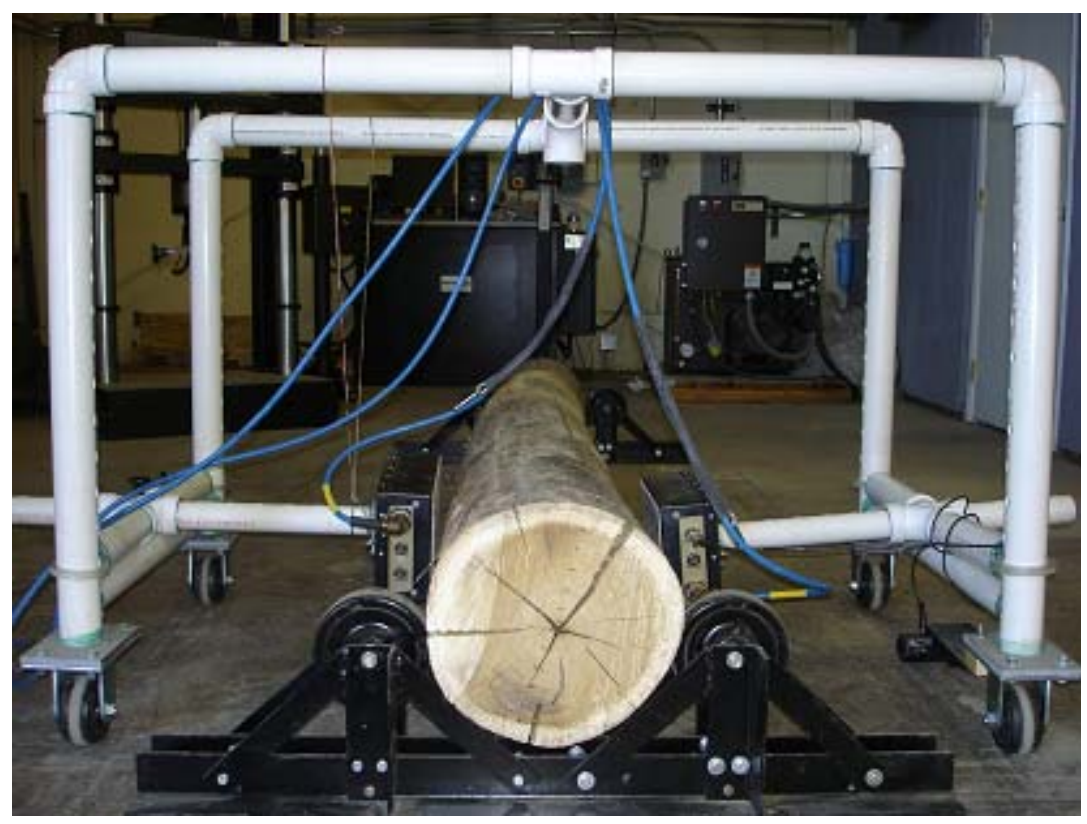

Figure 5-82 Two antenna on the side in T1R1 and T2R2 mode

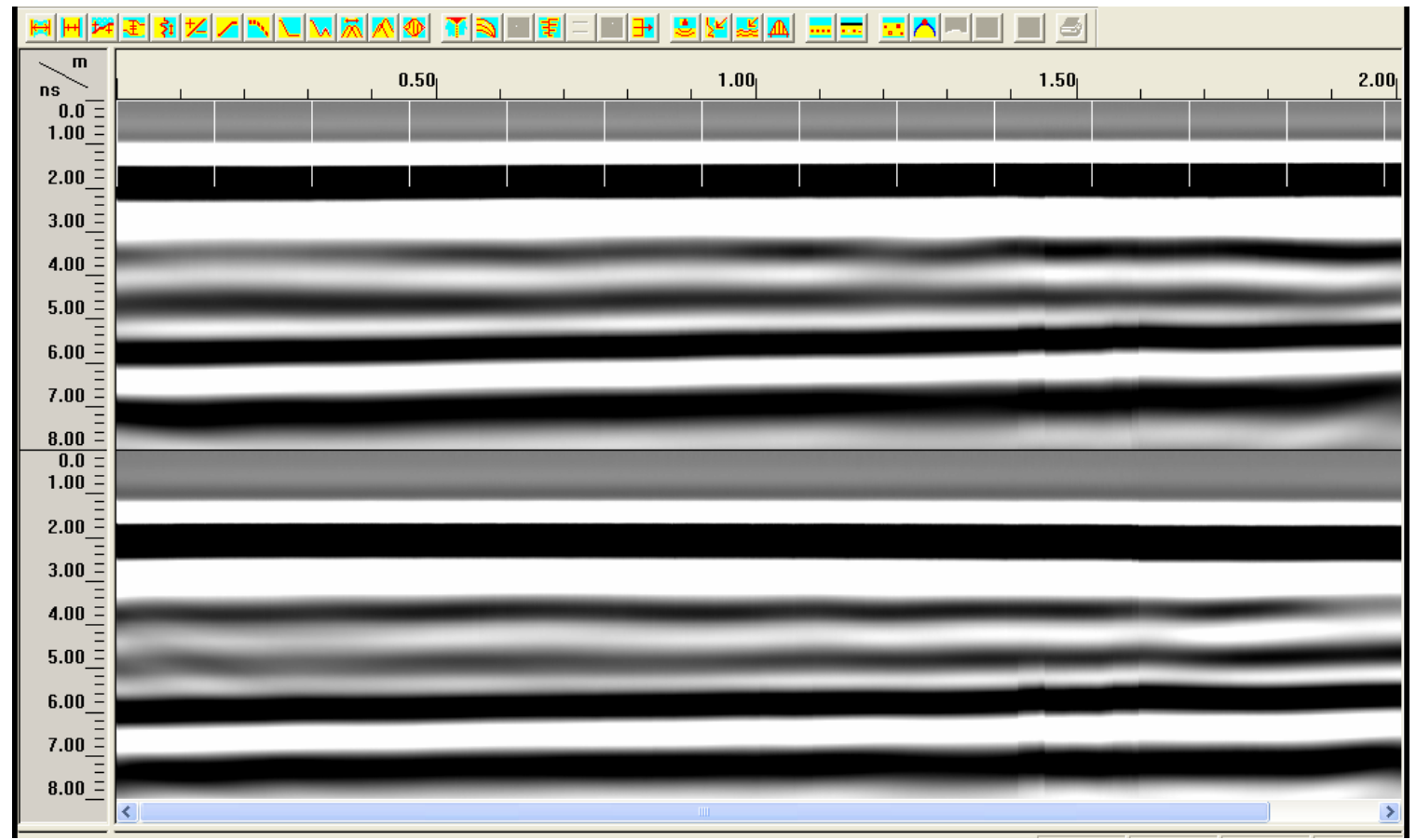

Figure 5-83 Raw GPR data for the antenna configuration shown in Figure 5-82, horizontal scale indicating the travel distance along the length of the $\log (\mathrm{m})$ and vertical scale indicating time (ns) 


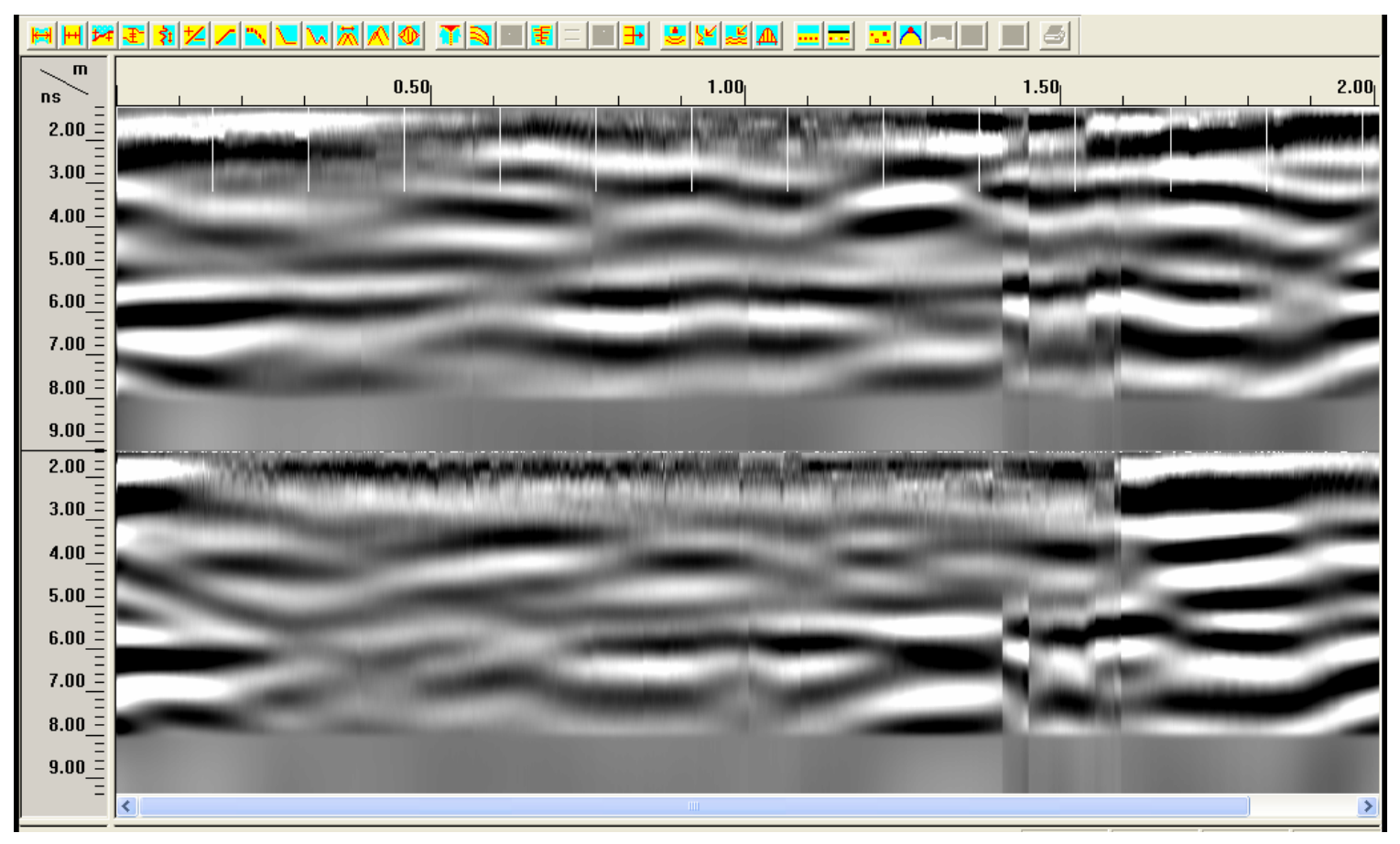

(a)

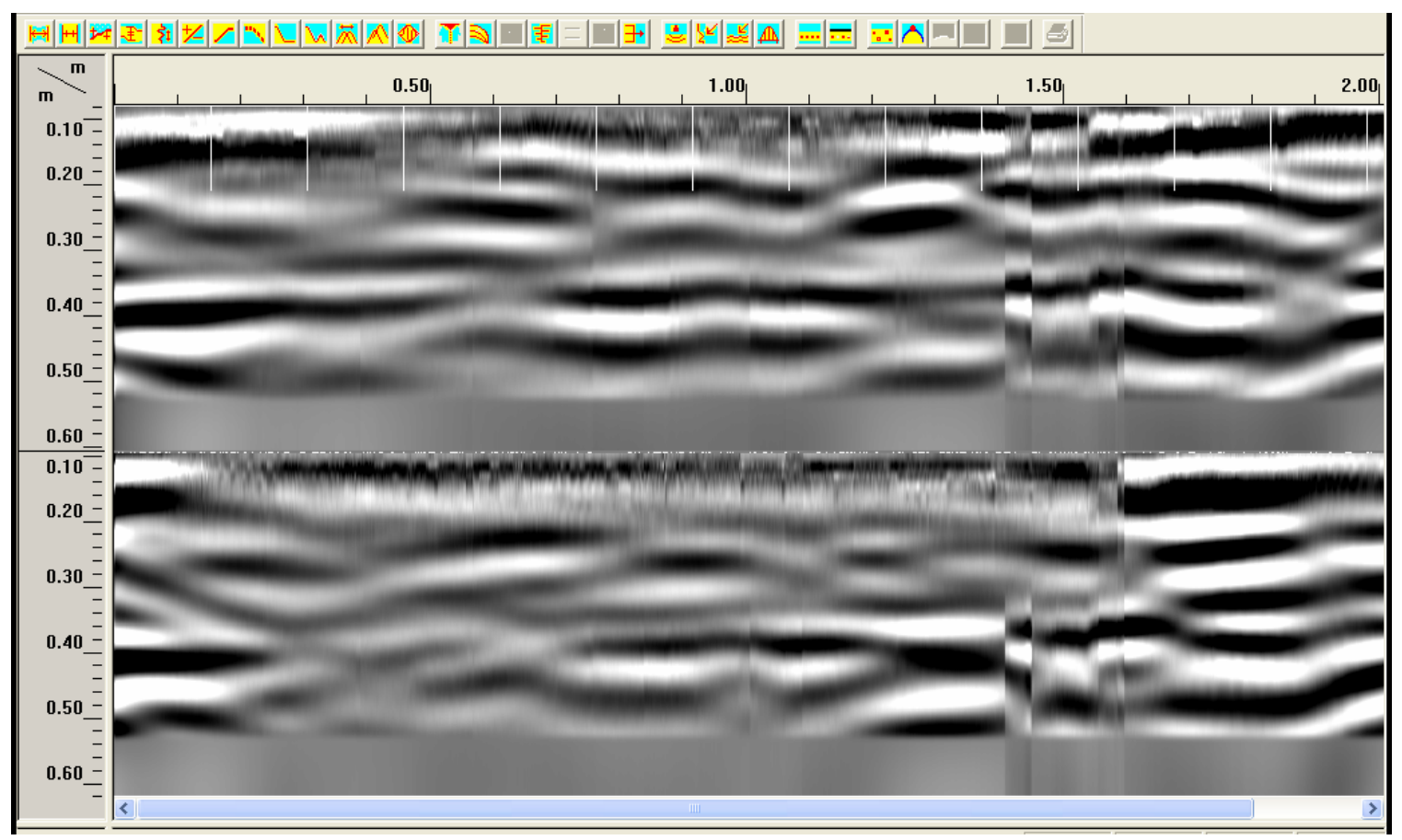

(b)

Figure 5-84 : Processed GPR data for the antenna configuration shown in Figure 5-82, horizontal scale indicating the travel distance along the length of the $\log (\mathrm{m})$ and vertical scale indicating

(a) time (ns) (b) depth (m) 


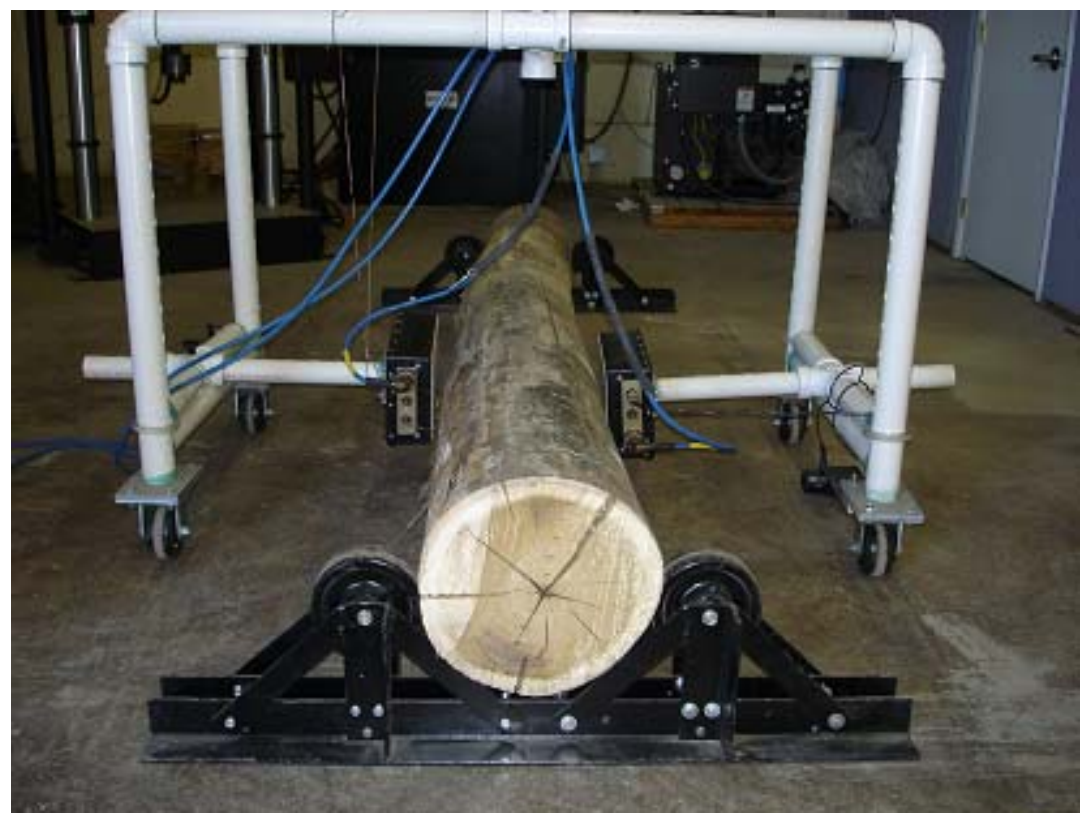

Figure 5-85 Two antennas on the side in T1R2 and T2R1 mode

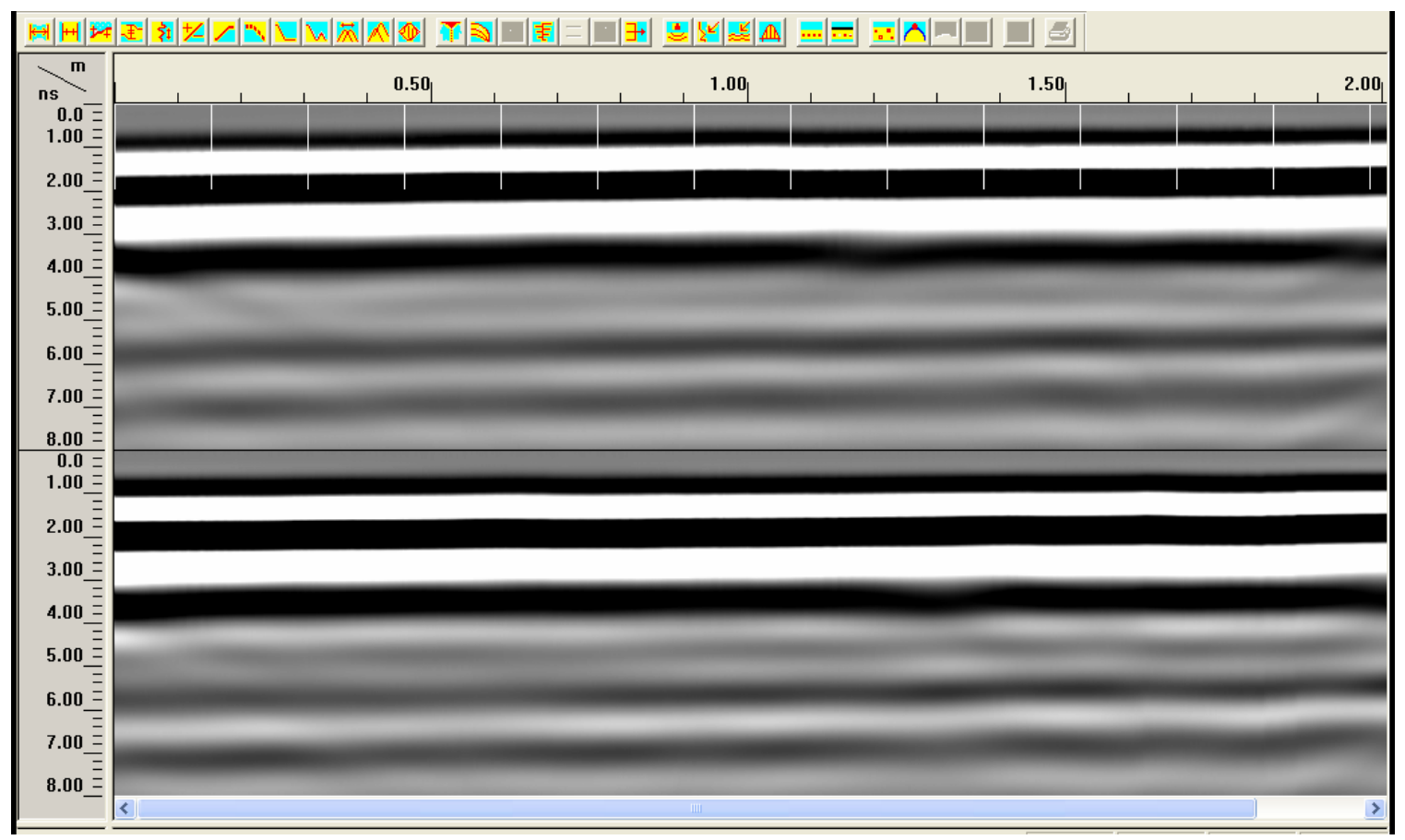

Figure 5-86 Raw GPR data for the antenna configuration shown in Figure 5-85, horizontal scale indicating the travel distance along the length of the $\log (\mathrm{m})$ and vertical scale indicating time (ns) 


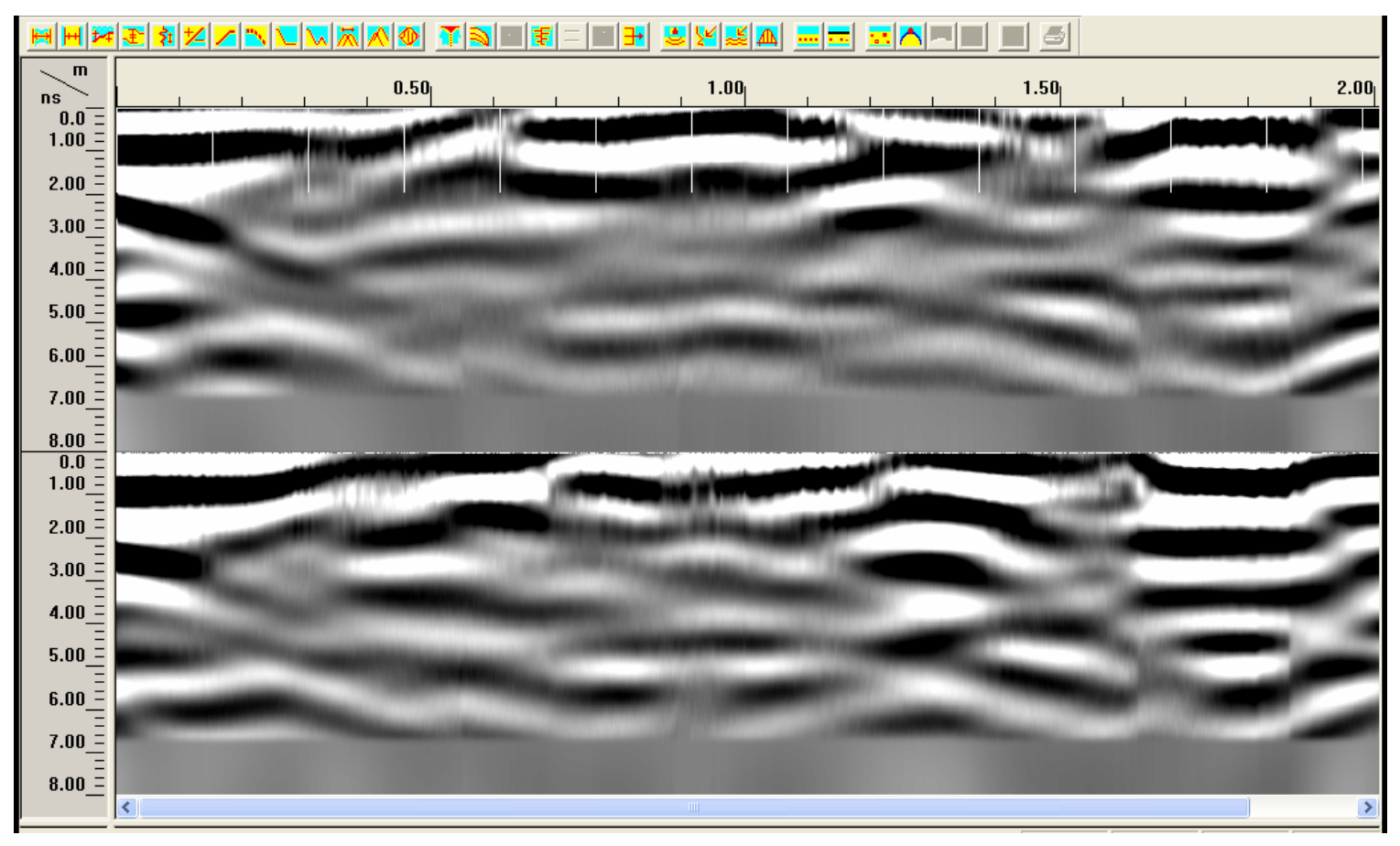

(a)

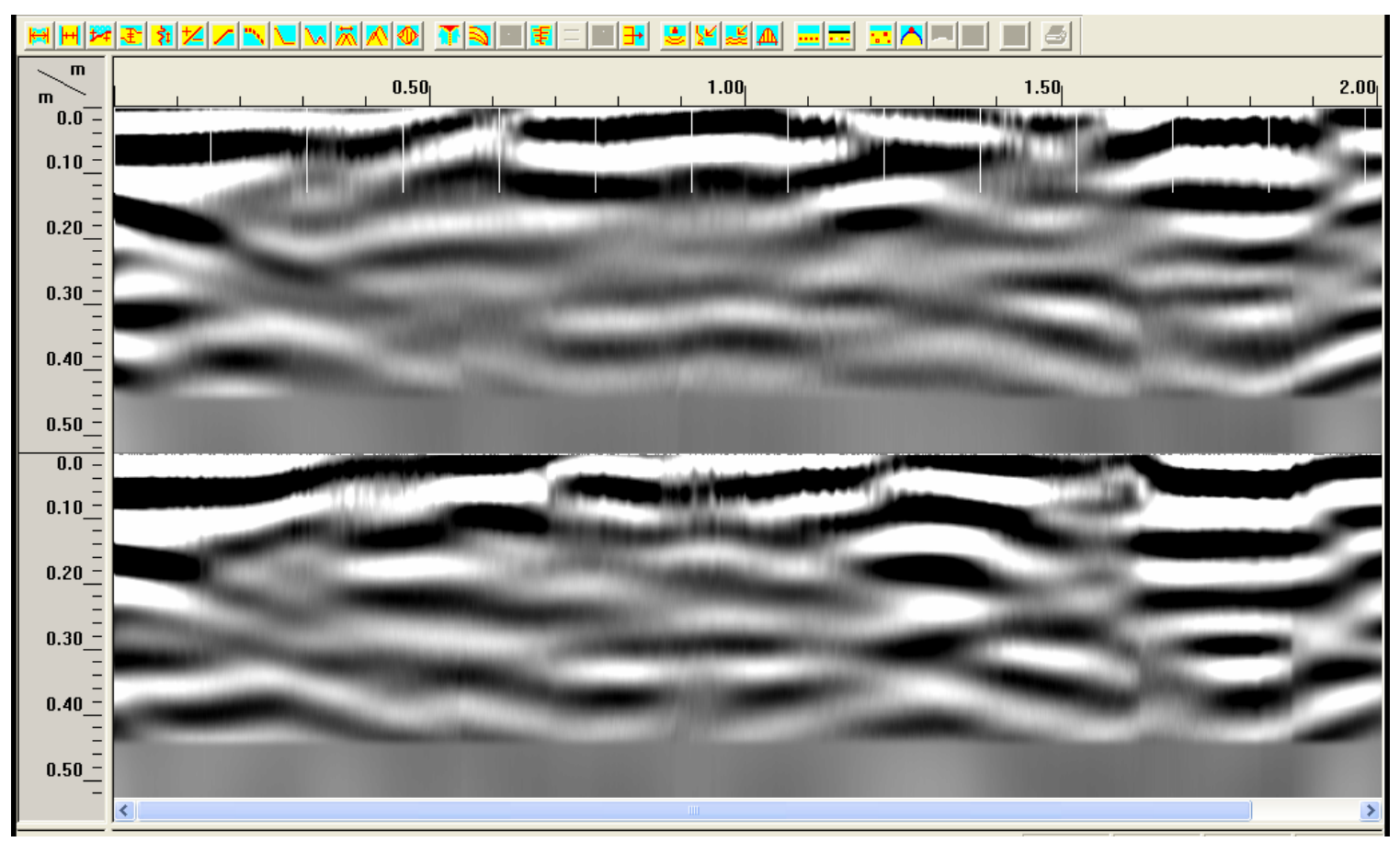

(b)

Figure 5-87 : Processed GPR data of the antenna configuration shown in Figure 5-85, horizontal scale indicating the travel distance along the length of the $\log (\mathrm{m})$ and vertical scale indicating (a) time (ns) (b) depth (m) 


\subsection{SUMMARY}

Data was collected using both single channel and two channel mode. However, for a laboratory type setup where human intervention was the only way of detecting data, single channel mode was preferred over two channel mode. The primary reason for this was that single channel mode provided higher resolution and speedy data collection. Also, data interpretation from the multi-channel mode was somewhat difficult compared to single channel mode.

The disadvantage of using a single channel mode was that it required twice the efforts for data collection and data processing compared to two channel mode. However, the effort in data processing could be reduced by attaching macro in the processing stage. The processing steps can also be included in the data acquisition settings itself for real time processing.

From the above findings it was seen that GPR can detect all types of defects in wooden logs (knots, rots, and embedded metals). It can also detect metal defects of very small size like nails detected in $\log 3$ testing. Detection of small size defects depends on the data collecting parameters and the number of scans per log and how close the scans are (that is, the rotation angle around a log for each new scanning). From all the logs discussed earlier, $\log 3,4$ and 6 have better resolutions compared to other logs because these logs were scanned at a lower scanning rate of $70 \mathrm{scans} / \mathrm{s}$ as opposed to $100 \mathrm{scans} / \mathrm{s}$ for other logs. The location of defects also affects its detection. A defect in the center of the $\log$ is more difficult to detect compared to the defect away from the center due to higher moisture content leading to higher signal attenuation and higher distance to the center (the logs are rotated during scanning). Also, small diameter logs ( $\sim 12$ " or $0.3 \mathrm{~m})$ need only 2 rotations to complete the scanning in order to detect the defects, but may require about 6 to 8 rotations for accurate mapping of the spatial extent of the defects in three dimensions. Such 3-D imaging was outside the scope of the current research. For larger diameter $\operatorname{logs}(\sim 24$ " or $0.6 \mathrm{~m})$ about 16 rotations would be needed for 3-D mapping of the defects.

Tables 5-11 and 5-12 summarize the defects detected in all the logs tested as a part of this study. It can be seen from these tables that knots, rots (decays) and metals 
were all detected, except for a very small sized metal nail in Log 5 (see Table 5-12). Using GPR, the depth resolution of defects was found to be within $0.04 \mathrm{~m}$ and the position along the length of the log could be predicted within $0.02 \mathrm{~m}$.

Table 5-11 Summary of detected defects

\begin{tabular}{|c|c|}
\hline Log & Types of defects located \\
\hline 1 & Knots, Rot Initiation \\
\hline 2 & Knots, Metal \\
\hline 3 & Rot, Metals \\
\hline 4 & Metals \\
\hline 5 & Knots \\
\hline 6 & Rot \\
\hline 7 & Metals \\
\hline
\end{tabular}

Table 5-12 Summary of metal detectability

\begin{tabular}{|c|c|c|c|c|}
\hline \multirow{2}{*}{ Log } & \multirow{2}{*}{ Type of Metal } & \multicolumn{2}{|c|}{ Metal Dimension } & \multirow{2}{*}{ Detectability } \\
\cline { 3 - 5 } & & Diameter (m) & Length (m) & \\
\hline 2 & Rod & 0.016 & 0.1 & Detected \\
\hline 3 & Nail & 0.004 & 0.06 & Detected \\
\hline 4 & Rod & 00095 & 0.08 & Detected \\
\hline 5 & Nail & 0.004 & 0.02 & Not Detected \\
\hline 7 & Bolt & 0.016 & 0.203 & Detected \\
\hline 7 & Rod & 0.019 & 0.2 & Detected \\
\hline
\end{tabular}




\section{Chapter 6 \\ CONCLUSIONS AND RECOMMENDATIONS}

This thesis has presented information about detection and mapping of subsurface defects in logs (knots, decays, and embedded metals) from the GPR scans. Chapter 2 provides information on GPR fundamentals for scanning logs and the equipment setup is given in Chapter 3. Chapter 4 discusses the data collection and basic data processing procedure that was applied to testing of seven logs in order to understand the types of defects that can be detected using GPR. This information was important in understanding the GPR signal characteristics corresponding to the various types of defects and also in selecting the data collection parameters.

Chapter 5 applies the above concepts to the seven logs in order to extract the physical condition of the log from GPR scans. The characteristics of logs, antenna configuration, and data collection parameters applied to the seven logs are given in Chapter 5. Chapter 5 also presents the raw and the processed data which presents a clear view of the subsurface defects. The processed data is then analyzed and interpreted to obtain physical information on the type of defect and its location and spatial extent. Correlation between the extracted defect features after processing and the actual condition seen after sawing is also discussed in Chapter 5.

There was a very good correlation between the predicted defects and those found after sawing the logs. The GPR technique was capable of locating internal defects like knots, decays or rots, and embedded metals. The depth resolution of defects was found to be within $0.04 \mathrm{~m}$ and the position along the length of the log could be predicted within $0.02 \mathrm{~m}$.

This proves that GPR is the most reliable method for locating subsurface defects rapidly and accurately. The system can collect data rapidly and is capable of processing data in real time. The system is suited for online implementation and is also safe, that is, it does not pose any hazard to people around it.

Interpretation of post-processed data of the GPR scan was often complicated and required the use of experienced human personnel. Data was collected using single channel and multi-channel. For a laboratory type setup single antenna was preferred since is resulted in data with better resolution of defects. However, for on-line saw mill 
implementation, it is possible to use multi-channel system with higher resolution by either enhancing the capability of the GPR mainframe or using multiple mainframe systems.

In this research only two values of antenna scanning rate (70 scans/s or 100 scans/s) were explored. Further research has to be conducted in selecting optimum data collection settings and parameters for both single channel and multi-channel modes.

The ability of GPR to detect subsurface defects depends on the direction of the defect with respect to the polarization of the antenna. Defects that are perpendicular to this polarization direction may be completely missed in the GPR scan. Therefore, it is important to explore the use of multiple antenna configurations which include two polarization directions, one along the length of the log and the other perpendicular to it.

Data processing in real time was not conducted in this study. For online implementation of GPR, it is recommended that simultaneous basic data acquisition and processing be carried out and the results compared to the processed data after data collection.

The process of 3D data collection and spatial mapping should be explored to map the size and location of the defect. The current 3-D mapping tool is applicable to planar objects such as bridge decks and pavements but not to radial mapping that is needed for logs. Therefore, algorithm and software for 3D spatial mapping of the defects should also be explored.

All the logs in this study were scanned using GPR after removal of the bark. Further research has to be conducted on logs with and without barks to study the effect of barks on the GPR signals.

Addressing all the above issues is very important for successful development of an automated system for online implementation in saw mills. 


\section{REFERENCES}

Afzal, M. T., Colpits, B., Galik, K. (2003). "Dielectric Properties of Softwood Species Measured with an Open-ended Coaxial Probe." 8th International IUFRO Wood Drying Conference, Brasov, Romania, August 24-29, 110 - 115. (paper also available at http://www.unitbv.ro/il/iufro2003modific/postiufro/contents.htm).

Annan, A. P. (2001). "Ground Penetrating Radar Workshop Notes." Sensors \& Software Inc., Mississauga, Ontario, Canada.

Belli, K. M. (2002). “ Improving the Investigation of Bridge Deck Condition: From Ground Penetrating Radar to a Proposed Defect Assessment Tool." MS Thesis, Department of Civil Engineering, Northeastern University, Boston, MA. (thesis also available at http://www1.coe.neu.edu/ kbelli/thesis/screen-optimized).

Bevan, B. W. (1991). "The Search for Graves." Geophysics, 56(9), 1310-1319.

Canpolar, Inc. (1987). "Preliminary Assessment of Impulse Radar to Detect Decay in Hardwood." Joint Publication of Canadian Forestry Service and the Alberta Forest Service pursuant to the Canada-Alberta Forest Resource Development Agreement, Edmonton, Alberta, Canada.

Detection Sciences, Inc. (1994). "Inspection of Wood with Impulse Radar.” Research Joint Venture Agreement FP-94-2326, USDA Forest Products Laboratory, Madison, WI.

Forest and Wildlife Research Center (2005). http://www.cfr.msstate.edu/fwrc/forestp.htm

GSSI (2005). Geophysical Survey System, Inc., http://www.geophysical.com/

GSSI (2002a). "SIRveyor SIR-20 User's Manual." Geophysical Survey System, Inc., North Salem, NH.

GSSI (2002b). "RADAN for Windows - Version 5.0 User's Manual." Geophysical Survey System, Inc., North Salem, NH.

Green, D. V., Winandy, J. E., Kretschmann, D. E. (1999). "Mechanical Properties of Wood" Chapter 4 of Wood Handbook, U.S. Department of Agriculture, Forest Products Laboratory, Madison, WI. (paper also available at http://www.woodweb.com/knowledge base/Wood Handbook.html).

Gupta, N. K., Schmoldt, D. L., Isaacson, B. (1998). “Tangential Scanning of Hardwood Logs: Developing an Industrial Computer Tomography Scanner." Proceedings of the Eleventh International Symposium on Nondestructive Testing of Wood, Forest Products Society, Madison, WI, Sept. 9-11, 131-139. (paper also available at http://www.srs4702.forprod.vt.edu/pubsubj/pdf/9922.pdf). 
Halabe, U. B., Chen, H. L., Bhandarkar, V., and Sami, Z. (1997). "Detection of SubSurface Anomalies in Concrete Bridge Decks Using Ground Penetrating Radar." American Concrete Institute (ACI) Materials Journal, 94(5), 396-408.

Halabe, U. B., GanagRao H. V. S., Petro, S. H., and Hota, V. R. (1996). “Assessment of Defects and Mechanical Properties of Wood Members Using Ultrasonic Frequency Analysis." Materials Evaluation, 54(2), 314-322.

Halabe, U. B., Petro, S. H., GanagRao H. V. S. (1995). "Nondestructive Evaluation Methods for Highway Bridges Superstructures." CFC 95-215, Constructed Facilities Center, Department of Civil and Environmental Engineering, West Virginia University, Morgantown, WV.

Halabe, U. B., Sotoodehnia, A., Maser, K. R., Kausel, E. A. (1993). "Modeling the Electromagnetic Properties of Concrete." American Concrete Institute (ACI) Materials Journal, 90(6), 552-563.

Keam Holdem Associates Ltd. (1999). "Summary of the Dielectric Permittivity of Wood." Auckland, New Zealand. (paper also available at http://www.kha.co.nz/AP-pdfs/AP0420.pdf).

Loulizi, A. (2001). "Development of Ground Penetrating Radar Signal Modeling and Implementation for transportation Infrastructure Assessment." $\mathrm{PhD}$ Dissertation, Department of Civil Engineering, Virginia Polytechnic Institute and State University, Blacksburg, Virginia. (paper also available at http://scholar.lib.vt.edu/theses/available/etd-02062001-220414/).

Maser, K. R., and Roddis, W. M. K. (1990). "Principles of Thermography and Radar for Bridge Deck Assessment." Journal of Transportation Engineering, ASCE, 116(5), 583-601.

Miller, R. B. (1999a). "Structure of Wood." Chapter 2 of Wood Handbook, U.S. Department of Agriculture, Forest Products Laboratory, Madison, WI. (paper also available at http://www.woodweb.com/knowledge_base/Wood_Handbook.html).

Miller, R. B. (1999b). "Characteristics and Availability of Commercially Important Woods." Chapter 2 of Wood Handbook, U.S. Department of Agriculture, Forest Products Laboratory, Madison, WI. (paper also available at http://www.woodweb.com/knowledge base/Wood Handbook.html).

Muller, W. (2002). "Trial of Ground Penetrating Radar to Locate Defects in Timber Bridge Girders.” Queensland Department of Main Roads, Brisbane, Australia. (paper also available at http://www.ipwea.org.au/papers/download/Muller_W.pdf). 
Ross, R. J., Brashaw, B. K., and Pellerin, R. F. (1998). "Nondestructive Evaluation of Wood.” Forest Products Journal, 48(1), 14-19.

Schad, K. C., Schmoldt, D. L., and Ross, R. J. (1996). "Nondestructive Methods for Detecting Defects in Softwood Logs." Research Paper FPL-RP-546, Forest Products Laboratory, U. S. Department of Agriculture, Madison, WI.

Simpson, W., TenWolde, A. (1999). "Physical Properties and Moisture Relations of Wood." Chapter 3 of Wood Handbook, U.S. Department of Agriculture, Forest Products Laboratory, Madison, WI. (paper also available at http://www.woodweb.com/knowledge_base/Wood_Handbook.html). 